\title{
An Analysis of Evacuation Time Estimates Around 52 Nuclear Power Plant Sites
}

Analysis and Evaluation

Prepared by $\mathrm{T}$. Urbanik II

Texas Transportation Institute

Pacific Northwest Laboratory

Prepared for

U.S. Nuclear Regulatory

Commission 


\section{NOTICE}

This report was prepared as an account of work sponsored by an agency of the United States Government. Neither the United States Government nor any agency thereof, or any of their employees, makes any warranty, expressed or implied, or assumes any legal liability or responsibility for any third party's use, or the results of such use, of any information. apparatus product or process disclosed in this report, or represents that its use by such third party would not infringe privately owned rights.

\section{Available from}

GPO Sales Program

Division of Technical Information and Document Control

U. S. Nuclear Regulatory Comnission

Washington, D. C. 20555

Printed copy price: $\$ 5.50$

and

National Technical Information Service

Springfield, Virginia 22161 
NUREG/CR-1856

PNL-3662

Vol. 1

\section{An Analysis of Evacuation Time Estimates Around 52 Nuclear Power Plant Sites}

Analysis and Evaluation

Manuscript Completed: October 1980

Date Published: May 1981

Prepared by

T. Urbanik II, TTI

A.E. Desrosiers, PNL Project Manager

Texas Transportation Institute

The Texas A\&M University System

College Station, TX 77843

Pacific Northwest Laboratory

Fichland, WA 99352

\section{Prepared for}

Division of Emergency Preparedness

Office of Inspection and Enforcement

U.S. Nuclear Regulatory Commission

Washington, D.C. 20555

NRC FIN No. B2311 

ABSTRACT

On November 29, 1979, the NRC sent a letter to 52 nuclear power plants requesting evacuation time estimates for 10 sectors within a 10-mile radius of each plant. The requirements for these evacuation times are containeo in NUREG-0654, Rev. 1, and include such factors as population density, weather conditions, warning time, response time and confirmation time. Fifty responses were received. The analysis of these findings are presented for review. 

TABLE OF CONTENTS

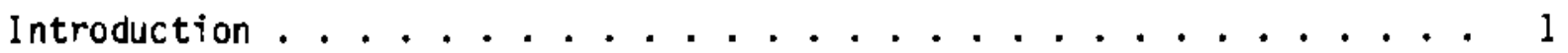
Qualitative Analysis ................. 3 Quantitative Analysis ................. 7

Median Times for 10-Mile Radius .............. 9 Medians by Rating ................ 13 Medians by Total Permanent Population ......... 13 Medians by Sector Permanent Population ......... 15 Median Times by Distance ................ 18

FEMA Assessments . . . . . . . . . . . . . . 23

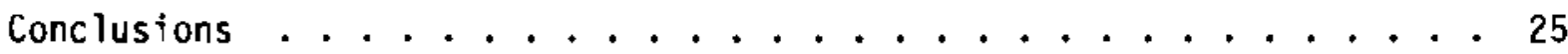
References .................... 27 Appendix A--Evaluation Forms for Individual Plants . . . . . . . A-1 Appendix B--Summary of Evacuation Time Estimates for Individual Plants . B-1 Appendix C--Definitions of Evacuation Time Components ......... C-1 Appendix D--Evaluation Forms for FEMA Assessments ......... . . . Appendix E--Summary of Evacuation Time Estimates for FEMA Assessments . E-1 

LIST OF TABLES

1. Names of Plants Included in the Study .............. 2

2. Evaluation of Evacuation Time Estimate. . . . . . . . . . . . 4 4

3. Results of Subjective Evaluation . . . . . . . . . . . 6

4. Evacuation Time Estimates ................ . . 8

5. Evacuation Time (Hours) Statistics by Component for 10-Mile Radius. . 10

6. Median Evacuation Time (Hours) by Rating for 10-Mile Radius . . . . 14

7. Median Evacuation Time (Hours) by Total Population Groups for 10-Mile Radius. . . . . . . . . . . . . . . 16

8. Median Evacuation Time (Hours) by Sector Permanent Population Groups. 17

9. Median Evacuation Time (Hours) by Sector Permanent Population Groups. 19

10. Median Evacuation Time (Hours) by Distance . . . . . . . . . 21 



\section{INTRODUCTION}

This report presents the findings of evacuation time estimates requested at 52 nuclear power plants by a November 29, 1979 letter from the Nuclear Regulatory Comrassion. The letter from Brian Grimes, Director of Emergency Preparedness Task Group, requested estimates of evacuation times for ten sectors within a radius of about ten miles. Factors to be considered in the analysis included population (permanent, transient and special facilities), weather conditions, warniny time, response time, and confirmation time.

Planning for evacuation as a protective measure is not a new concept in eriergency planning. NUREG-75/111 (1) contained as a planning objective an analysis of estimates of the time required to carry out evacuation procedures. The planning objectives also included the development of evacuation plans for the low population zones (generally about 2 miles). Subsequentiy, NUREG-0396 (2) recommended that NUREG-75/111 should be applied by responsible government officials in larger emergency planning zones

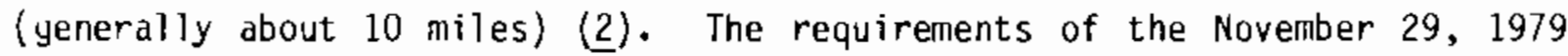
letter referenced above are currently included in NUREG-0654 (3) which is the current interim guidance. These requirements are currently under review and revision.

The 50 responses represented in this analysis include only 49 separate sites as James A. Fitzpatrick and Nine Mile Point plants both occupy the same site. Two plants did not provide estimates. The names of the 52 plants included in this study and the dates of the reports submitted are included in Table 1.

Volume II of this study contains summaries of the evacuation estimates and maps of the plume emergency planning zones around each reactor. 
TABLE 1: NAMES OF PLANTS INCLUDEC IN STUDY

1. Arkansas (1-31-80)

2. Beaver Valley $(1-31-80)$

3. Big Rock Point (6-1-80)

4. Browns Ferry (3-20-80)

5. Brunswick (no response)

6. Calvert Cliffs (1-29-80)

7. Cooper (1-31-80)

8. Crystal River (1-31-80)

9. Davis Besse (8-13-80)

10. D. C. Cook (4-1-80)

11. Diablo Canyon (4-1-80)

12. Dresden $(1-31-80)$

13. Duane Arnold (1-31-80)

14. Farley (2-4-80)

15. Fitzpatrick (1-31-80)

16. Ft. Calhoun $(10-2-80)$

17. Ft. St. Vrain $(2-80)$

18. Ginna $(1-31-80)$

19. Haddam Neck (3-1-80)

20. Hatch $(2-4-80)$

21. Indian Point (1-31-80)

22. Kewaunee (1-29-80)

23. Lacrosse (1-31-80)

24. LaSalle (1-31-80)

25. Maine Yankee (5-23-80)

26. McGuire (1-31-80)
27. Millstone $(3-1-80)$

28. Monticello (4-10-80)

29. Nine Mile Point (1-31-80)

30. North Anna (2-6-80)

31. Oconee (1-31-80)

32. Oyster Creek (1-31-80)

33. Palisades $(6-1-80)$

34. Peach Bottom (1-31-80)

35. Pilgrim (1-31-80)

36. Point Beach (3-26-80)

37. Prairie Island $(4-10-80)$

38. Quad Cities $(1-31-80)$

39. Rancho Seco (1-31-80)

40. Robinson (no response)

41. Saint Lucie (7-17-80)

42. Salem (1-31-80)

43. San Onofre (1-31-80)

44. Sequoyah (3-20-80)

45. Surry (2-6-80)

46. Three Mile 1 sland $(1-31-80)$

47. Trojan (1-31-80)

48. Turkey Point $(7-17-80)$

49. Vermont Yankee $(1-31-80)$

50. Yankee Rowe (1-31-80)

51. Zimmer (8-18-80)

52. Zion $(1-31-80)$

NOTES: Uate of report indicated in parentheses. Fitzpatrick and Nine Mile Point occupy the same site; estimates are only considered once in the analyses. 


\section{QUALITATIVE ANALYSIS}

The first analysis conducted on each evacuation time estimate submittal was a qualitative assessment of the analysis. The standard for comparison was the model plan requirements developed in a separate report entitled "Analysis of Time Required for Evacuation in Emergency Planning Zones Around Nuclear Power Plants" (4).

The evaluation methology used was a four part (excellent, adequate, poor, none) subjective scale using the above mentioned criteria. Each of the items to be discussed subsequently were given a rating as follows. If the criterion was not addressed, the rating given would be none. If the criterion is addressed, but given inadequate consideration, a rating of poor would be assigned. Those submittals providing at least minimum acceptable consideration of the factor would be given an adequate rating. Finally, those analyses that are clearly of high quality and completeness would receive an excellent rating.

Table 2 presents the rating form used which lists the various items considered in the evaluation. The last criterion considered is an overall assessment of the evacuation time estimate. The overall rating is not a simple sumation of the individual factor ratings because the importance of the items considered varies from item to item. Although this analysis requires professional judgement in determining ratings, the process does indicate the area or areas where the reviewer considers the plan to be strong or weak; therefore, a basis exists for resolving weaknesses in plans with poor ratings. Some analyses for which inadequate documentation existed received a poor rating based on the inability to adequately rate the analysis. Table 2 also presents the distribution of ratings by criteria. 
TABLE 2: EVALUATION OF EVACUATION. TIME ESTIMATE

\begin{tabular}{|c|c|c|c|c|}
\hline I tem & Excel. & Adeq. & Poor & None \\
\hline \multicolumn{5}{|l|}{ Background } \\
\hline $\begin{array}{l}\text { A. Area Map } \\
\text { B. Assumpt ions } \\
\text { C. Methodology }\end{array}$ & $\frac{6}{\frac{11}{11}}$ & $\frac{30}{\frac{25}{25}}$ & $\frac{3}{\frac{9}{10}}$ & $\begin{array}{r}11 \\
-\frac{5}{4} \\
\end{array}$ \\
\hline \multicolumn{5}{|l|}{ Demand Estimation } \\
\hline $\begin{array}{l}\text { A. Permanent Population } \\
\text { B. Transient Population } \\
\text { C. Special Population }\end{array}$ & $\frac{11}{\frac{6}{8}}$ & $\frac{29}{\frac{23}{33}}$ & $\frac{\frac{2}{2}}{\frac{2}{3}}$ & $\begin{array}{r}\frac{7}{19} \\
\frac{6}{6} \\
\end{array}$ \\
\hline \multicolumn{5}{|l|}{ Traffic Routing } \\
\hline $\begin{array}{l}\text { A. Map of Network } \\
\text { B. Capacity of Segment }\end{array}$ & $\frac{9}{7}$ & $\frac{25}{22}$ & $\frac{3}{3}$ & $\frac{13}{18}$ \\
\hline \multicolumn{5}{|l|}{ Analysis } \\
\hline $\begin{array}{l}\text { A. Components Considered } \\
\text { B. Adverse Condition Considered }\end{array}$ & $\frac{6}{7}$ & $\frac{34}{25}$ & $\frac{8}{15}$ & $-\frac{2}{3}$ \\
\hline Overa11 & 5 & 28 & 17 & 0 \\
\hline
\end{tabular}

NOTE: The above numbers indicate the number of sites receiving the rating indicated. The two sites not providing estimates are not included. 
Table 3 indicates the overall rating for each of the study sites. Appendix A presents the detailed analyses for each site.

It should be noted that plans receiving an excellent overall rating should not individually be considered as model approaches. Although an excellent response exceeded the average response, in all cases some portions of their approach could be improved upon. Taken as a group, however, the plans rated as excellent represent the state-of-the art in developing evacuation time estimates. 
TABLE 3: RESULTS OF SUBJECTIVE EVALUATION

\begin{tabular}{|c|c|c|c|}
\hline \multicolumn{4}{|c|}{ Overall Rating } \\
\hline Excellent & Adequate & Poor & Wo Response \\
\hline $\begin{array}{l}\text { Diablo Canyon } \\
\text { Indian Point } \\
\text { Pilgrim } \\
\text { Rancho Seco } \\
\text { Trojan }\end{array}$ & $\begin{array}{l}\text { Big Rock Point } \\
\text { Cooper } \\
\text { Crystal River } \\
\text { D. C. Cook } \\
\text { Dresden } \\
\text { Duane Arnold } \\
\text { Fitzpatrick } \\
\text { Ft. St. Vrain } \\
\text { Ginna } \\
\text { Haddan Neck } \\
\text { LaSalle } \\
\text { Maine Yankee } \\
\text { McGuire } \\
\text { Millstone } \\
\text { Monticello } \\
\text { Nine Mile Point } \\
\text { Oyster Creek } \\
\text { Palisades } \\
\text { Peach Bottom } \\
\text { Point Beach } \\
\text { Prairie Island } \\
\text { Quad Cities } \\
\text { St. Lucie } \\
\text { Turkey Point } \\
\text { Vernont Yankee } \\
\text { Yankee Rowe } \\
\text { Zimmer } \\
\text { Zion }\end{array}$ & $\begin{array}{l}\text { Arkansas } \\
\text { Beaver Valley } \\
\text { Browns Ferry } \\
\text { Calvert Cliffs } \\
\text { Davis Besse } \\
\text { Farley } \\
\text { Ft. Calhoun } \\
\text { Hatch } \\
\text { Kewaunee } \\
\text { LaCrosse } \\
\text { North Anna } \\
\text { Oconee } \\
\text { Salem* } \\
\text { San Onofre } \\
\text { Sequoyah } \\
\text { Surry } \\
\text { Three Mile Island }\end{array}$ & $\begin{array}{l}\text { Brunswick } \\
\text { Robinson }\end{array}$ \\
\hline
\end{tabular}

*See footnote on page $A-44$. 
QUANTITATIVE ANALYSIS

The number of plant sites evaluated in the quantitative analys is was 40. Fitzpatrick and Nine Mile Point were only considered once in the analysis. The two plants not providing estimates were not considered in the analysis. Four plants (Browns Ferry, Diablo Canyon, San Onofre and Three Mile Island) were not considered because the responses were not in a format compatible with the analysis. It should be noted that although Diablo Canyon received a an excellent ratiny, it's response was incompatible with the analysis format used. Five sites (Davis Besse, Ft. Calhoun, St. Lucie, Turkey Point, and Zimmer) were not included in the statistical analysis because their responses were received too late.

Table 4 is the form used to sumarize the results found in each evacuation time report submitted. The completeness of data varied greatly anony reports. As will subsequently be seen, some evacuation time components were onitted frequently in the estimates submitted. The individual sumaries are presented in Appendix B.

It must be cautioned that the data submitted was not in the format shown in Table 4. In some cases adjustments or interpretations had to be made to put the data in the format shown. Furthermore, the assumptions underlying the data are not consistent. The following results must be considered as broad indicators of evacuation times at the various plants.

It is appropriate to note at this point that median rather than mean values are used for comparisons in this report. The reason for selecting median values is that in some cases extremely high estimates for a few sites results in a mean value that is not totally representative of the data. 
TABLE 4: EVACUATION TIME ESTIMATES

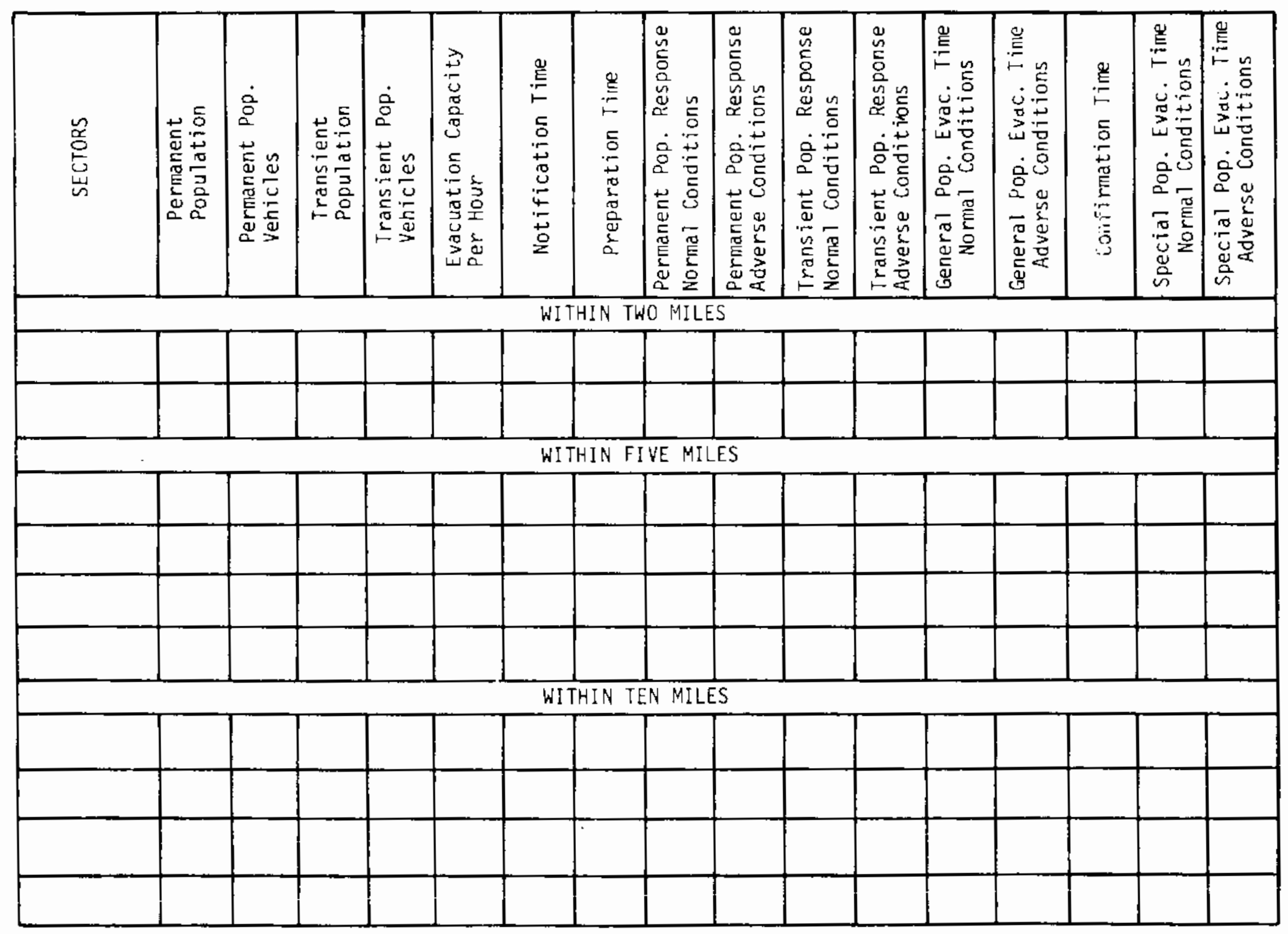


The mean permanent resident population at 40 sites was 58,000 . The median population was 30,000 with the range being 6000 to 282,000 . It should be noted that eight plants used in the analysis did not report population estimates and NUREG 0348 (5) population values for 1970 were used.

The evacuation estimates were requested to be provided for 2-, 5-, and 10-mile radii from the plant. The estimates were also requested for $90^{\circ}$ sectors, except for the 2-mile radius in which $180^{\circ}$ sectors were specified. The remainder of the analysis will center around the 10-mile emergency planning zone. In addition, the effect of distance will be examined by looking at the results by distance.

\section{Median Times for 10-Mile Radius}

The 40 respondents reported results for a total 138 sectors. Not all sites had 4 sectors due to a variety of reasons. Some sites did not report on all sectors due to multistate jurisdictions, some of which did not assist in the assessment. Some sites being adjacent to water bodies do not have 4 sectors on which to estimate land based evacuation. Those plants considering water based evacuations had their over-water evacuation times tallied with special populations so as not to distort land evacuation times.

A total of 109 of the 142 sectors had population data reported. The mean sector population was 17,000. However, the median population was 5000, and 75 percent of the sectors had populations less than 15,000 . The range in sector population was 0 to 151,000 .

Table 5 presents the results of the analysis of time components for the 10-mile radius evacuation. Appendix $C$ defines the terms used. It should also be noted that separate estimates have been made for adverse weather conditions. In most cases, adverse weather conditions resulted in increased 
TABLE 5: EVACUATION TIME (HOURS) STATISTICS BY COMPONENT FOR 10-MILE RADIUS

\begin{tabular}{|l|c|c|c|c|c|c|c|}
\hline $\begin{array}{c}\text { Time } \\
\text { Component* }\end{array}$ & Minimum & $25 \%$ & Median & $75 \%$ & Maximum & \#Sectors & Mean \\
\hline NOTIFY & 0.3 & 0.8 & 1.6 & 3.0 & 6.0 & 116 & 1.9 \\
PPRNC & 0.1 & 1.0 & 1.8 & 4.0 & 8.3 & 108 & 2.7 \\
PPRAC & 0.2 & 1.3 & 2.8 & 6.0 & 16.2 & 100 & 4.1 \\
TPRNC & 0.4 & 1.3 & 3.0 & 3.5 & 3.7 & 11 & 2.4 \\
TPRAC & 0.6 & 0.8 & 1.7 & 4.1 & 4.2 & 7 & 2.4 \\
GP TNC & 1.0 & 2.9 & 5.0 & 8.0 & 21.0 & 111 & 5.3 \\
GPTAC & 1.3 & 3.0 & 5.2 & 8.8 & 18.3 & 91 & 6.4 \\
CONFIRM & 0.6 & 1.0 & 2.0 & 4.0 & 24.0 & 75 & 3.5 \\
SPRNC & 0.3 & 1.3 & 2.7 & 4.9 & 24.0 & 44 & 3.7 \\
SPRAC & 0.6 & 2.4 & 3.2 & 8.0 & 10.2 & 28 & 4.7 \\
\hline
\end{tabular}

\footnotetext{
*NOTIFY $=$ NOTIFICATION TIME

PPRNC = PERMANENT POPULATION RESPONSE TIME NORMAL CONDITIONS

PPRAC $=$ PERMANENT POPULATION RESPONSE TIME ADVERSE CONDITIONS

TPRNC $=$ IRANSIENT POPULATION RESPONSE TIME NORMAL CONDITIONS

TPRAC = TRANSIENT POPULATION RESPONSE TIME AUVERSE CONDITIONS

GPTNC = GENERAL POPULATION EVACUATION TIME NORMAL CONDITIONS

GPTAC = GENERAL POPULATION EVACUATION TIME ADVERSE CONDITIONS

CONFIRM $=$ CONFIRMATION TIME

SPRNC = SPECIAL POPULATION RESPONSE TIME NORMAL CONUITIONS

SPRAC = SPECIAL POPULATION RESPONSE TIME ADVERSE CONDITIONS

SEE APPENDIX C FOR DEFINITION.
} 
response time. However, at some sites, the adverse weather alternative was assumed to occur only in the winter when transient populations would be low or nonexistent. Therefore, in some cases, adverse weather response time was estimated to be less than for normal conditions when large transient populations would be possible. Although this approach is not recommended, it nevertheless was the approach used by some. The proper approach would have been to consider other adverse conditions (e.g., rain) under peak demand.

Estimates for total notification time varied from 0.3 hours to a maximum of 6.0 hours. A few of the studies looked at the interaction between warning time and response time. A few studies estimated the distribution for warning and preparation in determining response times. Others added total warning time plus response time and indicated that the result overestimated the total time required because some people would begin evacuating before others are warned.

Further study is warranted in examining the interaction between components because of the uncertainty concerning the effect on total evacuation time. Improved notification systems may pose problems at some sites since they could induce traffic volumes that exceed the capacity of the roadway system. Thus, even though notification times were greatly reduced, evacuation times might be largely unaffected or even increase. Such situations would require careful traffic management in order to avoid massive traffic jams. One satisfactory solution for these sites might be immediate (15 minute) notification followed by phased evacuation in combination with an effective sheltering program.

The median response time for permanent residents was 1.8 hours for normal conditions and 2.8 hours for adverse conditions (see PPRNC and PPRAC 
in Table 5). The upper limits were 8.3 and 16.2 hours for normal and adverse conditions respectively.

The median transient population response time was 3.0 hours for normal conditions and 1.7 hours for adverse conditions (see TPRNC and TPRAC in Table 5). Transient population response time was a time component in only 11 sectors under normal conditions and 7 sectors under adverse conditions. Maximum values were estimated at 3.7 hours under normal conditions and 4.2 under adverse conditions. As previously indicated, some estimates for adverse conditions were less because transient populations were expected to be smaller under adverse conditions which was assumed to be snow. 0ther adverse conditions such as rain were not considered.

The median special population response time was 2.7 hours (44 sectors) for normal conditions and 3.2 hours (28 sectors) for adverse conditions. Maximum values were 24 hours for normal conditions and 10.2 hours for adverse conditions. The lower maximum value for adverse conditions results from an adverse weather estimate not being made for the site reporting the maximum normal weather time.

As previously indicated, notification plus response time estimates were summed to estimate the time required (not including confirmation) to clear the sector after issuance of a warning. As previously indicated, most estimates include a simple summation of notification and response time which may overestimate the required time since some people will leave before all are notified. The median general population evacuation time was 5.0 hours (111 sectors) under normal conditions and 5.2 hours (91 sectors) under adverse conditions. Maximum times were 21.0 hours for normal conditions and 27.0 hours for adverse conditions. 
Confirmation times ranged from 0.6 hours to 24.0 hours, with the median being 2.0 hours. Confirmation time estimates were based on many different assumptions. The effectiveness of confirmation seened to be questioned by many. The accuracy of the estinates seems questionable because of the lack of yood data on which to base an estimate.

Medians by Rating

In an earlier section of the report a subjective rating was made and each of the responses were rated poor, adequate, or excellent. In order to determine if the results differed by rating, the medians were separately calculated for each rating class. Fourteen sites (51 sectors) had a poor ratiny, 22 sites (73 sectors) had an adequate rating, and 4 sites (15 sectors) had an excellent rating. Table 6 summarizes the evacuation time means by rating for the various time components. The lower ratings appear to have generally higher estimates for general population evacuation times. However, subsequent analysis using the data segregated by rating did not appear to improve the results observed. The remaining analyses include all the data including responses receiving a poor rating.

\section{Medians by Total Permanent Population}

As indicated earlier, the population distribution while varying from 6000 to 282,000 , has a median of only 30,000 . A second stratification of the data, based on total perfianent resident population, was therefore made. Five categories were established as follows:

$$
\begin{array}{lr}
\text { 1. } & 1-20,000 \\
\text { 2. } & 20,000-50,000 \\
\text { 3. } & 50,000-100,000
\end{array}
$$


TABLE 6: MEUIAN EVACUATION TIME (HOURS) BY RATING FOR 10-MILE RADIUS

\begin{tabular}{|c|c|c|c|c|c|c|c|c|}
\hline \multirow[b]{3}{*}{ Component* } & \multicolumn{8}{|c|}{ Rating } \\
\hline & \multicolumn{2}{|c|}{ Poor } & \multicolumn{2}{|c|}{ Adequate } & \multicolumn{2}{|c|}{ Excellent } & \multicolumn{2}{|c|}{ Overall } \\
\hline & Median & $\begin{array}{l}\# \text { of } \\
\text { Sectors }\end{array}$ & Median & $\begin{array}{c}\text { of } \\
\text { Sectors }\end{array}$ & Median & $\begin{array}{c}\# \text { of } \\
\text { Sectors }\end{array}$ & Median & $\begin{array}{c}\text { of } \\
\text { Sectors }\end{array}$ \\
\hline NOTIFY & 2.0 & 42 & 1.8 & 59 & 1.5 & 15 & 1.6 & 116 \\
\hline PPRNC & 3.0 & 38 & 1.5 & 55 & 1.4 & 15 & 1.8 & 108 \\
\hline PPRAC & 5.0 & 38 & 2.0 & 47 & 2.1 & 15 & 2.8 & 100 \\
\hline TPRNC & 3.0 & 7 & 2.3 & 1 & 3.1 & 3 & 3.0 & 11 \\
\hline TPRAC & 2.7 & 6 & 0.8 & 1 & -- & -- & 1.7 & 7 \\
\hline GPTNC & 6.0 & 40 & 5.0 & 56 & 4.7 & 15 & 5.0 & 111 \\
\hline $\begin{array}{l}\text { GPTAC } \\
\text { CONFIRM } \\
\text { SPRNC } \\
\text { SPRAC }\end{array}$ & $\begin{array}{l}7.3 \\
2.0 \\
2.7 \\
3.2\end{array}$ & $\begin{array}{r}39 \\
32 \\
10 \\
9\end{array}$ & $\begin{array}{l}4.9 \\
2.0 \\
1.9 \\
2.9\end{array}$ & $\begin{array}{l}37 \\
29 \\
29 \\
14\end{array}$ & $\begin{array}{r}4.4 \\
2.0 \\
7.2 \\
10.2\end{array}$ & $\begin{array}{r}15 \\
14 \\
5 \\
5\end{array}$ & $\begin{array}{l}5.2 \\
2.0 \\
2.7 \\
3.2\end{array}$ & $\begin{array}{l}91 \\
75 \\
44 \\
28\end{array}$ \\
\hline
\end{tabular}

ॠNOTIFY = NOTIFICATION TIME

PPRNC = PERMANENT POPULATION RESPONSE TIME NORMAL CONOITIONS

PPRAC $=$ PERMANENT POPULATION RESPONSE TIME AOVERSE CONDITIONS

TPRNC $\Rightarrow$ TRANSIENT POPULATION RESPONSE TIME NORMAL CONDITIONS

TPRAC = TRANSIENT POPULATION RESPONSE TIME AUVERSE CONOITIONS

GPTNC = GENERAL POPULATION EVACUATION TIME NORMAL CONOITIONS

GPTAC = GENERAL PUPULATION EVACUATION TIME ADVERSE CONDITIONS

CONFIRM $=$ CONFIRMATION TIME

SPRNC = SPECIAL PDPULATION RESPONSE TIME NORMAL CONDITIONS

SPRAC = SPECIAL POPULATION RESPONSE TIME AUVERSE CONDITIONS

SEE APPENDIX C FOR DEFINITIONS. 

4. $100,000-200,000$
5. 200,000 and up

There were 11 sites in category 1,15 sites in category 2,8 sites in category 3,3 sites in category 4 , and 3 sites in category 5 .

Table 7 sumarizes the evacuation time estimates for the five population groups. No clear trends appear in the data. Several other analyses were performed including deletion of data receiving a poor rating and use of mean instead of median values. These additional analyses also did not reveal any consistent trends.

Because the population density could vary by sector, and since no trends were found based on total population, it was determined that evacuation time components should be evaluated based on sector population. The following section examines the data based on sector population.

\section{Medians by Sector Permanent Population}

The sector populations were broken into groups for analys is as follows:

$$
\begin{array}{lr}
\text { 1. } & 1-5,000 \\
\text { 2. } & 5,000-10,000 \\
\text { 3. } & 10,000-25,000 \\
\text { 4. } & 25,000-50,000 \\
\text { 5. } & 50,000-100,000 \\
\text { 6. } & 100,000 \text { and up }
\end{array}
$$

Not all sites reported sector population so that the number of sectors analyzed is less than for the total population groups.

Table 8 summarizes the medians by sector population. 0ne trend noted in the data is that permanent population response time (normal and adverse) 
TABLE 7: MEDIAN EVACUATION TIME (HOURS) BY TOTAL POPULATION GROUPS FOR 10-MILE RADIUS

\begin{tabular}{|c|c|c|c|c|c|c|c|c|c|c|c|c|}
\hline \multirow[b]{2}{*}{ Component* } & \multicolumn{2}{|c|}{$\begin{array}{c}\text { Total Popu1ation } \\
1-20,000\end{array}$} & \multicolumn{2}{|c|}{$\begin{array}{l}\text { Total Population } \\
20,000-50,000\end{array}$} & \multicolumn{2}{|c|}{$\begin{array}{l}\text { Total Population } \\
50,000-100,000\end{array}$} & \multicolumn{2}{|c|}{$\begin{array}{l}\text { Total Population } \\
100,000-200,000\end{array}$} & \multicolumn{2}{|c|}{$\begin{array}{l}\text { Total Population } \\
200,000 \text { and up }\end{array}$} & \multicolumn{2}{|c|}{ Overail } \\
\hline & Median & $\begin{array}{l}\# \text { of } \\
\text { Sectors }\end{array}$ & Median & $\begin{array}{l}\# \text { of } \\
\text { Sectors }\end{array}$ & Median & $\begin{array}{l}\# \text { of } \\
\text { Sectors }\end{array}$ & Median & $\begin{array}{l}\# \text { of } \\
\text { Sectors }\end{array}$ & Median & $\begin{array}{l}\# \text { of } \\
\text { Sectors }\end{array}$ & Median & $\begin{array}{l}\# \text { of } \\
\text { Sectors }\end{array}$ \\
\hline NOTIFY & 3.0 & 29 & 1.1 & 46 & 2.0 & 29 & 2.6 & 6 & 1.4 & 6 & 1.6 & 116 \\
\hline PPRNC & 3.0 & 35 & 3.0 & 42 & 1.3 & 21 & 1.9 & 6 & 3.5 & 4 & 1.8 & 108 \\
\hline PPRAC & 4.3 & 23 & 2.0 & 46 & 1.7 & 21 & 2.8 & 6 & 5.6 & 4 & 2.8 & 100 \\
\hline TPRNC & 3.5 & 1 & 2.3 & 1 & 1.5 & 7 & 3.4 & 2 & --- & - & 3.0 & 11 \\
\hline TPRAC &.-- & - & 0.8 & 1 & 1.6 & 4 & 4.1 & 2 & -- & - & 1.7 & 7 \\
\hline GPTNC & 4.0 & 30 & 3.7 & 38 & 5.7 & 29 & 5.8 & 7 & 5.0 & 6 & 5.0 & 111 \\
\hline GPTAC & 4.4 & 17 & 3.8 & 37 & 5.1 & 25 & 7.3 & 7 & 6.0 & 4 & 5.2 & 91 \\
\hline CONFIRM & 4.4 & 16 & 2.0 & 31 & 1.0 & 21 & 2.0 & 4 & 2.0 & 3 & 2.0 & 75 \\
\hline SPRNC & 0.8 & 10 & 2.4 & 20 & 4.0 & 7 & 2.8 & 1 & 7.2 & 6 & 2.7 & 44 \\
\hline SPRAC & 3.0 & 5 & 3.2 & 13 & 5.0 & 5 & 2.8 & 1 & 10.2 & 4 & 3.2 & 28 \\
\hline
\end{tabular}

-NOTIFY = NOTIFICATION TIME

PPRNC = PERMANENT POPULATION RESPONSE TIME NORMAL CONOITIONS

PPRAC = PERMANENT POPULATION RESPONSE TIME ADVERSE CONDITIONS

TPRNC = TRANSIENT POPULATION RESPONSE TIME NORMAL CONOITIONS

TPRAC = TRANSIENT POPULATION RESPONSE TIME ADYERSE CONDITIONS

GPTNC = GENERAL POPULATION EYACUATION TIME NORMAL CONOITIONS

GPTAC = GENERAL POPULATION EVACUATION TIME ADVERSE CONDITIONS

CONFIRM = CONFIRMATION TIME

SPRNC = SPECIAL POPULATION RESPONSE TIME NORMAL CONOITIONS

SPRAC = SPECIAL POPULATION RESPONSE TIME ADYERSE CONDITIONS

SEE APPENDIX C FOR OEFINITIONS. 
TABLE 8: MEDIAN EVACUATION TIME (HOURS) BY SECTOR PERMANENT POPULATION GROUPS

\begin{tabular}{|c|c|c|c|c|c|c|c|c|c|c|c|c|}
\hline \multirow[b]{2}{*}{ Component* } & \multicolumn{2}{|c|}{$\begin{array}{c}\text { Sector } \\
\text { Population } \\
0-5,000\end{array}$} & \multicolumn{2}{|c|}{$\begin{array}{c}\text { Sector } \\
\text { Population } \\
5,000-10,000\end{array}$} & \multicolumn{2}{|c|}{$\begin{array}{c}\text { Sector } \\
\text { Population } \\
10,000-25,000\end{array}$} & \multicolumn{2}{|c|}{$\begin{array}{c}\text { Sector } \\
\text { Population } \\
25,000-50,000\end{array}$} & \multicolumn{2}{|c|}{$\begin{array}{c}\text { Sector } \\
\text { Population } \\
50,000-100,000\end{array}$} & \multicolumn{2}{|c|}{$\begin{array}{c}\text { Sector } \\
\text { population } \\
100,000 \text { and up }\end{array}$} \\
\hline & Median & $\begin{array}{l}\# \text { of } \\
\text { Sectors }\end{array}$ & Median & $\begin{array}{l}\# \text { of } \\
\text { Sectors }\end{array}$ & Median & $\begin{array}{l}\# \text { of } \\
\text { Sectors }\end{array}$ & Median & $\begin{array}{l}\# \text { of } \\
\text { Sectors }\end{array}$ & Median & $\begin{array}{l}\# \text { of } \\
\text { Sectors }\end{array}$ & Median & $\begin{array}{l}\# \text { of } \\
\text { Sectors }\end{array}$ \\
\hline NOTIFY & 2.0 & 44 & 1.0 & 18 & 1.1 & 17 & 2.0 & 9 & 1.7 & 2 & 1.4 & 6 \\
\hline PPRNC & 1.4 & 43 & 1.5 & 14 & 1.4 & 13 & 3.5 & 8 & 4.2 & 2 & 3.7 & 4 \\
\hline PPRAC & 1.5 & 38 & 2.0 & 12 & 1.9 & 14 & 5.2 & 8 & 4.8 & 2 & 5.8 & 4 \\
\hline TPRNC & 3.5 & 1 & 2.5 & 2 & 3.0 & 5 & 1.3 & 2 & 3.4 & 1 & $-\infty$ & - \\
\hline TPRAC & --- & -- & 1.7 & 1 & 2.3 & 4 & 1.5 & 1 & 4.1 & 1 & --- & - \\
\hline GPTNC & 3.9 & 42 & 6.5 & 17 & 4.3 & 14 & 5.3 & 8 & 7.6 & 2 & 6.6 & 6 \\
\hline GPTAC & 4.4 & 34 & 4.5 & 11 & 4.5 & 13 & 5.7 & 7 & 8.5 & 2 & 7.1 & 4 \\
\hline CONFIRM & 2.2 & 31 & 2.0 & 10 & 1.0 & i1 & 3.5 & 7 & 2.0 & 1 & 2.0 & 3 \\
\hline SPRNC & 0.8 & 8 & 1.9 & 10 & 2.7 & 7 & 4.0 & 5 & 3.7 & 1 & 7.2 & 6 \\
\hline SPRAC & 2.0 & 4 & 3.1 & 4 & 3.1 & 4 & 5.0 & 4 & 4.7 & 1 & 10.2 & 4 \\
\hline
\end{tabular}

*NOTIFY = NOTIFICATION TIME

PPRNC = PERMANENT POPULATION RESPONSE TIME NORMAL CONDITIONS

PPRAC = PERMANENT POPULATION RESPONSE TIME ADVERSE CONDITIONS

TPRNC = TRANSIENT POPLLATION RESPONSE TIME NORMAL CONOITIONS

TPRAC = TRANSIENT POPULATION RESPONSE TIME ADVERSE CONDITIONS

GPTNC = GENERAL POPULATION EVACUATION TIME NORIAAL CONUITIONS

GPTAC = GENERAL POPULATION EVACUATION TIME AOVERSE CONUITIONS

CONFIRM $=$ CONFIRMATION TIME

SPRNC = SPECIAL POPULATION RESPONSE TIME NORMAL CONDITIONS

SPRAC = SPECIAL POPULATION RESPONSE TIME ADVERSE CONDITIONS

SEE APPENOIX C FOR DEFINITIONS. 
appears yreater for sectors above 25,000 than below 25,000. Another trend is that special population response time (normal and adverse) appears to increase with increasing sector populations. In order to further evaluate these trends, the data was combined into two groups. Table 9 summarizes the medians with only two sector population yroups: above 25,000 and below 25,000. The trend toward increased permanent population response time appears fairly strong. Figure 1 shows the actual distribution of responses for the two population groups. The under 25,000 group shows a long tail to the right distribution typical of the data in general. The over 25,000 population group shows an extremely strong tendency toward its median (and therefore mean value due to the symetrical distribution) value.

It should also be noted in Table 9 that the smaller difference between the GPTNC for the two groups may be explained in part by a higher transient population response time for the under 25,000 population group. The special population response times also continue to show a much larger value for the over 25,000 population group.

\section{Median Times by Distance}

Table 10 sumarizes the median evacuation time components for the 2-, 5-, and 10-mile radii from the plants. As would be expected, the times increase with increasing distance. What is surprising is that permanent population response time increase is less than proportional to the distance and that general population evacuation time increase is nearly proportional to distance for the increase in area from five to ten miles. Further study of the effect of distance is warranted. 
TABLE 9: MEOIAN EVACUATION TIME (HOURS) BY SECTOR PERMANENT PUPULATION GROUPS

\begin{tabular}{|l|c|c|c|c|}
\hline & \multicolumn{2}{|c|}{$\begin{array}{c}\text { Sector } \\
\text { Population } \\
0-25,000\end{array}$} & \multicolumn{2}{c|}{$\begin{array}{c}\text { Sector } \\
\text { Population } \\
25,000 \text { and up }\end{array}$} \\
\cline { 2 - 5 } Component* & Median & $\begin{array}{c}\text { Sectors } \\
\text { Sectory }\end{array}$ & Median & $\begin{array}{c}\text { Sectors } \\
\text { Sectors }\end{array}$ \\
\hline NOTIFY & 1.3 & 79 & 1.5 & 17 \\
PPRNC & 1.4 & 70 & 3.7 & 14 \\
PPRAC & 1.9 & 64 & 5.5 & 14 \\
TPRNC & 3.1 & 8 & 1.5 & 3 \\
TPRAC & 1.7 & 5 & 2.8 & 2 \\
GPTNC & 5.0 & 73 & 5.7 & 16 \\
GPTAC & 4.5 & 58 & 6.7 & 13 \\
CUNFIRM & 2.0 & 52 & 2.0 & 11 \\
SPRNC & 1.9 & 25 & 6.1 & 12 \\
SPRAC & 3.1 & 12 & 5.0 & 9 \\
\hline
\end{tabular}

\footnotetext{
*NOTIFY $=$ NOTIFICATION TIME

PPRNC = PERMANENT POPULATION RESPONSE TIME NORMAL CONDITIONS

PPRAC = PERMANENT POPULATION RESPONSE TIME ADVERSE CUNDITIONS

TPRNC = TRANSIENT POPULATION RESPONSE TIME NORMAL CONDITIONS

TPRAC = TRANSIENT POPULATION RESPONSE TIME ADVERSE CONDITIONS

GPTNC = GENERAL POPULATION EVACUATION TIME NORMAL CONUITIONS

GPTAC = GENERAL POPULATION EVACUATION TIME ADVERSE CONDITIDNS

CONF IRM = CONF IRMATION TIME

SPRNC = SPECIAL POPULATION RESPONSE TIME NORMAL CONDITIONS

SPRAC = SPECIAL POPULATION RESPONSE TIME ADVERSE CONDITIONS
}

SEE APPENDIX C FOR DEFINITIONS. 


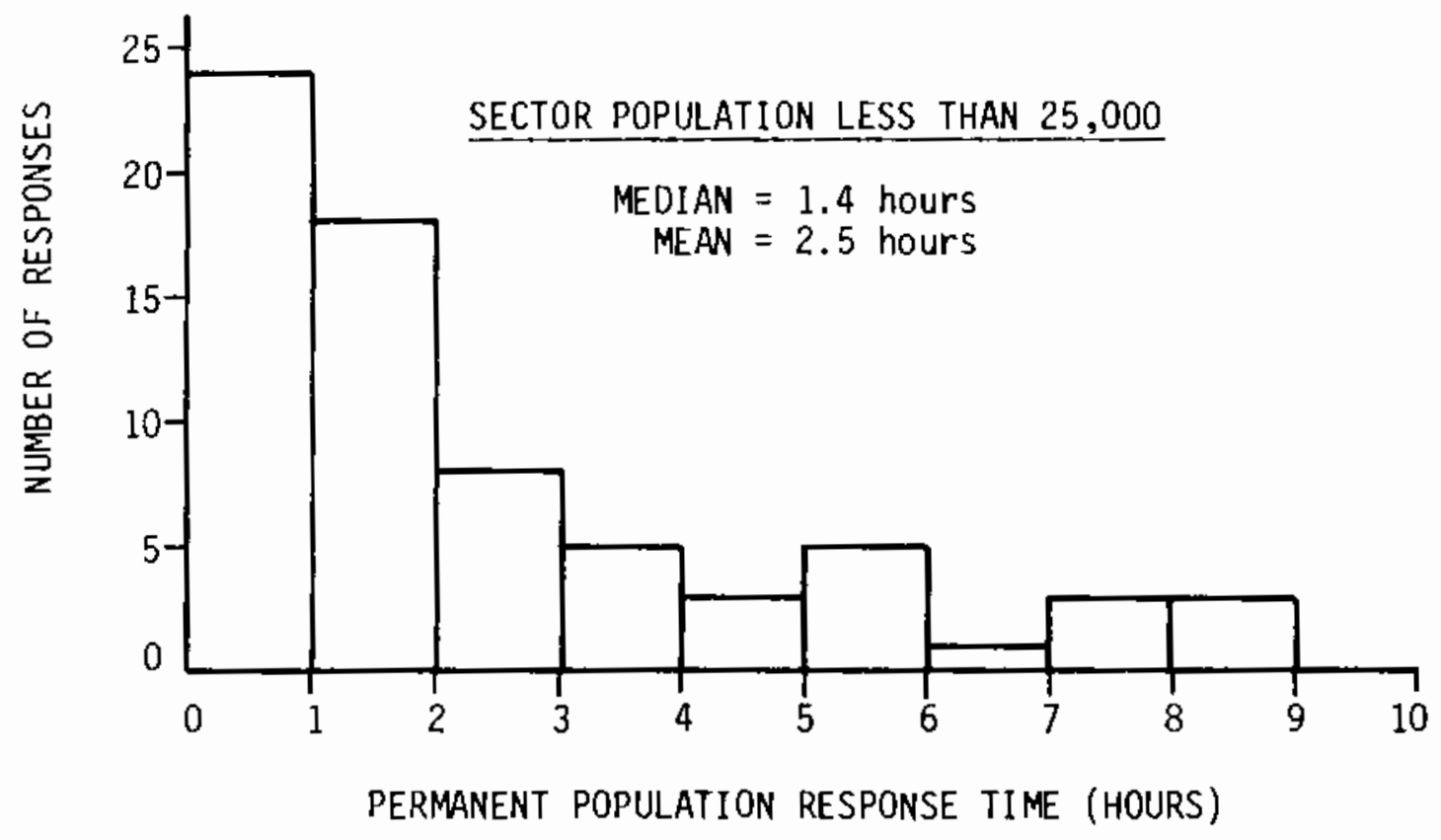

SECTOR POPULATION GREATER THAN 25,000

MEDIAN $=3.7$ hours

MEAN $=3.7$ hours

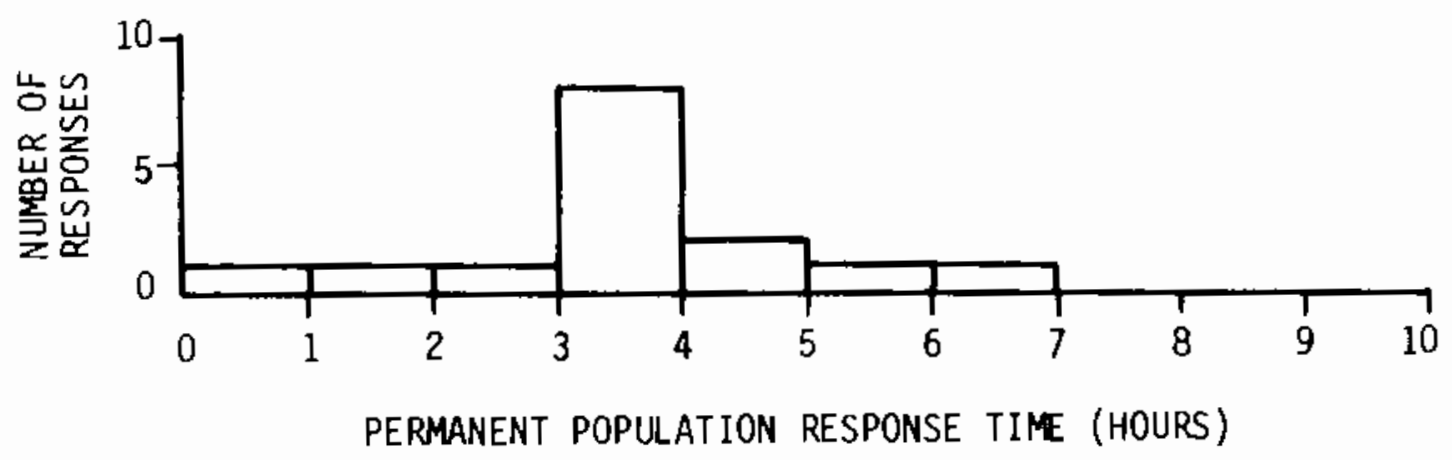

FIGURE 1: PERMANENT POPULATION RESPONSE TIME BY SECTOR POPULATION GROUP 
TABLE 10: MEDIAN EVACUATION TIME (HOURS) BY DISTANCE

\begin{tabular}{|l|c|l|c|c|c|c|}
\hline \multirow{2}{*}{ Coriponent* } & \multicolumn{2}{|c|}{ Radius $=2 \mathrm{mi}}$. & \multicolumn{2}{c|}{ Radius $=5 \mathrm{mi}}$. & \multicolumn{2}{c|}{ Radius $=10 \mathrm{mi}}$. \\
\cline { 2 - 7 } & Median & $\begin{array}{c}\text { \# of } \\
\text { Sectors }\end{array}$ & Median & $\begin{array}{c}\text { \# of } \\
\text { Sectors }\end{array}$ & Median & $\begin{array}{c}\text { \# of } \\
\text { Sectors }\end{array}$ \\
\hline NOTIFY & 0.6 & 48 & 1.0 & 109 & 1.6 & 116 \\
PPRNC & 0.7 & 45 & 1.0 & 103 & 1.8 & 108 \\
PPRAC & 1.0 & 44 & 1.8 & 97 & 2.8 & 100 \\
TPRNC & 0.2 & 3 & 1.9 & 7 & 3.0 & 11 \\
TPRAC & 0.3 & 2 & 2.0 & 4 & 1.7 & 7 \\
GPTNC & 1.5 & 45 & 2.2 & 100 & 5.0 & 111 \\
GPTAC & 1.7 & 37 & 2.5 & 83 & 5.2 & 91 \\
CONFIRM & 0.8 & 37 & 1.0 & 74 & 2.0 & 75 \\
SPRNC & 1.5 & 9 & 2.5 & 24 & 2.7 & 44 \\
SPRAC & 2.0 & 8 & 2.3 & 18 & 3.2 & 28 \\
\hline
\end{tabular}

${ }^{\star N O T I F Y}=$ NOTIFICATION TIME

PPRNC = PERMANENT POPULATION RESPONSE TIME NORMAL CONDITIONS

PPRAC = PERMANENT POPULATION RESPONSE TIME ADVERSE CONDITIONS

TPRNC = TRANSIENT POPULATION RESPONSE TIME NORMAL CONDITIONS

TPRAC = TRANSIENT POPULATION RESPONSE TIME ADVERSE CONDITIONS

GPTNC = GENERAL POPULATION EVACUATION TIME NORMAL CONDITIONS

GPTAC = GENERAL POPULATION EVACUATION TIME ADVERSE CONDITIONS

CONFIRM = CONFIRMATION TIME

SPRNC = SPECIAL POPULATION RESPONSE TIME NORMAL CONDITIONS

SPRAC = SPECIAL POPULATION RESPONSE TIME ADVERSE CONDITIONS

SEE APPENDIX C FOR DEFINITIONS. 

FEMA ASSESSMENTS

The Federal Emergency Manayement Agency (FEMA) was requested by the Nuclear Reyulatory Commission to make several independent assessments of evacuation times around reactor sites which are located in proximity to high population densities. Initially, twelve assessments have been made by three contractors. Six of the assessments correspond to plant sites evaluated in this report. The six sites are Beaver Valley, Indian Point, Maine Yankee, Millstone, Three Mile Island, and Zion.

It should be noted that the evaluation franework prescribed by FEMA was more general than that required by the NRC. For example, the FEMA contractors were not required to follow the sector configuration specified by NRC. The zone configuration used by the FEMA contractors generally considered political subdivisions, demographic considerations and roadway locations. One contractor also used a radius of exactly 10 miles in developing time estimates. This was done to allow comparison of similar evacuation areas among the sites evaluated.

The principal difference between licensee submittals and the FEMA assessments is that the licensee submittals represented a riuch wider range of techniques. Nevertheless, some licensee submittals were in fact done by two of the FEMA contractors. The third FEMA contractor also used a subcontractor that had prepared a licensee estimate.

The methodologies used by the FEMA contractors follows closely the transportation planning techniques recommended in Reference 4. The primary difference in the FEMA estimates and the techniques used by many of the better licensee responses concerns the interaction between the various evacuation time components. 
All the FEMA contractors elected to use distributions for the various time components and combine them statistically. Two things, however, are not completely clear-cut. First, little empirical data exists for developing the distributions and that data which does exist is very limited. Second, it is not completely clear whether using distributions increases or decreases the evacuation tirie estinate. The fact that distributions allow some evacuees to begin moving should reduce total evacution time. Furthemore, one FEMA contractor concluded that gradual loading of the street system reduced total evacuation time at one site studied due to the roadway configuration. However, in some cases gradual loading could increase total evacuation times due to assumed longer times to prepare and depart, thus underutilizing roadway capacity. 


\section{CONCLUSIONS}

The results of the analysis of the evacuation time estimates provided by the various licensees defines the range of times that can reasonably be expected to occur during evacuations of the emergency planning zones. Given the variety of methodologies and assumptions used, it is ill-advised to draw any strong conclusions concerning the factors affecting evacuation time. For example, the variation in analytical methods may confound the trends observed for the effect of sector population and evacuation distance on evacuation times. However, with further refinement and consistent application of analysis techniques, it will be possible to ascertain the limiting factors in evacuation times. 

REFERENCES

1. NUREG 75/111, Guide and Checklist for Development and Evaluation of State and Local Government Radiological Emergency Response Plans in Support of Fixed Nuclear Facilities, U.S. Nuclear Regulatory Commission, Revision 1, December 1974. *

2. NUREG-0396, Planning Basis for the Development of State and Local Government Radiological Emergency Response Plans in Support of Light Water Nuclear Power Plants, U.S. Nuclear Regulatory Commission, December 1978. *

3. NUREG-0654, Criteria for Preparation and Evaluation of Radiological Emergency Response Plans and Preparedness in Support of Nuclear Power Plants (for Interim Use and Comment), U.S. Nuclear Regulatory

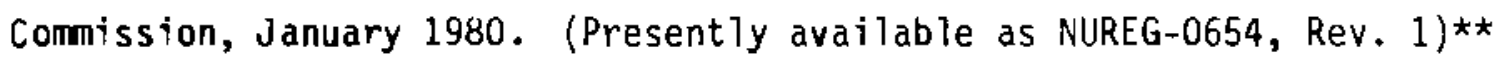

4. Thomas Urbanik, Arthur Desrosiers, Michael K. Lindell, and C. Richard Schuller, Analysis of Techniques for Estimating Evacuation Times for Emergency Planning Zones, Battelle Human Affairs Research Centers, June 1980, BHARC-401/80-017.

5. NUREG-0348, Demographic Statistics Pertaining to Nuclear Power Reactor Sites, U.S. Nuclear Regulatory Commission, October 1979. *

ॠAvailable for purchase from the National Technical Information Service, Springfield, VA 22161.

**Available free upon written request to the Division of Technical Information and Document Control, U.S. Nuclear Regulatory Commission, Washington, DC 20555. 

APPENDIX A

EVALUATION FORMS FOR INDIVIDUAL PLANTS

$A-1$ 

EVALUATION OF EVACUATION TIME ESTIMATE

Arkansas

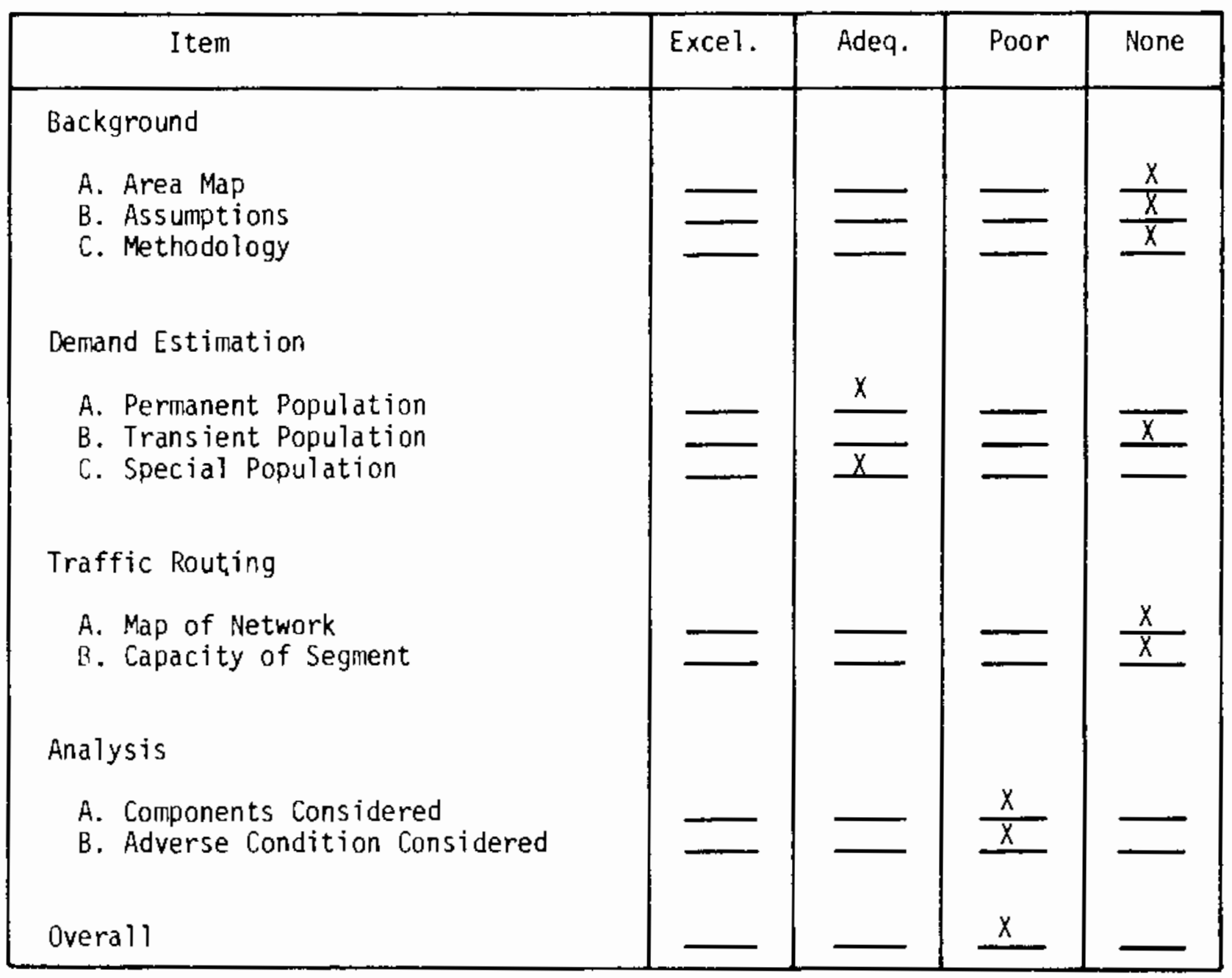




\begin{tabular}{|c|c|c|c|c|}
\hline I tem & Excel. & Adeq. & Poor & None \\
\hline Background & & & & \\
\hline $\begin{array}{l}\text { A. Area Map } \\
\text { B. Assumptions } \\
\text { C. Methodology }\end{array}$ & $\frac{X}{X}$ & $\underline{x}$ & $=$ & $\overline{-}$ \\
\hline Demand Estimation & & & & \\
\hline $\begin{array}{l}\text { A. Permanent Population } \\
\text { B. Transient Population } \\
\text { C. Special Population }\end{array}$ & - & $x$ & {[} & $\frac{x}{x}$ \\
\hline Traffic Routing & & & & \\
\hline $\begin{array}{l}\text { A. Map of Network } \\
\text { B. Capacity of Segment }\end{array}$ & - & Z & - & $\frac{x}{x}$ \\
\hline Analys is & & & & \\
\hline $\begin{array}{l}\text { A. Components Considered } \\
\text { B. Adverse Condition Considered }\end{array}$ & $\frac{x}{x}$ & & - & $=$ \\
\hline Overall & 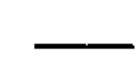 & 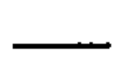 & $x$ & - \\
\hline
\end{tabular}

*Lack of complete documentation makes it difficult to assess the accuracy of the plan. The overall methodology is excellent, there is no evaluation for $90^{\circ}$ sectors. 
EVALUATION OF EVACUATION TIME ESTIMATE

Big Rock Point

\begin{tabular}{|c|c|c|c|c|}
\hline Item & Excel. & Adeq. & Poor & None \\
\hline $\begin{array}{l}\text { Background } \\
\text { A. Area Map } \\
\text { B. Assumptions } \\
\text { C. Methodology }\end{array}$ & - & $\frac{x}{x}$ & $\square$ & $\square$ \\
\hline $\begin{array}{l}\text { Demand Estimation } \\
\text { A. Permanent Population } \\
\text { B. Transient Population } \\
\text { C. Special Population }\end{array}$ & & $\begin{array}{l}X \\
X \\
X \\
\end{array}$ & & 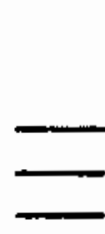 \\
\hline $\begin{array}{l}\text { Traffic Routing } \\
\text { A. Map of Network } \\
\text { B. Capacity of Segment }\end{array}$ & $x$ & $x$ & & \\
\hline $\begin{array}{l}\text { Analysis } \\
\text { A. Components Considered } \\
\text { B. Adverse Condition Considered }\end{array}$ & & $\frac{x}{x}$ & & \\
\hline Overall & 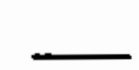 & $x$ & & \\
\hline
\end{tabular}




\begin{tabular}{|c|c|c|c|c|}
\hline Item & Exce1. & Adeq. & Poor & None \\
\hline Background & & & & \\
\hline $\begin{array}{l}\text { A. Area Map } \\
\text { B. Assumptions } \\
\text { C. Methodology }\end{array}$ & ב & 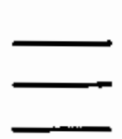 & $\bar{\square}$ & $\begin{array}{l}\frac{x}{x} \\
x \\
x\end{array}$ \\
\hline Demand Estimation & & & & \\
\hline $\begin{array}{l}\text { A. Permanent Population } \\
\text { B. Transient Population } \\
\text { C. Special Population }\end{array}$ & & X & سمس & $\frac{x}{x}$ \\
\hline Traffic Routing & & & & \\
\hline $\begin{array}{l}\text { A. Map of Network } \\
\text { B. Capacity of Segment }\end{array}$ & & - & & $\frac{x}{x}$ \\
\hline Analysis & & & & \\
\hline $\begin{array}{l}\text { A. Components Considered } \\
\text { B. Adverse Condition Considered }\end{array}$ & & - & - & $\frac{x}{x}$ \\
\hline Overall & - & & $x$ & 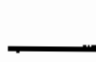 \\
\hline
\end{tabular}




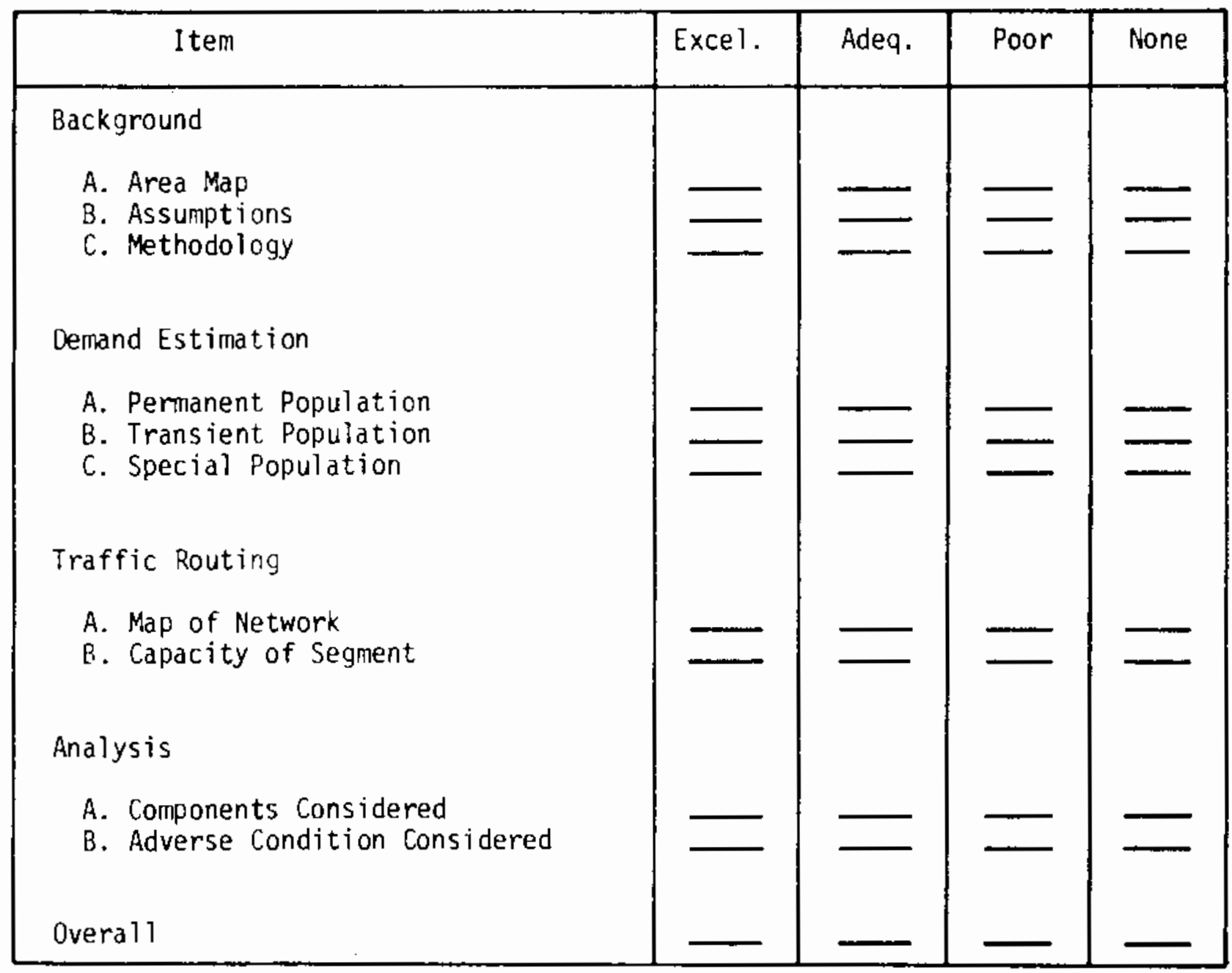

*No Response 


\section{EVALUATION OF EVACUATION TIME ESTIMATE}

Calvert Cliffs

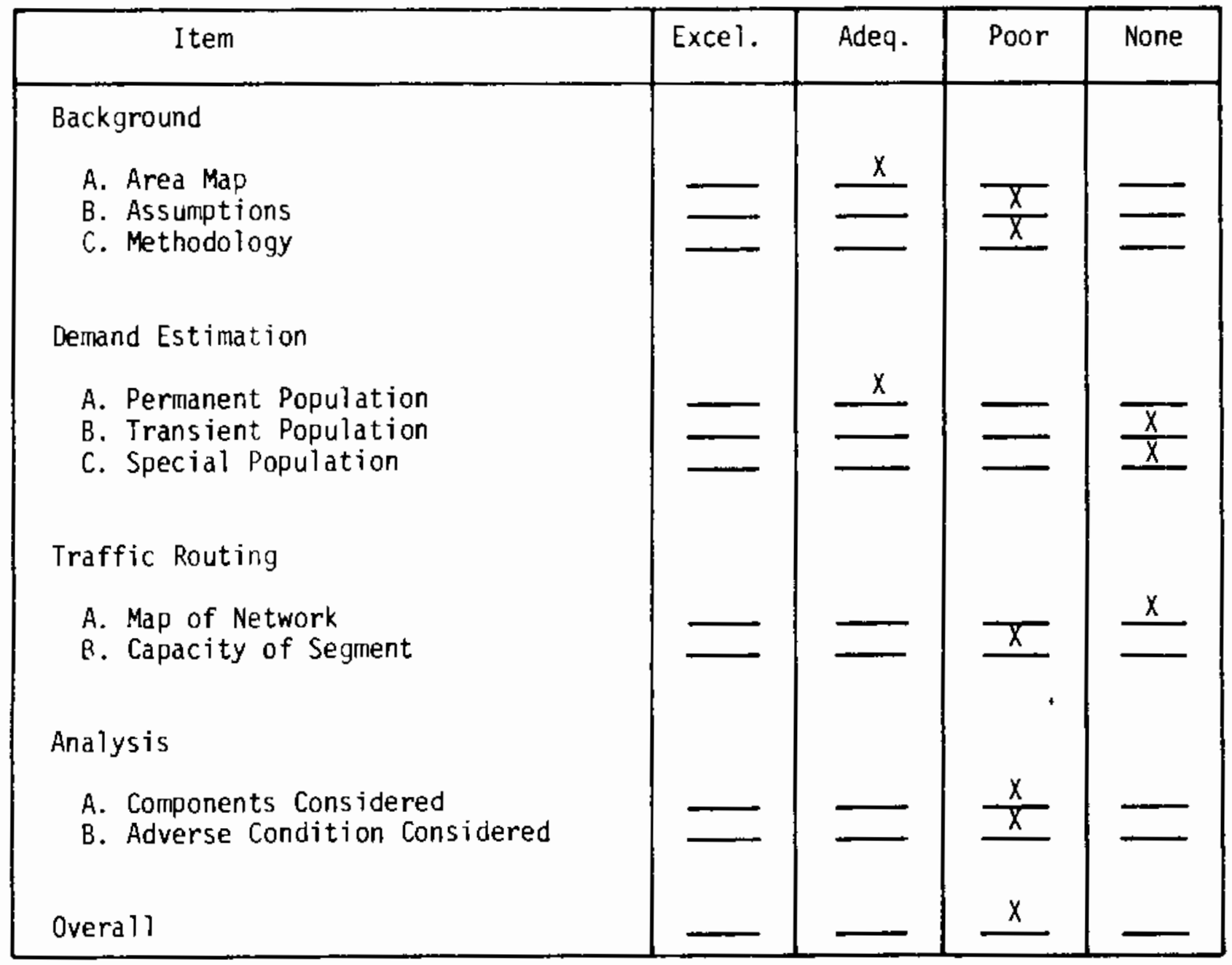




\begin{tabular}{|c|c|c|c|c|}
\hline Item & Excel. & Adeq. & Poor & None \\
\hline \multicolumn{5}{|l|}{ Background } \\
\hline A. Area Map & - & $\frac{X}{Y}$ & $\longrightarrow$ & - \\
\hline $\begin{array}{l}\text { B. Assumptions } \\
\text { C. Methodology }\end{array}$ & & $\frac{x}{x}$ & - & 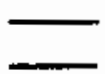 \\
\hline \multicolumn{5}{|l|}{ Demand Estimation } \\
\hline A. Permanent Population & $\underline{x}$ & $\bar{y}$ & - & - \\
\hline $\begin{array}{l}\text { B. Transient Population } \\
\text { C. Special Population }\end{array}$ & - & $\hat{n}$ & $x$ & 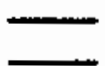 \\
\hline \multicolumn{5}{|l|}{ Traffic Routing } \\
\hline A. Map of Network & - & $\frac{x}{x}$ & - & بـــ \\
\hline \multicolumn{5}{|l|}{ Analysis } \\
\hline A. Components Considered & 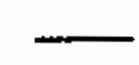 & 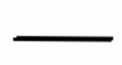 & $x$ & \\
\hline B. Adverse Condition Considered & & - & & $x$ \\
\hline Overall & & ^ & 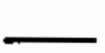 & 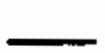 \\
\hline
\end{tabular}

time estimates provided for Nebraska side only. 


\begin{tabular}{|c|c|c|c|c|}
\hline I tem & Excel. & Adeq. & Poor & None \\
\hline Background & & & & \\
\hline $\begin{array}{l}\text { A. Area Map } \\
\text { B. Assumptions } \\
\text { C. Methodology }\end{array}$ & & $\frac{x}{x}$ & ב & $\underline{x}$ \\
\hline Demand Estimation & & & & \\
\hline $\begin{array}{l}\text { A. Permanent Population } \\
\text { B. Transient Population } \\
\text { C. Special Population }\end{array}$ & & $\frac{x}{\frac{x}{x}}$ & 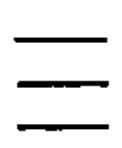 & $=$ \\
\hline Traffic Routing & & & & \\
\hline $\begin{array}{l}\text { A. Map of Network } \\
\text { B. Capacity of Segment }\end{array}$ & & $\bar{\square}$ & $\bar{Z}$ & $\begin{array}{l}x \\
x \\
\end{array}$ \\
\hline Anaiys is & & & & \\
\hline $\begin{array}{l}\text { A. Components Considered } \\
\text { B. Adverse Condition Considered }\end{array}$ & & $\frac{x}{x}$ & 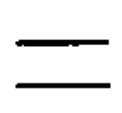 & 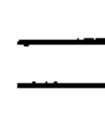 \\
\hline Overall & 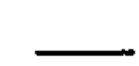 & $x$ & 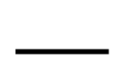 & 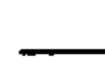 \\
\hline
\end{tabular}




\begin{tabular}{|c|c|c|c|c|}
\hline I tem & Excel. & Adeq. & Poor & None \\
\hline Background & & & & \\
\hline $\begin{array}{l}\text { A. Area Map } \\
\text { B. Assumptions } \\
\text { C. Methodology }\end{array}$ & 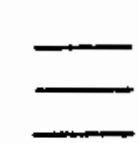 & $\underline{x}$ & $\frac{x}{x}$ & $\bar{z}$ \\
\hline Demand Estimation & & & & \\
\hline $\begin{array}{l}\text { A. Permanent Population } \\
\text { B. Transient Population } \\
\text { C. Special Population }\end{array}$ & & $\frac{x}{x}$ & $\bar{x}$ & 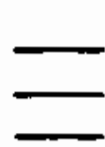 \\
\hline Traffic Routing & & & & \\
\hline $\begin{array}{l}\text { A. Map of Network } \\
\text { B. Capacity of Segment }\end{array}$ & & $\underline{x}$ & — & $x$ \\
\hline Analysis & & & & \\
\hline $\begin{array}{l}\text { A. Components Considered } \\
\text { B. Adverse Condition Considered }\end{array}$ & & $x$ & $x$ & - \\
\hline Overall & & 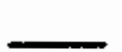 & $x$ & \\
\hline
\end{tabular}




\section{EVALUATION OF EVACUATION TIME ESTIMATE}

Donald C. Cook

\begin{tabular}{|l|l|l|l|l|}
\hline \multicolumn{1}{|c|}{ Item } & Excel. & Adeq. & Poor & None \\
\hline Background & & & & \\
A. Area Map \\
B. Assumptions \\
C. Methodology \\
Demand Estimation
\end{tabular}




\begin{tabular}{|c|c|c|c|c|}
\hline Item & Excel. & Adeq. & Poor & None \\
\hline \multicolumn{5}{|l|}{ 8ackground } \\
\hline $\begin{array}{l}\text { A. Area Map } \\
\text { B. Assumptions } \\
\text { C. Methodology }\end{array}$ & $\begin{array}{l}\frac{x}{x} \\
x \\
\end{array}$ & & ש & ב \\
\hline \multicolumn{5}{|l|}{ Demand Estimation } \\
\hline $\begin{array}{l}\text { A. Permanent Population } \\
\text { B. Transient Population } \\
\text { C. Special Population }\end{array}$ & $\begin{array}{l}\frac{x}{x} \\
x \\
\end{array}$ & & 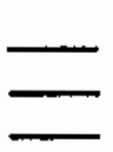 & 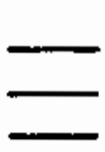 \\
\hline \multicolumn{5}{|l|}{ Traffic Routing } \\
\hline $\begin{array}{l}\text { A. Map of Network } \\
\text { B. Capacity of Segment }\end{array}$ & $\begin{array}{l}x \\
x\end{array}$ & & - & 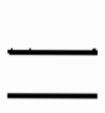 \\
\hline \multicolumn{5}{|l|}{ Analysis } \\
\hline $\begin{array}{l}\text { A. Components Considered } \\
\text { 8. Adverse Condition Considered }\end{array}$ & $\begin{array}{l}x \\
x \\
\end{array}$ & & - & 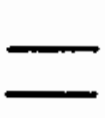 \\
\hline Overall & $x$ & & & \\
\hline
\end{tabular}




\begin{tabular}{|c|c|c|c|c|}
\hline I tem & Excel. & Adeq. & Poor & None \\
\hline \multicolumn{5}{|l|}{ Background } \\
\hline $\begin{array}{l}\text { A. Area Map } \\
\text { B. Assumptions } \\
\text { C. Methodology }\end{array}$ & 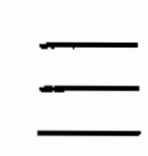 & $\frac{\frac{x}{x}}{x}$ & $\bar{Z}$ & 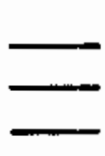 \\
\hline \multicolumn{5}{|l|}{ Demand Estimation } \\
\hline $\begin{array}{l}\text { A. Permanent Population } \\
\text { B. Transient Population } \\
\text { C. Special Population }\end{array}$ & - & $\begin{array}{l}\frac{x}{x} \\
x \\
\end{array}$ & & 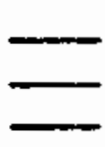 \\
\hline \multicolumn{5}{|l|}{ Traffic Routing } \\
\hline $\begin{array}{l}\text { A. Map of Network } \\
\text { B. Capacity of Segment }\end{array}$ & & $\frac{x}{x}$ & & 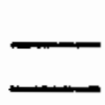 \\
\hline \multicolumn{5}{|l|}{ Analysis } \\
\hline $\begin{array}{l}\text { A. Components Considered } \\
\text { B. Adverse Condition Considered }\end{array}$ & & $x$ & $x$ & - \\
\hline Overall & & $x$ & - & \\
\hline
\end{tabular}




\begin{tabular}{|c|c|c|c|c|}
\hline Item & Excel. & Adeq. & Poor & None \\
\hline Background & & & & \\
\hline $\begin{array}{l}\text { A. Area Map } \\
\text { B. Assumptions } \\
\text { C. Methodology }\end{array}$ & 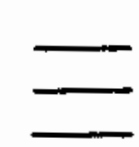 & $\begin{array}{l}\frac{x}{x} \\
x \\
\end{array}$ & ב & $=$ \\
\hline Denand Estimation & & & & \\
\hline $\begin{array}{l}\text { A. Permanent Population } \\
\text { B. Transient Population } \\
\text { C. Special Population }\end{array}$ & $x$ & $x$ & & $\bar{x}$ \\
\hline Traffic Routing & & & & \\
\hline $\begin{array}{l}\text { A. Map of Network } \\
\text { B. Capacity of Segment }\end{array}$ & - & $\frac{x}{x}$ & & _ \\
\hline Analysis & & & & \\
\hline $\begin{array}{l}\text { A. Components Considered } \\
\text { B. Adverse Condition Considered }\end{array}$ & - & $\frac{x}{x}$ & 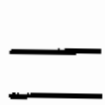 & - \\
\hline Overall & 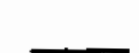 & $x$ & & \\
\hline
\end{tabular}




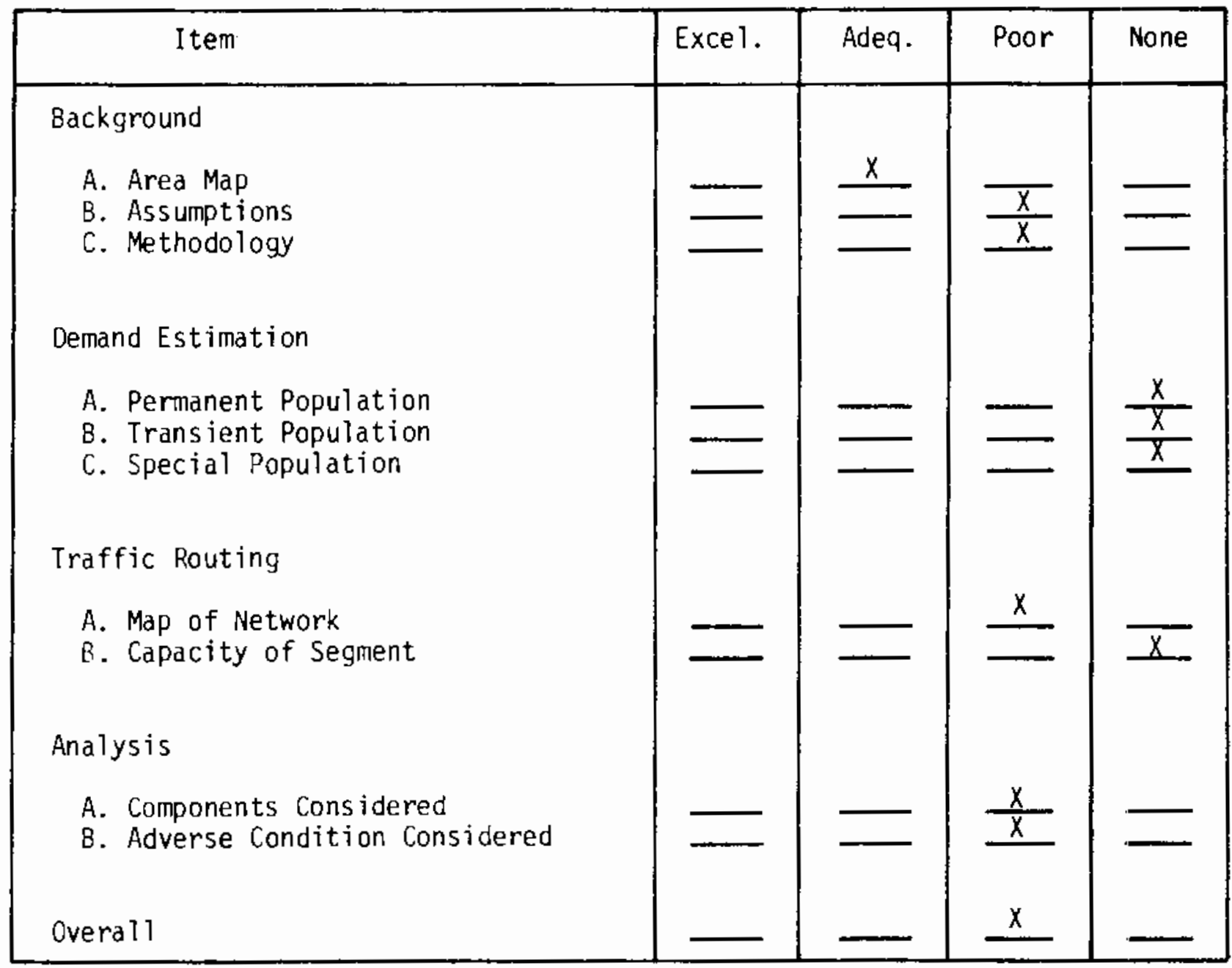




\section{EVALUATION OF EVACUATION TIME ESTIMATE}

Fitzpatrick ${ }^{l}$

\begin{tabular}{|c|c|c|c|c|}
\hline I tem & Excel. & Adeq. & Poor & None \\
\hline \multicolumn{5}{|l|}{ Background } \\
\hline A. Area Map & 7 & $\lambda$ & - & - \\
\hline C. Methodology & $x$ & - & & {[} \\
\hline \multicolumn{5}{|l|}{ Demand Estimation } \\
\hline A. Permanent Population & _- & $\frac{x}{x}$ & - & $\overline{-}$ \\
\hline C. Special Population & - & $X$ & & \\
\hline \multicolumn{5}{|l|}{ Traffic Routing } \\
\hline A. Map of Network & & $\underline{X}$ & & 2 \\
\hline \multicolumn{5}{|l|}{ Analysis } \\
\hline A. Components Considered & - & $\frac{x}{x}$ & $\ldots$ & 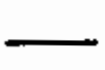 \\
\hline Overall & 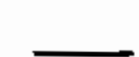 & $\mathrm{x}$ & 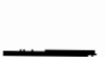 & 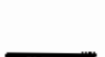 \\
\hline
\end{tabular}

ISame location as Nine Mile Point.

${ }^{2}$ Adequate methodology, not actually reported. 


\begin{tabular}{|c|c|c|c|c|}
\hline I tem & Exce 1. & Adeq. & Poor & None \\
\hline $\begin{array}{l}\text { Background } \\
\text { A. Area Map } \\
\text { B. Assumptions } \\
\text { C. Methodology }\end{array}$ & 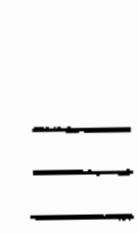 & 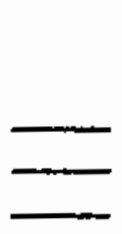 & $\frac{\frac{x}{x}}{\frac{x}{x}}$ & - \\
\hline $\begin{array}{l}\text { Demand Estimation } \\
\text { A. Permanent Population } \\
\text { B. Transient Population } \\
\text { C. Special Population }\end{array}$ & 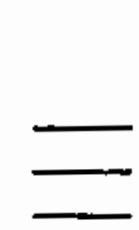 & $\frac{x}{x}$ & $=$ & $\underline{x}$ \\
\hline $\begin{array}{l}\text { Traffic Routing } \\
\text { A. Map of Network } \\
\text { B. Capacity of Segment }\end{array}$ & & $x$ & & . \\
\hline $\begin{array}{l}\text { Analysis } \\
\text { A. Components Considered } \\
\text { B. Adverse Condition Considered }\end{array}$ & & & $\begin{array}{l}\frac{x}{x} \\
\end{array}$ & \\
\hline Overall & - & & $x^{*}$ & \\
\hline
\end{tabular}

* Note: The rating is based on data submitted for Nebraska side. An estimate was provided for the Iowa side with little documentation. The Iowa rating would be poor for all items. 
Ft. St, Vrain

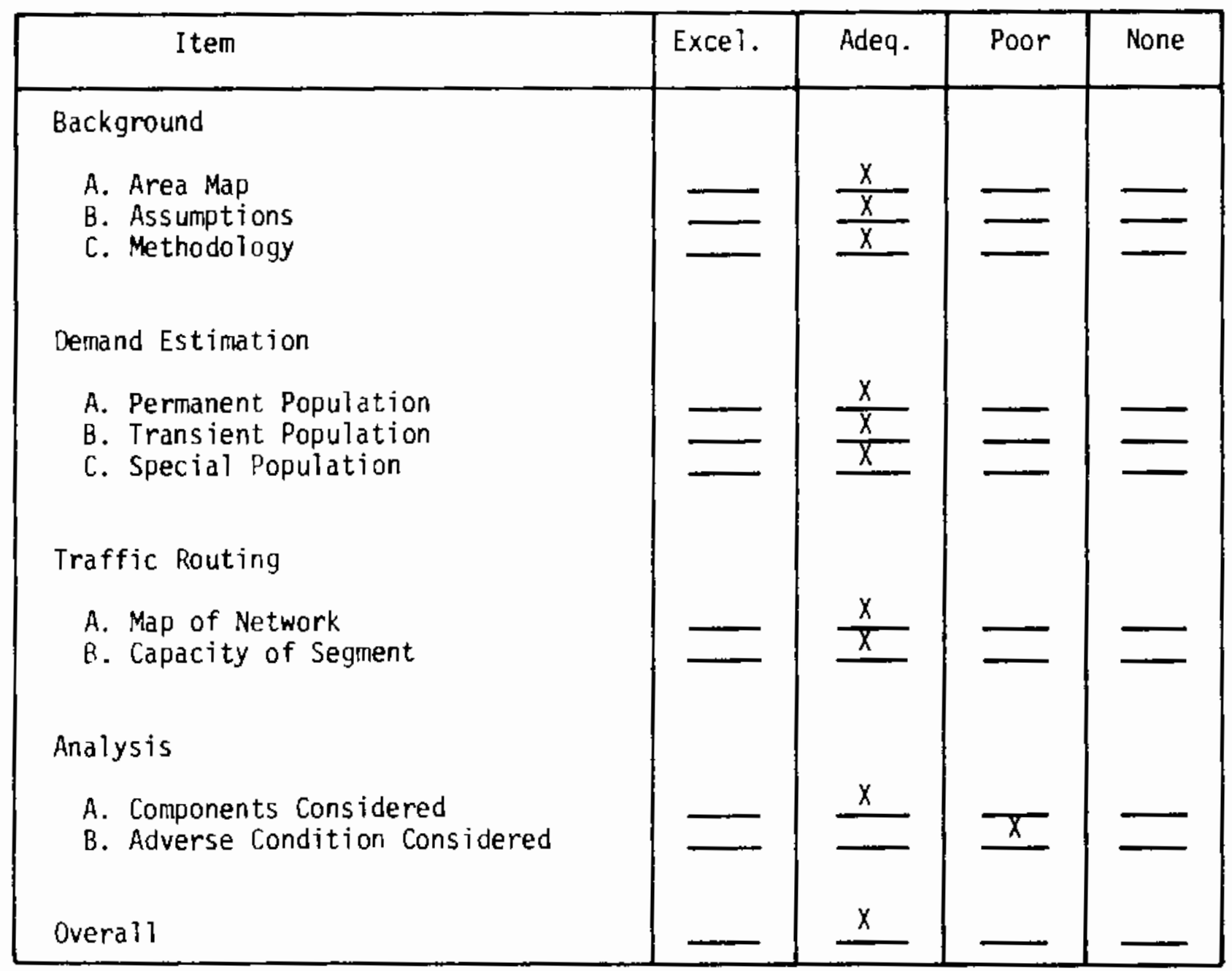




\begin{tabular}{|c|c|c|c|c|}
\hline Item & Excel. & Adeq. & Poor & None \\
\hline \multicolumn{5}{|l|}{ Background } \\
\hline A. Area Map & $\frac{x}{x}$ & - & $\longrightarrow$ & - \\
\hline B. Assumptions & $\frac{x}{x}$ & - & - & - \\
\hline \multicolumn{5}{|l|}{ Demand Estimation } \\
\hline A. Permanent Population & - & $\frac{x}{x}$ & & $\longrightarrow$ \\
\hline c. Special Population & . & $x$ & & - \\
\hline \multicolumn{5}{|l|}{ Traffic Routing } \\
\hline A. Map of Network & & $x$ & $x$ & \\
\hline \multicolumn{5}{|l|}{ Analysis } \\
\hline $\begin{array}{l}\text { A. Components Considered } \\
\text { B. Adverse Condition Considered }\end{array}$ & - & $\frac{x}{x}$ & - & - \\
\hline Overall & & $x$ & & \\
\hline
\end{tabular}




\begin{tabular}{|c|c|c|c|c|}
\hline Item & Excel. & Adeq. & Poor & None \\
\hline \multicolumn{5}{|l|}{ 8ackground } \\
\hline A. Area Map & & $\underline{x}$ & $\ldots$ & - \\
\hline c. Methodology & 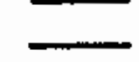 & $x$ & - & _ \\
\hline \multicolumn{5}{|l|}{ Demand Estimation } \\
\hline A. Permanent Population & $\frac{x}{y}$ & - & & \\
\hline C. Special Population & $\underline{X}$ & & & \\
\hline \multicolumn{5}{|l|}{ Traffic Routing } \\
\hline A. Map of Network & $\frac{x}{x}$ & 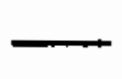 & & \\
\hline \multicolumn{5}{|l|}{ Analysis } \\
\hline $\begin{array}{l}\text { A. Components Considered } \\
\text { B. Adverse Condition Considered }\end{array}$ & $\bar{X}$ & $X$ & & \\
\hline Overall & 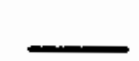 & $x$ & & \\
\hline
\end{tabular}


Hatch

\begin{tabular}{|c|c|c|c|c|}
\hline I tem & Excel. & Adeq. & Poor & None \\
\hline Background & & & & \\
\hline $\begin{array}{l}\text { A. Area Map } \\
\text { B. Assumptions } \\
\text { C. Methodology }\end{array}$ & & ב & $x$ & $\bar{x}$ \\
\hline Demand Estimation & & & & \\
\hline $\begin{array}{l}\text { A. Permanent Population } \\
\text { B. Transient Population } \\
\text { C. Special Population }\end{array}$ & & $\frac{x}{x}$ & 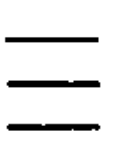 & $\bar{x}$ \\
\hline Traffic Routing & & & & \\
\hline $\begin{array}{l}\text { A. Map of Network } \\
\text { B. Capacity of Segment }\end{array}$ & & $\underline{x}$ & & $x$ \\
\hline Analys is & & & & \\
\hline $\begin{array}{l}\text { A. Components Considered } \\
\text { B. Adverse Condition Considered }\end{array}$ & & $\begin{array}{l}x \\
x \\
\end{array}$ & & $\bar{Z}$ \\
\hline Overall & 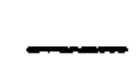 & 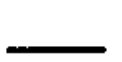 & $x$ & \\
\hline
\end{tabular}




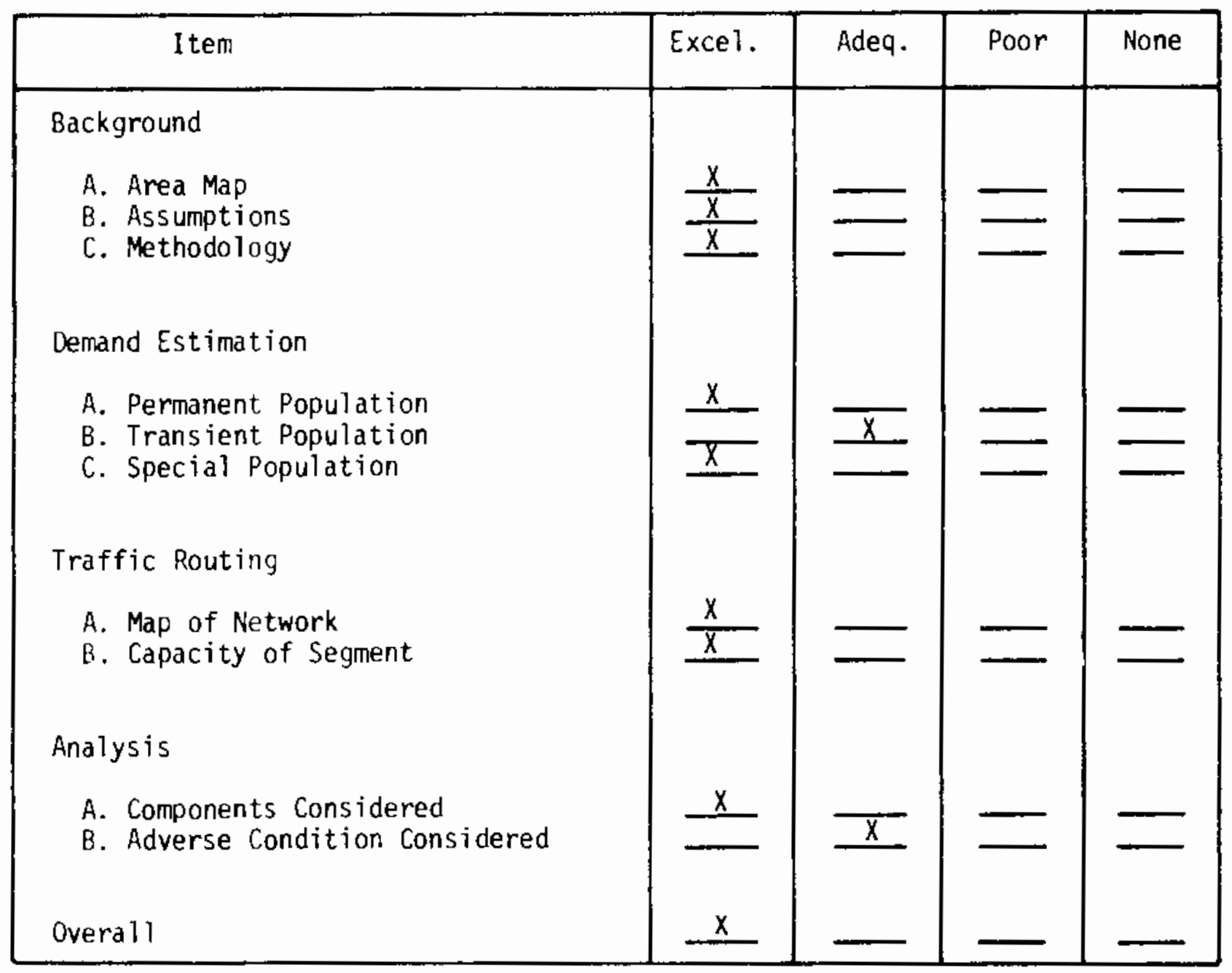




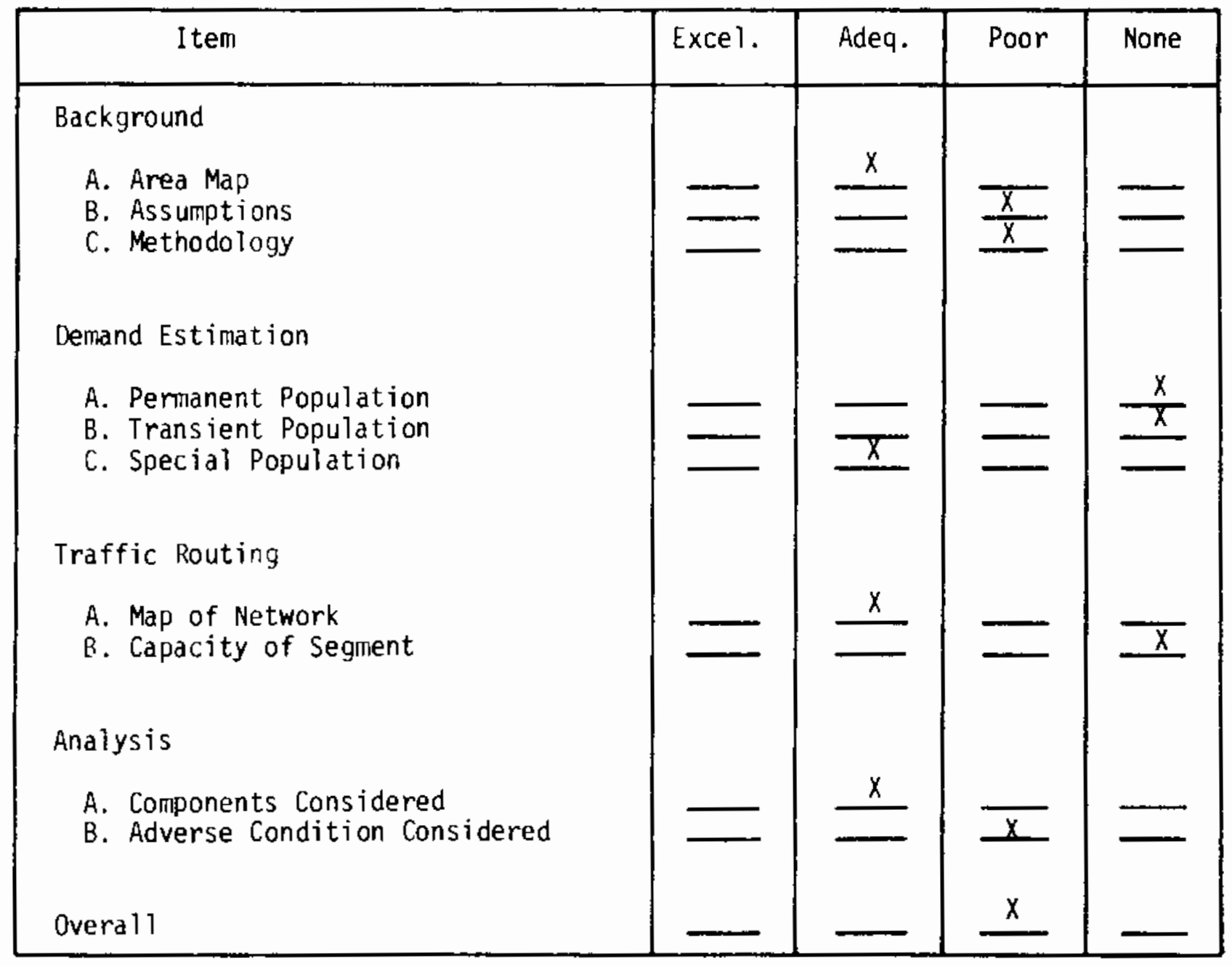




\begin{tabular}{|c|c|c|c|c|}
\hline I tem & Excel. & Adeq. & Poor & None \\
\hline Background & & & & \\
\hline $\begin{array}{l}\text { A. Area Map } \\
\text { B. Assumptions } \\
\text { C. Methodology }\end{array}$ & {[} & $=$ & $\frac{\frac{x}{x}}{\frac{x}{x}}$ & ב \\
\hline Demand Estimation & & & & \\
\hline $\begin{array}{l}\text { A. Permanent Population } \\
\text { B. Transient Population } \\
\text { C. Special Population }\end{array}$ & & $\bar{x}$ & $\frac{x}{x}$ & - \\
\hline Traffic Routing & & & & \\
\hline $\begin{array}{l}\text { A. Map of Network } \\
\text { B. Capacity of Segment }\end{array}$ & - & & $\underline{x}$ & $x$ \\
\hline Analysis & & & & \\
\hline $\begin{array}{l}\text { A. Components Considered } \\
\text { B. Adverse Condition Considered }\end{array}$ & & $x$ & $\bar{x}$ & {[} \\
\hline Overall & & . & $x$ & \\
\hline
\end{tabular}




\begin{tabular}{|c|c|c|c|c|}
\hline I tem & Excel. & Adeq. & Poor & None \\
\hline Background & & & & \\
\hline $\begin{array}{l}\text { A. Area Map } \\
\text { B. Assumptions } \\
\text { C. Methodology }\end{array}$ & & $\frac{\frac{x}{x}}{x}$ & $=$ & $\bar{Z}$ \\
\hline Demand Estimation & & & & \\
\hline $\begin{array}{l}\text { A. Permanent Population } \\
\text { B. Transient Population } \\
\text { C. Special Population }\end{array}$ & & $\frac{x}{x}$ & & 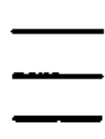 \\
\hline Traffic Routing & & & & \\
\hline $\begin{array}{l}\text { A. Map of Network } \\
\text { B. Capacity of Segment }\end{array}$ & & $\frac{x}{x}$ & & \\
\hline Analysis & & & & \\
\hline $\begin{array}{l}\text { A. Components Considered } \\
\text { B. Adverse Condition Considered }\end{array}$ & & $x$ & $X$ & \\
\hline Overall & & $x$ & - & \\
\hline
\end{tabular}




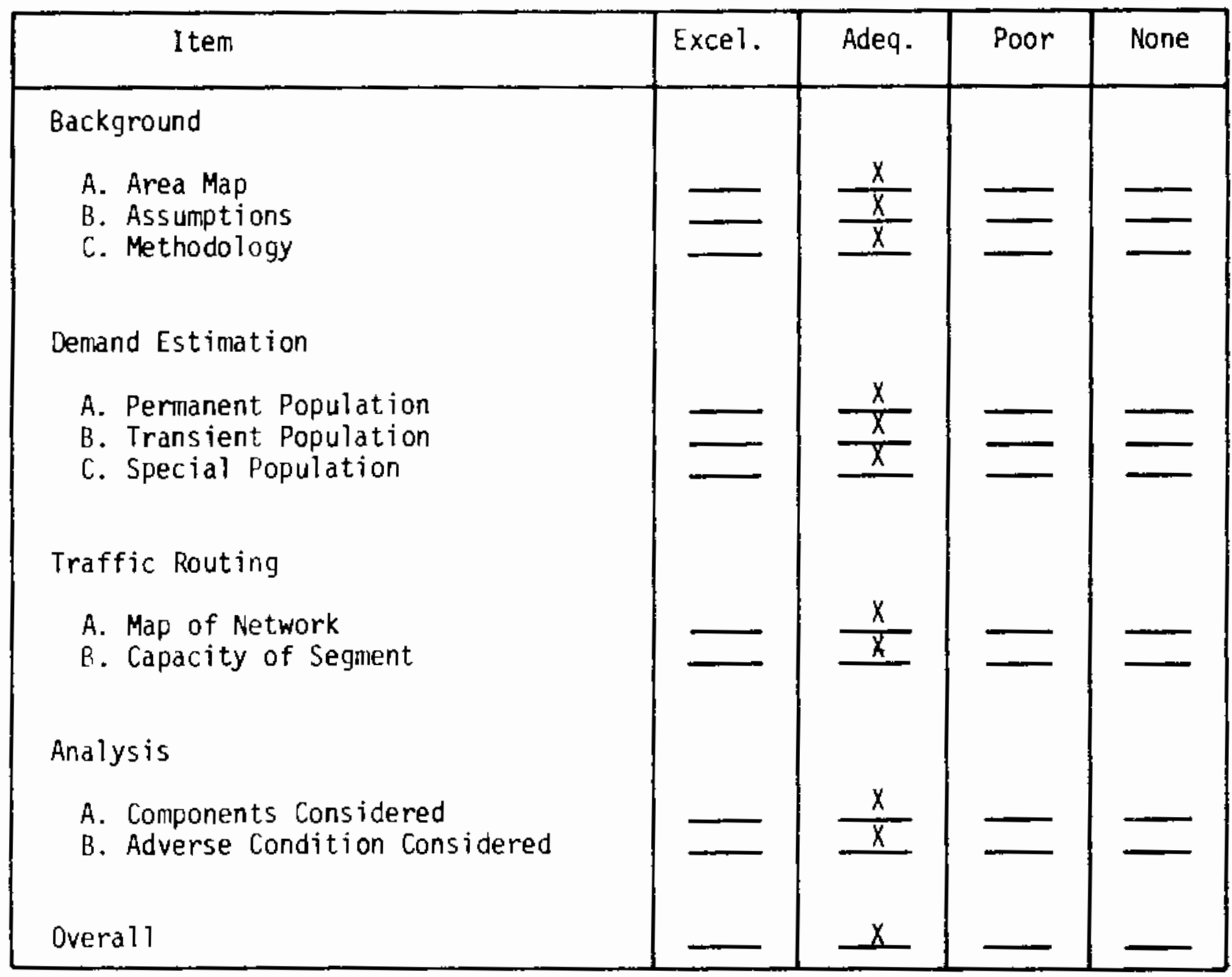

Note: Data not reported in detajl. 


\begin{tabular}{|c|c|c|c|c|}
\hline I tem & Excel. & Adeq. & Poor & None \\
\hline Background & & & & \\
\hline $\begin{array}{l}\text { A. Area Map } \\
\text { B. Assumptions } \\
\text { C. Methodology }\end{array}$ & - & $\frac{x}{x}$ & & $\begin{array}{l}x \\
\\
\end{array}$ \\
\hline Demand Estimation & & & & \\
\hline $\begin{array}{l}\text { A. Permanent Population } \\
\text { B. Transient Population } \\
\text { C. Special Population }\end{array}$ & $\begin{array}{l}\frac{x}{x} \\
x \\
\end{array}$ & - & & 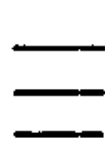 \\
\hline Traffic Routing & & & & \\
\hline $\begin{array}{l}\text { A. Map of Network } \\
\text { B. Capacity of Segment }\end{array}$ & 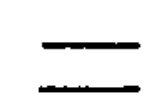 & $x$ & & $x$ \\
\hline Anaiysis & & & & \\
\hline $\begin{array}{l}\text { A. Components Considered } \\
\text { B. Adverse Condition Considered }\end{array}$ & - & $\frac{x}{x}$ & & $\ldots$ \\
\hline Overall & . & $x$ & & - \\
\hline
\end{tabular}

Note: Results not reported in cummulative format as requested. 


\begin{tabular}{|c|c|c|c|c|}
\hline Item & Excel. & Adeq. & Poor & None \\
\hline \multicolumn{5}{|l|}{ Background } \\
\hline $\begin{array}{l}\text { A. Area Map } \\
\text { B. Assumptions } \\
\text { C. Methodology }\end{array}$ & $x$ & $\frac{x}{x}$ & & - \\
\hline \multicolumn{5}{|l|}{ Demand Estimation } \\
\hline $\begin{array}{l}\text { A. Permanent Population } \\
\text { B. Transient Population } \\
\text { C. Special Population }\end{array}$ & $\frac{x}{x}$ & - & & ב \\
\hline \multicolumn{5}{|l|}{ Traffic Routing } \\
\hline $\begin{array}{l}\text { A. Map of Network } \\
\text { B. Capacity of Segment }\end{array}$ & $\frac{x}{x}$ & & & - \\
\hline \multicolumn{5}{|l|}{ Analysis } \\
\hline $\begin{array}{l}\text { A. Components Considered } \\
\text { B. Adverse Condition Considered }\end{array}$ & $\bar{X}$ & $\underline{x}$ & & \\
\hline Overall & - & $x$ & - & \\
\hline
\end{tabular}




\begin{tabular}{|c|c|c|c|c|}
\hline Item & Excel. & Adeq. & Poor & None \\
\hline \multicolumn{5}{|l|}{ Background } \\
\hline $\begin{array}{l}\text { A. Area Map } \\
\text { B. Assumptions } \\
\text { C. Methodology }\end{array}$ & & $\frac{x}{\frac{x}{x}}$ & $\bar{Z}$ & - \\
\hline \multicolumn{5}{|l|}{ Demand Estimation } \\
\hline $\begin{array}{l}\text { A. Permanent Population } \\
\text { B. Transient Population } \\
\text { C. Special Population }\end{array}$ & - & $\frac{x}{x}$ & & 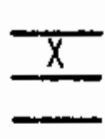 \\
\hline \multicolumn{5}{|l|}{ Traffic Routing } \\
\hline $\begin{array}{l}\text { A. Map of Network } \\
\text { 8. Capacity of Segment }\end{array}$ & & $\begin{array}{l}x \\
x \\
\end{array}$ & & - \\
\hline \multicolumn{5}{|l|}{ Analysis } \\
\hline $\begin{array}{l}\text { A. Components Considered } \\
\text { B. Adverse Condition Considered }\end{array}$ & & $\begin{array}{l}x \\
x \\
\end{array}$ & & 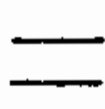 \\
\hline Overali & - & $x$ & 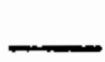 & - \\
\hline
\end{tabular}

Note: Some capacities reported appear high. 
EVALUATION OF EVACUATION TIME ESTIMATE

Nỉne Mile Point ${ }^{1}$

\begin{tabular}{|c|c|c|c|c|}
\hline I tem & Excel. & Adeq. & Poor & None \\
\hline Background & & & & \\
\hline $\begin{array}{l}\text { A. Area Map } \\
\text { B. Assumptions } \\
\text { C. Methodology }\end{array}$ & $\frac{x}{x}$ & $\underline{x}$ & $=$ & $=$ \\
\hline Dèmand Estimation & & & & \\
\hline $\begin{array}{l}\text { A. Permanent Population } \\
\text { B. Transient Population } \\
\text { C. Special Population }\end{array}$ & 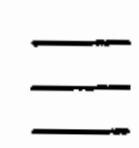 & $\frac{\frac{x}{x}}{x}$ & - & $\longrightarrow$ \\
\hline Traffic Routing & & & & \\
\hline $\begin{array}{l}\text { A. Map of Network } \\
\text { B. Capacity of Segment }\end{array}$ & - & $x$ & & 2 \\
\hline Analysis & & & & \\
\hline $\begin{array}{l}\text { A. Components Considered } \\
\text { B. Adverse Condition Considered }\end{array}$ & - & $-\frac{x}{x}$ & 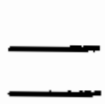 & - \\
\hline Overal & & $x$ & 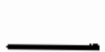 & \\
\hline
\end{tabular}

${ }^{1}$ Same location as Fitzpatrick.

${ }^{2}$ Adequate methodology, not actualiy reported. 


\begin{tabular}{|l|l|l|l|l|}
\hline \multicolumn{1}{|c|}{ Item } & Excel. & Adeq. & Poor & None \\
\hline Background & & & & \\
A. Area Map \\
B. Assumptions \\
C. Methodology \\
Demand Estimation
\end{tabular}

*It is not possible to assess the overall validity of the estimates since there is no information on assumptions or methodology. 
EVALUATION OF EVACUATION TIME ESTIMATE

Oconee

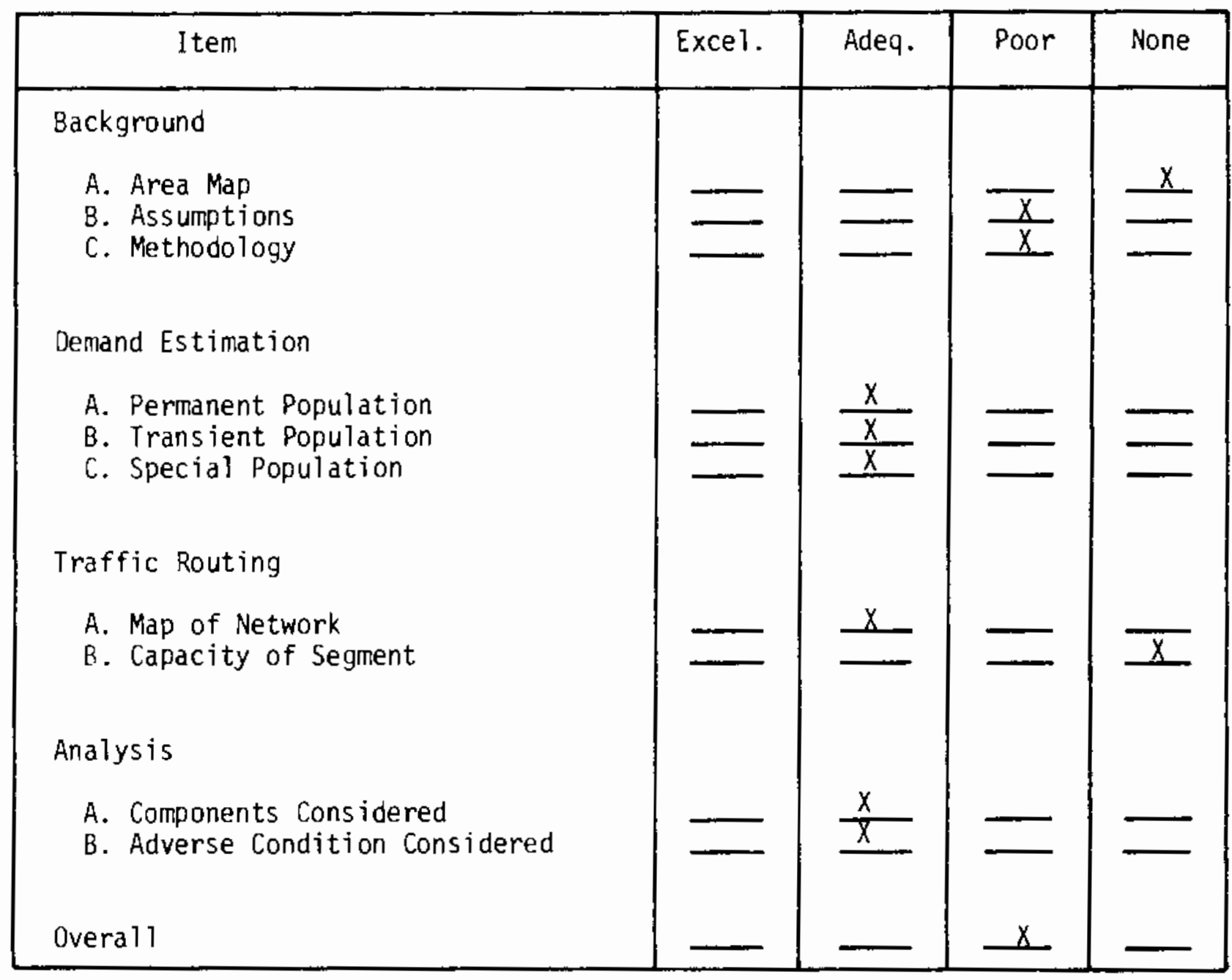




\begin{tabular}{|c|c|c|c|c|}
\hline I tem & Excel. & Adeq. & Poor & None \\
\hline $\begin{array}{l}\text { Background } \\
\text { A. Area Map } \\
\text { B. Assumptions } \\
\text { C. Methodology }\end{array}$ & $=$ & $\frac{\frac{x}{x}}{x}$ & $\bar{Z}$ & 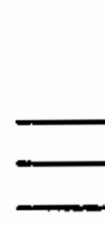 \\
\hline $\begin{array}{l}\text { Demand Estimation } \\
\text { A. Permanent Population } \\
\text { B. Transient Population } \\
\text { C. Special Population }\end{array}$ & 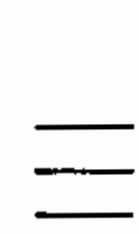 & $\bar{Z}$ & - & $\frac{\star}{X}$ \\
\hline $\begin{array}{l}\text { Traffic Routing } \\
\text { A. Map of Network } \\
\text { B. Capacity of Segment }\end{array}$ & & $\frac{x}{x}$ & & - \\
\hline $\begin{array}{l}\text { Analysis } \\
\text { A. Components Considered } \\
\text { B. Adverse Condition Considered }\end{array}$ & & & & \\
\hline Overall & & $\underline{x}$ & - & יבוב \\
\hline
\end{tabular}

* Population data considered, but not reported. 


\begin{tabular}{|c|c|c|c|c|}
\hline I tem & Excer. & Adeq. & Poor & None \\
\hline $\begin{array}{l}\text { Background } \\
\text { A. Area Map } \\
\text { B. Assumptions } \\
\text { C. Methodology }\end{array}$ & $=$ & $\frac{X}{X}$ & 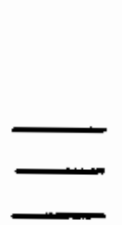 & 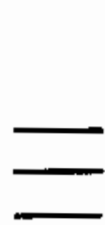 \\
\hline $\begin{array}{l}\text { Demand Estimation } \\
\text { A. Permanent Population } \\
\text { B. Transient Population } \\
\text { C. Special Population }\end{array}$ & & $\frac{x}{x}$ & $\bar{x}$ & 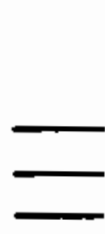 \\
\hline $\begin{array}{l}\text { Traffic Routing } \\
\text { A. Map of Network } \\
\text { B. Capacity of Segment }\end{array}$ & $\frac{x}{x}$ & 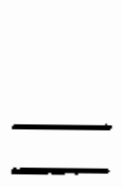 & 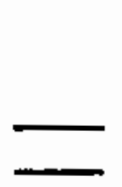 & \\
\hline $\begin{array}{l}\text { Analysis } \\
\text { A. Components Considered } \\
\text { B. Adverse Condition Considered }\end{array}$ & - & $x$ & $\bar{x}$ & \\
\hline Overail & - & $\underline{x}$ & - & \\
\hline
\end{tabular}




\begin{tabular}{|c|c|c|c|c|}
\hline I tem & Exce 1. & Adeq. & Poor & None \\
\hline \multicolumn{5}{|l|}{ Background } \\
\hline A. Area Map & - & & - & $x$ \\
\hline B. Assumptions & & $\frac{x}{x}$ & $\longrightarrow$ & $\longrightarrow$ \\
\hline \multicolumn{5}{|l|}{ Demand Estimation } \\
\hline $\begin{array}{l}\text { A. Permanent Population } \\
\text { B. Transient Population }\end{array}$ & & _ & & $-\frac{\hbar}{x}$ \\
\hline C. Special Population & & $x$ & & - \\
\hline \multicolumn{5}{|l|}{ Traffic Routing } \\
\hline A. Map of Network & & & & $x$ \\
\hline B. Capacity of Segment & & $x$ & & 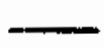 \\
\hline \multicolumn{5}{|l|}{ Analysis } \\
\hline $\begin{array}{l}\text { A. Components Considered } \\
\text { B. Adverse Condition Considered }\end{array}$ & & $\frac{x}{x}$ & & - \\
\hline Dverall & & $x$ & 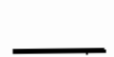 & 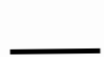 \\
\hline
\end{tabular}

*Not reported, although considered in analysis. 


\begin{tabular}{|c|c|c|c|c|}
\hline item & Excel. & Adeq. & Poor & None \\
\hline \multicolumn{5}{|l|}{ Background } \\
\hline A. Area Map & $x$ & & $\underline{x}$ & - \\
\hline $\begin{array}{l}\text { B. Assumptions } \\
\text { c. Methodology }\end{array}$ & $\frac{n}{x}$ & 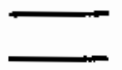 & - & $\bar{Z}$ \\
\hline \multicolumn{5}{|l|}{ Demand Estimation } \\
\hline A. Permanent Population & $\frac{x}{y}$ & & - & $\longrightarrow$ \\
\hline $\begin{array}{l}\text { B. Transient Population } \\
\text { C. Special Population }\end{array}$ & $-\frac{\lambda}{x}$ & & - & $\overline{-}$ \\
\hline \multicolumn{5}{|l|}{ Traffic Routing } \\
\hline $\begin{array}{l}\text { A. Map of Network } \\
\text { B. Capacity of Segment }\end{array}$ & $\frac{x}{x}$ & & - & בـ \\
\hline \multicolumn{5}{|l|}{ Analysis } \\
\hline A. Components Considered & $\bar{x}$ & $x$ & 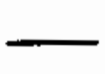 & $\ldots$ \\
\hline Overa\}l & $x$ & 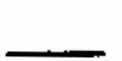 & 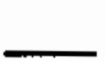 & - \\
\hline
\end{tabular}




\begin{tabular}{|c|c|c|c|c|}
\hline I tem & Excel. & Adeq. & Poor & None \\
\hline Background & & & & \\
\hline $\begin{array}{l}\text { A. Area Map } \\
\text { B. Assumptions } \\
\text { C. Methodology }\end{array}$ & {[} & $\frac{x}{\frac{x}{x}}$ & ב & $\bar{W}$ \\
\hline Demand Estimation & & & & \\
\hline $\begin{array}{l}\text { A. Permanent Population } \\
\text { B. Transient Population } \\
\text { C. Special Population }\end{array}$ & & $\begin{array}{l}\frac{X}{X} \\
X \\
\end{array}$ & & \\
\hline Traffic Routing & & & & \\
\hline $\begin{array}{l}\text { A. Map of Network } \\
\text { B. Capacity of Segment }\end{array}$ & & $x$ & & $\bar{x}$ \\
\hline Anailys is & & & & \\
\hline $\begin{array}{l}\text { A. Components Considered } \\
\text { B. Adverse Condition Considered }\end{array}$ & & $\frac{x}{x}$ & & \\
\hline Overa 11 & & $x$ & {[} & - \\
\hline
\end{tabular}




\section{EVALUATION OF EVACUATION TIME ESTIMATE}

Prajrie Island

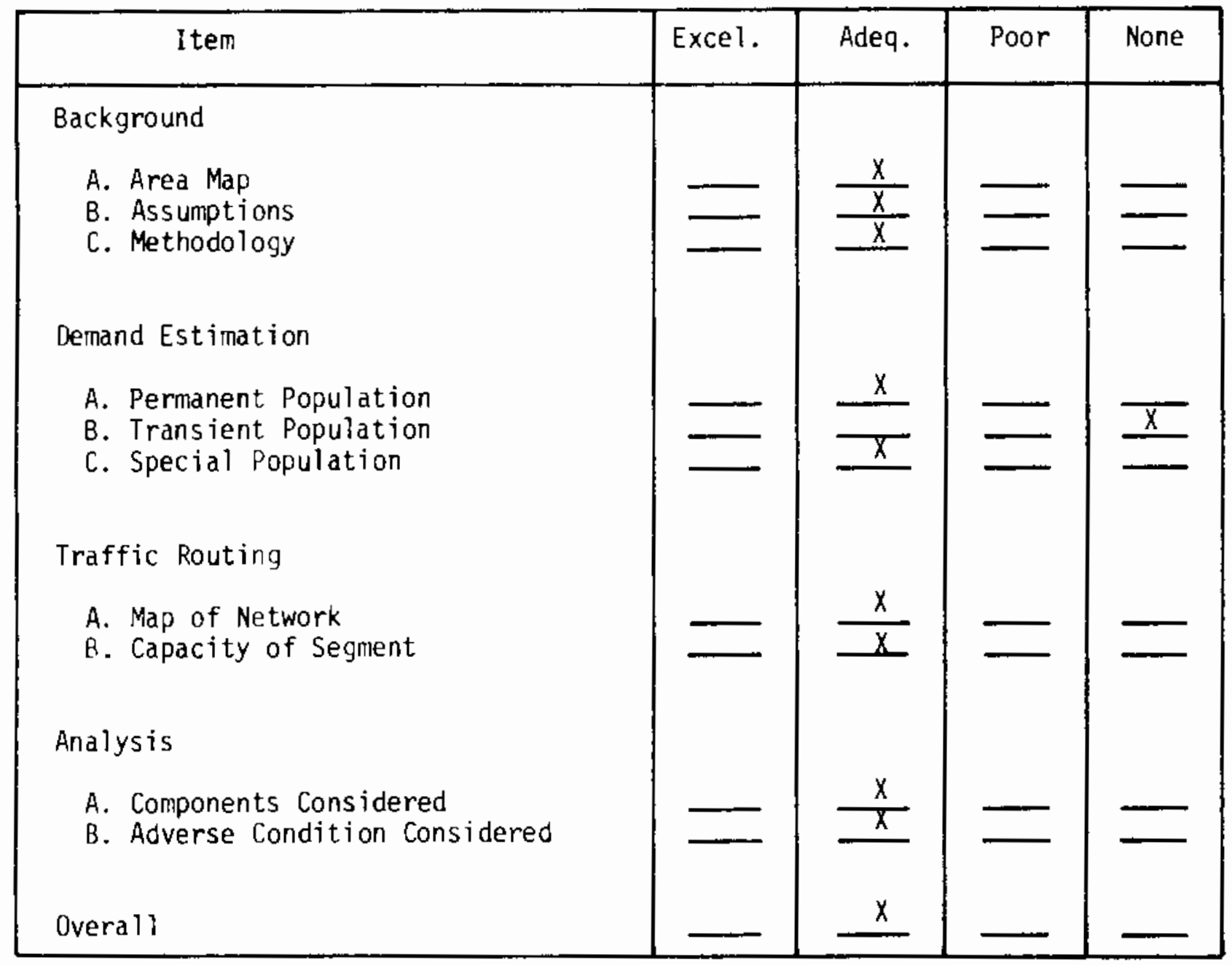




\begin{tabular}{|l|l|l|l|l|}
\hline \multicolumn{1}{|c|}{ Item } & Excel. & Adeq. & Poor & None \\
\hline Background & & & & \\
A. Area Map \\
B. Assumptions \\
C. Methodology \\
Dernand Estimation
\end{tabular}




\begin{tabular}{|c|c|c|c|c|}
\hline Item & Excel. & Adeq. & Poor & None \\
\hline \multicolumn{5}{|l|}{ Background } \\
\hline A. Area Map & $\frac{x}{y}$ & & - & $\Longrightarrow$ \\
\hline B. Assumptions & $\frac{x}{x}$ & & - & - \\
\hline \multicolumn{5}{|l|}{ Demand Estimation } \\
\hline A. Permanent Population & $\frac{x}{y}$ & - & — & 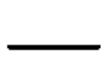 \\
\hline $\begin{array}{l}\text { B. Transient Population } \\
\text { C. Special Population }\end{array}$ & $\hat{x}$ & & - & $=$ \\
\hline \multicolumn{5}{|l|}{ Traffic Routing } \\
\hline $\begin{array}{l}\text { A. Map of Network } \\
\text { B. Capacity of Segment }\end{array}$ & - & $\frac{x}{x}$ & & - \\
\hline \multicolumn{5}{|l|}{ Analysis } \\
\hline $\begin{array}{l}\text { A. Components Considered } \\
\text { B. Adverse Condition Considered }\end{array}$ & $\begin{array}{l}x \\
x \\
\end{array}$ & 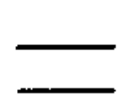 & _ & 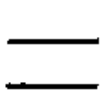 \\
\hline Overall & $x$ & 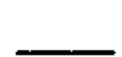 & 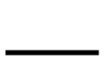 & 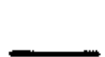 \\
\hline
\end{tabular}




\begin{tabular}{|l|l|l|l|l|}
\hline \multicolumn{1}{|c|}{ Item } & Excel. & Adeq. & Poor & None \\
\hline Background & & & & \\
A. Area Map \\
B. Assumptions \\
C. Methodology \\
Demand Estimation \\
A. Permanent Population \\
B. Transient Population \\
C. Special Population \\
Traffic Routing \\
A. Map of Network \\
B. Capacity of Segmient \\
Analysis
\end{tabular}

*No Response 
EVALUATION OF EVACUATION TIME ESTIMATE

Saint Lucie

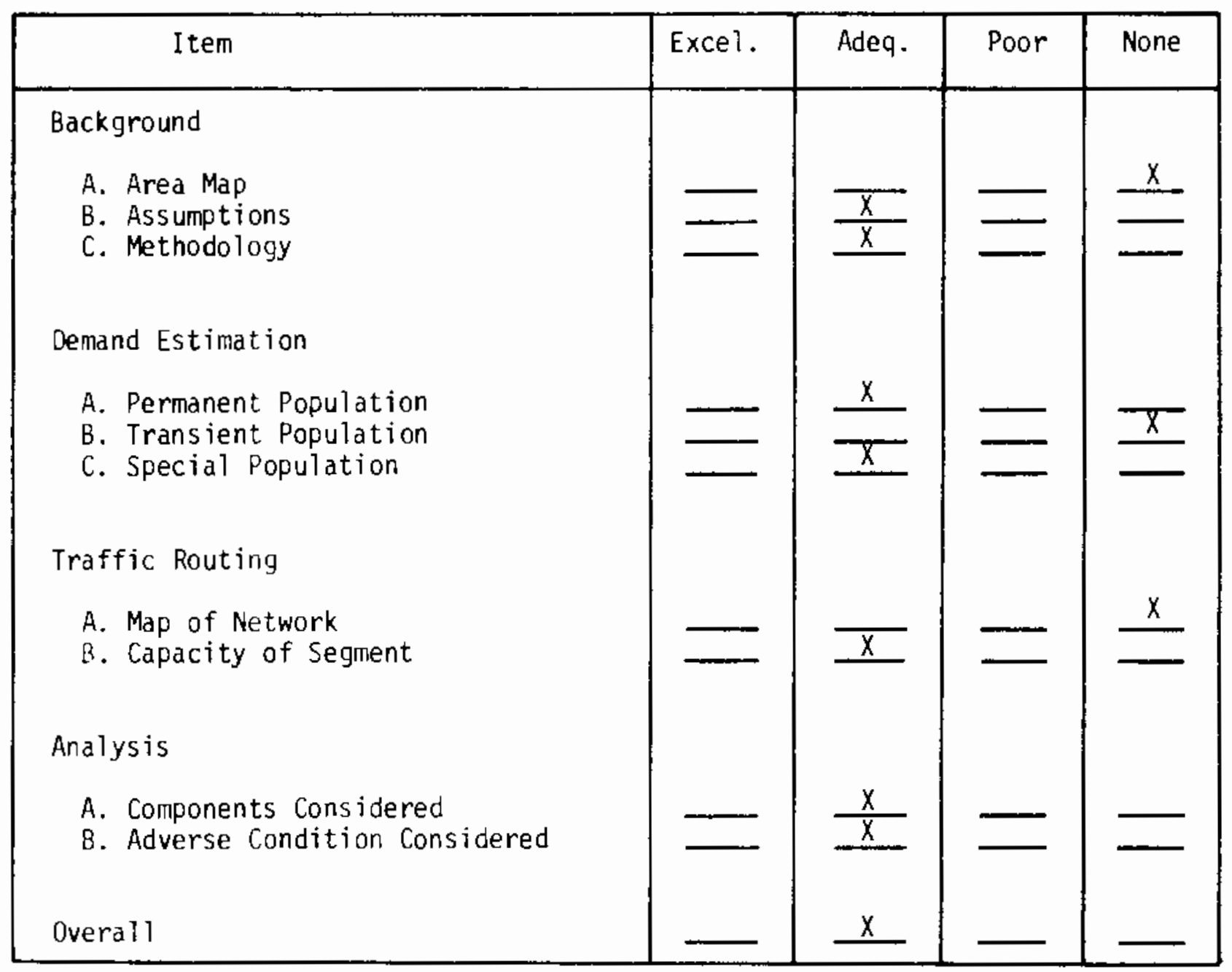




\begin{tabular}{|c|c|c|c|c|}
\hline Item & Excel. & Adeq. & Poor & None \\
\hline \multicolumn{5}{|l|}{ Background } \\
\hline $\begin{array}{l}\text { A. Area Map } \\
\text { B. Assumptions } \\
\text { C. Methodology }\end{array}$ & W & $\underline{x}$ & $\frac{x}{x}$ & ב. \\
\hline \multicolumn{5}{|l|}{ Demand Estimation } \\
\hline $\begin{array}{l}\text { A. Permanent Population } \\
\text { B. Transient Population } \\
\text { C. Specia? Population }\end{array}$ & $x$ & $=$ & ב & $\frac{x}{x}$ \\
\hline \multicolumn{5}{|l|}{ Traffic Routing } \\
\hline $\begin{array}{l}\text { A. Map of Network } \\
\text { B. Capacity of Segment }\end{array}$ & & & $\underline{x}$ & $x$ \\
\hline \multicolumn{5}{|l|}{ Analysis } \\
\hline $\begin{array}{l}\text { A. Components Considered } \\
\text { 8. Adverse Condition Considered }\end{array}$ & & & $\frac{x}{x}$ & - \\
\hline Overa11 & 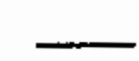 & $\underline{-}$ & $x$ & $\ldots$ \\
\hline
\end{tabular}

* A revised evacuation time estimate, which was received after the deadline for inclusion in this analysis, has been evaluated and rated excellent. 


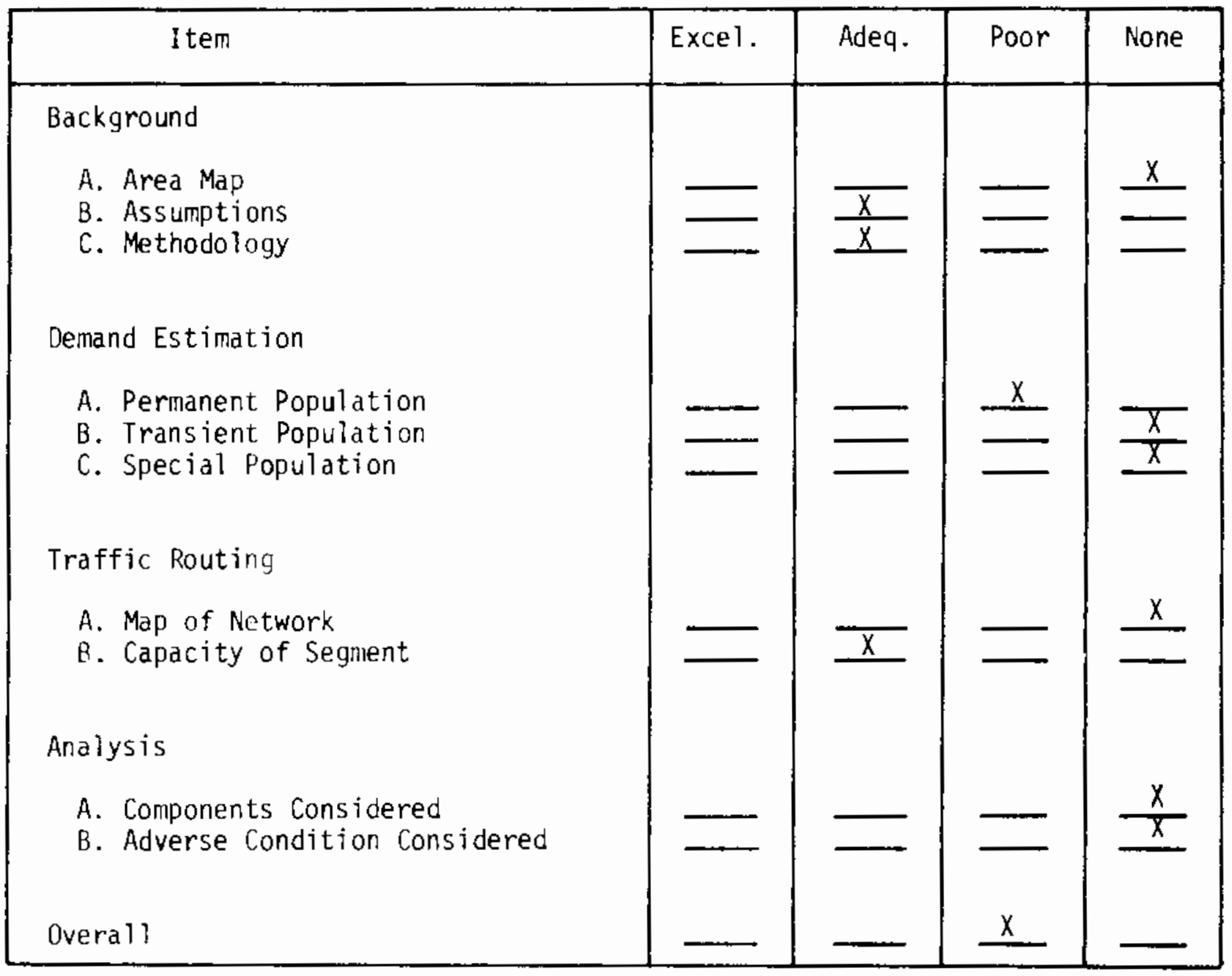




\begin{tabular}{|c|c|c|c|c|}
\hline Item & Exce?. & Adeq. & Poor & None \\
\hline Background & & & & \\
\hline $\begin{array}{l}\text { A. Area Map } \\
\text { B. Assumptions } \\
\text { C. Methodology }\end{array}$ & $\longrightarrow$ & $\bar{x}$ & $\bar{x}$ & $-\frac{x}{-2}$ \\
\hline Demand Estimation & & & & \\
\hline $\begin{array}{l}\text { A. Permanent Population } \\
\text { B. Transient Population } \\
\text { C. Special Population }\end{array}$ & & 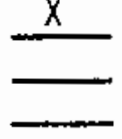 & ב & $\frac{x}{x}$ \\
\hline Traffic Routing & & & & \\
\hline $\begin{array}{l}\text { A. Map of Network } \\
\text { B. Capacity of Segment }\end{array}$ & & $=$ & $\bar{x}$ & $x$ \\
\hline Analysis & & & & \\
\hline $\begin{array}{l}\text { A. Components Considered } \\
\text { B. Adverse Condition Considered }\end{array}$ & & $\underline{x}$ & $\bar{x}$ & - \\
\hline Overa!l & . & 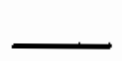 & $x$ & 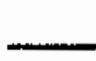 \\
\hline
\end{tabular}




\begin{tabular}{|c|c|c|c|c|}
\hline Item & Excel. & Adeq. & Poor & None \\
\hline Background & & & & \\
\hline $\begin{array}{l}\text { A. Area Map } \\
\text { B. Assumptions } \\
\text { C. Methodology }\end{array}$ & {[} & $\stackrel{x}{\square}$ & $=$ & $\frac{\bar{x}}{x}$ \\
\hline Demand Estimation & & & & \\
\hline $\begin{array}{l}\text { A. Permanent Population } \\
\text { B. Transient Population } \\
\text { C. Special Population }\end{array}$ & & $\begin{array}{l}x \\
X \\
X \\
\end{array}$ & & ב \\
\hline Traffic Routing & & & & \\
\hline $\begin{array}{l}\text { A. Map of Network } \\
\text { B. Capacity of Segment }\end{array}$ & & $x$ & & $x$ \\
\hline Analys is & & & & \\
\hline $\begin{array}{l}\text { A. Components Considered } \\
\text { B. Adverse Condition Considered }\end{array}$ & & $\frac{X}{X}$ & & 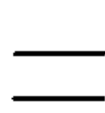 \\
\hline Overall & & 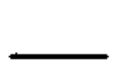 & $x$ & \\
\hline
\end{tabular}

*It is not possible to assess the overall validity of the estimates since there is no information on assumptions or methodology. 
EVALUATION OF EVACUATION TIME ESTIMATE

Three Mile Island

\begin{tabular}{|c|c|c|c|c|}
\hline Item & Excel. & Adeq. & Poor & None \\
\hline \multicolumn{5}{|l|}{ Background } \\
\hline A. Area Map & - & & - & $x$ \\
\hline $\begin{array}{l}\text { B. Assumptions } \\
\text { C. Methodology }\end{array}$ & $\longrightarrow$ & $x$ & $x$ & 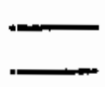 \\
\hline \multicolumn{5}{|l|}{ Demand Estimation } \\
\hline A. Permanent Population & - & - & — & $\frac{x}{x}$ \\
\hline $\begin{array}{l}\text { B. Transient Population } \\
\text { c. Special Population }\end{array}$ & & $x$ & $\longrightarrow$ & $x$ \\
\hline \multicolumn{5}{|l|}{ Traffic Routing } \\
\hline $\begin{array}{l}\text { A. Map of Network } \\
\text { B. Capacity of Segment }\end{array}$ & - & $\longrightarrow$ & - & $\frac{x}{x}$ \\
\hline \multicolumn{5}{|l|}{ Analysis } \\
\hline $\begin{array}{l}\text { A. Components Considered } \\
\text { B. Adverse Condition Considered }\end{array}$ & - & - & $\frac{x}{x}$ & - \\
\hline Overall & & & $x$ & \\
\hline
\end{tabular}


EVALUATION OF EVACUATION TIME ESTIMATE

Trojan

\begin{tabular}{|c|c|c|c|c|}
\hline Item & Excel. & Adeq. & Poor & None \\
\hline \multicolumn{5}{|l|}{ Background } \\
\hline A. Area Map & $\ldots$ & $\frac{x}{x}$ & & \\
\hline C. Methodology & $X$ & & 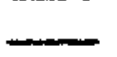 & - \\
\hline \multicolumn{5}{|l|}{ Demand Estimation } \\
\hline A. Permanent Population & $X$ & - & - & \\
\hline C. Special Population & $X$ & & & - \\
\hline \multicolumn{5}{|l|}{ Traffic Routing } \\
\hline A. Map of Network & $x$ & $\bar{x}$ & & \\
\hline \multicolumn{5}{|l|}{ Analysis } \\
\hline A. Components Considered & $\frac{x}{x}$ & 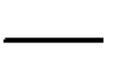 & & \\
\hline B. Adverse Conaition Considered & A- & & & \\
\hline Overali & $\underline{x}$ & & & \\
\hline
\end{tabular}




\section{EVALUATION OF EVACUATION TIME ESTIMATE}

Turkey Point

\begin{tabular}{|l|l|l|l|l|}
\hline \multicolumn{1}{|c|}{ Item } & Excel. & Adeq. & Poor & None \\
\hline Background & & & & \\
A. Area Map \\
B. Assumptions \\
C. Methodology \\
Demand Estimation \\
$\begin{array}{l}\text { A. Permanent Population } \\
\text { B. Transient Population } \\
\text { C. Special Population } \\
\text { Traffic Routing }\end{array}$ \\
$\begin{array}{l}\text { A. Map of Network } \\
\text { B. Capacity of Segment } \\
\text { Analysis }\end{array}$ \\
$\begin{array}{l}\text { A. Components Considered } \\
\text { B. Adverse Condition Considered }\end{array}$ \\
Overall
\end{tabular}




\begin{tabular}{|c|c|c|c|c|}
\hline Item & Excel. & Adeq. & Poor & None \\
\hline \multicolumn{5}{|l|}{ Background } \\
\hline A. Area Map & $X$ & & & \\
\hline $\begin{array}{l}\text { B. Assumptions } \\
\text { C. Methodology }\end{array}$ & $\bar{x}$ & $x$ & - & 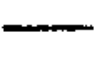 \\
\hline \multicolumn{5}{|l|}{ Demand Estimation } \\
\hline A. Permanent Population & - & $\bar{y}$ & & $x$ \\
\hline C. Special Population & & $\bar{X}$ & & \\
\hline \multicolumn{5}{|l|}{ Traffic Routing } \\
\hline A. Map of Network & $\underline{x}$ & 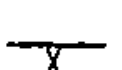 & & \\
\hline \multicolumn{5}{|l|}{ Analysis } \\
\hline A. Components Considered & . & $\frac{x}{x}$ & & \\
\hline Overall & & $x$ & & \\
\hline
\end{tabular}




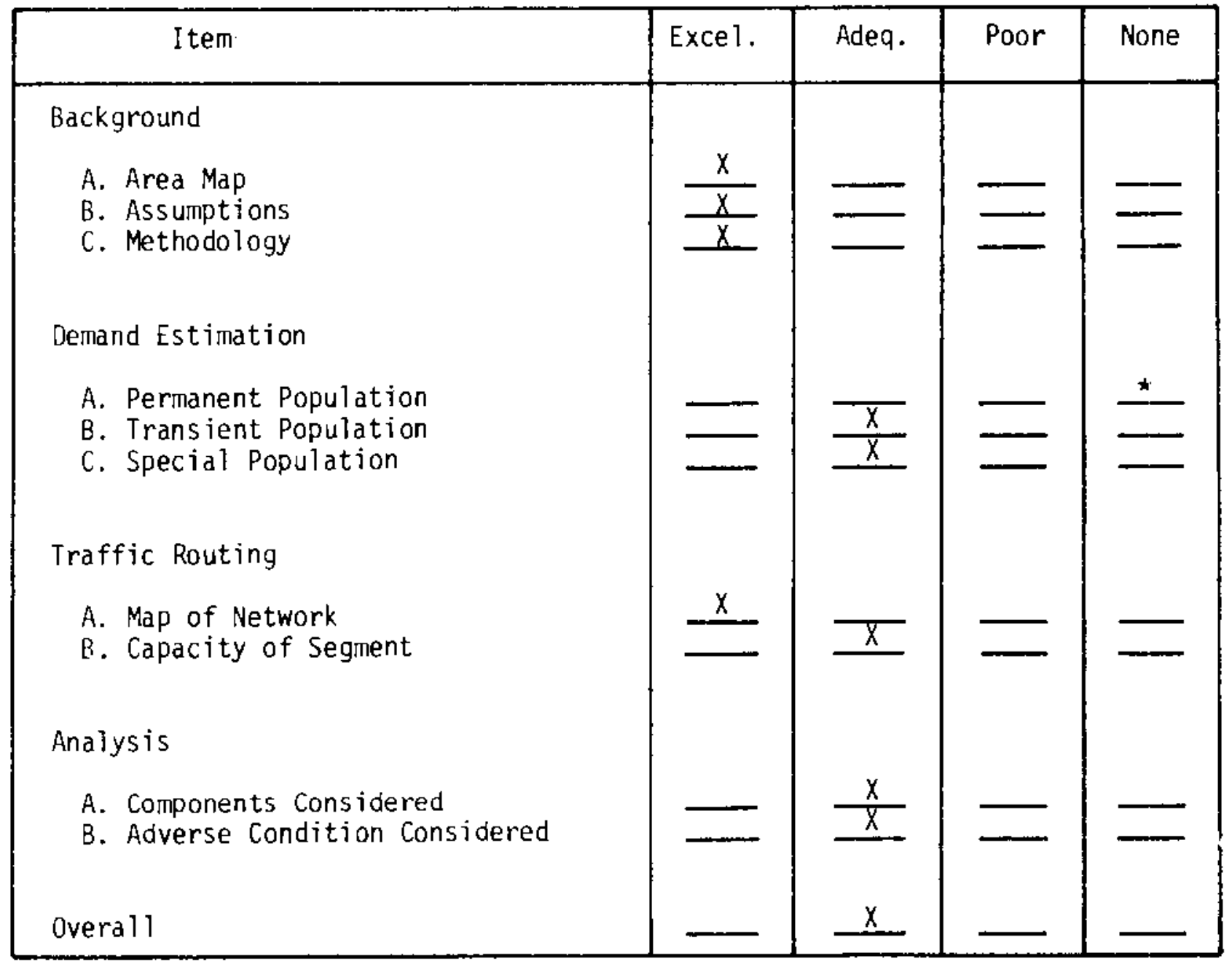

*Not reported, but obviously considered. 


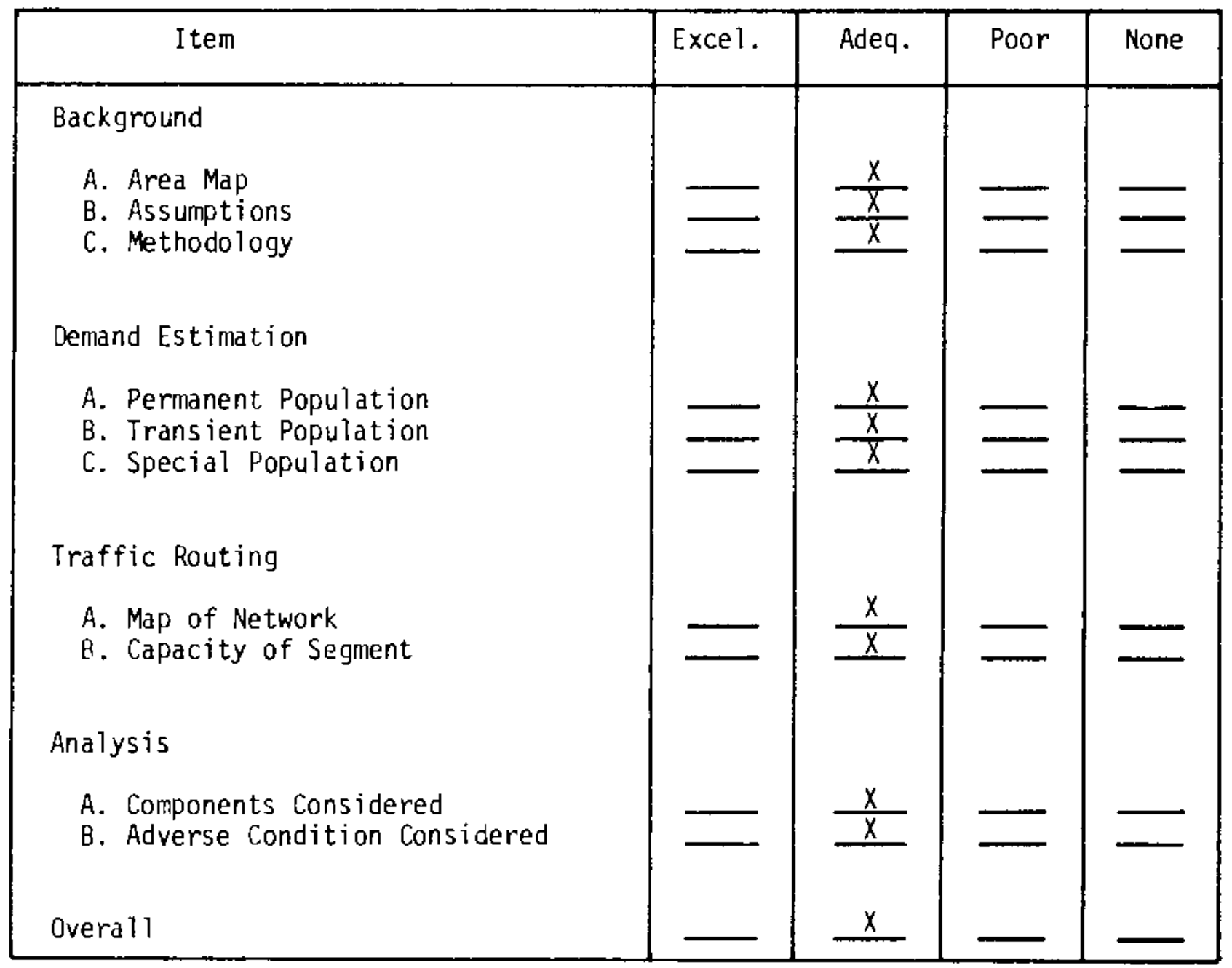




\begin{tabular}{|c|c|c|c|c|}
\hline Item & Excel. & Adeq. & Poor & None \\
\hline \multicolumn{5}{|l|}{ Background } \\
\hline A. Area Map & 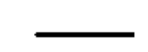 & $x$ & - & - \\
\hline $\begin{array}{l}\text { B. Assumptions } \\
\text { C. Methodology }\end{array}$ & & $\frac{x}{x}$ & 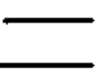 & $\overline{-}$ \\
\hline \multicolumn{5}{|l|}{ Demand Estimation } \\
\hline A. Permanent Population & - & $\frac{x}{y}$ & 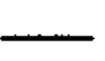 & — \\
\hline c. Special Population & & $x$ & & $=$ \\
\hline \multicolumn{5}{|l|}{ Traffic Routing } \\
\hline $\begin{array}{l}\text { A. Map of Network } \\
\text { R. Capacity of Segment }\end{array}$ & - & $\frac{x}{x}$ & $\ldots$ & - \\
\hline \multicolumn{5}{|l|}{ Analysis } \\
\hline $\begin{array}{l}\text { A. Components Considered } \\
\text { B. Adverse Condition Considered }\end{array}$ & & $x$ & $\bar{x}$ & \\
\hline Overall & & $x$ & 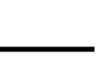 & - \\
\hline
\end{tabular}


APPENDIX B

SUMMARY OF EVACUATION TIME ESTIMATES

FOR INDIVIDUAL PLANTS

B-1 



\begin{tabular}{|c|c|c|c|c|c|c|c|c|c|c|c|c|c|c|c|c|}
\hline 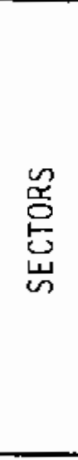 & 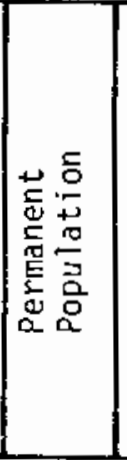 & 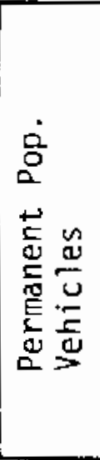 & 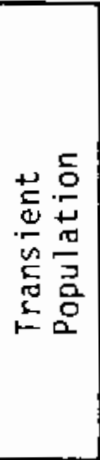 & 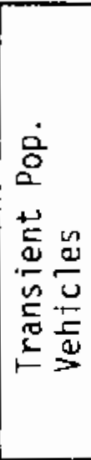 & 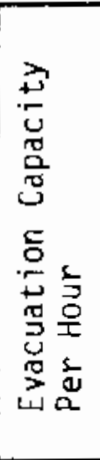 & 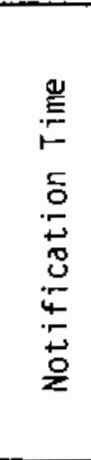 & 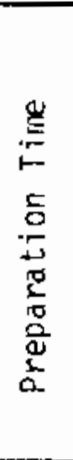 & 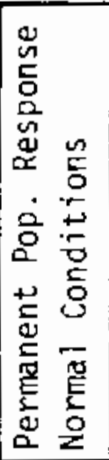 & 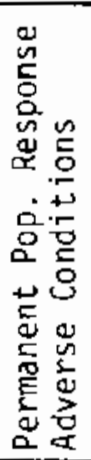 & 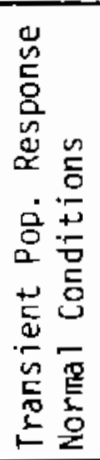 & 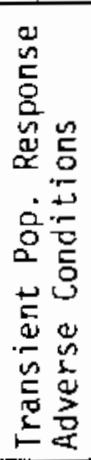 & 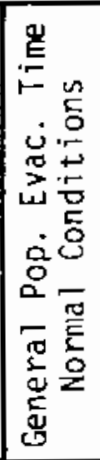 & 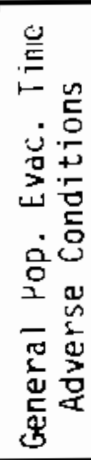 & 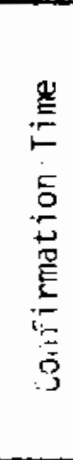 & 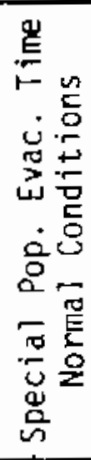 & 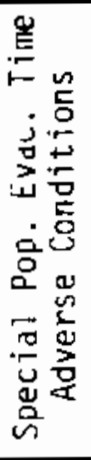 \\
\hline \multicolumn{17}{|c|}{ WITHIN TWO MILES } \\
\hline$N$ & 624 & & & & & 30 & & & 60 & & & & 90 & 60 & & \\
\hline$S$ & 31 & & & & & 30 & & & 60 & & & & 90 & 60 & & \\
\hline \multicolumn{17}{|c|}{ WITHIN FIVE MILES } \\
\hline NE & 1288 & & & & & 60 & & & 60 & & & & 120 & 90 & & \\
\hline SE & 5623 & & & & & 60 & & & 120 & & & & 180 & 90 & 360 & \\
\hline SW & 421 & & & & & 60 & & & 60 & & & & 180 & 90 & & \\
\hline $\mathrm{NW}$ & 1023 & & & & & 60 & & & 60 & & & & 120 & 90 & & \\
\hline \multicolumn{17}{|c|}{ WITHIN TEN MILES } \\
\hline NE & 3956 & & & & & 120 & & & 60 & & & & 180 & 120 & & \\
\hline SE & 19858 & & & & & 120 & & & 60 & & & & 180 & 120 & 360 & \\
\hline SW & 1365 & & & & & 120 & & & 60 & & & & 180 & 120 & & \\
\hline NW & 2163 & & & & & 120 & & & 60 & & & & 180 & 120 & & \\
\hline
\end{tabular}


EVACIATION TIME ESTIMATES

Beaver Valley

\begin{tabular}{|c|c|c|c|c|c|c|c|c|c|c|c|c|c|c|c|c|}
\hline 总 & 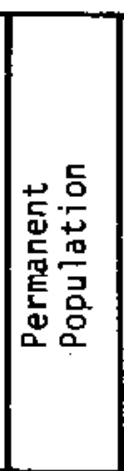 & 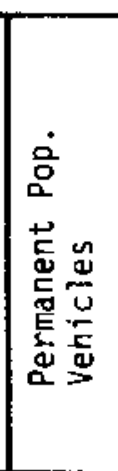 & 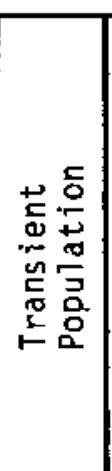 & 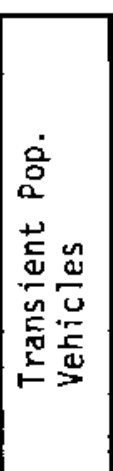 & 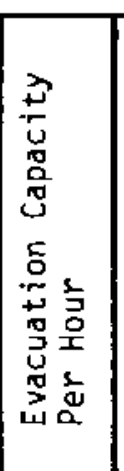 & 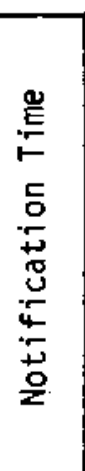 & 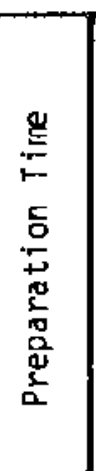 & 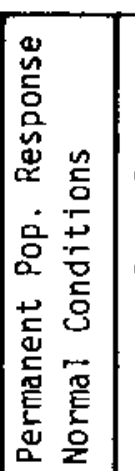 & 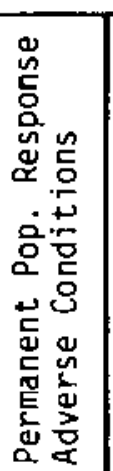 & 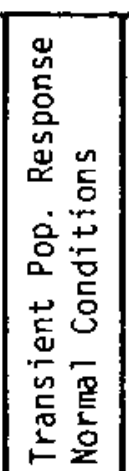 & 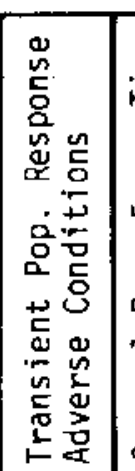 & 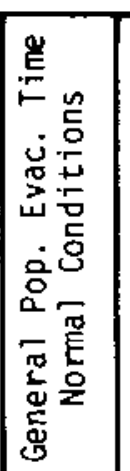 & 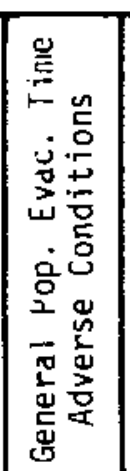 & 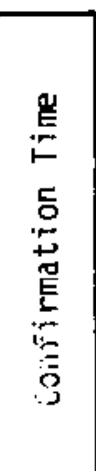 & 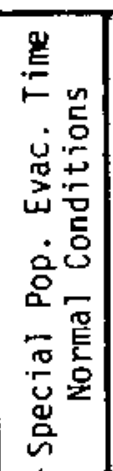 & 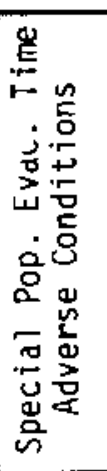 \\
\hline \multicolumn{17}{|c|}{$\frac{1}{\text { WIIHIN TWO MILES }}$} \\
\hline & & & & & & & & & & & & & & & & \\
\hline & & & & & & & & & & & & & & & & \\
\hline \multicolumn{17}{|c|}{ WI THIN FIVE MILES } \\
\hline All & 17300 & & & & & & & & & & & 225 & 270 & & & \\
\hline & & & & & & & & & & & & & & & & \\
\hline & & & & & & & & & & & & & & & & \\
\hline & & & & & & & & & & & & & & & & \\
\hline \multicolumn{17}{|c|}{ WITHIN TEN MILES } \\
\hline A11 & 5559 & & & & & & & & & & & 345 & 435 & & & \\
\hline & & & & & & & & & & & & & & & & \\
\hline & & & & & & & & & & & & & & & & \\
\hline & & & & & & & & & & & & & & & & \\
\hline
\end{tabular}


EVACUATION TIME ESTIMATES

Bjg Rock. Point

\begin{tabular}{|c|c|c|c|c|c|c|c|c|c|c|c|c|c|c|c|c|}
\hline $\begin{array}{l}\text { 峞 } \\
\text { 总 }\end{array}$ & 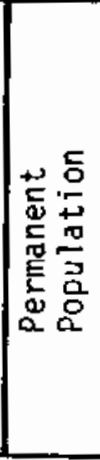 & 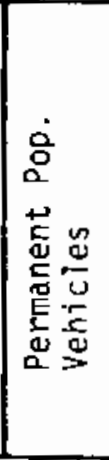 & 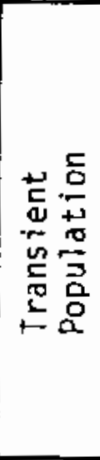 & 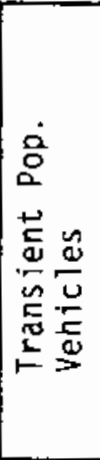 & 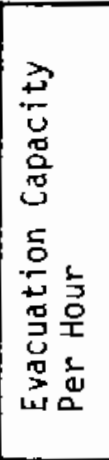 & 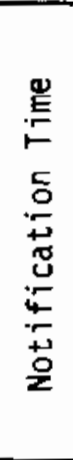 & 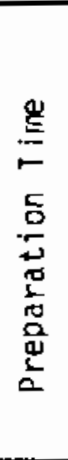 & 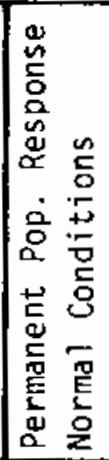 & 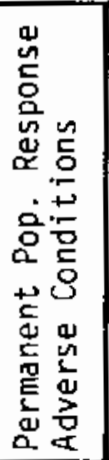 & 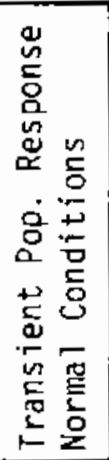 & 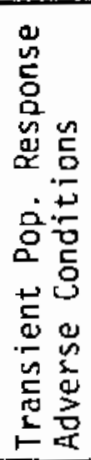 & 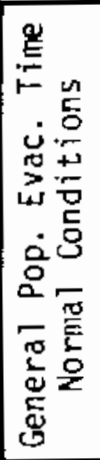 & 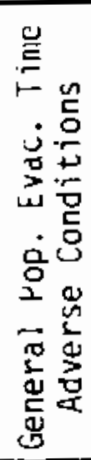 & 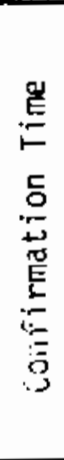 & 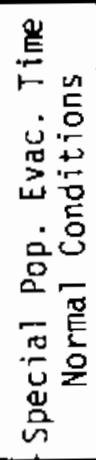 & 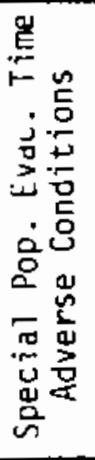 \\
\hline \multicolumn{17}{|c|}{ WITHIN TWO MILES } \\
\hline WSW-NE & & & & & & & & 12 & 14 & & & & & & & \\
\hline \multicolumn{17}{|c|}{ WITHIN FIVE MILES } \\
\hline WSW-S & & & & & & & & 83 & 76 & & & & & & & \\
\hline SSE-NE & & & & & & & & 37 & 26 & & & & & & & \\
\hline & & & & & & & & & & & & & & & & \\
\hline & & & & & & & & & & & & & & & & \\
\hline \multicolumn{17}{|c|}{ WITHIN TEN MILES } \\
\hline WSW-S & & & & & & & & 95 & 75 & & & & & & & \\
\hline SSE-NE & & & & & & & & 71 & 32 & & & & & & & \\
\hline & & & & & & & & & & & & & & & & \\
\hline & & & & & & & & & & & & & & & & \\
\hline
\end{tabular}


EVACUATION TIME ESTIMATES

Browns Ferry

\begin{tabular}{|c|c|c|c|c|c|c|c|c|c|c|c|c|c|c|c|c|}
\hline 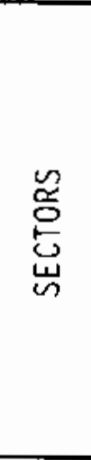 & 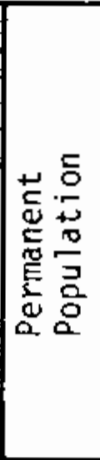 & 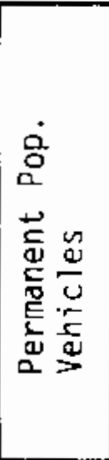 & 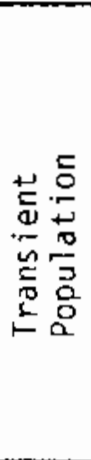 & 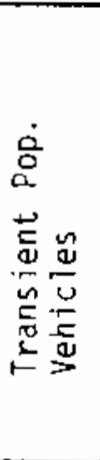 & 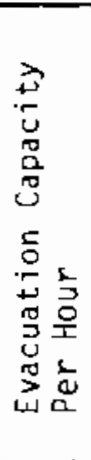 & 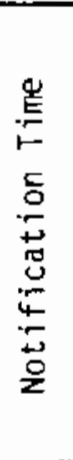 & 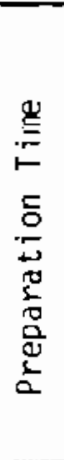 & 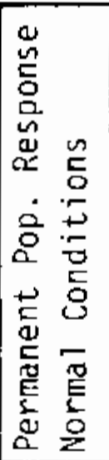 & 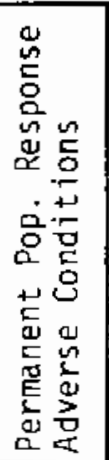 & 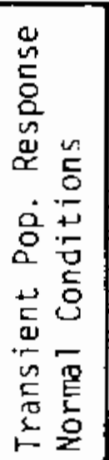 & 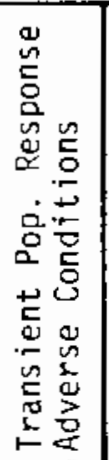 & 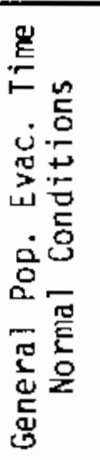 & 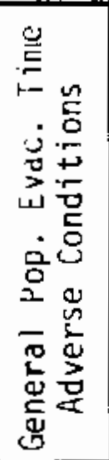 & 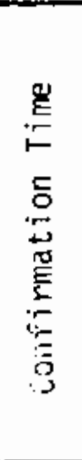 & 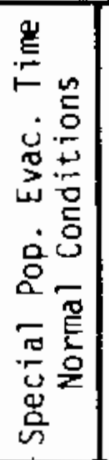 & 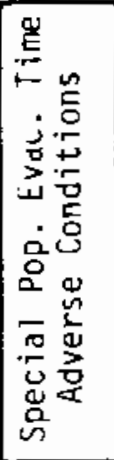 \\
\hline \multicolumn{17}{|c|}{ WITHIN TWO MILES } \\
\hline A11 & & & & & & & & 120 & 240 & & & & & 600 & & \\
\hline & & & & & & & & & & & & & & & & \\
\hline \multicolumn{17}{|c|}{ WITHIN FIVE MILES } \\
\hline Al1 & & & & & & & & 360 & 600 & & & & & 600 & & \\
\hline & & & & & & & & & & & & & & & & \\
\hline & & & & & & & & & & & & & & & & \\
\hline & & & & & & & & & & & & & & & & \\
\hline \multicolumn{17}{|c|}{ WITHIN TEN MILES } \\
\hline All & & & & & & & & 600 & 840 & & & 90 & & 600 & & \\
\hline & & & & & & & & & & & & & & & & \\
\hline & & & & & & & & & & & & & & & & \\
\hline & & & & & & & & & & & & & & & & \\
\hline
\end{tabular}


EVACUATION TIME ESTIMATES

Brunswick*

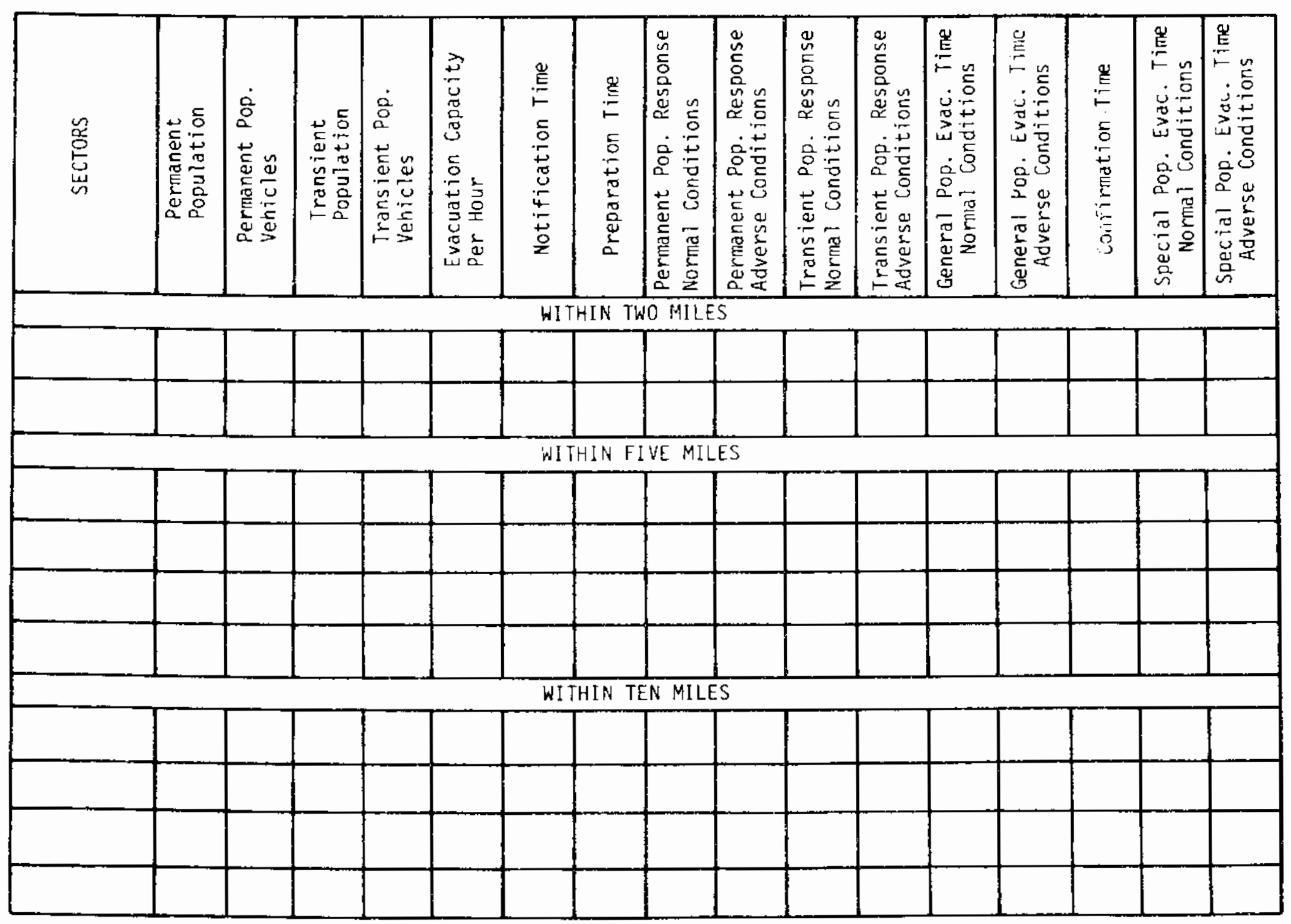

*No Response 
Calvert Cliffs

\begin{tabular}{|c|c|c|c|c|c|c|c|c|c|c|c|c|c|c|c|c|}
\hline $\begin{array}{l}\text { 号 } \\
\text { 它 } \\
\text { 岕 }\end{array}$ & 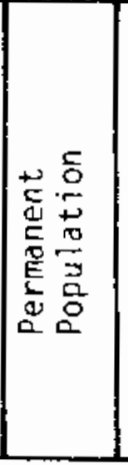 & 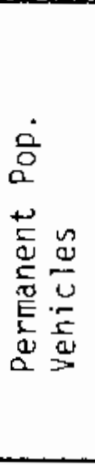 & 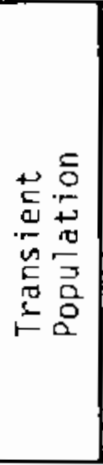 & 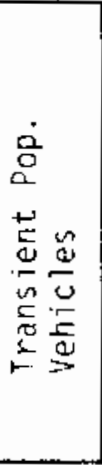 & 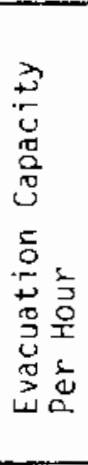 & 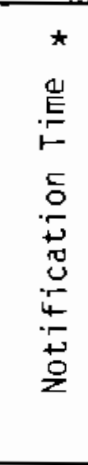 & 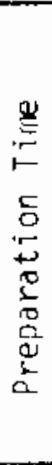 & 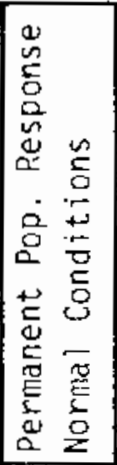 & 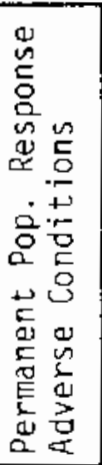 & 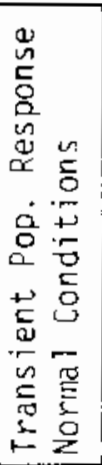 & 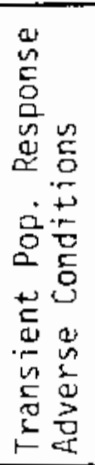 & 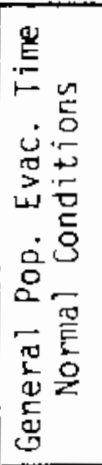 & 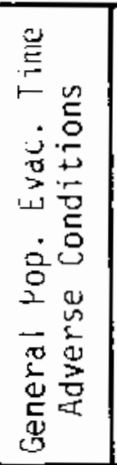 & 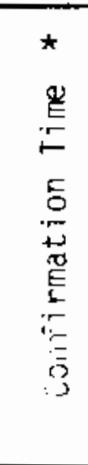 & 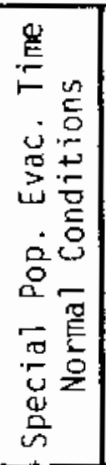 & 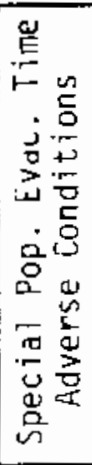 \\
\hline \multicolumn{17}{|c|}{ WITHIN TWO MILES } \\
\hline$N$ & 137 & & & & & $\frac{15}{30}$ & & 273 & 546 & & & 288 & 576 & $\frac{15}{30}$ & & \\
\hline$S$ & 773 & & & & & $\frac{15}{30}$ & & 285 & 570 & & & 300 & 600 & $\frac{15}{30}$ & & \\
\hline \multicolumn{17}{|c|}{ WITHIS FIVE MILES } \\
\hline NW & 2192 & & & & & $\frac{30}{60}$ & & 366 & 722 & & & 396 & 782 & $\frac{30}{60}$ & & \\
\hline SW & 1431 & & & & & $\frac{30}{60}$ & & 348 & 696 & & & 378 & 756 & $\frac{30}{60}$ & & \\
\hline \multicolumn{17}{|l|}{ NE } \\
\hline SE & 934 & & & & & $\frac{30}{60}$ & & 354 & 708 & & & 384 & 768 & $\frac{30}{60}$ & & \\
\hline \multicolumn{17}{|c|}{ WITHIN TEN MILES } \\
\hline $\mathrm{NW}$ & 4903 & & & & & $\frac{60}{20}$ & & 456 & 912 & & & 516 & 1032 & $\frac{60}{120}$ & & \\
\hline SW & 6363 & & & & & $\frac{60}{20}$ & & 486 & 972 & & & 546 & 1096 & $\frac{60}{120}$ & & \\
\hline SE & 10260 & & & & & $\frac{60}{20}$ & & 486 & 972 & & & 546 & 1096 & $\frac{60}{120}$ & & \\
\hline $\mathrm{NE}$ & 601 & & & & & $\frac{60}{20}$ & & 456 & 912 & & & 516 & & $\frac{60}{120}$ & & \\
\hline
\end{tabular}

Notes: * Normal (Top)/Adverse (Bottom) 


\begin{tabular}{|c|c|c|c|c|c|c|c|c|c|c|c|c|c|c|c|c|}
\hline 岕 & 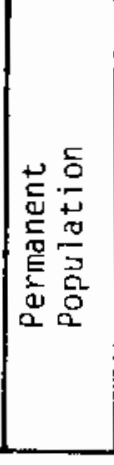 & 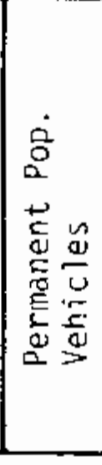 & 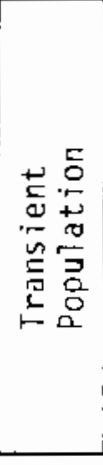 & 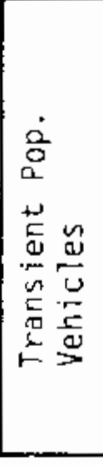 & 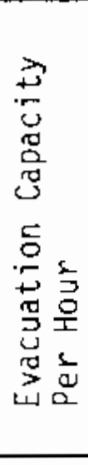 & 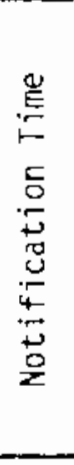 & 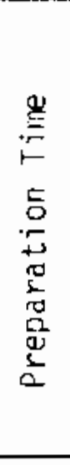 & 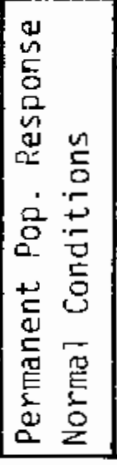 & 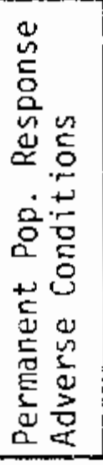 & 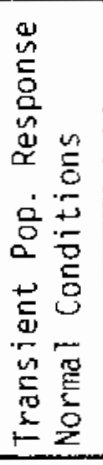 & 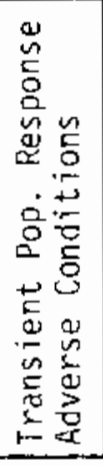 & 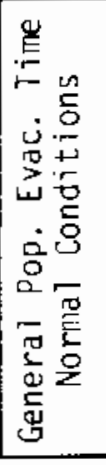 & 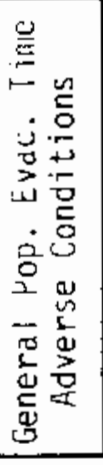 & 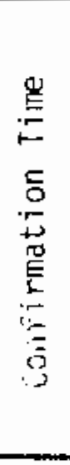 & 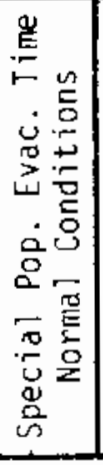 & 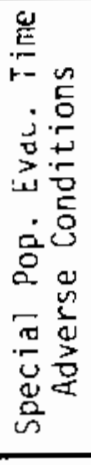 \\
\hline \multicolumn{17}{|c|}{ WITHIN TWO MILES } \\
\hline$A-G$ & 5 & 8 & & & & & & & & & & & & & & \\
\hline$H-R$ & 26 & 8 & & & & & & 7 & & & & & & & & \\
\hline \multicolumn{17}{|c|}{ WITHI IV FIVE MILES } \\
\hline$A-D$ & 308 & 100 & & & & & & & & & & & & & & \\
\hline$E-G$ & 167 & 55 & & & & & & & & & & & & & & \\
\hline$N-R$ & 243 & 80 & & & & & & 29 & & & & & & & & \\
\hline$H-M$ & 363 & 120 & & & & & & 29 & & & & & & & & \\
\hline \multicolumn{17}{|c|}{ WITHIN TEN MILES } \\
\hline$A-D$ & 3045 & 1015 & & & & & & & & & & & & & & \\
\hline$E-G$ & 455 & 150 & 3000 & & & & & & & 210 & & & & & & \\
\hline$N-R$ & 1940 & 632 & & & & 15 & & 64 & & & & & & & & \\
\hline$H-M$ & 1122 & 366 & & & & 15 & & 52 & & & & & & & & \\
\hline
\end{tabular}


Crystal River

\begin{tabular}{|c|c|c|c|c|c|c|c|c|c|c|c|c|c|c|c|c|}
\hline $\begin{array}{l}\text { 岸 } \\
\text { 总 }\end{array}$ & 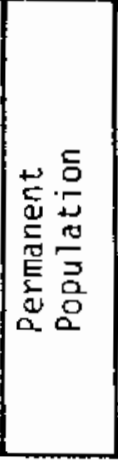 & 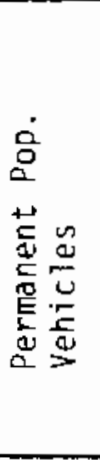 & 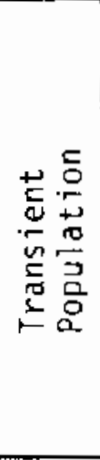 & 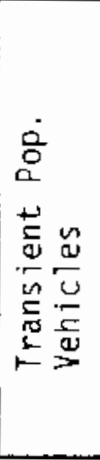 & 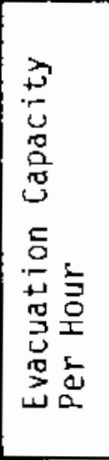 & 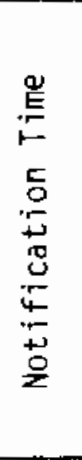 & 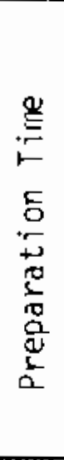 & 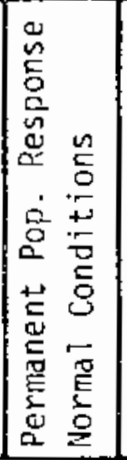 & 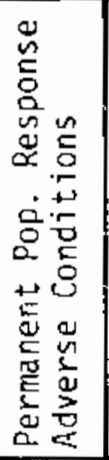 & 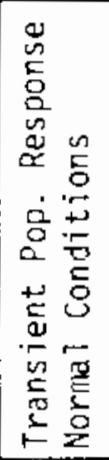 & 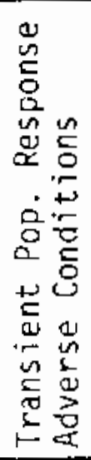 & 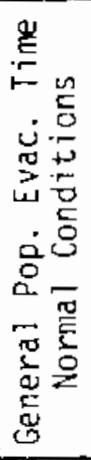 & 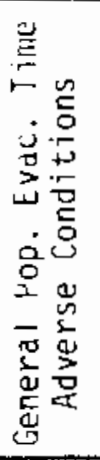 & 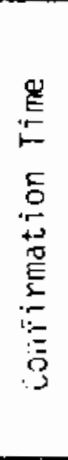 & 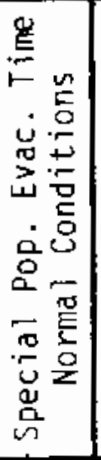 & 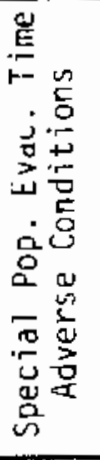 \\
\hline \multicolumn{17}{|c|}{ WITHIN TWO MILES } \\
\hline \multicolumn{17}{|l|}{$A-H$} \\
\hline$J-R$ & 0 & & & & & & & & & & & & & & 60 & 120 \\
\hline \multicolumn{17}{|c|}{ WITHIN FIVE MILES } \\
\hline A-D & 1522 & & & & & 15 & & 150 & 210 & & & 165 & 225 & & & \\
\hline $\mathrm{E}-\mathrm{H}$ & 100 & & & & & 15 & & 45 & 105 & & & 60 & 120 & & & \\
\hline$J-M$ & 0 & & & & & & & & & & & & & & 240 & 360 \\
\hline$N-R$ & 60 & & & & & 15 & & 60 & 120 & & & 75 & 135 & & 240 & 360 \\
\hline \multicolumn{17}{|c|}{ WITHIN TEN MILES } \\
\hline$A-D$ & 2940 & & & & & 15 & & 165 & 345 & & & 180 & 360 & & & \\
\hline$E-H$ & 9000 & & & & & 15 & & 165 & 345 & & & 180 & 360 & & 105 & 180 \\
\hline$J-M$ & 0 & & & & & & & & & & & & & & 360 & 480 \\
\hline$N-R$ & 60 & & & & & 15 & & 60 & 120 & & & 75 & 135 & & 360 & 480 \\
\hline
\end{tabular}




\begin{tabular}{|c|c|c|c|c|c|c|c|c|c|c|c|c|c|c|c|c|}
\hline $\begin{array}{l}\text { 岁 } \\
\text { 总 } \\
\text { 岁 }\end{array}$ & 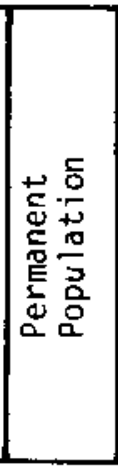 & 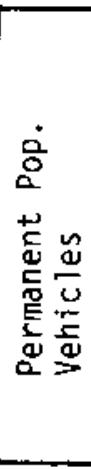 & 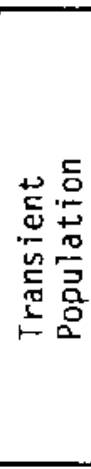 & 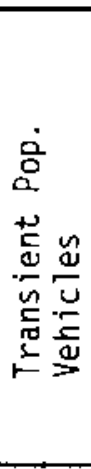 & 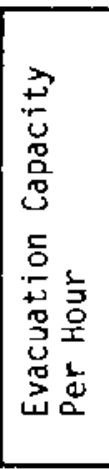 & 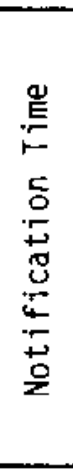 & 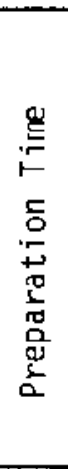 & 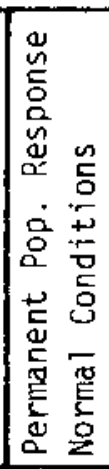 & 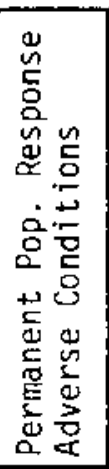 & 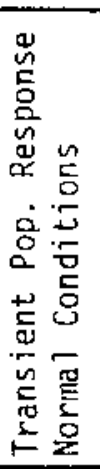 & 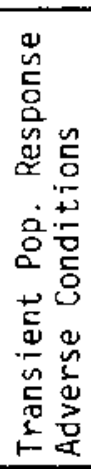 & 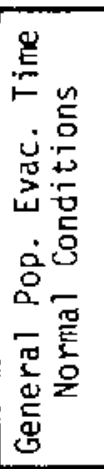 & 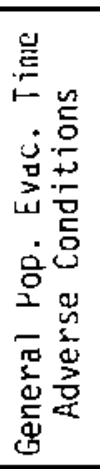 & 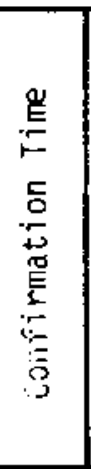 & 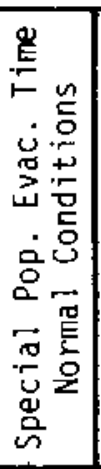 & 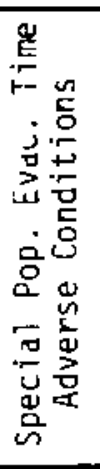 \\
\hline \multicolumn{17}{|c|}{ WITHIN TWO MILES } \\
\hline $180^{\circ}$ & 800 & & & & & & & 40 & 80 & & & & & 120 & & \\
\hline \multirow{2}{*}{\multicolumn{17}{|c|}{ WITHIN FIVE MILES }} \\
\hline & & & & & & & & & & & & & & & & \\
\hline Left & 1600 & & & & & & & 70 & 140 & & & & & 120 & & \\
\hline Right & 1800 & & & & & & & 75 & 150 & & & & & 120 & & \\
\hline & & & & & & & & & & & & & & & & \\
\hline & & & & & & & & & & & & & & & & \\
\hline \multicolumn{17}{|c|}{ WITHIN TEN MILES } \\
\hline Left & 6600 & & & & & & & 120 & 240 & & & & & 120 & & \\
\hline Right & 11800 & & & & & & & 210 & 420 & & & & & 120 & & \\
\hline & & & & & & & & & & & & & & & & \\
\hline & & & & & & & & & & & & & & & & \\
\hline
\end{tabular}


D. C. Cook

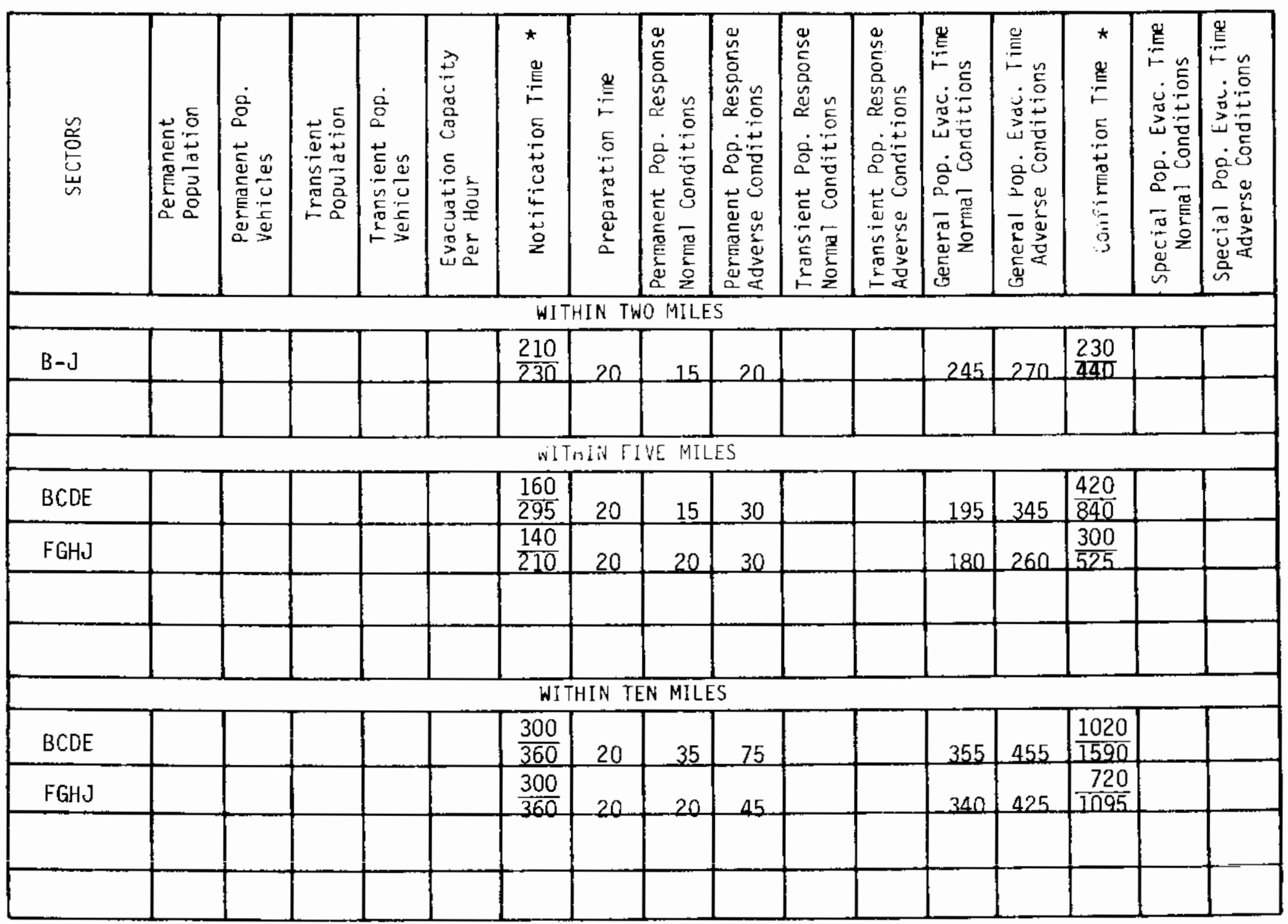

Note: * Normal (Top)/Adverse (Bottom) 
Diablo Canyon

\begin{tabular}{|c|c|c|c|c|c|c|c|c|c|c|c|c|c|c|c|c|}
\hline 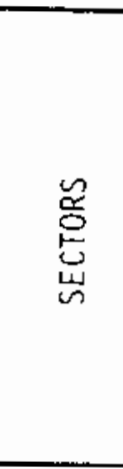 & 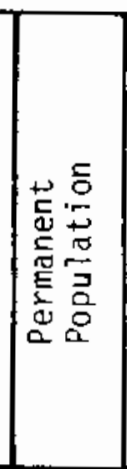 & 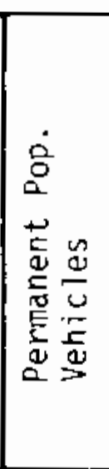 & 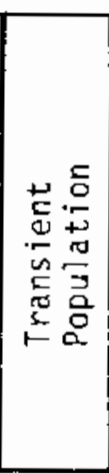 & 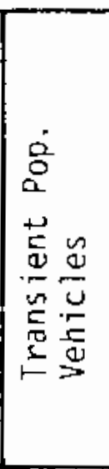 & 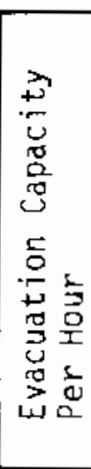 & 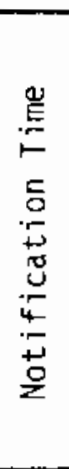 & 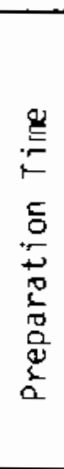 & 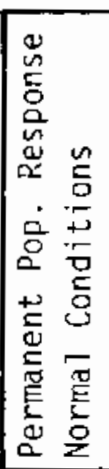 & 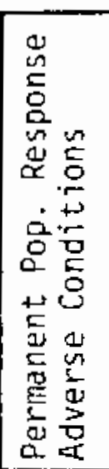 & 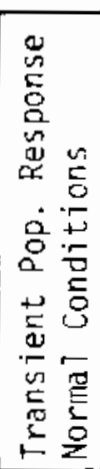 & 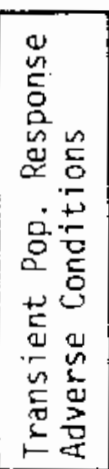 & 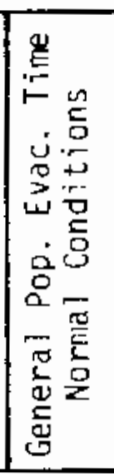 & 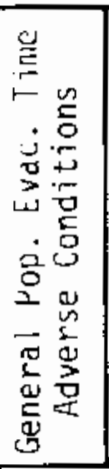 & 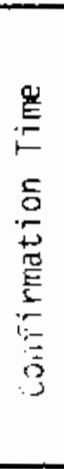 & 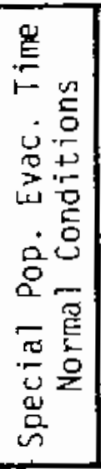 & 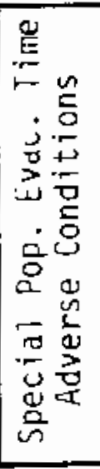 \\
\hline \multicolumn{17}{|c|}{ WITHIN TWO MILES } \\
\hline \multicolumn{17}{|c|}{ QRA-F } \\
\hline & & & & & & & & & & & & & & & & \\
\hline \multicolumn{17}{|c|}{ WITHII IN FIVE MILES } \\
\hline \multicolumn{17}{|l|}{ RAB } \\
\hline \multicolumn{17}{|l|}{$\mathrm{COE}$} \\
\hline \multicolumn{17}{|l|}{ EF } \\
\hline & & & & & & & & & & & & & & & & \\
\hline \multicolumn{17}{|c|}{ WITHIN TEN MILES } \\
\hline RAB & 11763 & & & & & & & & & & & & & & & \\
\hline $\mathrm{COE}$ & 4965 & & & & & & & & & & & & & & & \\
\hline$E F$ & 917 & & & & & & & & & & & & & & & \\
\hline ALL & 63489 & 29785 & & & & & & & & & & 255 & 300 & & & \\
\hline
\end{tabular}

Note: This study siected to use a 6 -mile rather than a 5 -mile radius and 67.5 degree sectors to better reflect natural boundaries. The 10-mile radius was expanded up to 12 miles so as not to divide population centers. 


\begin{tabular}{|c|c|c|c|c|c|c|c|c|c|c|c|c|c|c|c|c|}
\hline 岕 & 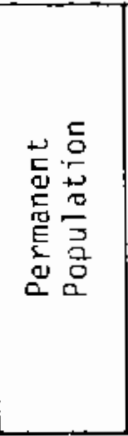 & 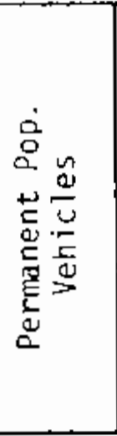 & 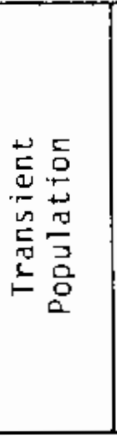 & 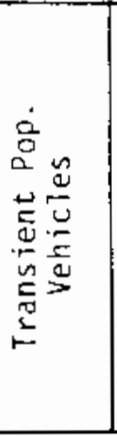 & 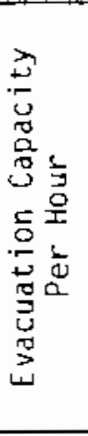 & 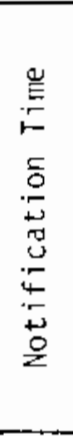 & 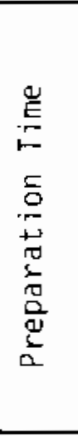 & 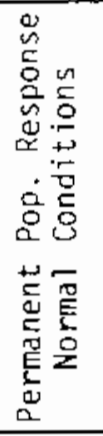 & 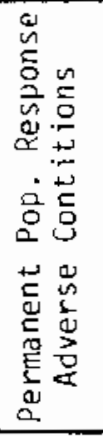 & 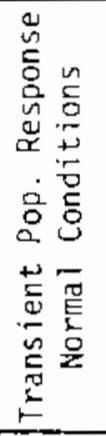 & 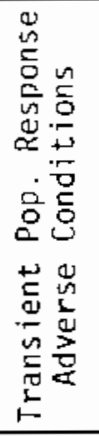 & 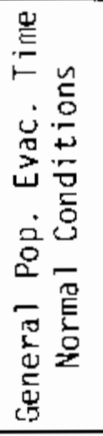 & 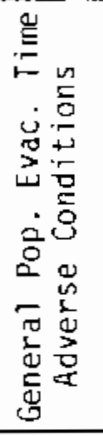 & 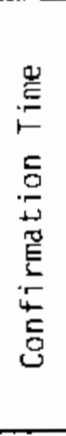 & 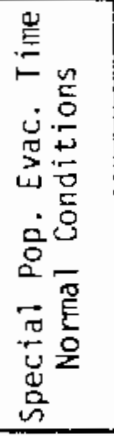 & 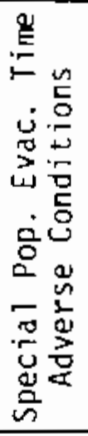 \\
\hline \multicolumn{17}{|c|}{ WITHIN TWO MILES } \\
\hline I & 80 & 30 & 830 & 660 & & 45 & 20 & & & & & 240 & & & & \\
\hline II & 580 & 200 & 980 & 750 & & 45 & 20 & & & & & 360 & & & & \\
\hline & & & & & & & & & & & & & & & & \\
\hline & & & & & & & & & & & & & & & & \\
\hline & & & & & & & & & & & & & & & & \\
\hline \multicolumn{17}{|c|}{ WITHIN FIVE MILES } \\
\hline I, III & 4280 & 1430 & 1280 & 960 & & 45 & 20 & & & & & 300 & & & & \\
\hline$I, V I I$ & 760 & 255 & 1010 & 810 & & 45 & 20 & & & & & 480 & & & 60 & \\
\hline II, IV & 1840 & 615 & 980 & 755 & & 60 & 20 & & & & & 420 & & & 60 & \\
\hline II,$V$ & 710 & 240 & 980 & 755 & & 60 & 20 & & & & & 480 & & & & \\
\hline$I I, V I$ & 1690 & 570 & 980 & 750 & & 45 & 20 & & & & & 480 & & & & \\
\hline \multicolumn{17}{|c|}{ WITHIN TEN MILES } \\
\hline$I$, III, VIII & 12340 & 4110 & 1580 & 1210 & & 45 & 20 & & & & & 360 & & & 60 & \\
\hline$I, V I I, X I I$ & 10840 & 3560 & 1010 & 865 & & 45 & 20 & & & & & 360 & & & 120 & \\
\hline$I I, I V, I X$ & 9410 & 3140 & 1280 & 995 & & 60 & 20 & & & & & 420 & & & 180 & \\
\hline$I I, V, X$ & 3400 & 1135 & 980 & 755 & & 60 & 20 & & & & & 480 & & & 60 & \\
\hline $\bar{I} \bar{I}, V \bar{I}, X \bar{I}$ & 6870 & 2290 & 1050 & 810 & & 45 & 20 & & & & & 480 & & & 60 & \\
\hline
\end{tabular}

Note: Evacuation times shown are in some cases less for 10 mile radius than for evacuation of a smaller part of the same sectors. This is not reasonable, although possible from methodology used. 
Duane Arnold

\begin{tabular}{|c|c|c|c|c|c|c|c|c|c|c|c|c|c|c|c|c|}
\hline $\begin{array}{l}\mathscr{2} \\
\text { 总 } \\
\text { 岁 }\end{array}$ & 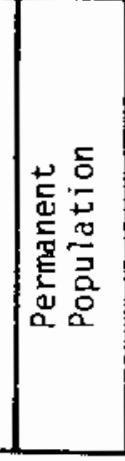 & 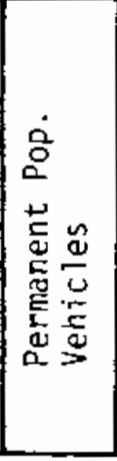 & 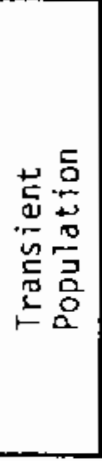 & 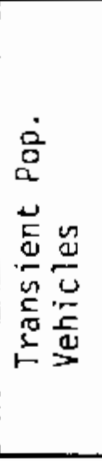 & 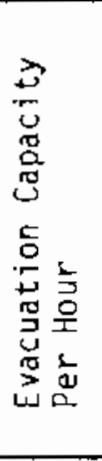 & 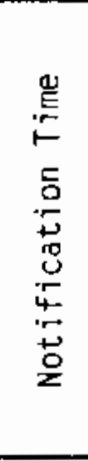 & 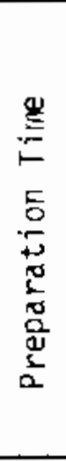 & 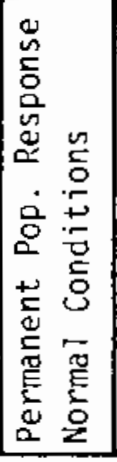 & 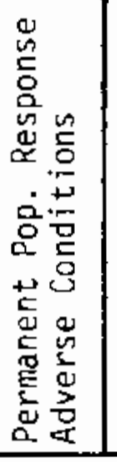 & 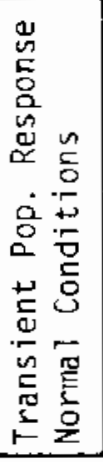 & 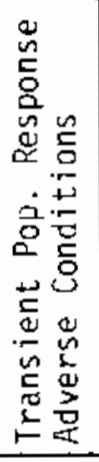 & 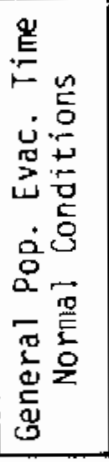 & 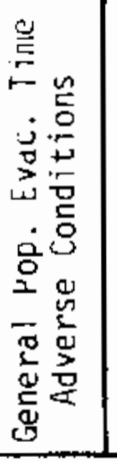 & 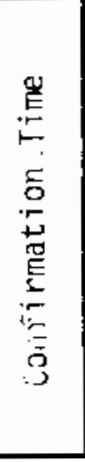 & 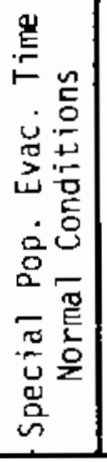 & 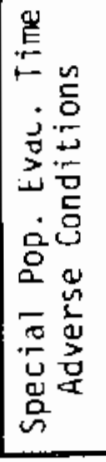 \\
\hline \multicolumn{17}{|c|}{ WITHIN TWO MILES } \\
\hline $\mathrm{NW}$ & 129 & 43 & & & & 10 & & 45 & 106 & & & 55 & 116 & 60 & & \\
\hline SE & 198 & 66 & & & & 10 & & 42 & 96 & & & 52 & 106 & 60 & & \\
\hline \multicolumn{17}{|c|}{ 'AITHIN FIVE MILES } \\
\hline NW & 522 & 174 & & & & 30 & & 58 & 119 & & & 88 & 149 & 60 & & \\
\hline NE & 777 & 259 & & & & 40 & & 56 & 110 & & & 96 & 150 & 60 & & \\
\hline SE & 1002 & 334 & & & & 51 & & 69 & 123 & & & 120 & 174 & 60 & & \\
\hline SW & 1323 & 441 & & & & 70 & & 61 & 115 & & & 131 & 185 & 60 & & \\
\hline \multicolumn{17}{|c|}{ WITHIN TEN MILES } \\
\hline NW & 4473 & 1491 & & & & 160 & & 138 & 192 & & & 298 & 352 & 120 & & \\
\hline $\mathrm{NE}$ & 2649 & 883 & & & & 107 & & 84 & 138 & & & 191 & 245 & 120 & & \\
\hline SE & $\$ 1002$ & 50334 & & & & 231 & & 402 & 420 & & & 6.33 & 651 & 120 & 165 & 192 \\
\hline SW & 3525 & 1175 & & & & 149 & & 90 & 144 & & & 239 & 293 & 120 & & \\
\hline
\end{tabular}




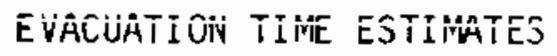

Farley

\begin{tabular}{|c|c|c|c|c|c|c|c|c|c|c|c|c|c|c|c|c|}
\hline $\begin{array}{l}\text { Oै } \\
\text { 总 } \\
\text { 出 }\end{array}$ & 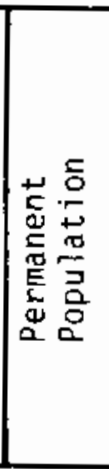 & 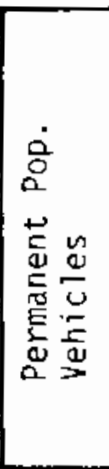 & 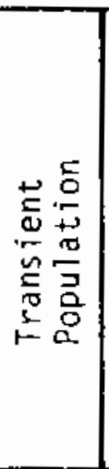 & 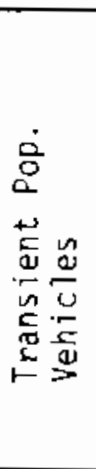 & 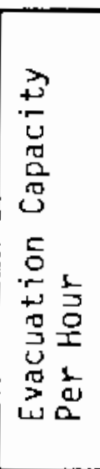 & 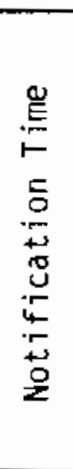 & 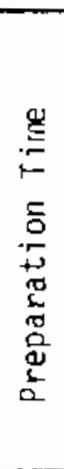 & 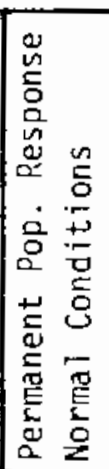 & 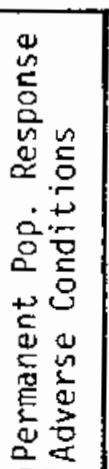 & 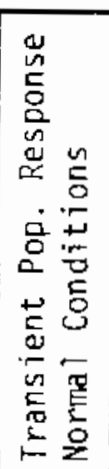 & 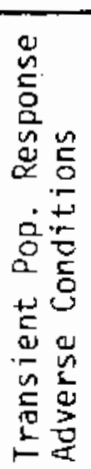 & 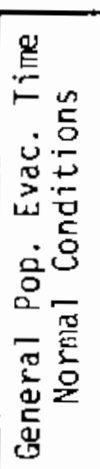 & 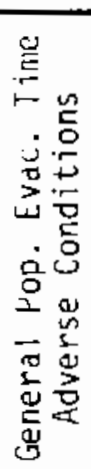 & 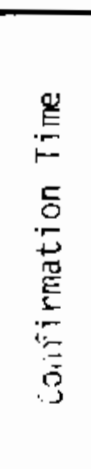 & 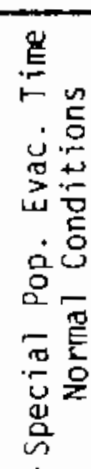 & 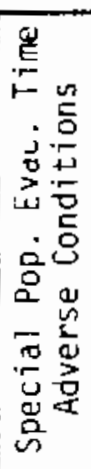 \\
\hline \multicolumn{17}{|c|}{ WITHIN TWO MILES } \\
\hline$E$ & & & & & & & & 45 & 60 & & & & & 30 & 0 & 0 \\
\hline$W$ & & & & & & & & 45 & 60 & & & & & 30 & 0 & 0 \\
\hline \multicolumn{17}{|c|}{ WITHI N FIVE MILES } \\
\hline NE & & & & & & & & 120 & 180 & & & & & 60 & 0 & 0 \\
\hline SE & & & & & & & & 120 & 180 & & & & & 60 & 0 & 0 \\
\hline SW & & & & & & & & 120 & 180 & & & & & 60 & 0 & 0 \\
\hline $\mathrm{NW}$ & & & & & & & & 120 & 180 & & & & & 60 & 0 & 0 \\
\hline \multicolumn{17}{|c|}{ WITHIN TEN MILES } \\
\hline NE & & & & & & & & 240 & 360 & & & & & 90 & 0 & 0 \\
\hline SE & & & & & & & & 360 & 480 & & & & & 120 & 0 & 0 \\
\hline SW & & & & & & & & 360 & 480 & & & & & 150 & 0 & 0 \\
\hline NW & & & & & & & & 360 & 480 & & & & & 150 & $\overline{0}$ & $\hat{0}$ \\
\hline
\end{tabular}


EVACUATION TIME ESTIMATES

Fitzpatrick*

\begin{tabular}{|c|c|c|c|c|c|c|c|c|c|c|c|c|c|c|c|c|}
\hline 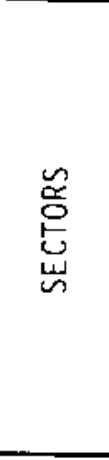 & 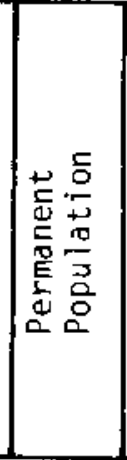 & 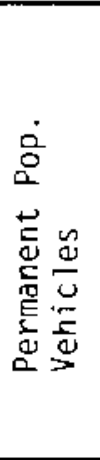 & 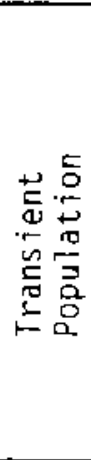 & 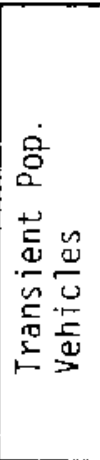 & 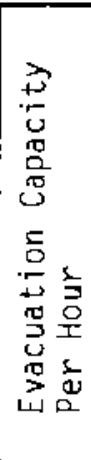 & 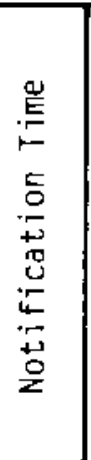 & 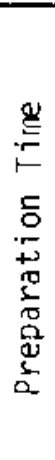 & 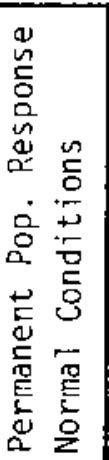 & 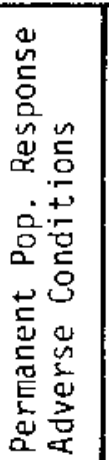 & 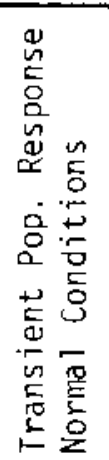 & 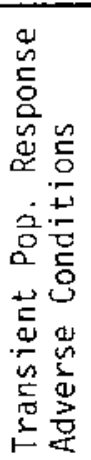 & 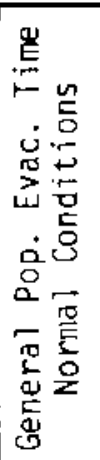 & 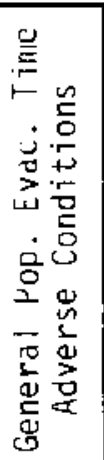 & 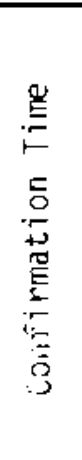 & 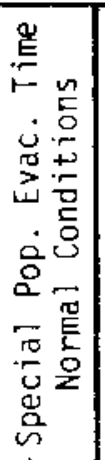 & 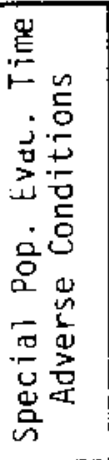 \\
\hline \multicolumn{17}{|c|}{ WITHIN TWO MILES } \\
\hline 5 & 1088 & & & & & 60 & & 70 & 90 & & & 130 & 150 & 90 & & \\
\hline \multicolumn{17}{|c|}{ WITHISN FIVE MILES } \\
\hline SW & 3733 & & & & & 60 & & 70 & 100 & & & 130 & 160 & 150 & & \\
\hline SE & 2824 & & & & & 60 & & 70 & 100 & & & 130 & 160 & 150 & & \\
\hline & & & & & & & & & & & & & & & & \\
\hline & & & & & & & & & & & & & & & & \\
\hline \multicolumn{17}{|c|}{ WI THIN TEN MILES } \\
\hline SW & 35973 & & & & & 60 & & 170 & 280 & & & 230 & 340 & 210 & 130 & 210 \\
\hline SE & 9575 & & & & & 60 & & 90 & 120 & & & 150 & 180 & 210 & & \\
\hline Lake & & & & & & & & & & & & & & & 45 & \\
\hline Al 1 & 43772 & & & & & 60 & & 170 & 280 & & & 230 & 340 & 360 & 130 & 210 \\
\hline
\end{tabular}

* Same location as Nine Mile Point. 
Ft. Calhoun

\begin{tabular}{|c|c|c|c|c|c|c|c|c|c|c|c|c|c|c|c|c|}
\hline $\begin{array}{l}\text { 苍 } \\
\text { 总 } \\
\text { S }\end{array}$ & 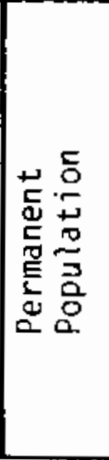 & 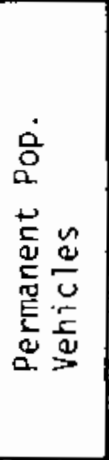 & 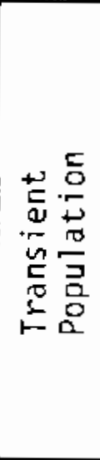 & 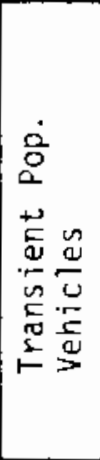 & 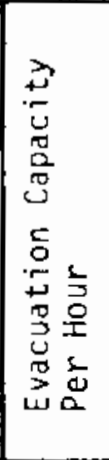 & 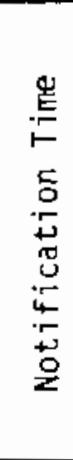 & 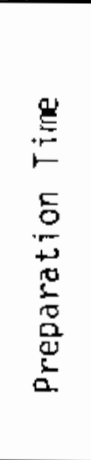 & 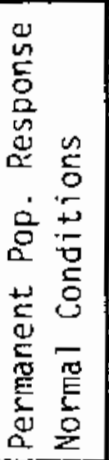 & 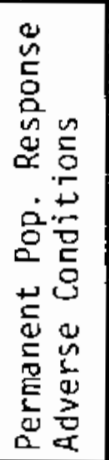 & 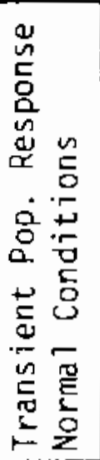 & 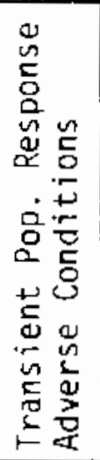 & 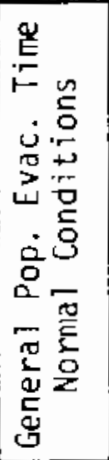 & 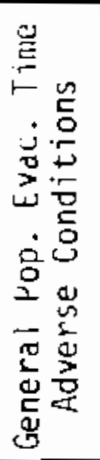 & 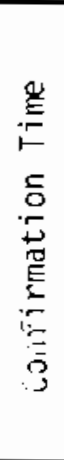 & 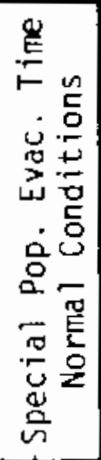 & 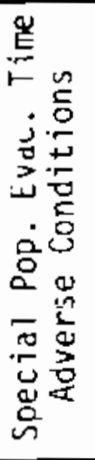 \\
\hline \multicolumn{17}{|c|}{ WI THIN TWO MILES } \\
\hline$G-R$ & 187 & 62 & & & & 15 & 180 & 10 & & & & 205 & & & & \\
\hline Iowa & & & & & & & & & & & & & & & & \\
\hline \multicolumn{17}{|c|}{ WITHIN FIVE MILES } \\
\hline $\mathrm{N}-\mathrm{R}$ & 7382 & 2077 & & & & 15 & 180 & 42 & & & & 237 & & & & \\
\hline G-M & 892 & 274 & & & & 15 & 180 & 20 & & & & 215 & & & & \\
\hline \multicolumn{17}{|l|}{ Iowa } \\
\hline \multicolumn{17}{|c|}{ WITHIN TEN MILES } \\
\hline $\mathrm{N}-\mathrm{R}$ & 8317 & & & & & 15 & 180 & 56 & & & & 251 & & & & \\
\hline$G-M$ & 2374 & & & & & 15 & 180 & 40 & & & & 235 & & & & \\
\hline Iowa & & & & & & 15 & 90 & 20 & 40 & & & 125 & 145 & & & \\
\hline & & & & & & & & & & & & & & & & \\
\hline
\end{tabular}


Ft. St. Vrain

\begin{tabular}{|c|c|c|c|c|c|c|c|c|c|c|c|c|c|c|c|c|}
\hline 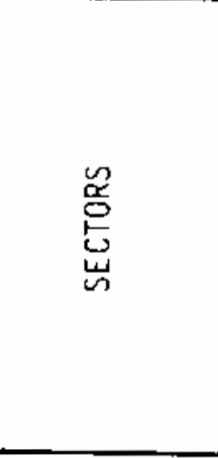 & 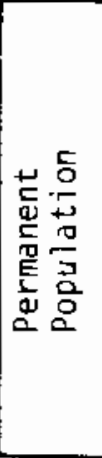 & 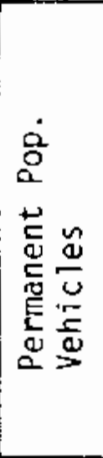 & 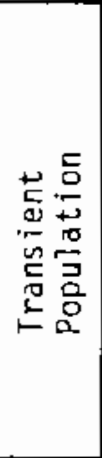 & 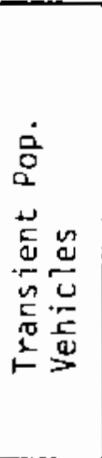 & 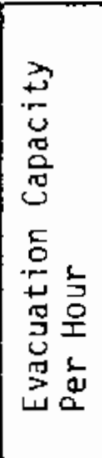 & 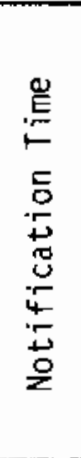 & 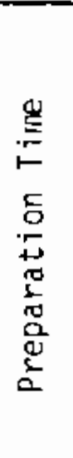 & 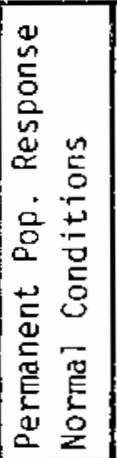 & 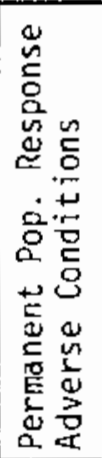 & 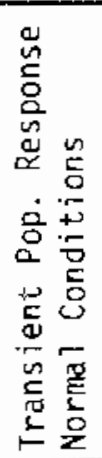 & 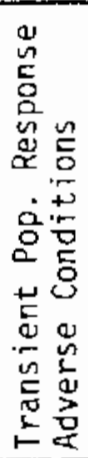 & 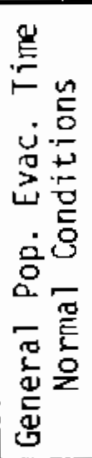 & 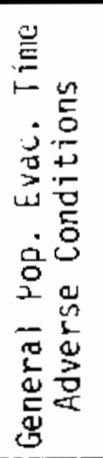 & 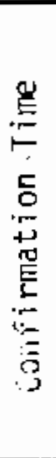 & 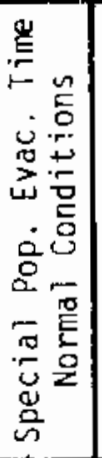 & 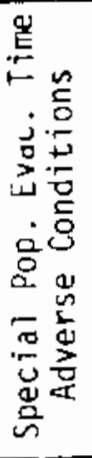 \\
\hline \multicolumn{17}{|c|}{ WITHIN TWO MILES } \\
\hline I & 140 & 64 & 350 & 315 & 5000 & 126 & 20 & 94 & & & & 240 & & & & \\
\hline II & 76 & 35 & & & 3000 & 36 & 20 & 64 & & & & 120 & & & & \\
\hline \multicolumn{17}{|c|}{ WITHIN FIVE MILES } \\
\hline$I \& V$ & 554 & 252 & 350 & 315 & 4000 & 360 & 20 & 40 & & & & 420 & & & & \\
\hline$I \& V I$ & 744 & 338 & 350 & 315 & 4000 & 180 & 20 & 280 & & & & 480 & & & & \\
\hline II \& III & 374 & 170 & & & 3000 & 150 & 20 & 130 & & & & 300 & & & & \\
\hline II \& IV & 1802 & 819 & & & 1500 & 144 & 20 & 316 & & & & 480 & & & & \\
\hline \multicolumn{17}{|c|}{ WITHIN TEN MILES } \\
\hline$I, V, I X$ & 4099 & 1863 & 350 & 315 & 7000 & 336 & 20 & 184 & & & & 540 & & & 60 & \\
\hline$I, V I, X$ & 5121 & 2328 & 350 & 315 & 7000 & 246 & 20 & 274 & & & & 540 & & & 18 & \\
\hline II, II I , VII & 1736 & 774 & & & 3500 & 282 & 20 & 238 & & & & 540 & & & 18 & \\
\hline I I , IV , VI I I & 2244 & 1020 & & & 3500 & 246 & 20 & 334 & & & & 600 & & & 18 & \\
\hline
\end{tabular}




\section{Ginna}

\begin{tabular}{|c|c|c|c|c|c|c|c|c|c|c|c|c|c|c|c|c|}
\hline 号 & 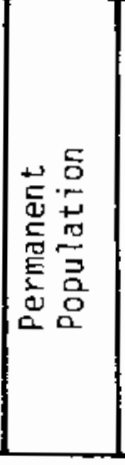 & 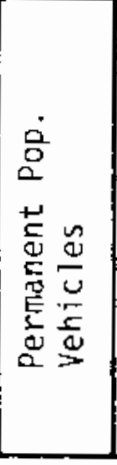 & 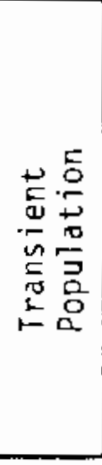 & 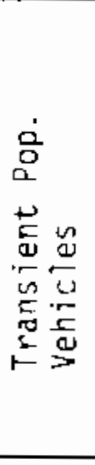 & 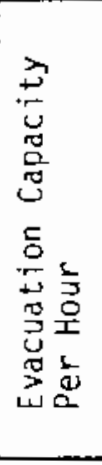 & 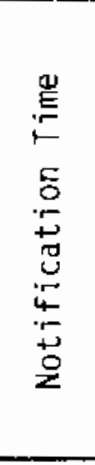 & 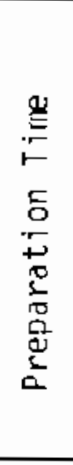 & 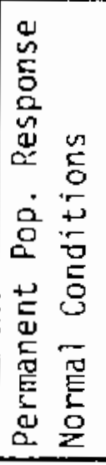 & 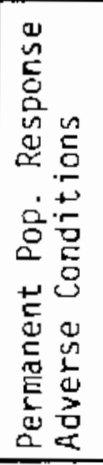 & 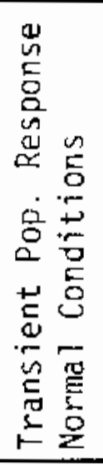 & 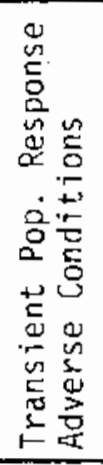 & 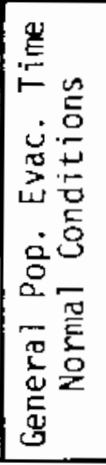 & 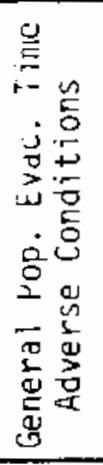 & 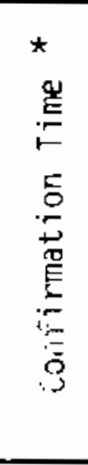 & 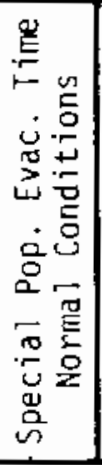 & 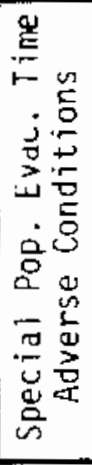 \\
\hline \multicolumn{17}{|c|}{ WITHIN TWO MILES } \\
\hline 5 & 867 & 237 & & & & 120 & 20 & 39 & 60 & & & & & $\frac{45}{90}$ & & \\
\hline N & & & & & & & & & & 30 & 45 & & & $\frac{60}{90}$ & 270 & 285 \\
\hline \multicolumn{17}{|c|}{ WITHIN FIVE MILES } \\
\hline SE & 4820 & 1197 & & & & 120 & 20 & 92 & 117 & & & & & $\frac{60}{120}$ & & \\
\hline SW & 4820 & 1198 & & & & 120 & 20 & 92 & 117 & & & & & $\frac{180}{360}$ & 120 & \\
\hline $\mathrm{NW}$ & 0 & & & & & & & & & & & & & $\frac{75}{113}$ & 390 & 420 \\
\hline NE & 0 & & & & & & & & & & & & & $\frac{75}{113}$ & 390 & 420 \\
\hline \multicolumn{17}{|c|}{ WITHIN TEN MILES } \\
\hline SE & 12064 & 3414 & & & & 120 & 20 & 114 & 236 & & & & & $\frac{135}{270}$ & & \\
\hline SW & 36803 & 10868 & & & & 120 & 20 & 312 & 384 & & & & & $\frac{360}{720}$ & & \\
\hline NW & 0 & & & & & & & & & & & & & $\frac{105}{158}$ & 550 & 595 \\
\hline NE & 0 & & & & & & & & & & & & & $\frac{105}{158}$ & 550 & 595 \\
\hline
\end{tabular}

Note: All north sectors are Lake Ontario, and time estimates for lake sectors are for evacuating boaters. Evacuation time shown under Special Population.

* Normal (Top)/Adverse (Bottom) 


\begin{tabular}{|c|c|c|c|c|c|c|c|c|c|c|c|c|c|c|c|c|}
\hline $\begin{array}{l}\text { 哣 } \\
\text { 出 }\end{array}$ & 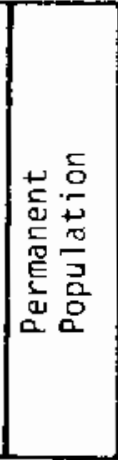 & 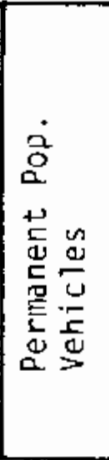 & 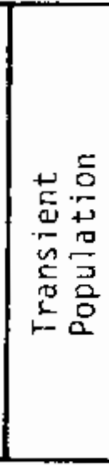 & 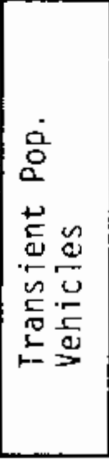 & 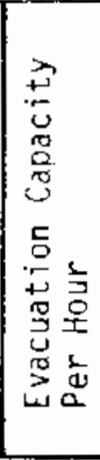 & 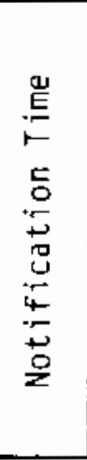 & 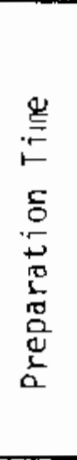 & 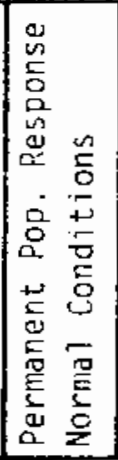 & 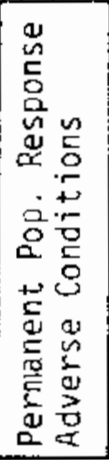 & 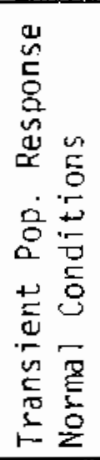 & 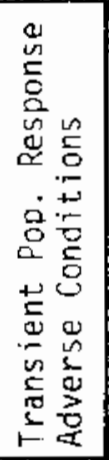 & 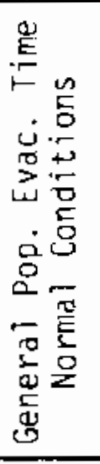 & 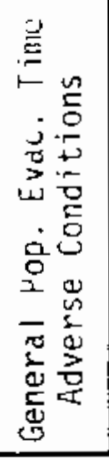 & 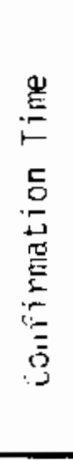 & 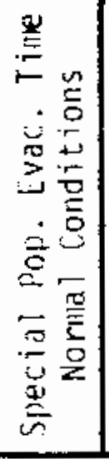 & 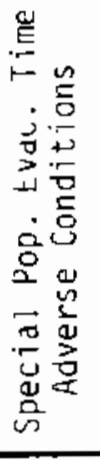 \\
\hline \multicolumn{17}{|c|}{ WITHIN TWO MILES } \\
\hline$A B$ & 1304 & 869 & 0 & 0 & 2040 & 20 & & & & & & 67 & 79 & & & \\
\hline$C D$ & 1616 & 1077 & 407 & 271 & 2325 & 22 & & & & & & 73 & 96 & & & \\
\hline \multicolumn{17}{|c|}{ WI THIN FIVE MILES } \\
\hline A & 3540 & 2361 & 4317 & 2878 & 3489 & 57 & & & & & & 178 & 209 & & & \\
\hline B & 4025 & 2683 & 0 & 0 & 1571 & 59 & & & & & & 165 & 192 & & & \\
\hline C & 2636 & 1757 & 0 & 0 & 2623 & 65 & & & & & & 124 & 139 & & & \\
\hline D & 2635 & 1757 & 1872 & 1248 & 6290 & 65 & & & & & & 96 & 109 & & & \\
\hline \multicolumn{17}{|c|}{ WITHIN TEN MILES } \\
\hline A & 38316 & 25544 & 5992 & 3995 & 6169 & 175 & & & & & & 477 & 593 & & 240 & 300 \\
\hline B & 7982 & 5321 & 7503 & 5002 & 3877 & 325 & & & & & & 486 & 527 & & & \\
\hline$c$ & 15890 & 10593 & 10866 & 7444 & 7384 & 260 & & & & & & 495 & 354 & & & \\
\hline$D$ & 10326 & 6884 & 5054 & 3369 & 8791 & 208 & & & & & & 281 & 307 & & & \\
\hline
\end{tabular}




\begin{tabular}{|c|c|c|c|c|c|c|c|c|c|c|c|c|c|c|c|c|}
\hline 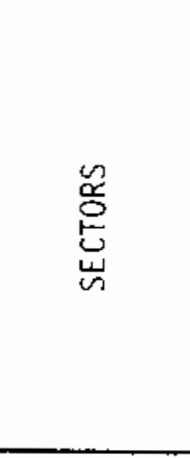 & 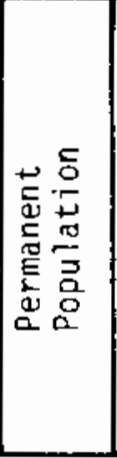 & 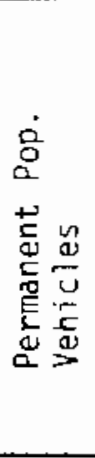 & 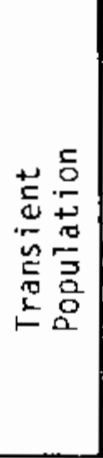 & 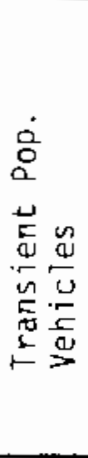 & 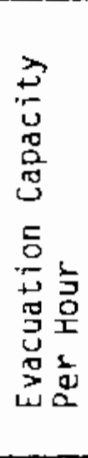 & 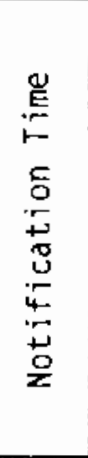 & 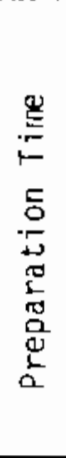 & 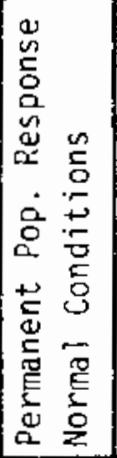 & 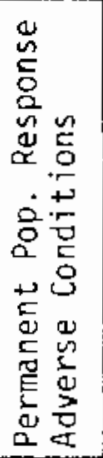 & 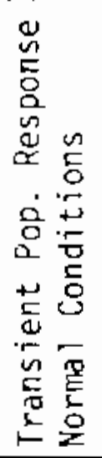 & 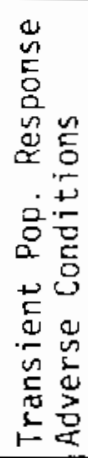 & 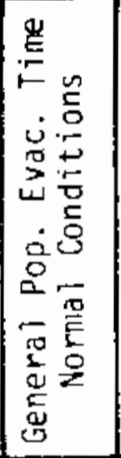 & 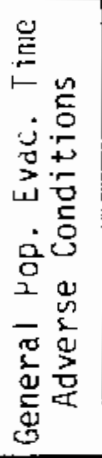 & 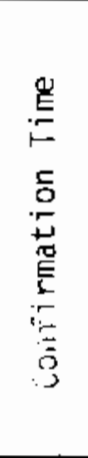 & 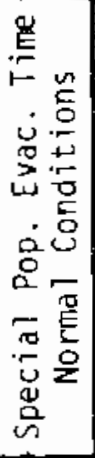 & 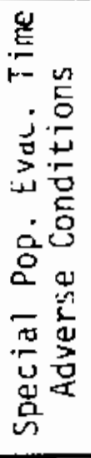 \\
\hline \multicolumn{17}{|c|}{ WITHIN TWO MILES } \\
\hline$E-M$ & 69 & & & & & 30 & & 30 & 90 & & & 60 & 120 & 30 & & \\
\hline$A-E, M-P$ & 266 & & & & & 30 & & 30 & 30 & & & 60 & 60 & 30 & & \\
\hline \multicolumn{17}{|c|}{ dITHlin FIVE MILES } \\
\hline$E-M$ & 545 & & & & & 180 & & 90 & 180 & & & 270 & 360 & 180 & & \\
\hline$A-E, M-P$ & 616 & & & & & 45 & & 90 & 120 & & & 135 & 165 & 30 & & \\
\hline & & & & & & & & & & & & & & & & \\
\hline & & & & & & & & & & & & & & & & \\
\hline \multicolumn{17}{|c|}{ WI THIN TEN MILES } \\
\hline$E-M$ & 2986 & & & & & 360 & & 180 & 360 & & & 540 & 720 & 360 & & \\
\hline$A-E, M-P$ & 3412 & & & & & 240 & & 360 & 450 & & & 600 & 690 & 240 & & \\
\hline & & & & & & & & & & & & & & & & \\
\hline & & & & & & & & & & & & & & & & \\
\hline
\end{tabular}


Indian Point

\begin{tabular}{|c|c|c|c|c|c|c|c|c|c|c|c|c|c|c|c|c|}
\hline $\begin{array}{l}\text { 号 } \\
\text { 总 } \\
\text { S }\end{array}$ & 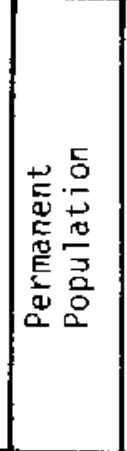 & 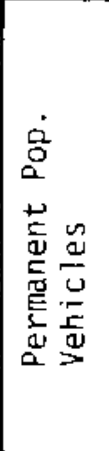 & 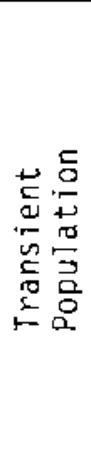 & 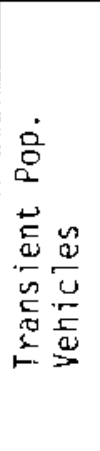 & 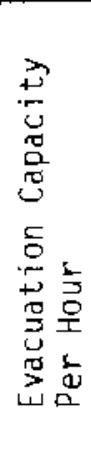 & 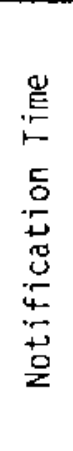 & 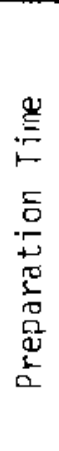 & 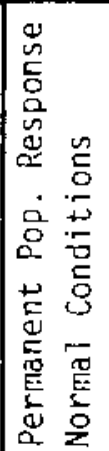 & 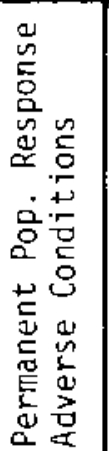 & 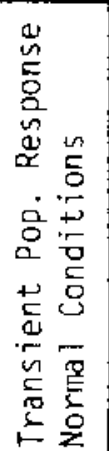 & 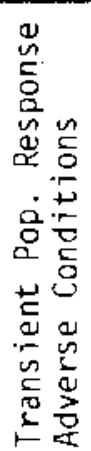 & 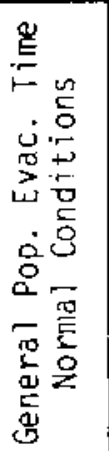 & 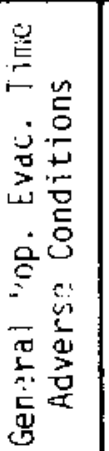 & 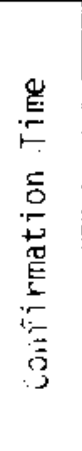 & 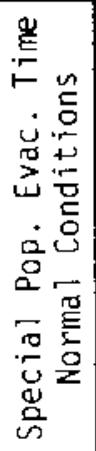 & 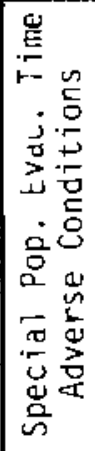 \\
\hline \multicolumn{17}{|c|}{ WI THIN TWO MILES } \\
\hline 1 & 32112 & & & & & 60 & & 200 & 330 & & & 260 & 390 & & 430 & 610 \\
\hline 2 & 12191 & & & & & 90 & & 200 & 330 & & & 290 & 420 & 120 & 430 & 610 \\
\hline \multicolumn{17}{|c|}{ WITHIIV FIVE MILES } \\
\hline 1,3 & 48167 & & & & & 60 & & 200 & 330 & & & 260 & 390 & 75 & 430 & 610 \\
\hline 1,4 & 44436 & & & & & 60 & & 200 & 330 & & & 260 & 390 & & 440 & 620 \\
\hline 2,5 & $\mid 13229$ & & & & & 90 & & 200 & 330 & & & 290 & 420 & 120 & 430 & 610 \\
\hline 2,6 & 53410 & & & & & 90 & & 220 & 350 & & & 310 & 440 & 120 & 430 & 610 \\
\hline \multicolumn{17}{|c|}{ WITHIN TEN MILES } \\
\hline $1,3,7$ & 101378 & & & & & 90 & & 200 & 330 & & & 290 & 320 & 120 & 430 & 610 \\
\hline $1,4,8$ & 100002 & & & & & 60 & & 220 & 340 & & & 280 & 400 & & 430 & 610 \\
\hline $2,5,9$ & 32178 & & & & & 90 & & 200 & 330 & & & 290 & 320 & 120 & 430 & 610 \\
\hline $2,6,10$ & 12726 & & & & & 90 & & 220 & 360 & & & 310 & 450 & 120 & 430 & 610 \\
\hline
\end{tabular}

Note: Transients considered under special population category in this study. 
EVACUATION TIME ESTIMATES

Kewaunee

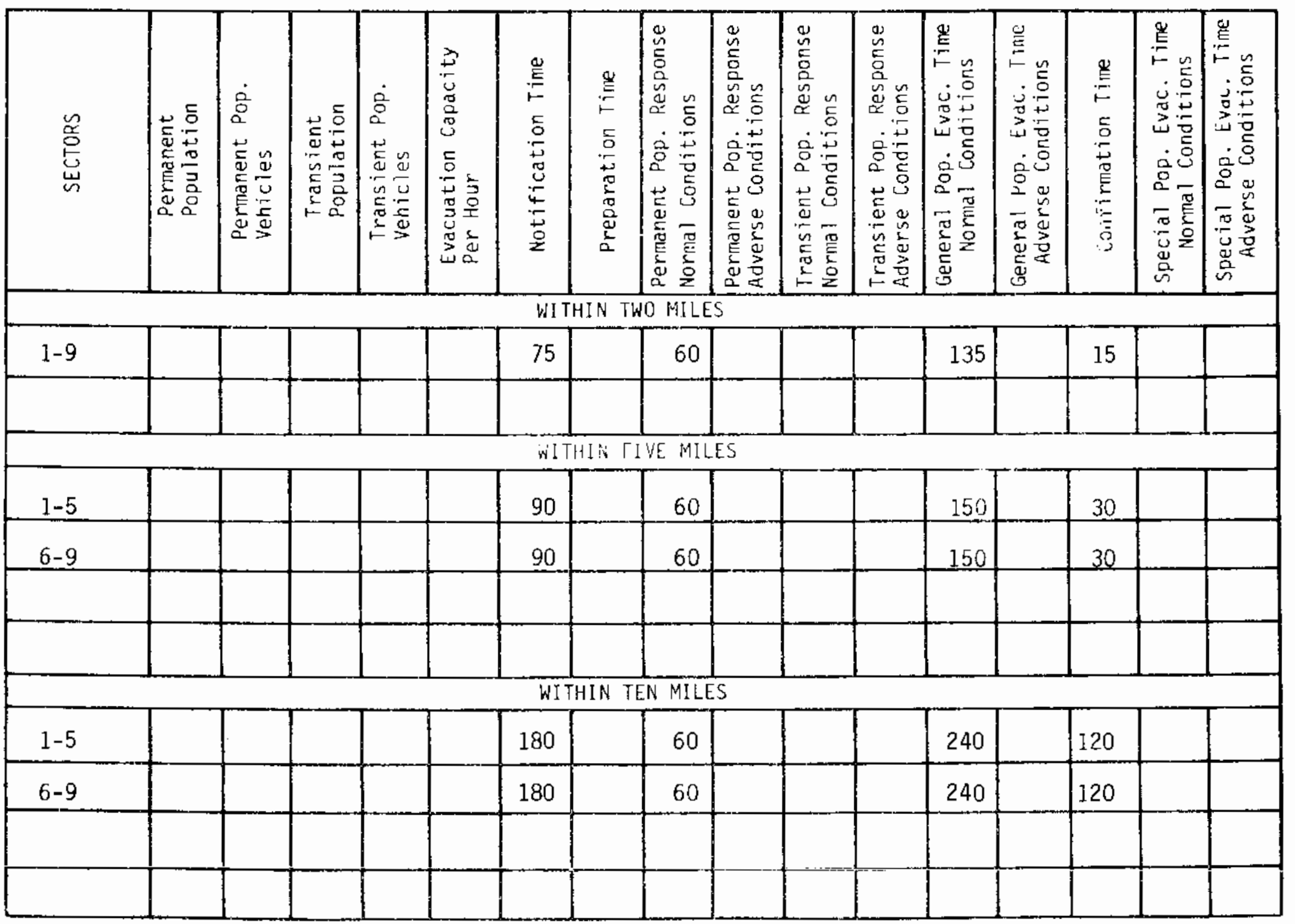


EVACUATION TIME ESTIMATES

Lacrosse

\begin{tabular}{|c|c|c|c|c|c|c|c|c|c|c|c|c|c|c|c|c|}
\hline $\begin{array}{l}\text { 愛 } \\
\text { 总 }\end{array}$ & 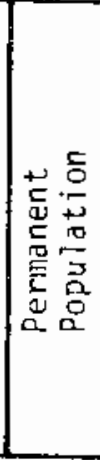 & 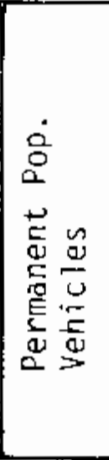 & 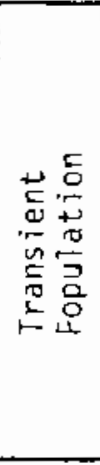 & 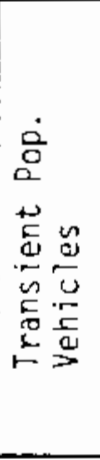 & 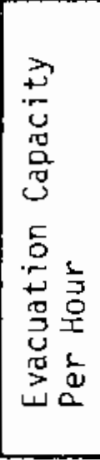 & 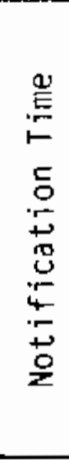 & 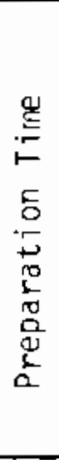 & 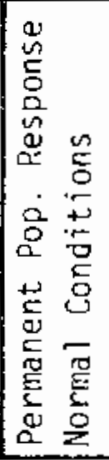 & 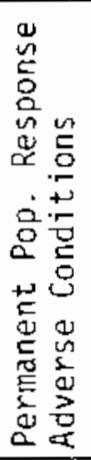 & 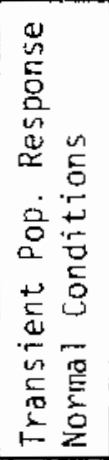 & 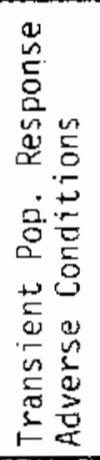 & 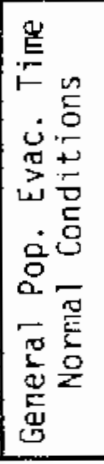 & 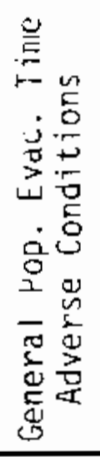 & 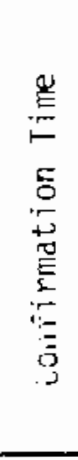 & 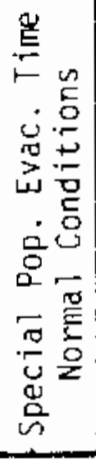 & 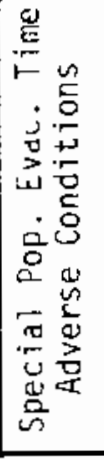 \\
\hline \multicolumn{17}{|c|}{ WITHIN TWO MILES } \\
\hline $\mathrm{E}$ & & & & & & 15 & & 15 & 45 & & & 30 & 60 & 60 & & \\
\hline$W$ & & & & & & - & & - & - & & & & & - & & \\
\hline \multicolumn{17}{|c|}{ WITtiIi FIVE MILES } \\
\hline $\mathrm{NE}$ & & & & & & 15 & & 15 & 45 & & & 30 & 60 & 60 & & \\
\hline SE & & & & & & 15 & & 30 & 75 & & & 45 & 90 & 60 & & \\
\hline$N W$ & & & & & & 15 & & 30 & 75 & & & 45 & 90 & 60 & & \\
\hline SW & & & & & & 15 & & 30 & 75 & & & 45 & 90 & 60 & & \\
\hline \multicolumn{17}{|c|}{ WITHIN TEN MILES } \\
\hline $\mathrm{NE}$ & & & & & & 15 & & 105 & 165 & & & 120 & 180 & 120 & & \\
\hline SE & & & & & & 15 & & 105 & 225 & & & 120 & 240 & 90 & & \\
\hline $\mathrm{NWW}$ & & & & & & 15 & & 105 & 225 & & & 120 & 240 & 120 & & \\
\hline SW & & & & & & 15 & & 105 & 225 & & & 120 & 240 & 120 & & \\
\hline
\end{tabular}


LaSalle

\begin{tabular}{|c|c|c|c|c|c|c|c|c|c|c|c|c|c|c|c|c|}
\hline 点 & 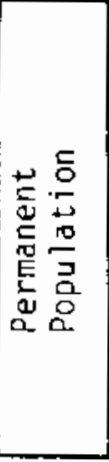 & 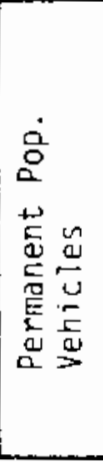 & 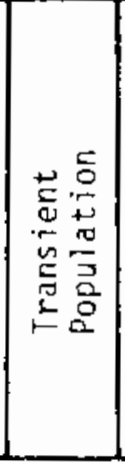 & 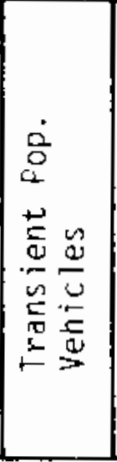 & 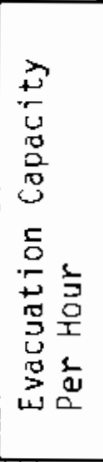 & 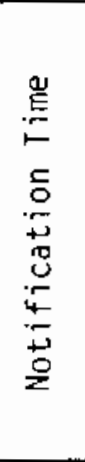 & 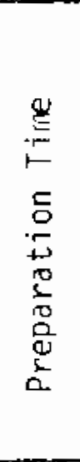 & 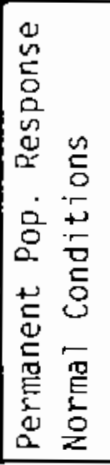 & 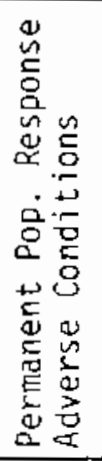 & 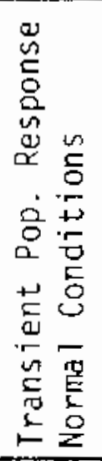 & 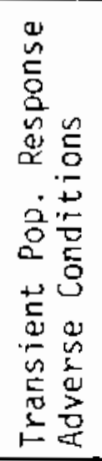 & 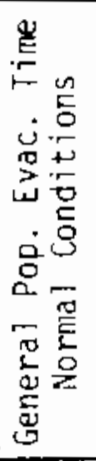 & 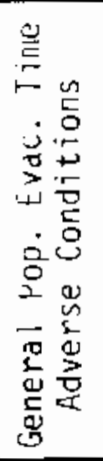 & 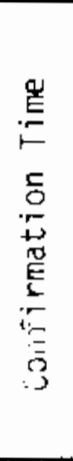 & 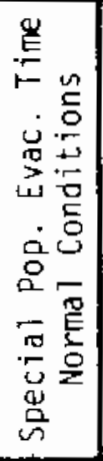 & 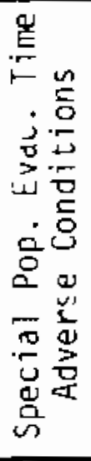 \\
\hline \multicolumn{17}{|c|}{ WITHIN TWO MILES } \\
\hline I & 30 & 10 & 520 & 520 & & & & & & & & 120 & & & & \\
\hline I I & 50 & 15 & & & & & & & & & & 60 & & & & \\
\hline \multicolumn{17}{|c|}{ WITHLA TIVE MILES } \\
\hline I, III & 505 & 170 & 520 & 520 & & 95 & 20 & 5 & & & & 120 & & & & \\
\hline$I, V I$ & 245 & 80 & 520 & 520 & & 90 & 20 & 10 & & & & 120 & & & & \\
\hline II, IV & 215 & 70 & & & & 65 & 20 & 35 & & & & 120 & & & & \\
\hline$I I, V$ & 195 & 65 & & & & 65 & 20 & 35 & & & & 120 & & & & \\
\hline \multicolumn{17}{|c|}{ WITHIN TEN MILES } \\
\hline$I, I I I, V I I$ & 4250 & 1415 & 1275 & 1275 & & 135 & 20 & 385 & & & & 540 & & & & \\
\hline$I, V I, X$ & 7635 & 2545 & 2070 & 2070 & & 165 & 20 & 295 & & & & 480 & & & 120 & \\
\hline II $, I V, V I I I$ & 1905 & 635 & 75 & 75 & & 85 & 20 & 495 & & & & 600 & & & & \\
\hline$I I, V, I X$ & 2870 & 935 & 230 & 230 & & 110 & 20 & 470 & & & & 600 & & & & \\
\hline
\end{tabular}

Note: Transients included in permanent population response time 
Maine yankee

\begin{tabular}{|c|c|c|c|c|c|c|c|c|c|c|c|c|c|c|c|c|}
\hline $\begin{array}{l}\text { 产 } \\
\text { 总 }\end{array}$ & 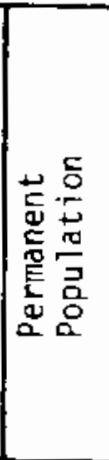 & 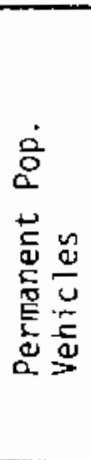 & 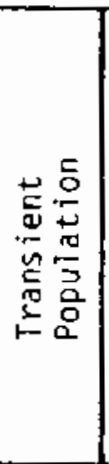 & 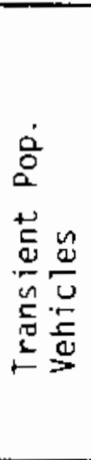 & 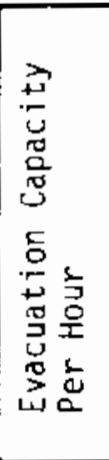 & 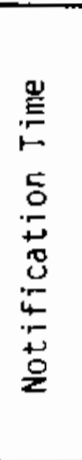 & 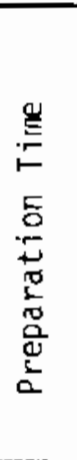 & 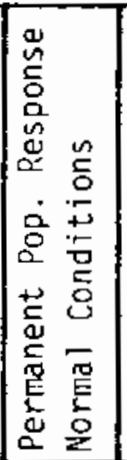 & 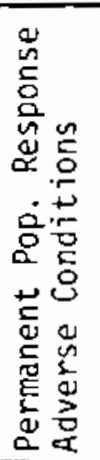 & 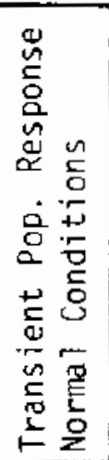 & 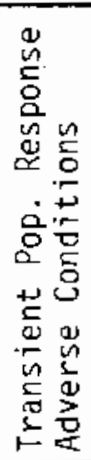 & 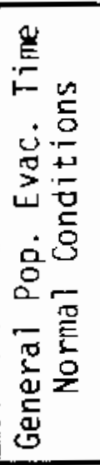 & 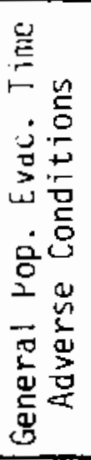 & 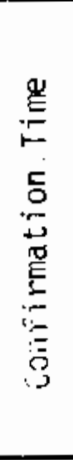 & 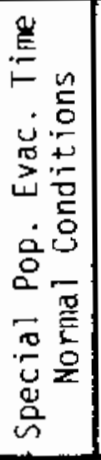 & 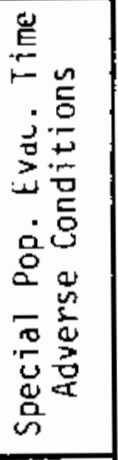 \\
\hline \multicolumn{17}{|c|}{ WITHIN TWO MILES } \\
\hline 1 & & & & & & 66 & & & & & & 78 & 90 & 60 & & \\
\hline 2 & & & & & & 66 & & & & & & 78 & 84 & 60 & & \\
\hline \multicolumn{17}{|c|}{ WITHIN FIVE MILES } \\
\hline 3 & & & & & & 66 & & & & & & 90 & 108 & 60 & & \\
\hline 4 & & & & & & 66 & & & & & & 108 & 132 & 60 & & \\
\hline 5 & & & & & & 66 & & & & & & 90 & 180 & 60 & & \\
\hline 6 & & & & & & 66 & & & & & & 102 & 120 & 60 & & \\
\hline \multicolumn{17}{|c|}{ WI THIN TEN MILES } \\
\hline 7 & & & & & & 66 & & & & & & 108 & 126 & 60 & & \\
\hline 8 & & & & & & 66 & & & & & & 120 & 144 & 60 & 72 & 78 \\
\hline 9 & & & & & & 66 & & & & & & 174 & 444 & 60 & 216 & 480 \\
\hline 10 & & & & & & 66 & & & & & & 228 & 270 & 60 & 270 & 312 \\
\hline
\end{tabular}




\begin{tabular}{|c|c|c|c|c|c|c|c|c|c|c|c|c|c|c|c|c|}
\hline $\begin{array}{l}\mathscr{2} \\
\stackrel{\mathscr{\delta}}{\varrho} \\
\text { 岕 }\end{array}$ & 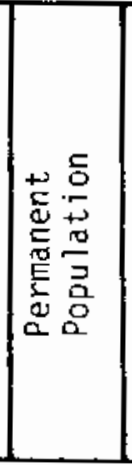 & 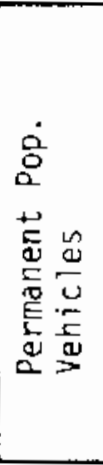 & 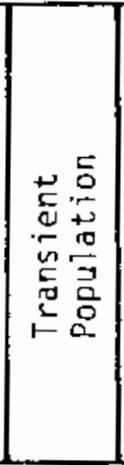 & 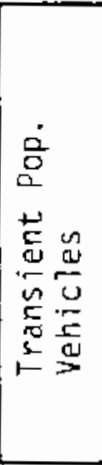 & 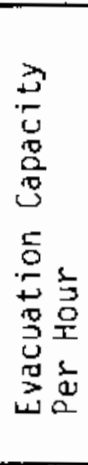 & 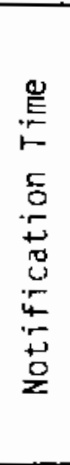 & 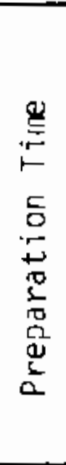 & 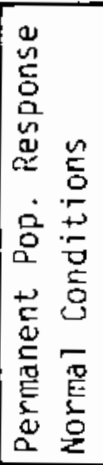 & 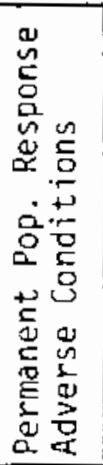 & 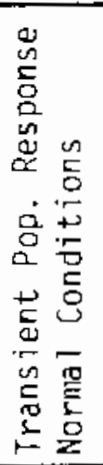 & 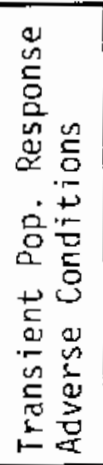 & 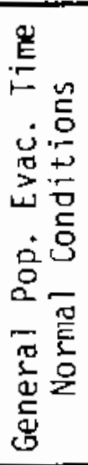 & 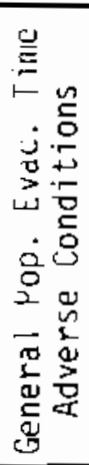 & 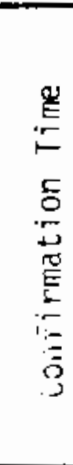 & 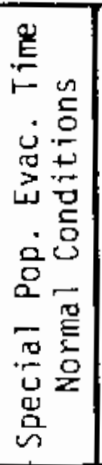 & 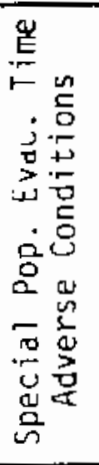 \\
\hline \multicolumn{17}{|c|}{ WITHIN TWO MILES } \\
\hline WSW-E & 318 & & 0 & & & 15 & & 20 & 35 & 0 & 0 & 35 & 50 & 50 & & \\
\hline ENE-W & 240 & & 3525 & & & 30 & & 45 & 60 & 0 & 0 & 75 & 90 & 45 & & \\
\hline \multicolumn{17}{|c|}{ WITHINA FIVE MILES } \\
\hline SSE-E & 1731 & & 0 & & & 15 & & 30 & 45 & & & 45 & 60 & 30 & & \\
\hline ENE-N & 1374 & & 12456 & & & 30 & & 55 & 70 & & & 85 & 100 & 45 & & \\
\hline NNW-W & 1225 & & 4336 & & & 30 & & 55 & 70 & & & 85 & 100 & 45 & & \\
\hline WSW-S & 1147 & & 0 & & & 15 & & 30 & 45 & & & 45 & 60 & 30 & & \\
\hline \multicolumn{17}{|c|}{ WITHIN TEN MILES } \\
\hline SSE-E & 19697 & & 3738 & & & 15 & & 45 & 65 & & & 60 & 80 & 60 & 105 & 135 \\
\hline ENE-N & 13368 & & 19618 & & & 15 & & 45 & 65 & & & 60 & 80 & 45 & & \\
\hline$N N W-W$ & 6496 & & 7159 & & & 15 & & 45 & 65 & & & 60 & 80 & 45 & & \\
\hline WSW-S & 25119 & & 663 & & & 15 & & 60 & 80 & & & 60 & 80 & 60 & & \\
\hline
\end{tabular}

Note: An apparent error exists as evacuation times are less for 10 mile radius than for 5 mile radius. 
Mi]lstone

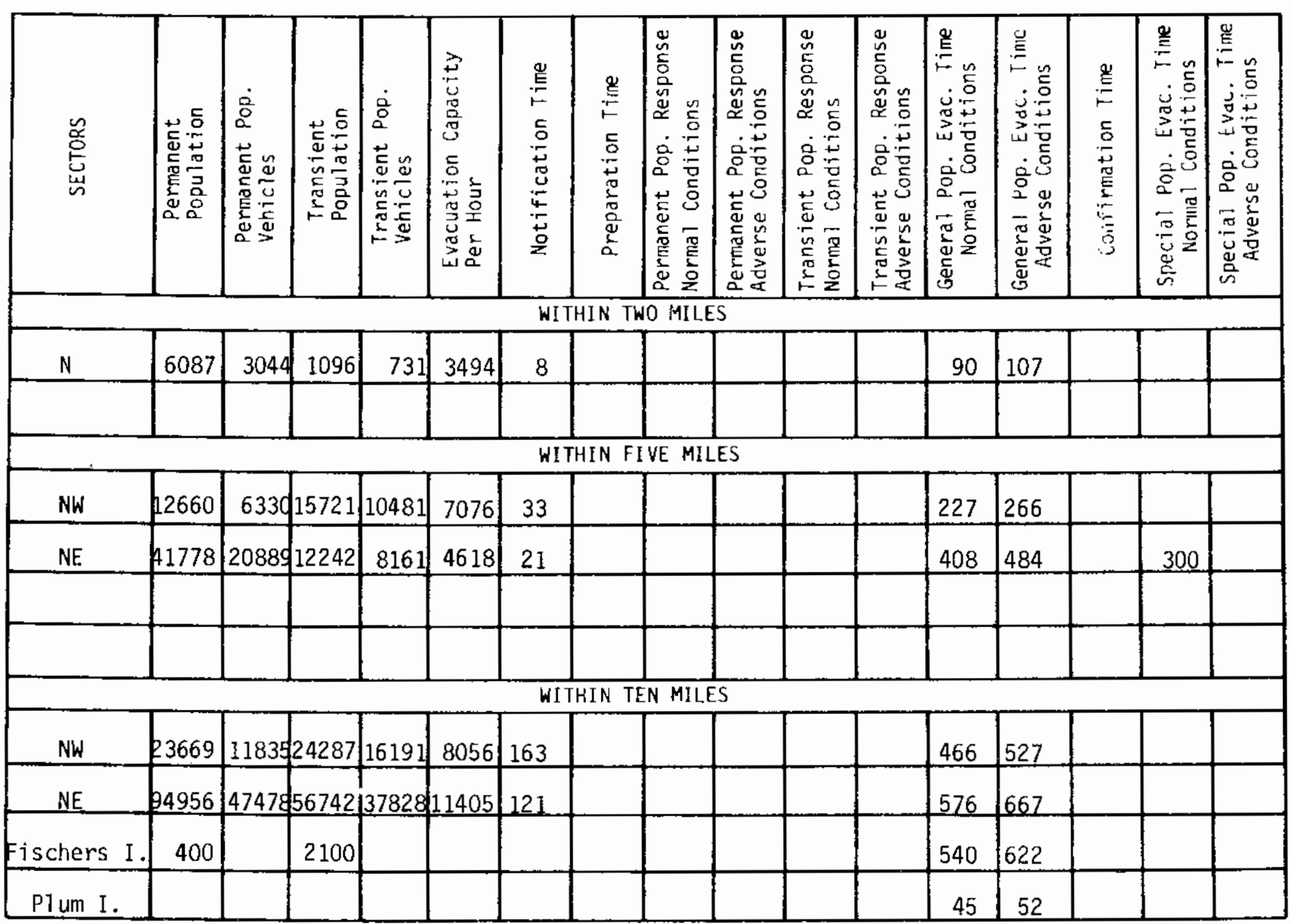


Monticello

\begin{tabular}{|c|c|c|c|c|c|c|c|c|c|c|c|c|c|c|c|c|}
\hline 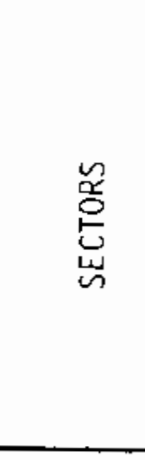 & 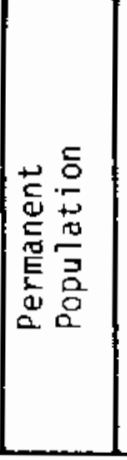 & 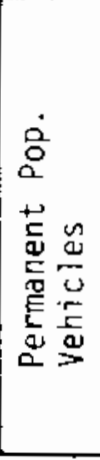 & 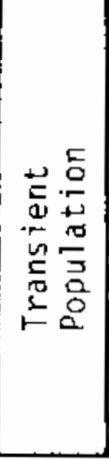 & 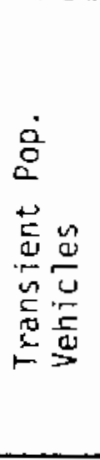 & 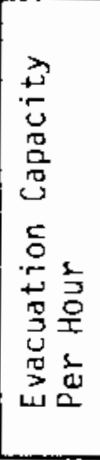 & 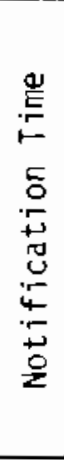 & 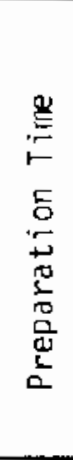 & 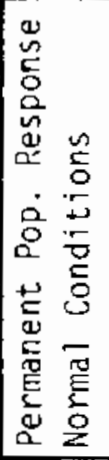 & 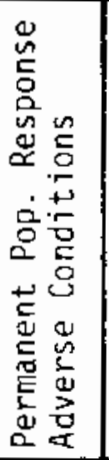 & 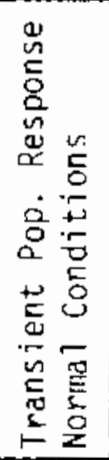 & 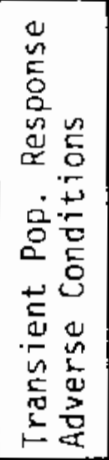 & 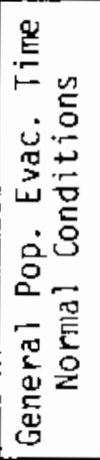 & 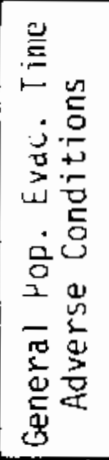 & 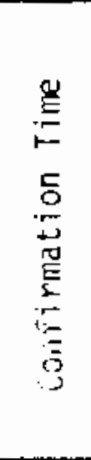 & 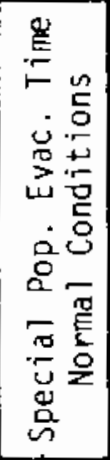 & 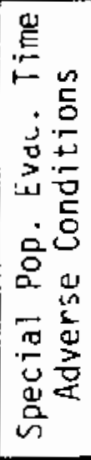 \\
\hline \multicolumn{17}{|c|}{ WITHIN TWO MILES } \\
\hline NW-ESE & 266 & & & & & & & 10 & 15 & & & & & 30 & & \\
\hline SE-WNW & 509 & & & & & & & 15 & 20 & & & & & 40 & & \\
\hline \multicolumn{17}{|c|}{ GIThIS FIVE MILES } \\
\hline $\mathrm{NW}-\mathrm{NNE}$ & 1099 & & & & & & & 30 & 40 & & & & & 80 & & \\
\hline NE-ESE & 1766 & & & & & & & 35 & 45 & & & & & 90 & & \\
\hline SE-SSW & 4573 & & & & & & & 45 & 54 & & & & & 110 & & \\
\hline SW-WNW & 1133 & & & & & & & 30 & 40 & & & & & 80 & & \\
\hline \multicolumn{17}{|c|}{ WITHIN TEN MILES } \\
\hline NW-NNE & 2855 & & & & & & & 60 & 80 & & & & & 160 & & \\
\hline NE-ESE & 6609 & & & & & & & 75 & 100 & & & & & 200 & & \\
\hline SE-SSW & 8890 & & & & & & & 90 & 120 & & & & & 240 & 200 & \\
\hline SW-WNW & 3620 & & & & & & & 65 & 85 & & & & & 170 & & \\
\hline
\end{tabular}




\begin{tabular}{|c|c|c|c|c|c|c|c|c|c|c|c|c|c|c|c|c|}
\hline 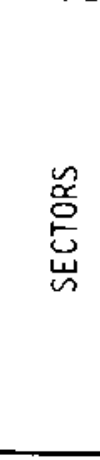 & 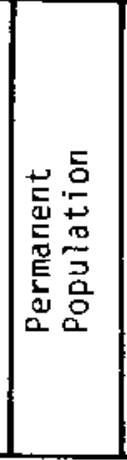 & 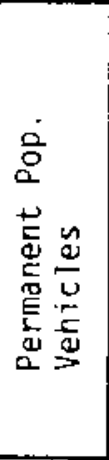 & 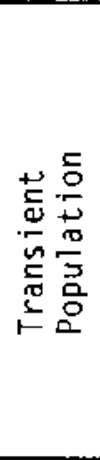 & 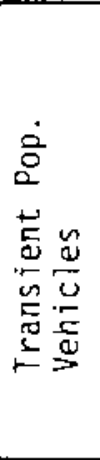 & 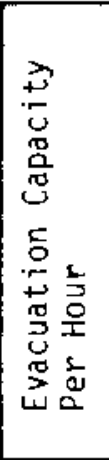 & 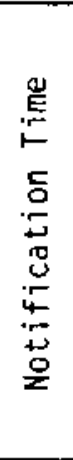 & 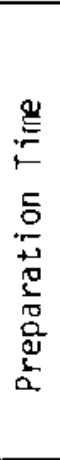 & 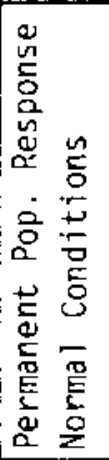 & 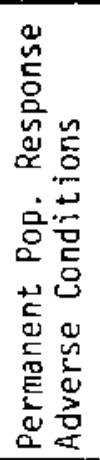 & 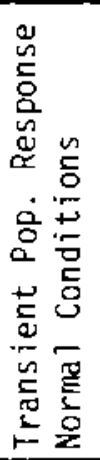 & 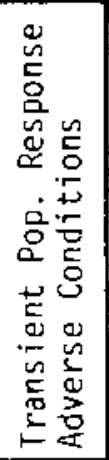 & 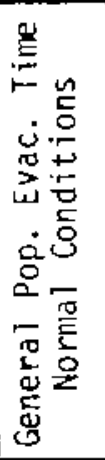 & 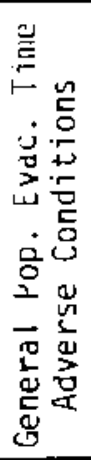 & 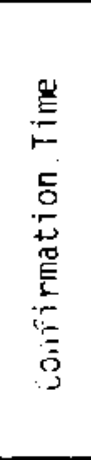 & 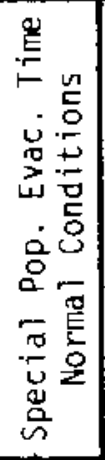 & 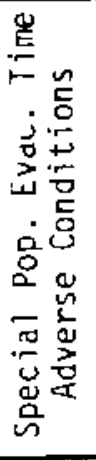 \\
\hline \multicolumn{17}{|c|}{ WI THIN TWO MILES } \\
\hline $\mathrm{s}$ & 1088 & & & & & 60 & & 70 & 90 & & & 130 & 150 & 90 & & \\
\hline \multicolumn{17}{|c|}{ WITHIN FIVE MILES } \\
\hline SW & 3733 & & & & & 60 & & 70 & 100 & & & 130 & 160 & 150 & & \\
\hline SE & 2824 & & & & & 60 & & 70 & 100 & & & 130 & 160 & 150 & & \\
\hline & & & & & & & & & & & & & & & & \\
\hline & & & & & & & & & & & & & & & & \\
\hline \multicolumn{17}{|c|}{ WITHIN TEN MILES } \\
\hline SW & 35973 & & & & & 60 & & 170 & 280 & & & 230 & 340 & 210 & 130 & 210 \\
\hline SE & 9575 & & & & & 60 & & 90 & 120 & & & 150 & 180 & 210 & & \\
\hline lake & & & & & & & & & & & & & & & 45 & \\
\hline A11 & 43772 & & & & & 60 & & 170 & 280 & & & 230 & 340 & 360 & 130 & 210 \\
\hline
\end{tabular}

Note: Same location as Fitzpatrick 
North Anna

\begin{tabular}{|c|c|c|c|c|c|c|c|c|c|c|c|c|c|c|c|c|}
\hline 号 & 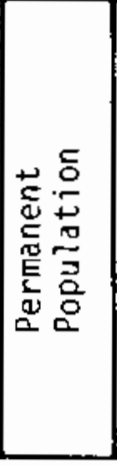 & 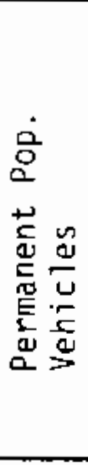 & 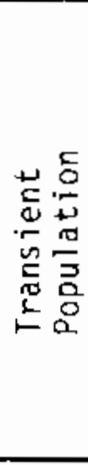 & 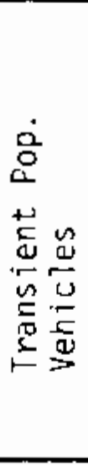 & 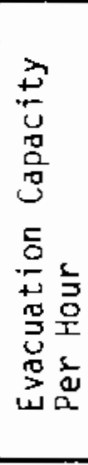 & 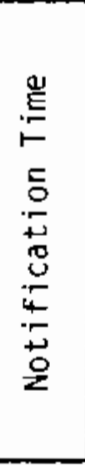 & 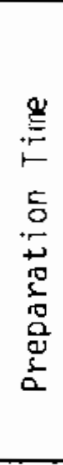 & 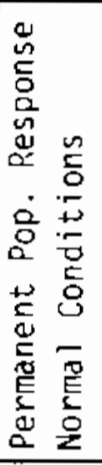 & 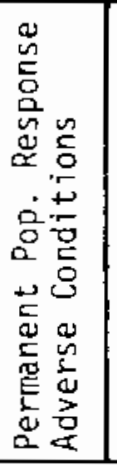 & 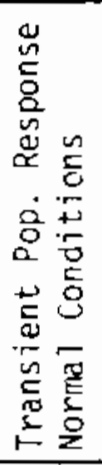 & 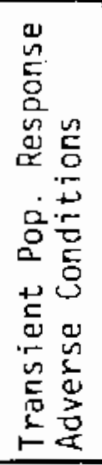 & 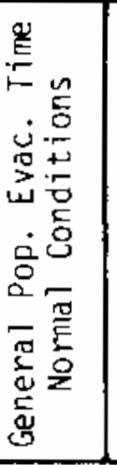 & 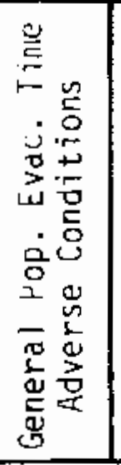 & 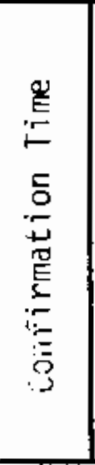 & 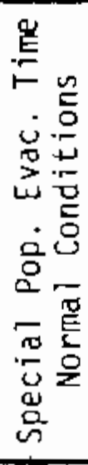 & 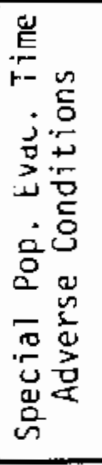 \\
\hline \multicolumn{17}{|c|}{ WITHIN TWO MILES } \\
\hline$A-H$ & 72 & & & & & 45 & & 45 & 60 & & & 90 & 105 & 60 & 0 & 0 \\
\hline$J-R$ & 50 & & & & & 120 & & 180 & 300 & & & 300 & $42 d$ & 360 & 0 & 0 \\
\hline \multicolumn{17}{|c|}{ WITHIN FIVE MILES } \\
\hline$A-D$ & 839 & & & & & 90 & & 90 & 135 & & & 180 & 225 & 135 & 30 & 45 \\
\hline$E-H$ & 278 & & & & & 60 & & 90 & 150 & & & 150 & 210 & 240 & 0 & 0 \\
\hline$J-M$ & 1860 & & & & & 120 & & 180 & 420 & & & 300 & 540 & 720 & 0 & 0 \\
\hline$N-R$ & 1976 & & & & & 120 & & 240 & 420 & & & 360 & 540 & 720 & 0 & 0 \\
\hline \multicolumn{17}{|c|}{ WITHIN TEN MILES } \\
\hline$A=D$ & 2966 & & & & & 180 & & 180 & 260 & & & 360 & 440 & 260 & 24 & 36 \\
\hline $\mathrm{E}-\mathrm{H}$ & 3104 & & & & & 180 & & 180 & 270 & & & 360 & 450 & 480 & 30 & 45 \\
\hline$J-M$ & 2800 & & & & & 240 & & 180 & 720 & & & 420 & 960 & 1440 & 0 & 0 \\
\hline$N-R$ & 3754 & & & & & 240 & & 240 & 720 & & & 480 & 960 & 1440 & 0 & 0 \\
\hline
\end{tabular}




\begin{tabular}{|c|c|c|c|c|c|c|c|c|c|c|c|c|c|c|c|c|}
\hline $\begin{array}{l}\text { 㟧 } \\
\text { 总 } \\
\text { 出 }\end{array}$ & 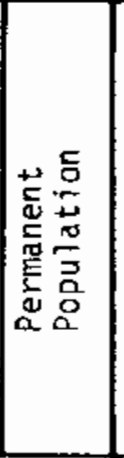 & 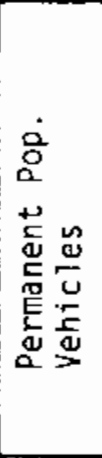 & 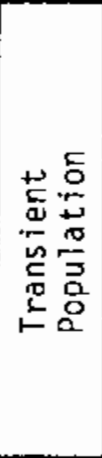 & 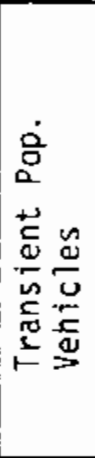 & 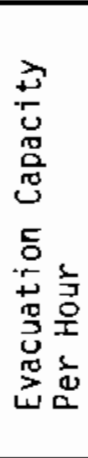 & 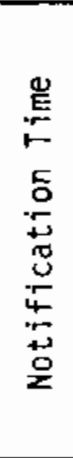 & 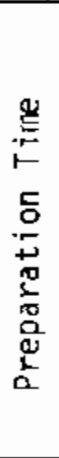 & 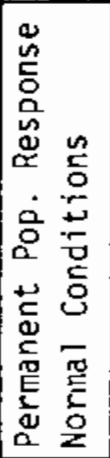 & 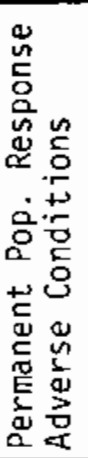 & 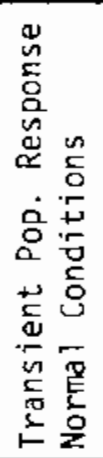 & 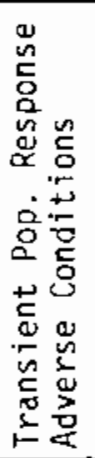 & 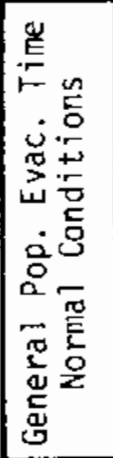 & 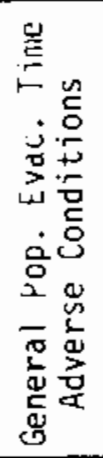 & 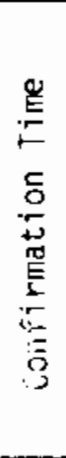 & 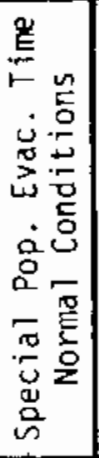 & 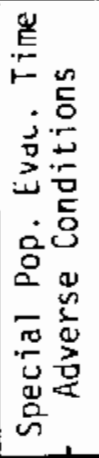 \\
\hline \multicolumn{17}{|c|}{ WITHIN TWO MILES } \\
\hline NNE-S & 225 & & 445 & & & 15 & & & & & & 60 & 80 & 20 & & \\
\hline SSW-N & 63 & & 2711 & & & 20 & & & & & & 60 & 70 & 20 & & \\
\hline \multicolumn{17}{|c|}{ WITHIN FIVE MILES } \\
\hline NNE-E & 1961 & & 264 & & & 45 & & & & & & 180 & 240 & 30 & 210 & 300 \\
\hline ESE-S & 1444 & & 546 & & & 35 & & & & & & 90 & 120 & 30 & & \\
\hline SSW-W & 1086 & & 1775 & & & 45 & & & & & & 90 & 120 & 30 & & \\
\hline$W N W-N$ & 500 & & 3923 & & & 30 & & & & & & 90 & 120 & 20 & & \\
\hline \multicolumn{17}{|c|}{ WITHIN TEN MILES } \\
\hline NNE - E & 7822 & & 588 & & & 75 & & & & & & 210 & 270 & 50 & & \\
\hline ESE-S & 23888 & & 3283 & & & 60 & & & & & & 180 & 250 & 50 & & \\
\hline$S S W-W$ & 17495 & & 3670 & & & 75 & & & & & & 210 & 270 & 50 & 500 & 590 \\
\hline WNW-N & 4279 & & 5483 & & & 75 & & & & & & 210 & 270 & 50 & & \\
\hline
\end{tabular}

Note: Data may not be cumulative for evacuation times. 
EVACIIATION TIMF FSTIMATES

Oys ter Creek

\begin{tabular}{|c|c|c|c|c|c|c|c|c|c|c|c|c|c|c|c|c|}
\hline 琶 & 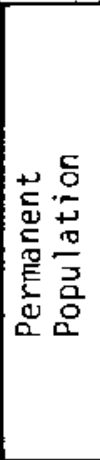 & 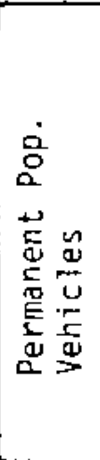 & 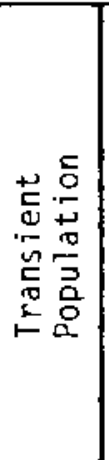 & 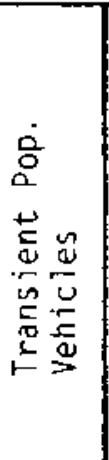 & 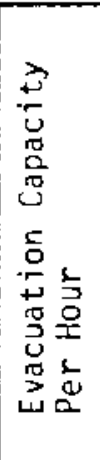 & 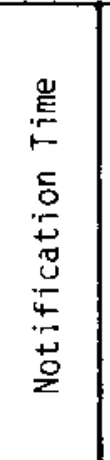 & 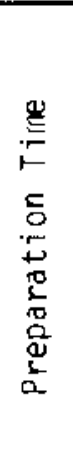 & 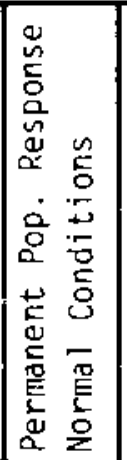 & 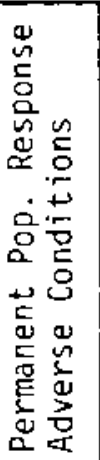 & 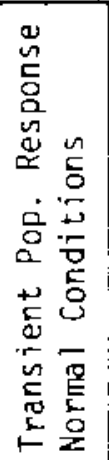 & 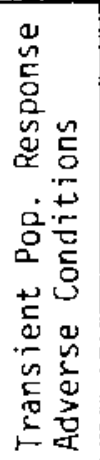 & 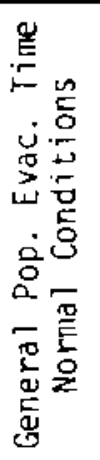 & 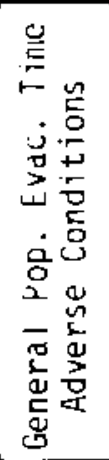 & 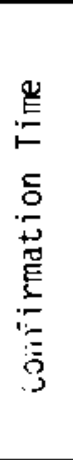 & 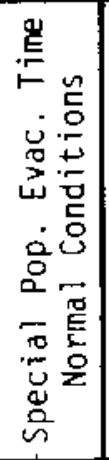 & 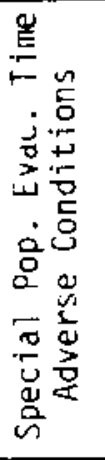 \\
\hline \multicolumn{17}{|c|}{ WI THIN TWO MILES } \\
\hline A & & & & & & 60 & & 30 & 75 & & & 90 & 135 & & 90 & 120 \\
\hline B & & & & & & 60 & & 102 & 183 & & & 162 & 243 & & & \\
\hline \multicolumn{17}{|c|}{ NITHIV FIVE MILES } \\
\hline $\mathrm{NE}$ & & & & & & 60 & & 150 & 255 & & & 210 & 315 & & 90 & 120 \\
\hline$S E$ & & & & & & 60 & & 60 & 120 & & & 120 & 180 & & & \\
\hline SW & & & & & & 60 & & 30 & 75 & & & 90 & 135 & & 120 & 120 \\
\hline $\mathrm{NW}$ & & & & & & 60 & & 48 & 102 & & & 108 & 162 & & & \\
\hline \multicolumn{17}{|c|}{ WITHIN TEN MILES } \\
\hline $\mathrm{NE}$ & & & & & & 120 & & 240 & 420 & & & 360 & 540 & & 90 & 120 \\
\hline $\mathrm{SE}$ & & & & & & 120 & & 84 & 186 & & & 204 & 306 & & & \\
\hline SW & & & & & & 120 & & 30 & 105 & & & 150 & 225 & & 90 & 120 \\
\hline $\mathrm{NW}$ & & & & & & 120 & & 96 & 204 & & & 216 & 324 & & 90 & 120 \\
\hline
\end{tabular}


EVACUATION TIME ESTIMATES

Palisades

\begin{tabular}{|c|c|c|c|c|c|c|c|c|c|c|c|c|c|c|c|c|}
\hline 哭 & 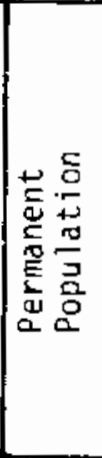 & 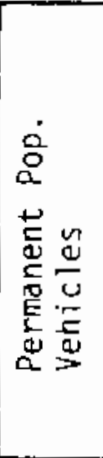 & 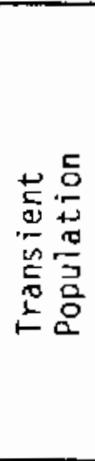 & 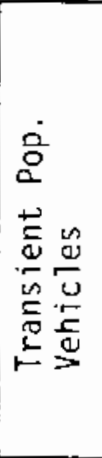 & 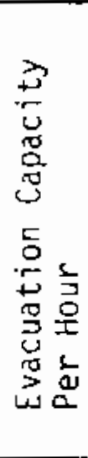 & 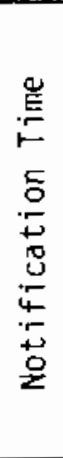 & 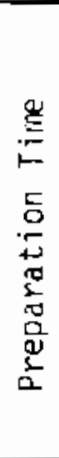 & 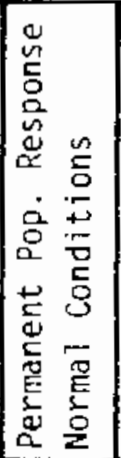 & 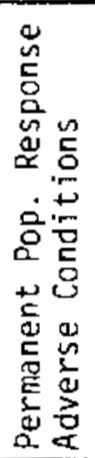 & 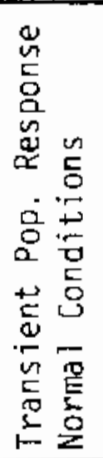 & 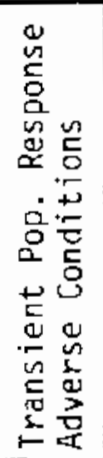 & 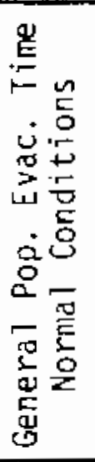 & 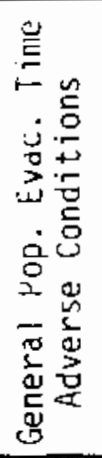 & 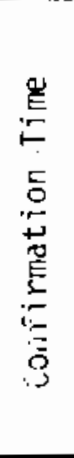 & 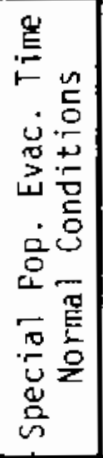 & 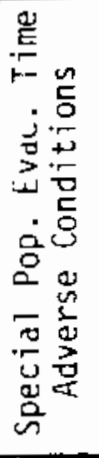 \\
\hline \multicolumn{17}{|c|}{ WITHIN TWO MILES } \\
\hline$N-S S W$ & & & & & & & & 31 & 11 & & & & & & & \\
\hline & & & & & & & & & & & & & & & & \\
\hline \multicolumn{17}{|c|}{ WITHIN FIVE MILES } \\
\hline ESE-SSW & & & & & & & & 28 & 29 & & & & & & & \\
\hline$N-E$ & & & & & & & & 58 & 135 & & & & & & & \\
\hline & & & & & & & & & & & & & & & & \\
\hline & & & & & & & & & & & & & & & & \\
\hline \multicolumn{17}{|c|}{ WITHIN YEN MILES } \\
\hline ESE-SSW & & & & & & & & 120 & 124 & & & & & & & \\
\hline$N-E$ & & & & & & & & 158 & 165 & & & & & & & \\
\hline & & & & & & & & & & & & & & & & \\
\hline & & & & & & & & & & & & & & & & \\
\hline
\end{tabular}


EVACLAATION TIHE ESTIMATES

Peach Bottom

\begin{tabular}{|c|c|c|c|c|c|c|c|c|c|c|c|c|c|c|c|c|}
\hline 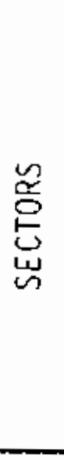 & 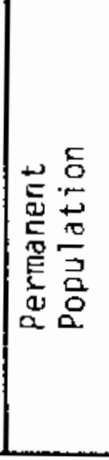 & 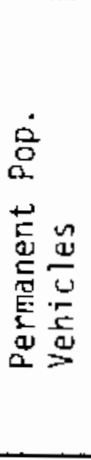 & 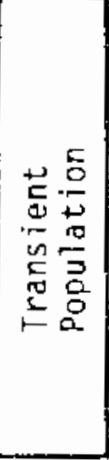 & 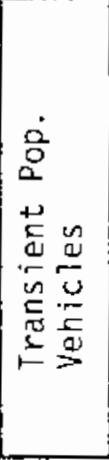 & 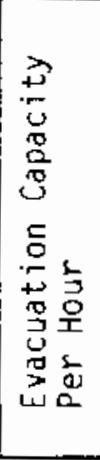 & 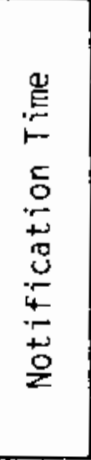 & 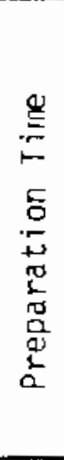 & 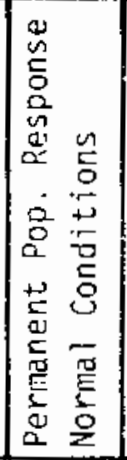 & 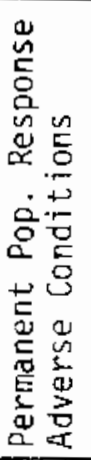 & 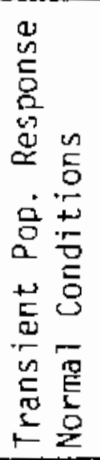 & 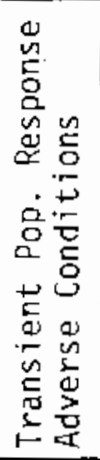 & 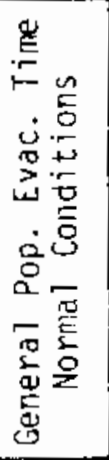 & 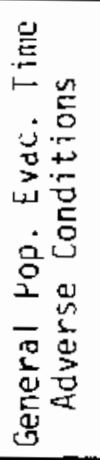 & 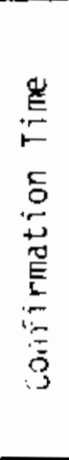 & 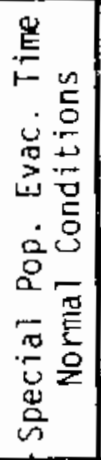 & 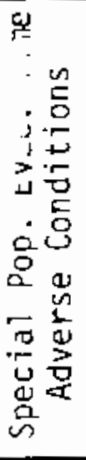 \\
\hline \multicolumn{17}{|c|}{ WITHIN TWO MILES } \\
\hline NE & & & & & & & & 60 & 120 & & & & & & & \\
\hline SW & & & & & & & & 60 & 120 & & & & & & & \\
\hline \multicolumn{17}{|c|}{ WITHI IV FIVE MILES } \\
\hline $\mathrm{N}$ & & & & & & & & 120 & 240 & & & & & & & \\
\hline E & & & & & & & & 120 & 240 & & & & & & & \\
\hline $\mathrm{s}$ & & & & & & & & 120 & 240 & & & & & & & \\
\hline$\omega$ & & & & & & & & 120 & 240 & & & & & & & \\
\hline \multicolumn{17}{|c|}{ WITHIN TEN MILES } \\
\hline $\mathrm{N}$ & & & & & & 180 & & 180 & 540 & & & 360 & 720 & & & \\
\hline E & & & & & & 180 & & 180 & 540 & & & 360 & 720 & & & \\
\hline $\mathrm{s}$ & & & & & & 180 & & 120 & 360 & & & 300 & 540 & & & \\
\hline$w$ & & & & & & 180 & & 120 & 360 & & & 300 & 540 & & & \\
\hline
\end{tabular}


Pilarim Station

\begin{tabular}{|c|c|c|c|c|c|c|c|c|c|c|c|c|c|c|c|c|}
\hline 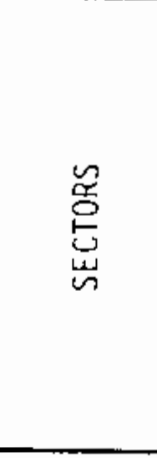 & 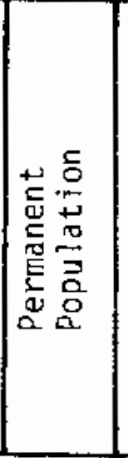 & 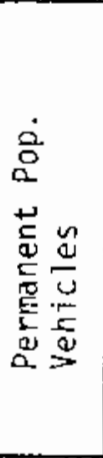 & 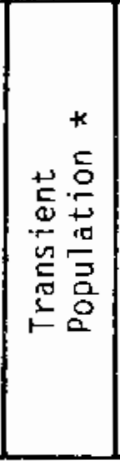 & 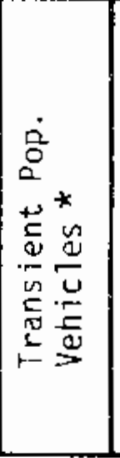 & 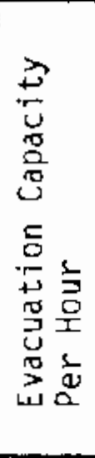 & 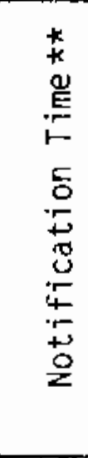 & 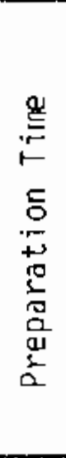 & 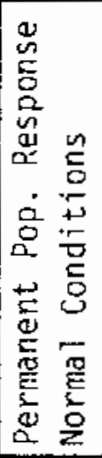 & 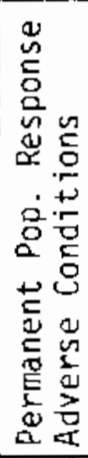 & 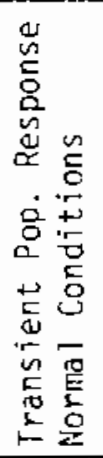 & 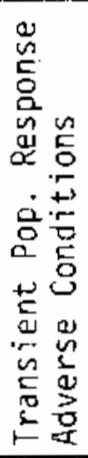 & 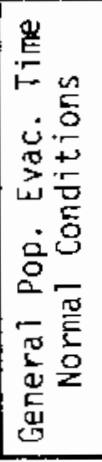 & 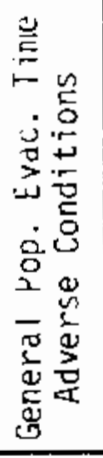 & 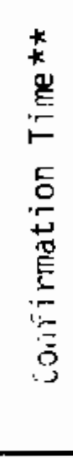 & 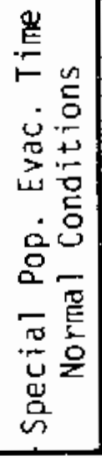 & 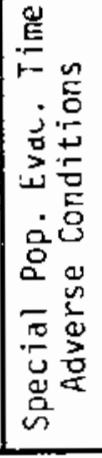 \\
\hline \multicolumn{17}{|c|}{ WITHIN TWO MILES } \\
\hline SE-NW & 2235 & 894 & $\begin{array}{l}4690 \\
2959 \\
\end{array}$ & \begin{tabular}{|r|}
1173 \\
987 \\
\end{tabular} & & $\frac{35}{45}$ & & 44 & 75 & 106 & & 185 & 120. & $\frac{35}{45}$ & & \\
\hline \multicolumn{17}{|c|}{ WITHIN FIVE MILES } \\
\hline NNW-NW & 2241 & 897 & $\begin{array}{l}5748 \\
2959 \\
\end{array}$ & $\begin{array}{r}1437 \\
987 \\
\end{array}$ & & $\frac{35}{45}$ & & 32 & 120 & 88 & & 355 & 165 & $\frac{35}{45}$ & & \\
\hline WNW-SW & 10938 & 4160 & $\begin{array}{r}5165 \\
15014\end{array}$ & $\begin{array}{l}1292 \\
5005\end{array}$ & & $\frac{45}{60}$ & & 86 & 170 & 129 & & 360 & 230 & $\frac{45}{60}$ & & \\
\hline SSW-SE & 5619 & 2248 & \begin{tabular}{|l|}
9525 \\
5210 \\
\end{tabular} & \begin{tabular}{|l|}
2382 \\
1737 \\
\end{tabular} & & $\frac{35}{45}$ & & 100 & 100 & 185 & & 320 & 145 & $\frac{35}{45}$ & & \\
\hline \multicolumn{17}{|c|}{ WITHIN TEN MILES } \\
\hline $\mathrm{NNW}-\mathrm{NW}$ & 1414 & 8566 & $\begin{array}{r}9219 \\
13054 \\
\end{array}$ & $\begin{array}{l}2305 \\
5018\end{array}$ & & $\frac{35}{45}$ & & 218 & 265 & 187 & & 440 & 310 & $\frac{35}{45}$ & & \\
\hline WNW-SW & 35394 & 14158 & $\begin{array}{r}9862 \\
25009\end{array}$ & $\begin{array}{l}2466 \\
8337\end{array}$ & & $\frac{45}{60}$ & & 119 & 175 & 91 & & 255 & 235 & $\frac{45}{60}$ & & \\
\hline SSW-SE & 6625 & 2650 & $\begin{array}{l}14302 \\
11639\end{array}$ & $\begin{array}{l}3576 \\
3880 \\
\end{array}$ & & $\frac{35}{45}$ & & 79 & 125 & $22 I$ & & 335 & 170 & $\frac{35}{45}$ & & \\
\hline & & & & & & & & & & & & & & & & \\
\hline
\end{tabular}

Notes: * Transient includes seasonal (Top) and transient (Bottom) ** Norma1 (Top)/Adverse (Bottom) 
Point Beach

\begin{tabular}{|c|c|c|c|c|c|c|c|c|c|c|c|c|c|c|c|c|}
\hline 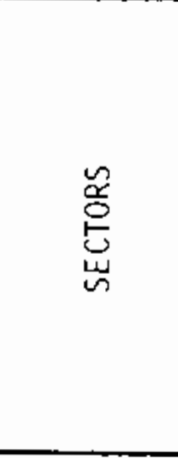 & 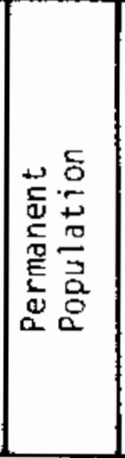 & 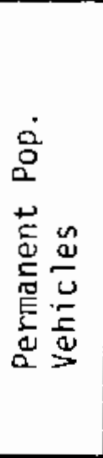 & 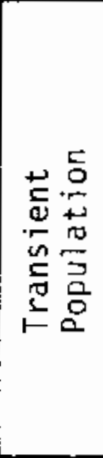 & 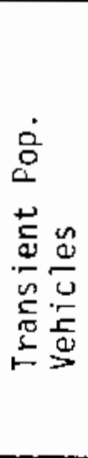 & 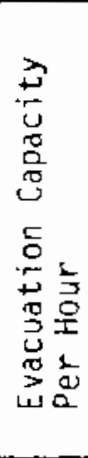 & 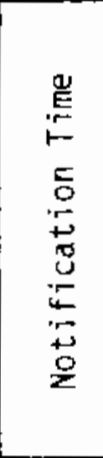 & 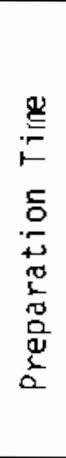 & 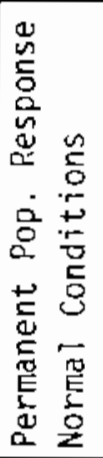 & 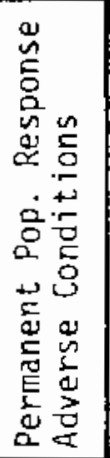 & 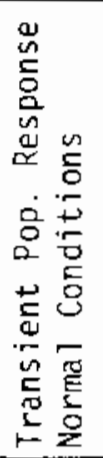 & 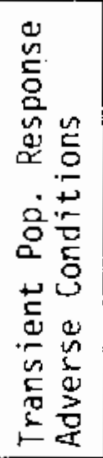 & 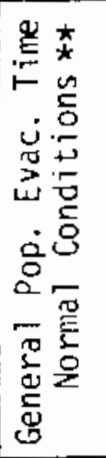 & 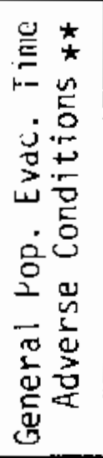 & 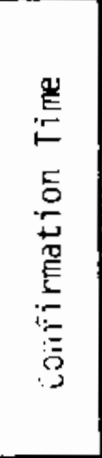 & 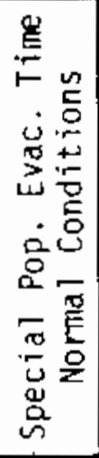 & 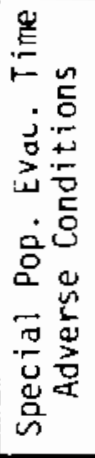 \\
\hline \multicolumn{17}{|c|}{ WITHIN TWO MILES } \\
\hline $\mathrm{LPZ}$ & 265 & 90 & & & & $\frac{25}{60 *}$ & & 52 & 110 & & & 55 & 120 & $\frac{15}{30} \pi$ & & \\
\hline \multicolumn{17}{|c|}{ WITHIiV FIVE MILES $\star \star \star \star$} \\
\hline$N W-N$ & 338 & 97 & & & & $\frac{25}{50} *$ & & 50 & 110 & & & 55 & 120 & $\frac{15}{30^{\star}}$ & & \\
\hline SW-WNW & 519 & 150 & & & & $\frac{40}{90 *}$ & & 65 & 340 & & & 75 & 150 & $\frac{25}{50} \star$ & & \\
\hline SSE-SSW & 388 & 110 & & & & $\frac{25}{60^{\star}}$ & & 30 & 110 & & & 55 & 120 & $\frac{15}{30}{ }^{*}$ & & \\
\hline \multicolumn{17}{|c|}{ 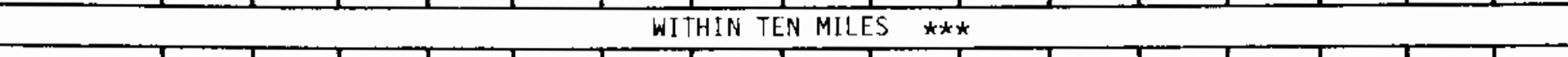 } \\
\hline$N W-N$ & 1613 & 447 & & & & $\frac{60}{130} \star$ & & 65 & 180 & & & 100 & 200 & $\frac{40}{60 *}$ & & \\
\hline SW-WWWL & 4339 & 1500 & & & & $\frac{140}{280 *}$ & & 165 & 330 & & & 210 & & $\frac{70}{120} \star$ & & \\
\hline SSE-SSW & 15738 & 4610 & 1200 & 250 & & $\frac{120}{200^{*}}$ & & 210 & 450 & 135 & 50 & 240 & 480 & $\frac{120}{120 *}$ & & \\
\hline & & & & & & & & & & & & & & & & \\
\hline
\end{tabular}

Notes: $\quad$ Normal (Top)/Adverse (Bottom)

** Total General Population Evacuation Time estimated to be non-cumulative

$\star \star \star$ Estimates for 5 and $10-$ mile radius mabe non-cumulative 
Prairie Island

\begin{tabular}{|c|c|c|c|c|c|c|c|c|c|c|c|c|c|c|c|c|}
\hline 号 & 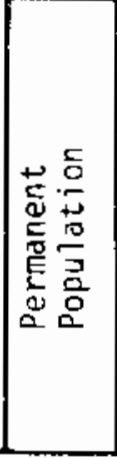 & 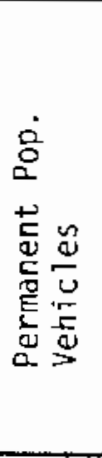 & 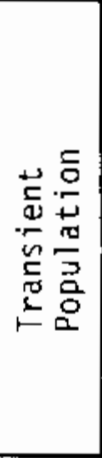 & 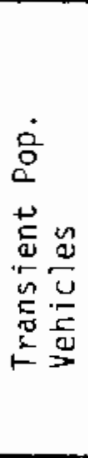 & 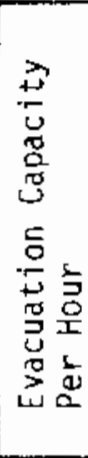 & 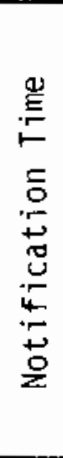 & 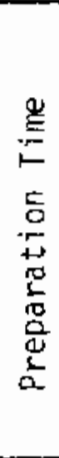 & 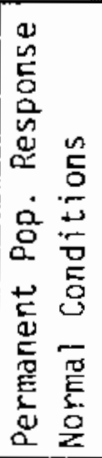 & 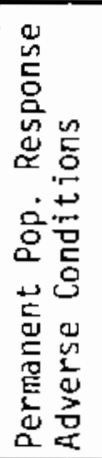 & 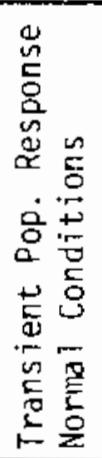 & 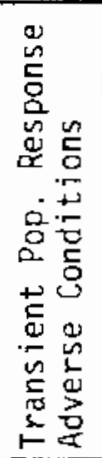 & 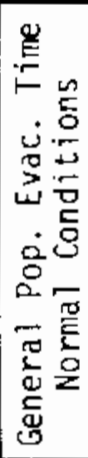 & 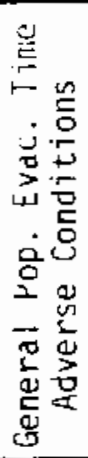 & 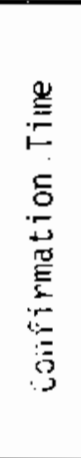 & 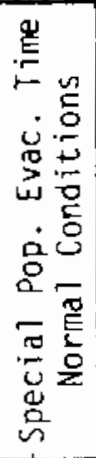 & 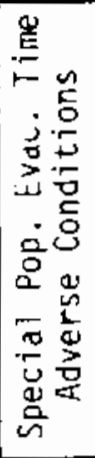 \\
\hline \multicolumn{17}{|c|}{ WITHIN TWO MILES } \\
\hline $\mathrm{NW}-\mathrm{ESE}$ & 238 & 119 & & & & & & 15 & 20 & & & 30 & 35 & 40 & & \\
\hline SE-WNW & 111 & 56 & & & & & & 15 & 20 & & & 30 & 35 & 40 & & \\
\hline \multicolumn{17}{|c|}{ WITHIN FIVE MILES } \\
\hline NW-NNE & 370 & 185 & & & & & & 30 & 40 & & & 45 & 55 & 80 & & \\
\hline NE-ESE & 610 & 305 & & & & & & 30 & 40 & & & 45 & 55 & مهـ & & \\
\hline SE-SSW & 2302 & 1151 & & & & & & 40 & 52 & & & 55 & 67 & 100 & & \\
\hline SW-WNW & 378 & 189 & & & & & & 30 & 40 & & & 45 & 55 & 80 & & \\
\hline \multicolumn{17}{|c|}{ WITHIN TEN MILES } \\
\hline NW-NNE & 2153 & 1077 & & & & & & 60 & 80 & & & 75 & 95 & 160 & & \\
\hline NE-ESE & 4355 & 2178 & & & & & & 50 & 65 & & & 65 & 80 & 130 & & \\
\hline SE-SSW & 14016 & 7008 & & & & & & 90 & 120 & & & 105 & 135 & 240 & & \\
\hline SW-WNW & 1790 & 895 & & & & & & 60 & 80 & & & 75 & 95 & 160 & & \\
\hline
\end{tabular}

Note: Notification time assumed to be 15 minutes to compute total general population evacuation time 


\section{EVACUATION TIME ESTIMATES}

Quad Cities

\begin{tabular}{|c|c|c|c|c|c|c|c|c|c|c|c|c|c|c|c|c|}
\hline $\begin{array}{l}\mathscr{2} \\
\stackrel{0}{\circ} \\
\text { 岀 }\end{array}$ & 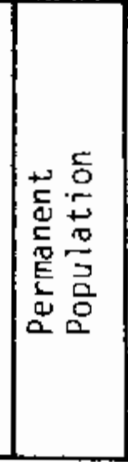 & 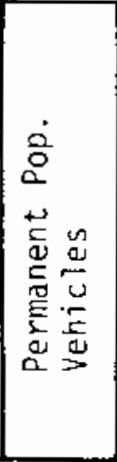 & 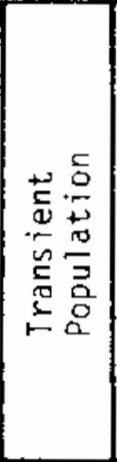 & 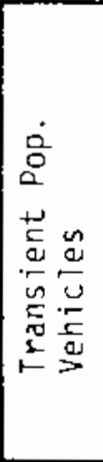 & 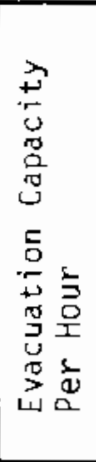 & 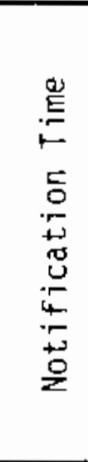 & 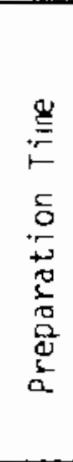 & 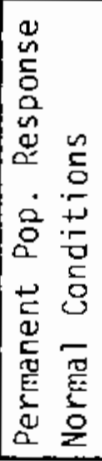 & 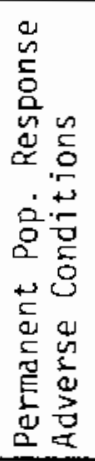 & 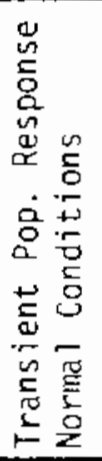 & 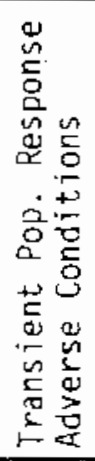 & 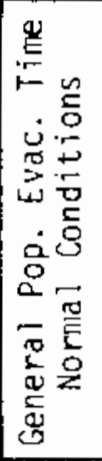 & 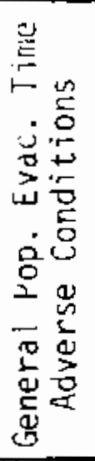 & 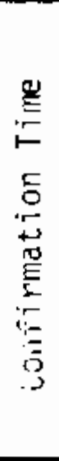 & 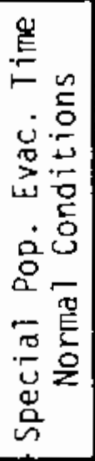 & 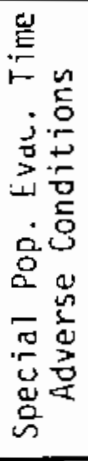 \\
\hline \multicolumn{17}{|c|}{ WITHIN TWO MILES } \\
\hline I & 45 & 15 & & 0 & & 35 & 20 & & & & & 180 & & & & \\
\hline II & 145 & 50 & 850 & 680 & & 75 & 20 & & & & & 300 & & & & \\
\hline \multicolumn{17}{|c|}{ WITriid FIVE MILES } \\
\hline$I, V$ & 1350 & 450 & $d$ & 0 & & 105 & 20 & & & & & 420 & & & & \\
\hline$I, V I$ & 2815 & 940 & 300 & 240 & & 85 & 20 & & & & & 480 & & & & \\
\hline I I, I I I & 700 & 235 & 885 & 710 & & 100 & 20 & & & & & 480 & & & & \\
\hline II, IV & 1350 & 450 & 850 & 680 & & 115 & 20 & & & & & 420 & & & & \\
\hline \multicolumn{17}{|c|}{ WITHIN TEN MILES } \\
\hline$I, V, I X$ & 5310 & 1770 & d & 0 & & 115 & 20 & & & & & 540 & & & & \\
\hline$I, V I, X$ & 42240 & 14080 & 10300 & 8240 & & 115 & 20 & & & & & 720 & & & 300 & \\
\hline II , I I I, VII & 1510 & 505 & 885 & 710 & & 125 & 20 & & & & & 540 & & & & \\
\hline II $, I V, V I I I$ & 7265 & 2420 & 850 & 680 & & 100 & 20 & & & & & 480 & & & 60 & \\
\hline
\end{tabular}




\begin{tabular}{|c|c|c|c|c|c|c|c|c|c|c|c|c|c|c|c|c|}
\hline 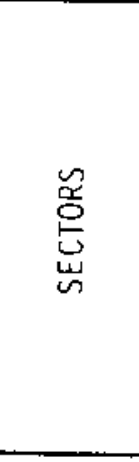 & 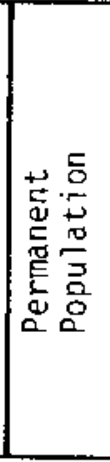 & 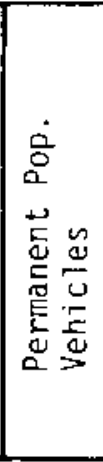 & 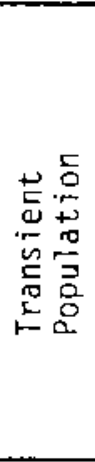 & 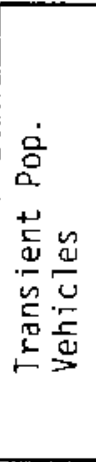 & 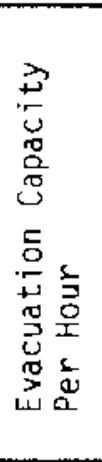 & 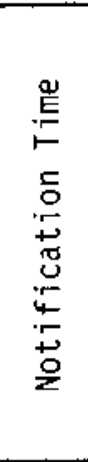 & 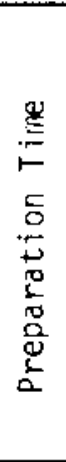 & 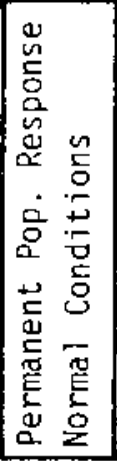 & 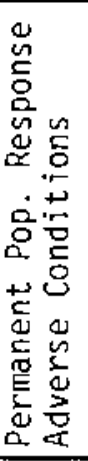 & 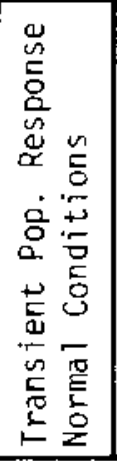 & 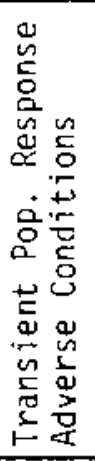 & 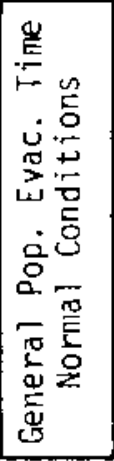 & 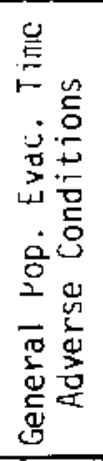 & 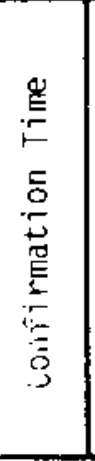 & 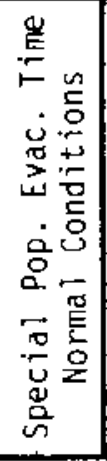 & 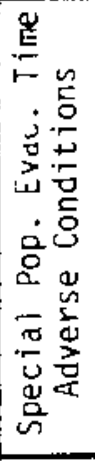 \\
\hline \multicolumn{17}{|c|}{ WITHIN TWO MILES } \\
\hline$R-G$ & 14 & 9 & 2234 & & & 35 & * & 3 & 6 & & & 38 & 41 & 16 & $\star *$ & $\star \star$ \\
\hline $\mathrm{H}-\mathrm{Q}$ & 87 & 56 & & & & 40 & $\star$ & 3 & 6 & & & 43 & 46 & 22 & $\star \star$ & $\star \star$ \\
\hline \multicolumn{17}{|c|}{ WIITHIN FIVE MILES } \\
\hline$R-C$ & 55 & 35 & & & & 35 & $\star$ & 9 & 24 & & & 44 & 59 & 30 & $\star \star$ & $\star \star$ \\
\hline$D-G$ & 35 & 22 & & & & 35 & $\star$ & 9 & 24 & & & 44 & 59 & 27 & $\star \star$ & $\star \star$ \\
\hline$M-Q$ & 478 & 306 & & & & 70 & $\star$ & 9 & 12 & & & 79 & 82 & 98 & $\star \star$ & $\star \star$ \\
\hline$H-L$ & 229 & 147 & & & & 55 & * & 11 & 28 & & & 66 & 83 & 132 & $\star \star$ & $\star \star$ \\
\hline \multicolumn{17}{|c|}{ WITHIN TEN MILES } \\
\hline$R-C$ & 303 & 194 & & & & 155 & * & 20 & 66 & & & 175 & 221 & 270 & $\star \star$ & $\star \star$ \\
\hline$D-G$ & 2491 & 1594 & & & & 200 & * & 20 & 66 & & & 220 & 266 & 343 & $\star \star$ & $\star \star$ \\
\hline$M-Q$ & 3674 & 2351 & & & & 200 & * & 20 & 64 & & & 220 & 264 & 652 & $\star \star$ & $\star \star$ \\
\hline$H-L$ & 1816 & 1162 & & & & 200 & * & 20 & 64 & & & 220 & 264 & 760 & $\star \star$ & $\star \star \star$ \\
\hline
\end{tabular}

* Preparation time included in warning time

$\star \star$ Less time than for the general population 
EVACUATION TIME ESTIMATES

Robinson *

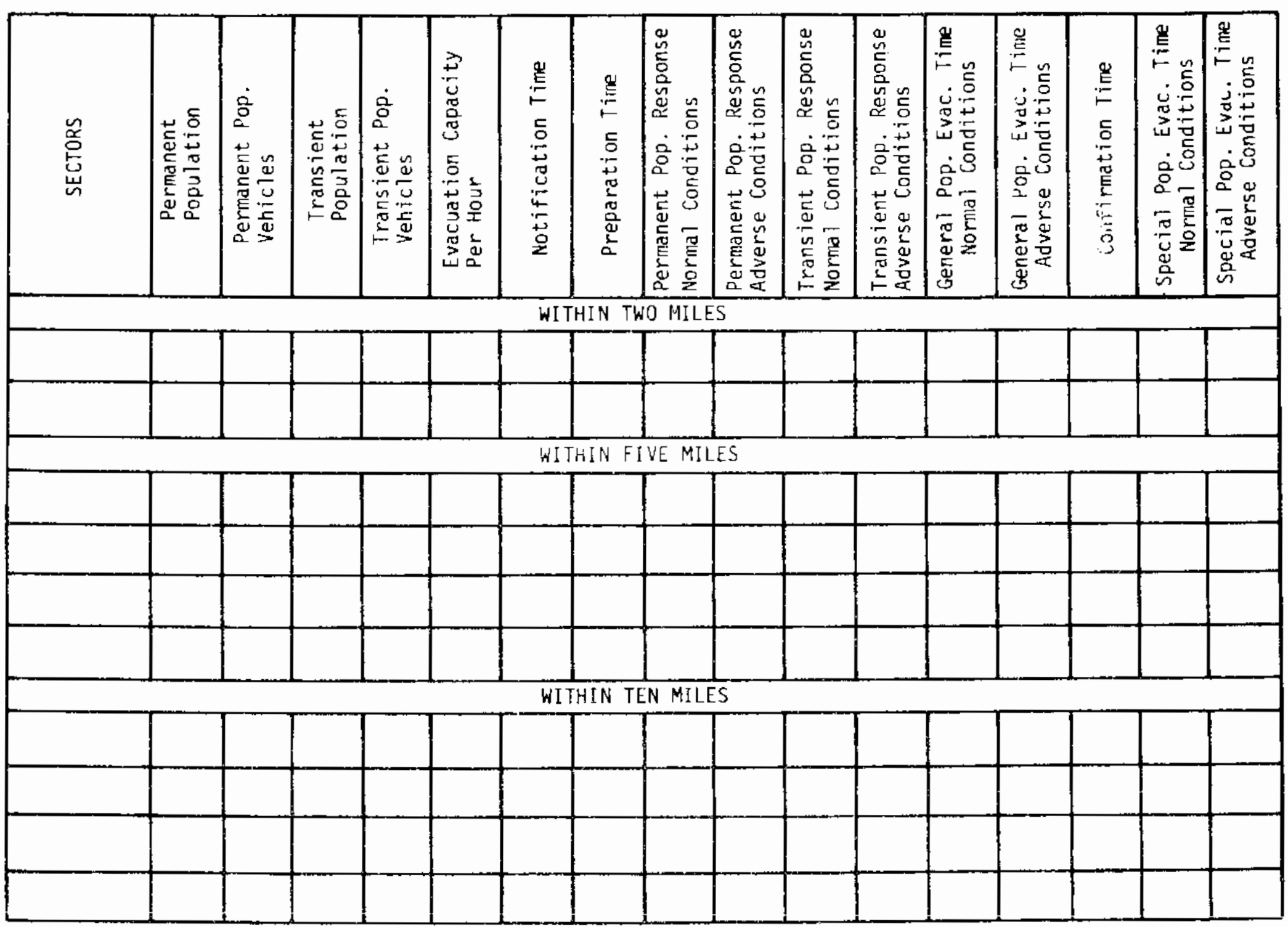

*No Response 
EVACUATION TIME ESTIMATES

Saint Lucie

\begin{tabular}{|c|c|c|c|c|c|c|c|c|c|c|c|c|c|c|c|c|}
\hline $\begin{array}{l}\text { 参 } \\
\text { 总 }\end{array}$ & 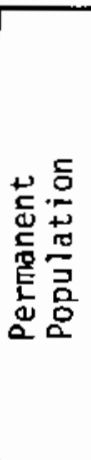 & 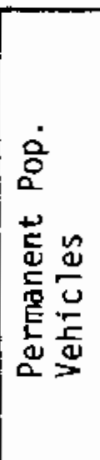 & 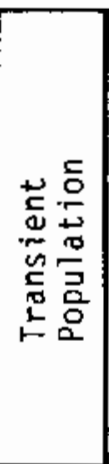 & 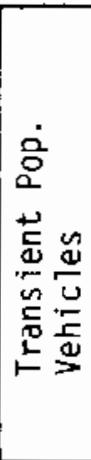 & 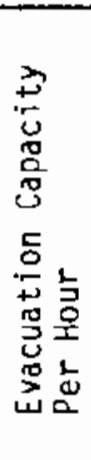 & 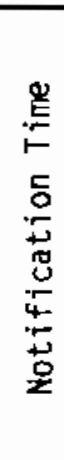 & 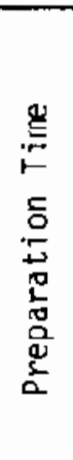 & 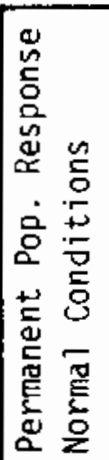 & 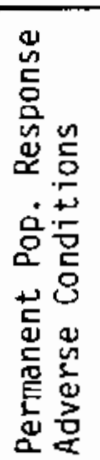 & 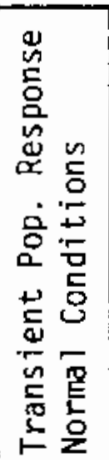 & 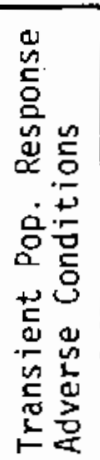 & 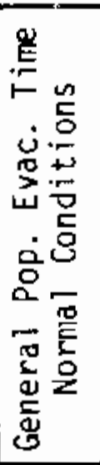 & 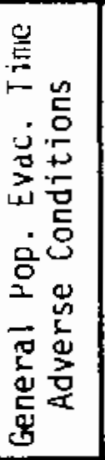 & 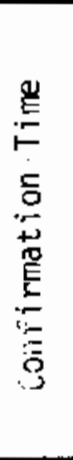 & 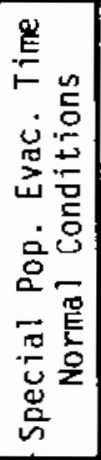 & 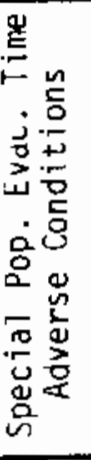 \\
\hline \multicolumn{17}{|c|}{ WITHIN TWO MILES } \\
\hline $\mathrm{N}-\mathrm{SSE}$ & & & & & & 30 & & & & & & 110 & 125 & 30 & & \\
\hline & & & & & & & & & & & & & & & & \\
\hline \multicolumn{17}{|c|}{ WITHIN FIVE MILES } \\
\hline $\mathrm{N}-\mathrm{NW}$ & & & & & & 30 & & & & & & 170 & 185 & 30 & & \\
\hline WNW-SW & & & & & & 30 & & & & & & 170 & 185 & 30 & & \\
\hline SSW-SE & & & & & & 30 & & & & & & 210 & 225 & 30 & & \\
\hline & & & & & & & & & & & & & & & & \\
\hline \multicolumn{17}{|c|}{ WI FHIN TEN MILES } \\
\hline $\mathrm{N}-\mathrm{NW}$ & & & & & & 30 & & & & & & 380 & 395 & 30 & & \\
\hline WNW-SW & & & & & & 30 & & & & & & 305 & 320 & 30 & & \\
\hline SSW-SE & & & & & & 30 & & & & & & 325 & 340 & 30 & & \\
\hline & & & & & & & & & & & & & & & & \\
\hline
\end{tabular}


EVACUAIIUN IIME ESTIMATES

Sa lem

$\stackrel{p}{1}$

\begin{tabular}{|c|c|c|c|c|c|c|c|c|c|c|c|c|c|c|c|c|}
\hline 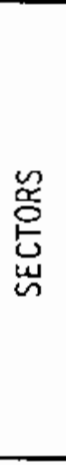 & 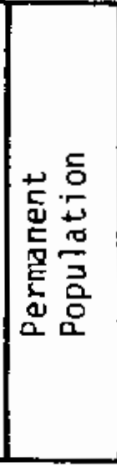 & 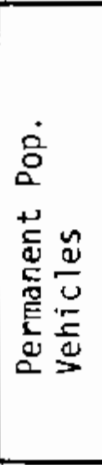 & 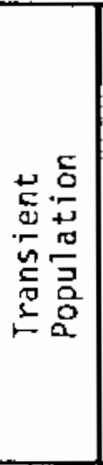 & 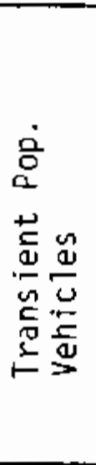 & 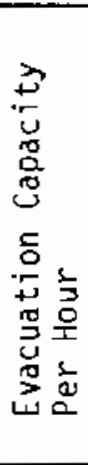 & 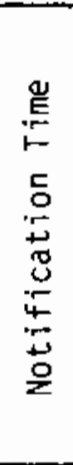 & 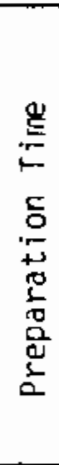 & 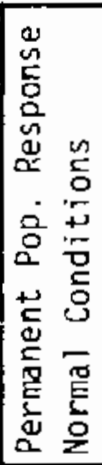 & 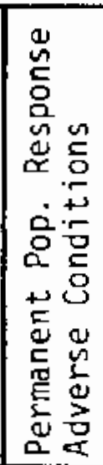 & 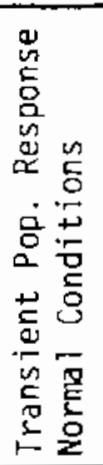 & 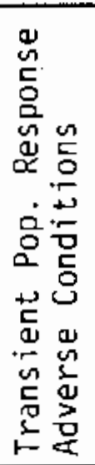 & 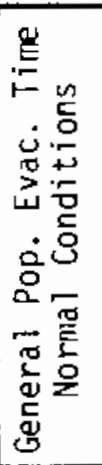 & 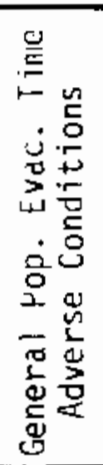 & 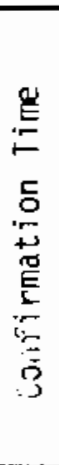 & 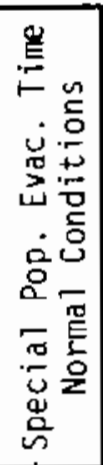 & 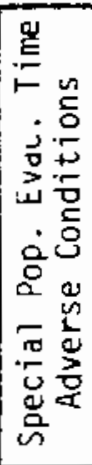 \\
\hline \multicolumn{17}{|c|}{ WITHIN TWO MILES } \\
\hline $\mathrm{E}$ & 0 & & & & & 0 & & 0 & 0 & & & 0 & 0 & & & \\
\hline$W$ & 0 & & & & & 0 & & 0 & 0 & & & 0 & 0 & & & \\
\hline \multicolumn{17}{|c|}{ WITHIN FIVE MILES } \\
\hline \multicolumn{17}{|c|}{468} \\
\hline \multicolumn{17}{|l|}{ SE } \\
\hline SW & 89 & & & & & 15 & & 105 & 165 & & & 120 & 180 & & & \\
\hline $\mathrm{NW}$ & 741 & & & & & 15 & & 105 & 165 & & & 120 & 180 & & & \\
\hline \multicolumn{17}{|c|}{ WITHIN TEN MILES } \\
\hline NE & 11451 & & & & & & & & & & & & & & & \\
\hline$\underline{S E}$ & 1574 & & & & & & & & & & & & & & & \\
\hline SW & 3112 & & & & & 30 & & 330 & 510 & & & 360 & 540 & & & \\
\hline $\mathrm{NW}$ & 7775 & & & & & 30 & & 330 & 510 & & & 360 & 540 & & & \\
\hline
\end{tabular}


EVACUATION TIME ESTIMATES

San Onofre

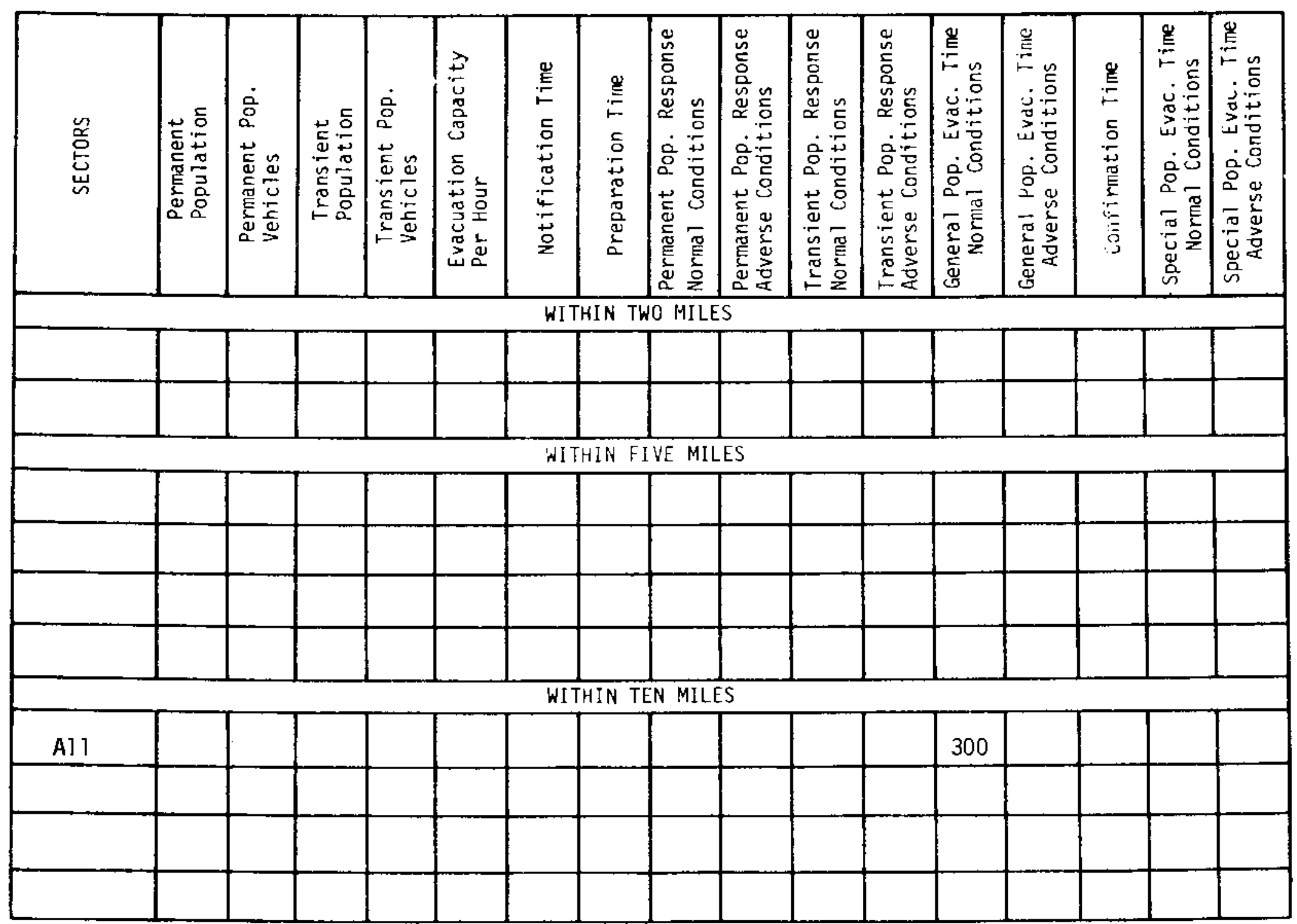




\begin{tabular}{|c|c|c|c|c|c|c|c|c|c|c|c|c|c|c|c|c|}
\hline $\begin{array}{l}\text { 苍 } \\
\text { 总 } \\
\text { 出 }\end{array}$ & 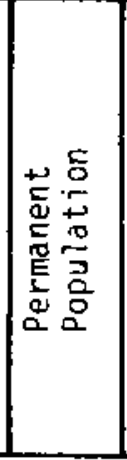 & 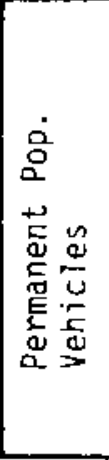 & 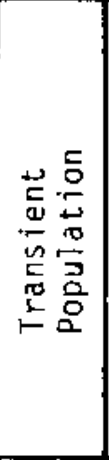 & 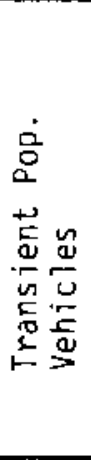 & 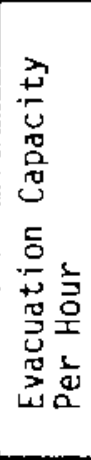 & 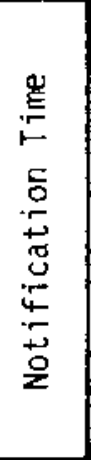 & 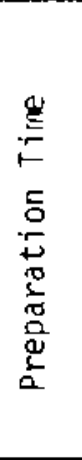 & 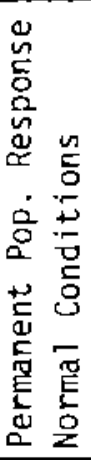 & 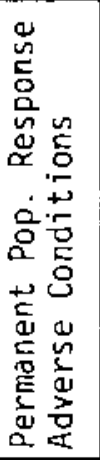 & 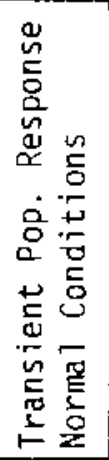 & 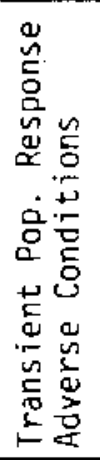 & 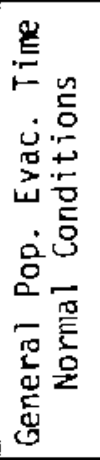 & 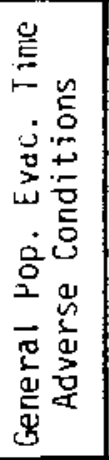 & 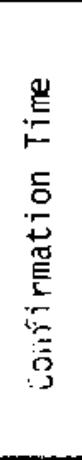 & 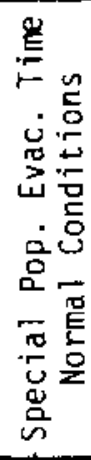 & 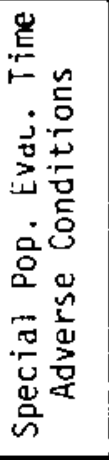 \\
\hline \multicolumn{17}{|c|}{ WITHIN TWO MILES } \\
\hline$A \& D$ & 774 & 219 & & & & 15 & 30 & 9 & 39 & & & 54 & 84 & 120 & 90 & 110 \\
\hline$B \& C$ & 1560 & 1452 & & & & 15 & 30 & 18 & 48 & & & 63 & 93 & 120 & 115 & 135 \\
\hline \multicolumn{17}{|c|}{ WITHIN FIVE MILES } \\
\hline A & 4833 & 1372 & & & & 15 & 30 & 31 & 61 & & & 76 & 96 & 120 & 115 & 135 \\
\hline B & 1950 & 553 & & & & 15 & 30 & 18 & 48 & & & 63 & 93 & 120 & 115 & 135 \\
\hline C & 3341 & 948 & & & & 15 & 30 & 24 & 54 & & & 69 & 99 & 120 & 115 & 135 \\
\hline D & 3173 & 1039 & & & & 15 & 30 & 26 & 56 & & & 71 & 91 & 120 & 115 & 135 \\
\hline \multicolumn{17}{|c|}{ WITHIN TEN MILES } \\
\hline A & 6840 & 1941 & & & & 15 & 30 & 88 & 118 & & & 133 & 163 & 180 & 160 & 190 \\
\hline$B$ & 2730 & 775 & & & & 15 & 30 & 28 & 58 & & & 73 & 103 & 180 & 160 & 190 \\
\hline C & 8900 & 2526 & & & & 15 & 30 & 29 & 59 & & & 74 & 104 & 180 & 160 & 190 \\
\hline$D$ & 19020 & 5398 & & & & 15 & 30 & 82 & 112 & & & 127 & 157 & 180 & 160 & 190 \\
\hline
\end{tabular}




\begin{tabular}{|c|c|c|c|c|c|c|c|c|c|c|c|c|c|c|c|c|}
\hline $\begin{array}{l}\text { 参 } \\
\text { 总 }\end{array}$ & 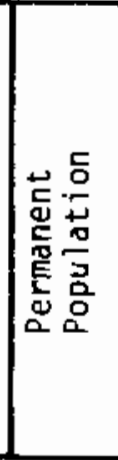 & 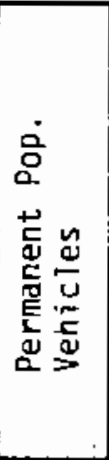 & 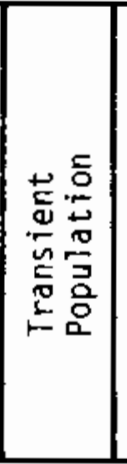 & 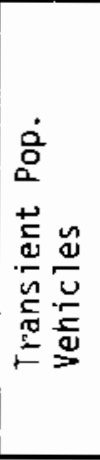 & 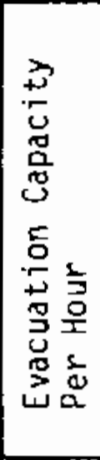 & 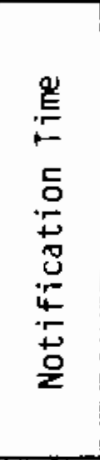 & 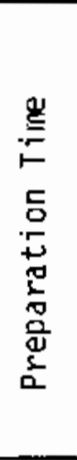 & 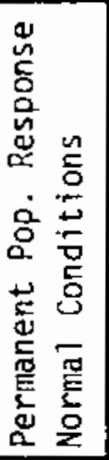 & 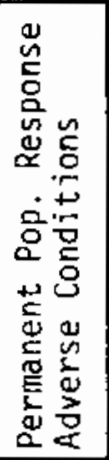 & 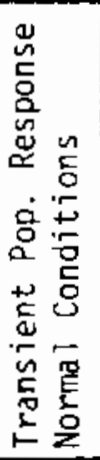 & 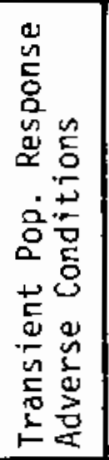 & 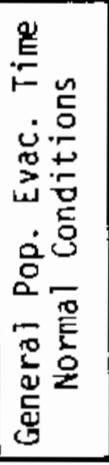 & 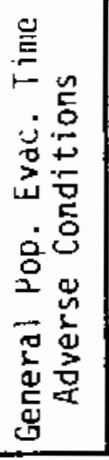 & 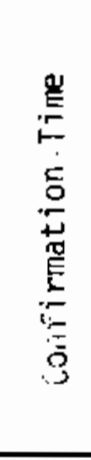 & 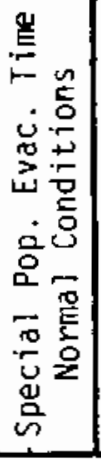 & 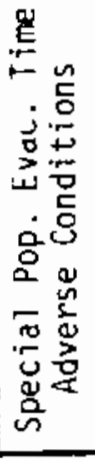 \\
\hline \multicolumn{17}{|c|}{ WITHIN TWO MILES } \\
\hline $\mathrm{A}-\mathrm{H}$ & 0 & & 0 & & & 0 & & 0 & 0 & 0 & 0 & 0 & 0 & 0 & 0 & 0 \\
\hline$J-R$ & 24 & & 0 & & & 30 & & 60 & 90 & 0 & 0 & 90 & 120 & 60 & 0 & 0 \\
\hline \multicolumn{17}{|c|}{ WITHIN FIVE MILES } \\
\hline$A-0$ & 1074 & & 0 & & & 135 & & 195 & 360 & 0 & 0 & 330 & 495 & 70 & 0 & 0 \\
\hline E-H & 3624 & & 0 & & & 90 & & 120 & 240 & 0 & 0 & 210 & 330 & 120 & 180 & 240 \\
\hline$J-M$ & 664 & & 0 & & & 90 & & 120 & 180 & 0 & 0 & 210 & 270 & 120 & 0 & 0 \\
\hline $\mathrm{N}-\mathrm{R}$ & 138 & & of & & & 60 & & 60 & 120 & 0 & 0 & 120 & 180 & 60 & 0 & 0 \\
\hline \multicolumn{17}{|c|}{ WITHIN TEN MILES } \\
\hline$A-0$ & 27940 & & 10000 & & & 240 & & 300 & 540 & 80 & 140 & 540 & 780 & 480 & 240 & 300 \\
\hline $\mathrm{E}-\mathrm{H}$ & 44736 & & 0 & & & 120 & & 240 & 300 & 0 & 0 & 360 & 420 & 240 & 0 & 0 \\
\hline$J-M$ & 4548 & & 0 & & & 240 & & 360 & 450 & 0 & 0 & 600 & 690 & 360 & 0 & 0 \\
\hline $\mathrm{N}-\mathrm{R}$ & 5283 & & 0 & & & 120 & & 270 & 360 & 0 & 0 & 390 & 480 & 240 & 0 & 0 \\
\hline
\end{tabular}


FVACILATION TIME ESTIMATES

Three Mile Island

\begin{tabular}{|c|c|c|c|c|c|c|c|c|c|c|c|c|c|c|c|c|}
\hline 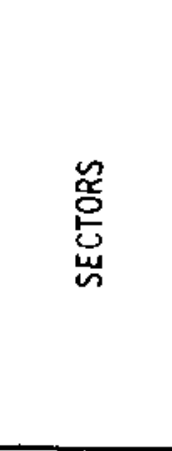 & 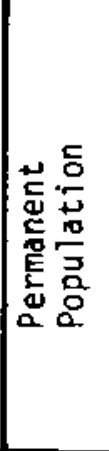 & 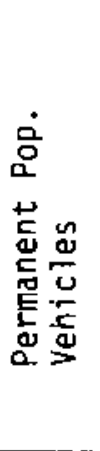 & 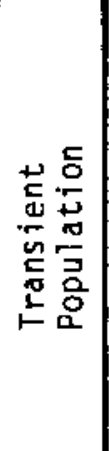 & 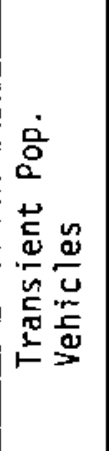 & 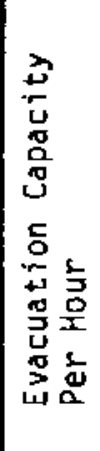 & 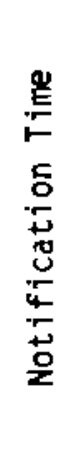 & 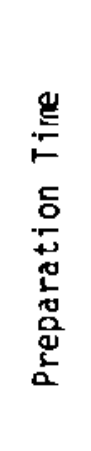 & 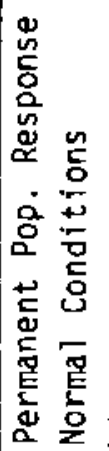 & 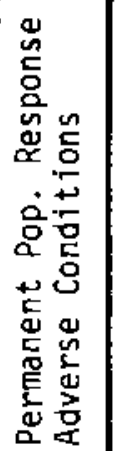 & 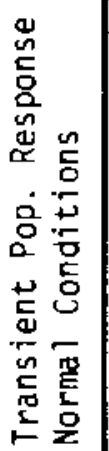 & 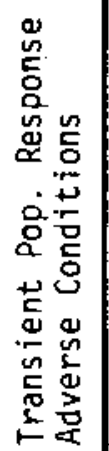 & 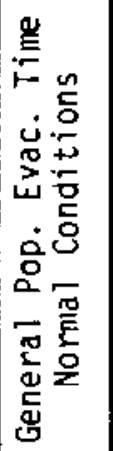 & 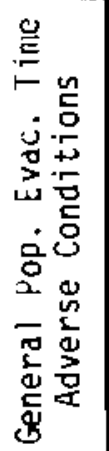 & 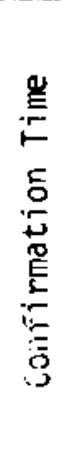 & 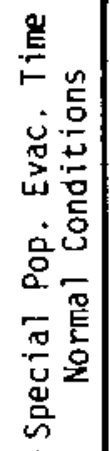 & 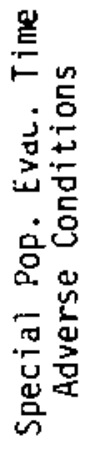 \\
\hline \multicolumn{17}{|c|}{ WITHIN TWO HILES } \\
\hline $\begin{array}{l}\text { Dauphin } \\
\text { County }\end{array}$ & & & & & & & 60 & 60 & 120 & & & 120 & 180 & & & \\
\hline \multirow{2}{*}{\multicolumn{16}{|c|}{ WITHIN FIVE MILES }} & \\
\hline Dauphin & & & & & & & & & & & & & & & & \\
\hline County & & & & & & & 180 & 240 & 480 & & & 420 & 660 & & 300 & 600 \\
\hline & & & & & & & & & & & & & & & & \\
\hline & & & & & & & & & & & & & & & & \\
\hline & & & & & & & & & & & & & & & & \\
\hline \multicolumn{17}{|c|}{ WITHIN TEN MILES } \\
\hline $\begin{array}{l}\text { Dauphin } \\
\text { County }\end{array}$ & & & & & & & 360 & 420 & 1260 & & & 780 & 1620 & & 1440 & 2880 \\
\hline & & & & & & & & & & & & & & & & \\
\hline & & & & & & & & & & & & & & & & \\
\hline & & & & & & & & & & & & & & & & \\
\hline
\end{tabular}




\begin{tabular}{|c|c|c|c|c|c|c|c|c|c|c|c|c|c|c|c|c|}
\hline $\begin{array}{l}\text { ్ㅗㅇ } \\
\text { 岕 }\end{array}$ & 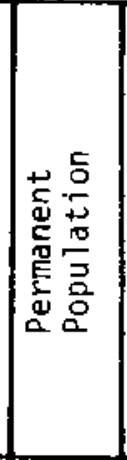 & 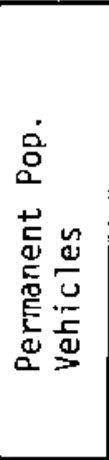 & 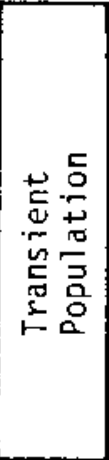 & 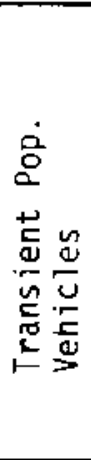 & 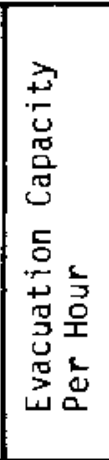 & 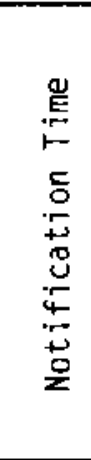 & 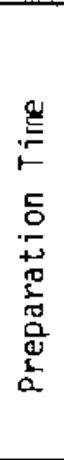 & 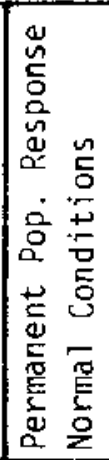 & 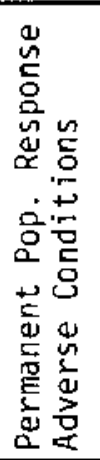 & 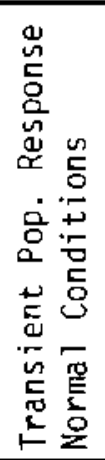 & 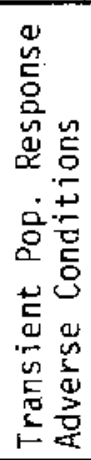 & 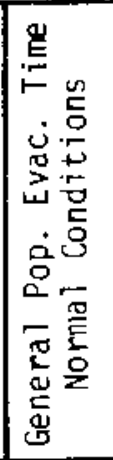 & 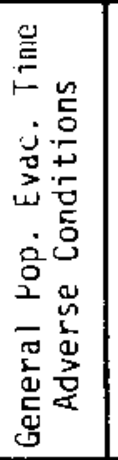 & 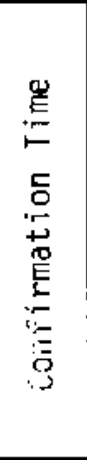 & 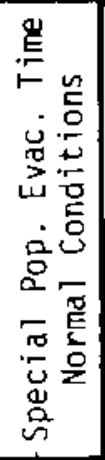 & 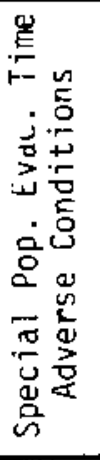 \\
\hline \multicolumn{17}{|c|}{ WITHIN TWO MILES } \\
\hline W-ENE & 606 & 205 & & & 2100 & 57 & & 41 & 61 & & & 98 & 118 & 60 & & \\
\hline E-WSW & 852 & 227 & & & 2100 & 45 & & 37 & 56 & & & 82 & 101 & 60 & & \\
\hline \multicolumn{17}{|c|}{ WITTIN FIVE MILES } \\
\hline WNW-N & 3266 & 1179 & & & 2100 & 120 & & 103 & 123 & & & 223 & 243 & 60 & & \\
\hline NNE-E & 1637 & 623 & & & 2400 & 82 & & 56 & 71 & & & 138 & 153 & 60 & & \\
\hline ESE-S & 2245 & 950 & & & 2100 & 106 & & 52 & 70 & & & 158 & 176 & 60 & & \\
\hline SSW-W & 1171 & 562 & & & 1800 & 43 & & 56 & 76 & & & 99 & 116 & 60 & & \\
\hline \multicolumn{17}{|c|}{ WITHIN TEN MILES } \\
\hline WNW-N & 54040 & 1810 & & & 3600 & 86 & & 255 & 272 & & & 341 & 358 & 120 & 220 & 281 \\
\hline NNE-E & 3850 & 1363 & & & 2400 & 277 & & 82 & 120 & & & 359 & 397 & 120 & & \\
\hline ESE-S & 4522 & 1611 & & & 2100 & 103 & & 73 & 92 & & & 176 & 195 & 120. & & \\
\hline SSW-W & 2214 & 912 & & & 2400 & 66 & & 62 & 81 & & & 128 & 147 & 120 & & \\
\hline
\end{tabular}


Turkey Point

\begin{tabular}{|c|c|c|c|c|c|c|c|c|c|c|c|c|c|c|c|c|}
\hline $\begin{array}{l}\text { 哭 } \\
\text { 总 }\end{array}$ & 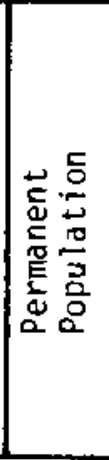 & 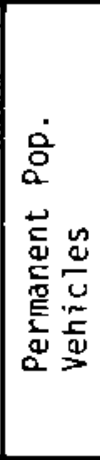 & 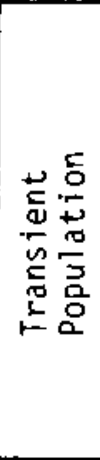 & 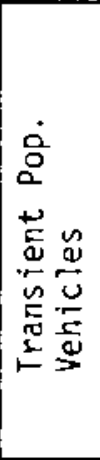 & 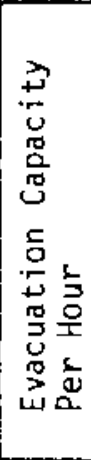 & 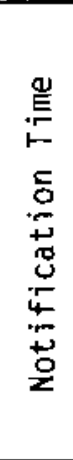 & 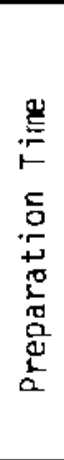 & 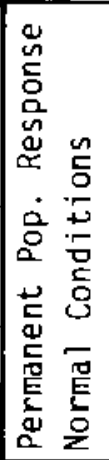 & 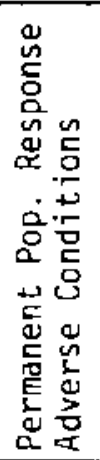 & 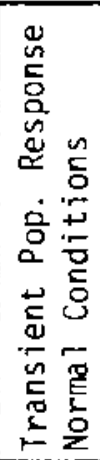 & 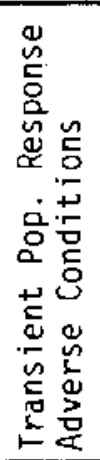 & 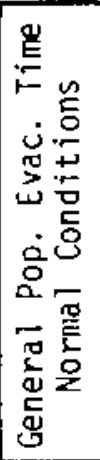 & 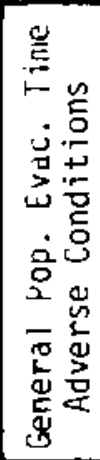 & 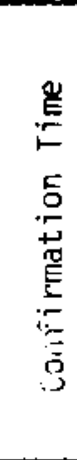 & 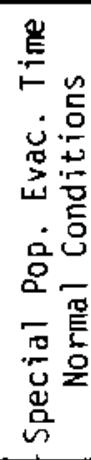 & 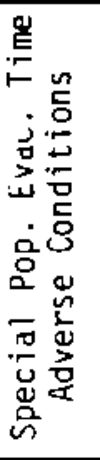 \\
\hline \multicolumn{17}{|c|}{ WITHIN TWO MILES } \\
\hline$N-S S E$ & & & & & & 30 & & & & & & 90 & 105 & 60 & & \\
\hline \multicolumn{17}{|c|}{ WITHIN FIVE MILES } \\
\hline $\mathrm{N}-\mathrm{NW}$ & & & & & & 30 & & & & & & 100 & 115 & 60 & & \\
\hline WNW-SW & & & & & & 30 & & & & & & 95 & 110 & 60 & & \\
\hline SSW-SE & & & & & & 30 & & & & & & 90 & 105 & 60 & & \\
\hline & & & & & & & & & & & & & & & & \\
\hline \multicolumn{17}{|c|}{ WITHIN TEN MILES } \\
\hline $\mathrm{N}-\mathrm{NW}$ & & & & & & 30 & & & & & & 255 & 270 & 60 & & \\
\hline WNW-SW & & & & & & 30 & & & & & & 365 & 380 & 60 & & \\
\hline SSW-SE & & & & & & 30 & & & & & & 90 & 105 & 60 & & \\
\hline & & & & & & & & & & & & & & & & \\
\hline
\end{tabular}




\begin{tabular}{|c|c|c|c|c|c|c|c|c|c|c|c|c|c|c|c|c|}
\hline $\begin{array}{l}\text { 号 } \\
\text { 吕 }\end{array}$ & 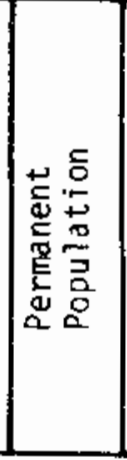 & 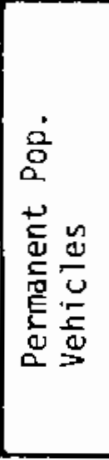 & 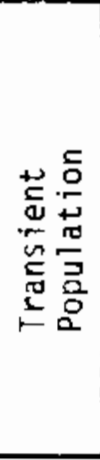 & 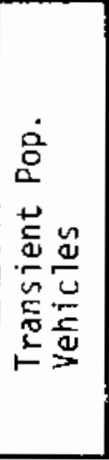 & 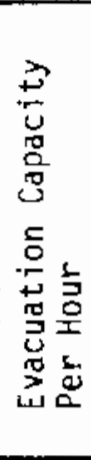 & 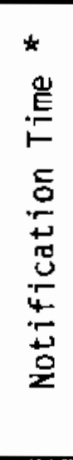 & 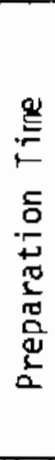 & 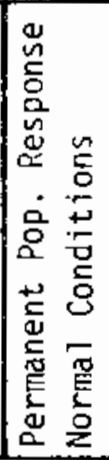 & 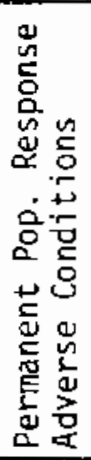 & 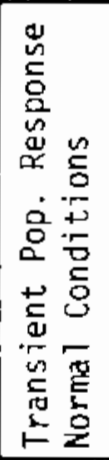 & 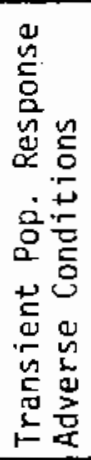 & 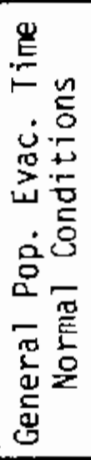 & 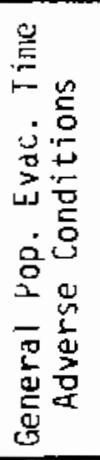 & 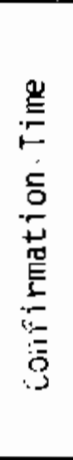 & 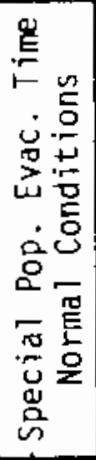 & 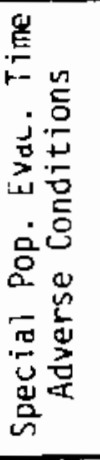 \\
\hline \multicolumn{17}{|c|}{ WI THIN TWO MILES } \\
\hline$P-E$ & 2060 & 687 & & & & $\frac{35}{45}$ & & & & & & & & & 69 & 78 \\
\hline$F-N$ & $\sigma$ & 218 & & & & $\frac{35}{45}$ & & & & & & & & & 70 & 80 \\
\hline \multicolumn{17}{|c|}{ WITHIN FIVE MILES } \\
\hline$C-F$ & 2240 & 1160 & & & & $\frac{35}{45}$ & & & & & & & & & 76 & 92 \\
\hline$G-K$ & 850 & 460 & & & & $\frac{35}{45}$ & & & & & & & & & 74 & 84 \\
\hline L-P & 500 & 360 & & & & $\frac{35}{45}$ & & 18 & 36 & & & 53 & 81 & & & \\
\hline$Q-B$ & 3400 & 1640 & & 1200 & & $\frac{35}{45}$ & & & & & & & & & 70 & 85 \\
\hline \multicolumn{17}{|c|}{ WITHIN TEN MILES } \\
\hline$c-F$ & 3100 & 2360 & & & & $\frac{35}{45}$ & & 32 & 64 & & & 67 & 109 & & & \\
\hline G-K & 6800 & 3180 & & & & $\frac{35}{45}$ & & & & & & & & & 111 & 162 \\
\hline L-P & 1150 & 820 & & & & $\frac{35}{45}$ & & 26 & 52 & & & 61 & 97 & & & \\
\hline$Q-B$ & 14450 & 6106 & & 1200 & & $\frac{35}{45}$ & & & & & & & & & 180 & 180 \\
\hline
\end{tabular}

Note: Evacuation times only reported for controlling condition, either general population evacuation or special facilities evacuation

* Normal (Top)/Adverse (Bottom) 


\begin{tabular}{|c|c|c|c|c|c|c|c|c|c|c|c|c|c|c|c|c|}
\hline 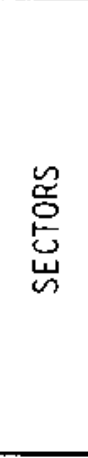 & 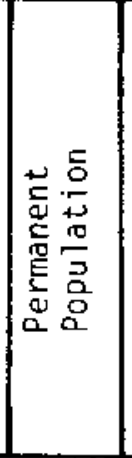 & 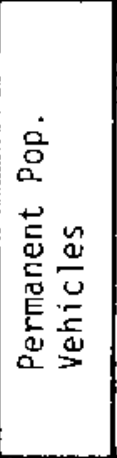 & 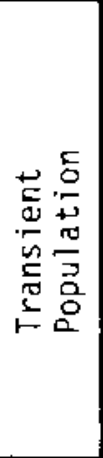 & 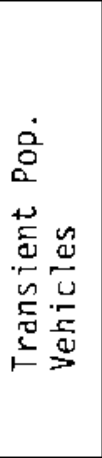 & 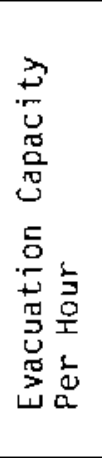 & 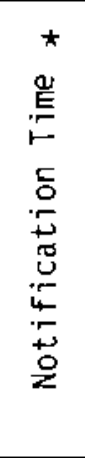 & 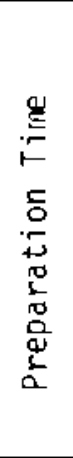 & 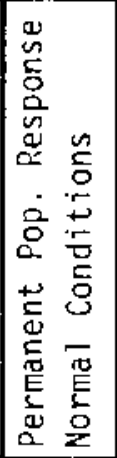 & 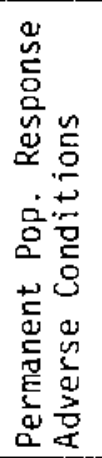 & 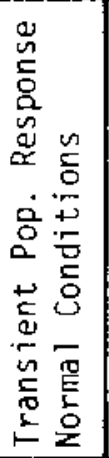 & 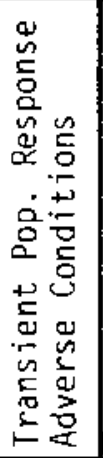 & 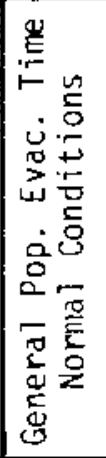 & 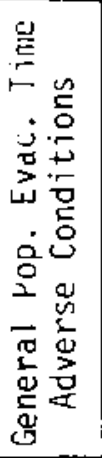 & 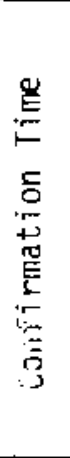 & 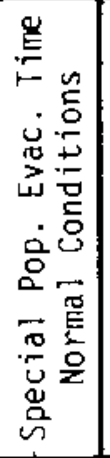 & 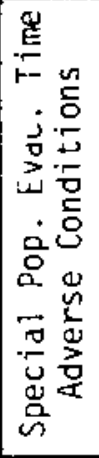 \\
\hline \multicolumn{17}{|c|}{ WITHIN TWO MILES } \\
\hline$Q-F$ & & 9 & & & 2790 & $\frac{35}{45}$ & & 7 & 13 & & & & & & & \\
\hline G-P & 225 & 90 & & & 2790 & $\frac{35}{45}$ & & 7 & 13 & & & & & & & \\
\hline \multicolumn{17}{|c|}{ WITHIN FIVE MILES } \\
\hline$B-E$ & 425 & 192 & & & 2790 & $\frac{35}{45}$ & & 13 & 26 & & & & & & & \\
\hline $\mathrm{F}-\mathrm{J}$ & 315 & 154 & & & 2790 & $\frac{35}{45}$ & & 18 & 37 & & & & & & & \\
\hline$K-N$ & 240 & 114 & & & 2790 & $\frac{35}{45}$ & & 19 & 37 & & & & & & & \\
\hline$P-A$ & 530 & 114 & & & 2790 & $\frac{35}{45}$ & & 21 & 41 & & & & & & & \\
\hline \multicolumn{17}{|c|}{ WITHIN TEN MILES } \\
\hline$B-E$ & 1650 & 660 & & & 2790 & $\frac{35}{45}$ & & 41 & 82 & & & & & & & \\
\hline $\mathrm{F}-\mathrm{J}$ & 1400 & 560 & & & 2790 & $\frac{35}{45}$ & & 37 & 75 & & & & & & & \\
\hline $\mathrm{K}-\mathrm{N}$ & 13175 & 5270 & & & 2790 & $\frac{35}{45}$ & & 55 & 110 & & & & & & & \\
\hline $\mathrm{P}-\mathrm{A}$ & 700 & 280 & & & 2790 & $\frac{35}{45}$ & & 32 & 64 & & & & & & & \\
\hline
\end{tabular}

* Normal Conditions Top Figure - Adverse Conditions Bottom Figure 


\begin{tabular}{|c|c|c|c|c|c|c|c|c|c|c|c|c|c|c|c|c|}
\hline 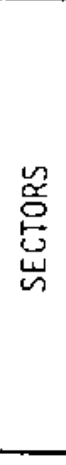 & 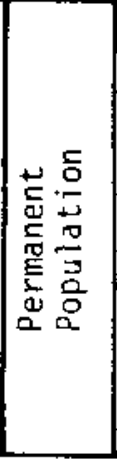 & 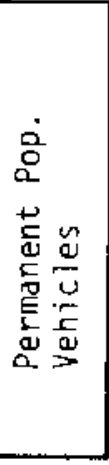 & 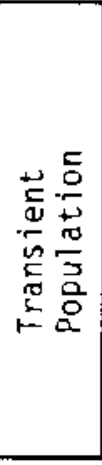 & 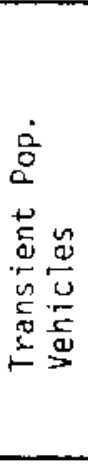 & 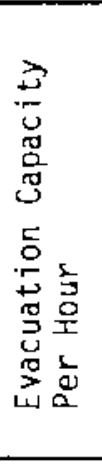 & 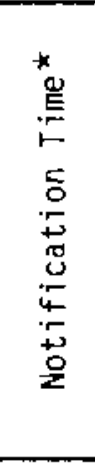 & 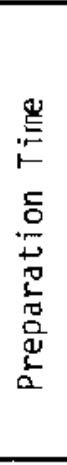 & 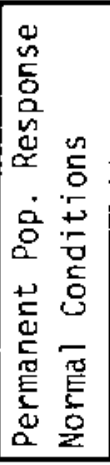 & 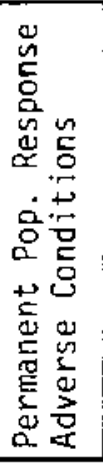 & 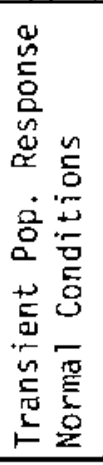 & 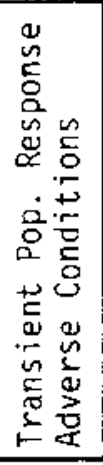 & 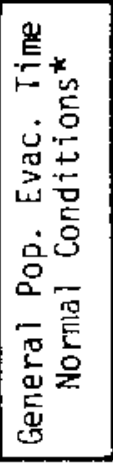 & 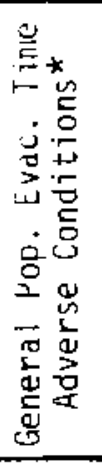 & 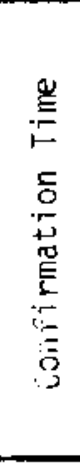 & 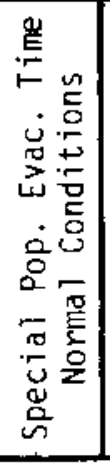 & 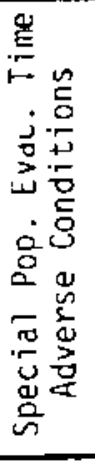 \\
\hline \multicolumn{17}{|c|}{ WITHIN TWO MILES } \\
\hline$E$ & 609 & 185 & 25 & 20 & & $\frac{15}{65}$ & & & & & & $\frac{60}{110}$ & $\frac{65}{115}$ & 65 & & \\
\hline$W$ & 359 & 109 & 80 & 64 & & $\frac{15}{65}$ & & & & & & $\frac{60}{110}$ & $\frac{65}{115}$ & 65 & & \\
\hline \multicolumn{17}{|c|}{ WITHIN FIVE MILES } \\
\hline N & 1876 & 510 & 25 & 20 & & $\frac{15}{80}$ & & & & & & $\frac{100}{160}$ & $\frac{125}{185}$ & 80 & & \\
\hline E & 1417 & 431 & 25 & 20 & & $\frac{15}{80}$ & & & & & & $\frac{100}{160}$ & $\frac{125}{185}$ & 80 & & \\
\hline$S$ & 858 & 260 & 80 & 64 & & $\frac{15}{120}$ & & & & & & $\frac{100}{205}$ & $\frac{125}{230}$ & 120 & & \\
\hline$W$ & 1246 & 379 & 80 & 64 & & $\frac{15}{70}$ & & & & & & $\frac{100}{155}$ & $\frac{125}{180}$ & 70 & & \\
\hline \multicolumn{17}{|c|}{ WITHIN TEN MILES } \\
\hline$N$ & 12788 & 3887 & 475 & 380 & & $\frac{45}{140}$ & & & & & & $\frac{175}{265}$ & $\frac{235}{325}$ & 140 & & \\
\hline$E$ & 6515 & 1980 & 25 & 20 & & $\frac{45}{280}$ & & & & & & $\frac{155}{385}$ & $\frac{205}{430}$ & 280 & & \\
\hline$S$ & 2303 & 700 & 580 & 189 & & $\frac{45}{300}$ & & & & & & $\frac{155}{410}$ & $\frac{205}{455}$ & 300 & & \\
\hline$W$ & 4774 & 1451 & 580 & 189 & & $\frac{45}{110}$ & & & & & & $\frac{155}{216}$ & $\frac{205}{265}$ & 110 & & \\
\hline
\end{tabular}

\footnotetext{
* Prompt (Top)/Without Prompt Notification System (Bottom)
} 


\begin{tabular}{|c|c|c|c|c|c|c|c|c|c|c|c|c|c|c|c|c|}
\hline 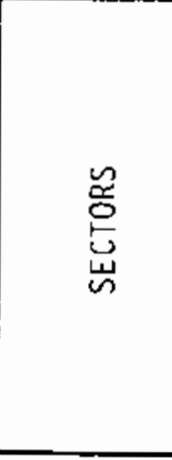 & 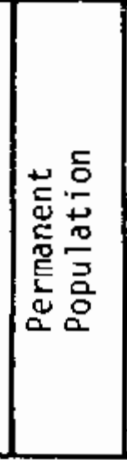 & 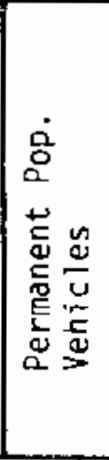 & 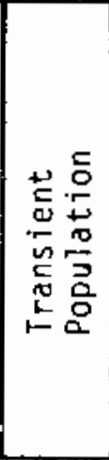 & 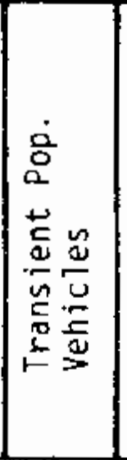 & 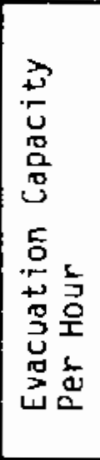 & 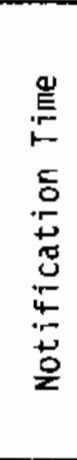 & 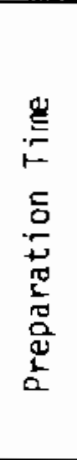 & 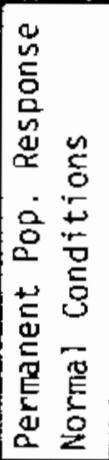 & 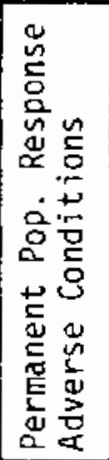 & 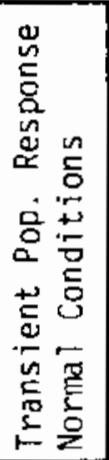 & 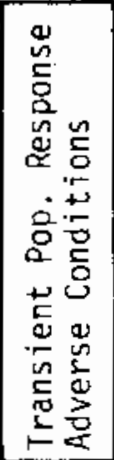 & 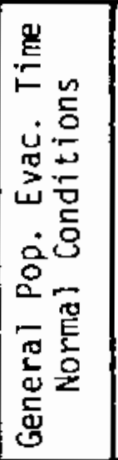 & 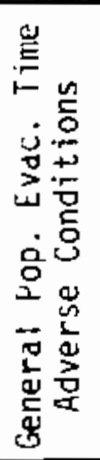 & 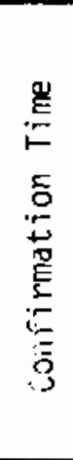 & 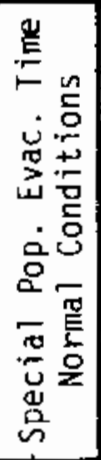 & 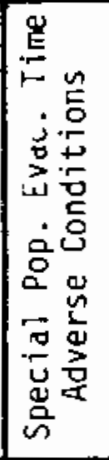 \\
\hline \multicolumn{17}{|c|}{ WITHIN TWO MILES } \\
\hline I & 28700 & 8735 & 435 & 350 & & 30 & 20 & & & & & 300 & & & 720 & \\
\hline \multirow{2}{*}{\multicolumn{17}{|c|}{ WITHIN FIVE MILES }} \\
\hline & & & & & & & & & & & & & & & & \\
\hline$I, I I$ & 38355 & 11750 & 660 & 530 & & 30 & 20 & & & & & 360 & & & 720 & \\
\hline I, III & 49100 & 15640 & 6140 & 4915 & & 45 & 20 & & & & & 360 & & & 720 & \\
\hline & & & & & & & & & & & & & & & & \\
\hline & & & & & & & & & & & & & & & & \\
\hline \multicolumn{17}{|c|}{ WITHIN TEN MILES } \\
\hline I, II, IV & 11870 & 3853 & 27095 & 21700 & & 45 & 20 & & & & & 1260 & & & 720 & \\
\hline $\mathrm{I}, \mathrm{I} I \mathrm{I}, \mathrm{V}$ & 150000 & 4879 & 39090 & 31275 & & 60 & 20 & & & & & 480 & & & 1440 & \\
\hline & & & & & & & & & & & & & & & & \\
\hline & & & & & & & & & & & & & & & & \\
\hline
\end{tabular}


APPENDIX C

DEFINITION OF EVACUATION TIME COMPONENTS

C-1 

The term "evacuation time" is used by different analysts to represent different components of the time between detection of an incident and the completion of an evacuation. For the purpose of this study, evacuation time is divided into several components. This allows comparison of the same components among the various sites.

The components of evacuation time are defined as follows:

$\underline{\text { Notification Time }}=$ The time required to get the evacuation notification to all individuals in the specified area.

Preparation Time $=$ The time required for individuals to prepare to evacuate the specified area.

$\underline{\text { Response Time }}=$ The time required for all individuals to physically move out of an area. This time component is shown separately for permanent residents (PPR) and for transients (TPR). Furthermore, separate estimates are also possible for normal (NC) and adverse conditions ( $A C$ ). The four possible response time components are: PPRNC, PPRAC, TPRNC and TPRAC.

General Population Evacuation Time = The sum of notification, preparation and response (both permanent and transient populations) times. Separate totals would be made for normal (GPTNC) and adverse (GPTAC) weather conditions. In most cases the total is an arithmetic sum of component times; in a few cases the total is a statistical sum of component distributions.

Special Population Respone Time = The time required to evacuate institutions and the time required by other special conditions that are largely independent of general population evacuation times. An example of a special condition is the evacuation time required for recreational 
pleasure boats at sites adjacent to bodies of water. Separate estimates are possible for normal (SPRNC) and adverse (SPRAC) weather conditions. In addition to the above evacuation time components, confirmation time estimates have also been required.

Confirmation Time $=$ The period of time required to verify that the affected population has departed. It may occur concurrentiy or subsequent to evacuation. It is not considered an evacuation time component. 


\section{APPENDIX D \\ EVALUATION FORMS FOR FEMA ASSESSMENTS}

D-1 



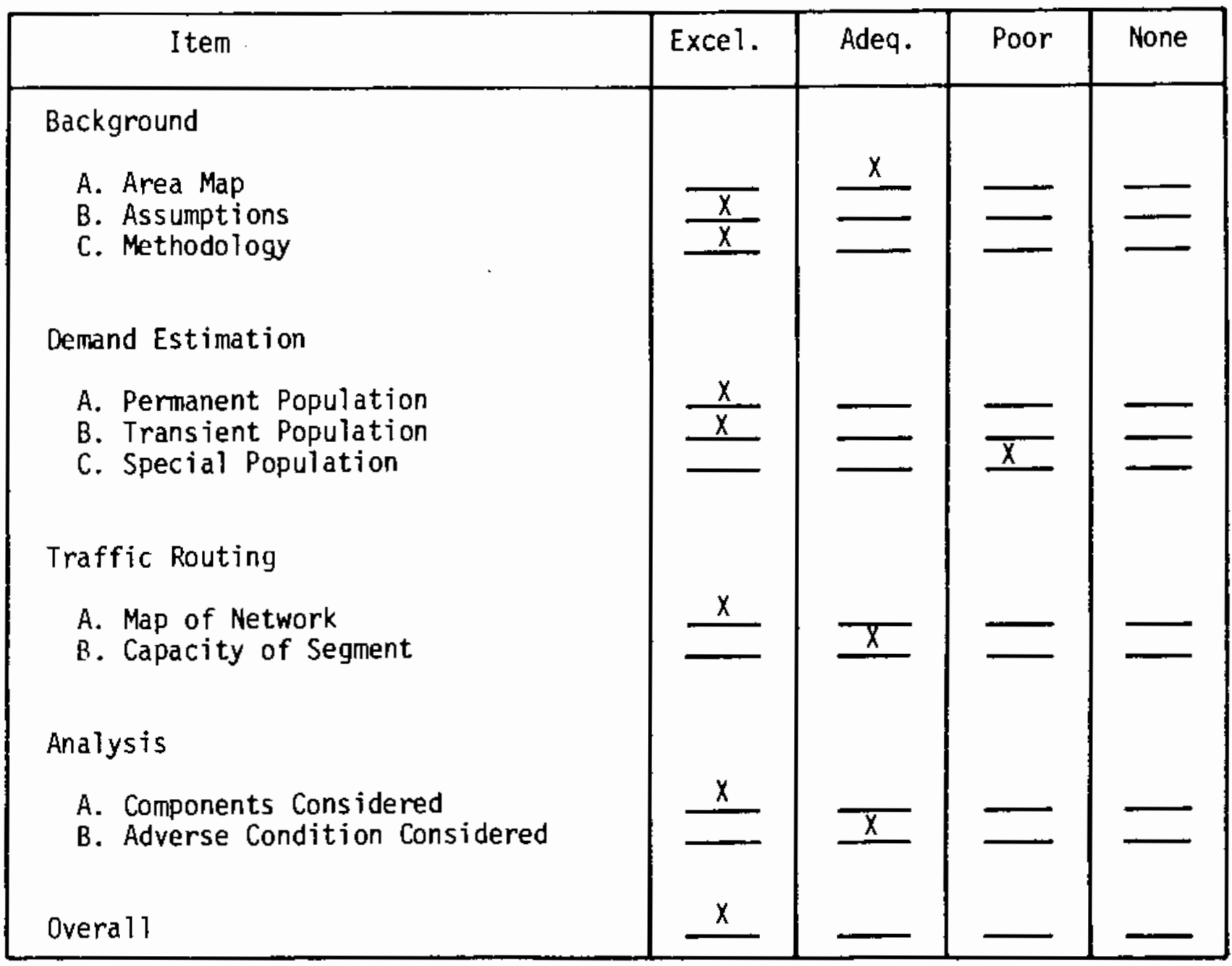

*Estimate prepared by FEMA contractor. 


\section{EVALUATION OF EVACUATION TIME ESTIMATE}

\section{Indian Point*}

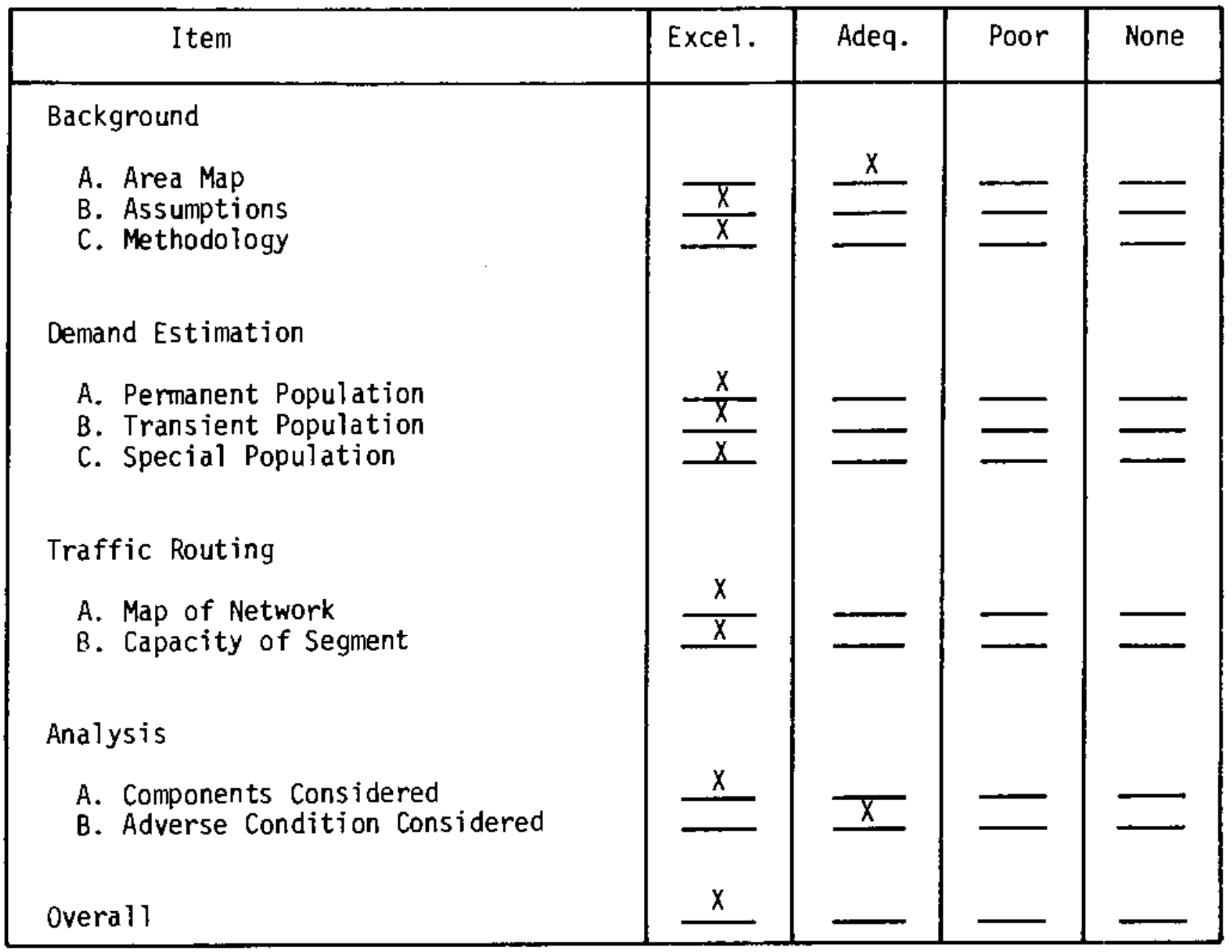

\#Estimate prepared by FEMA contractor. 


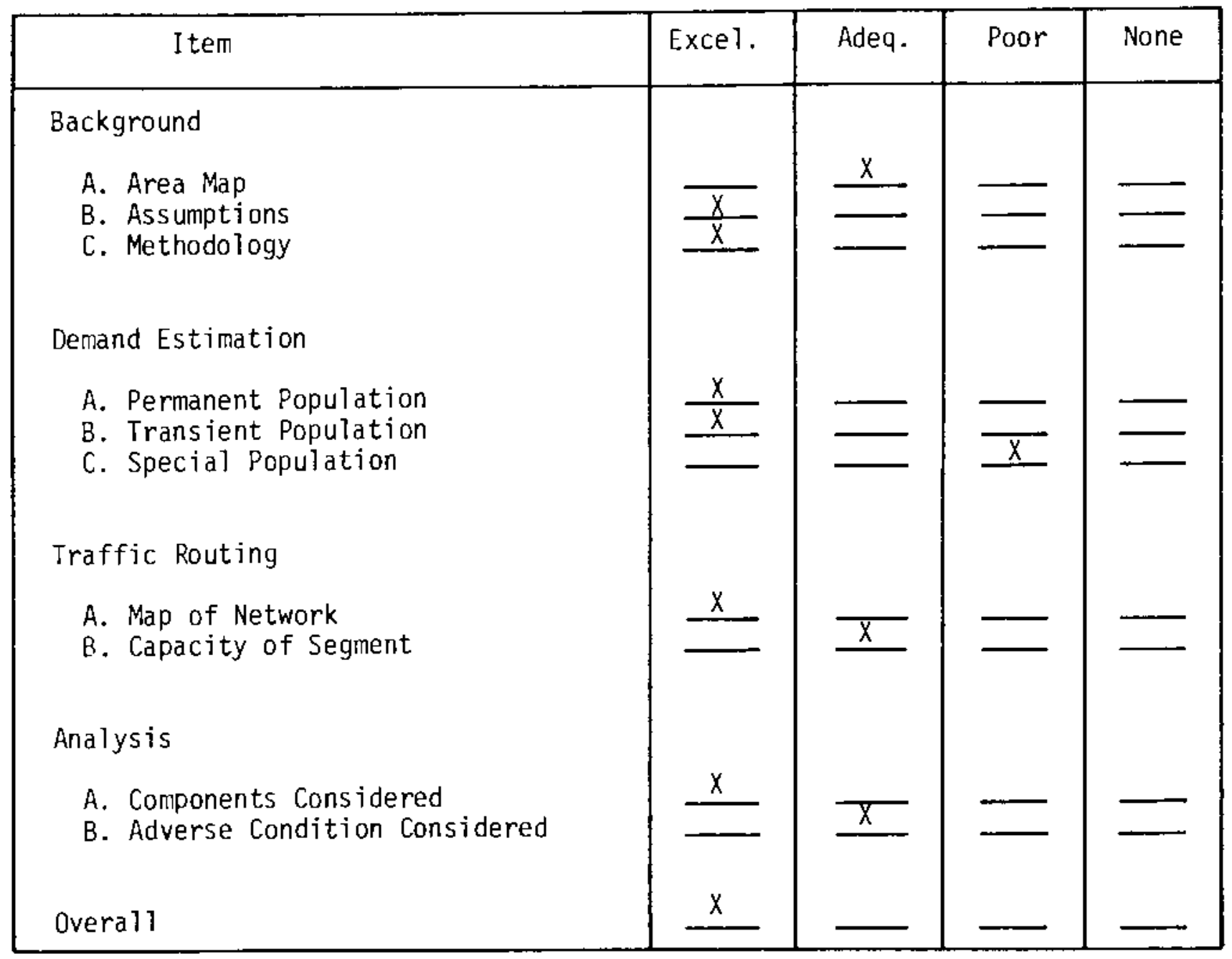

*Estimate prepared by FEMA contractor. 
EVALUATION OF EVACUATION TIME ESTIMATE

Millstone*

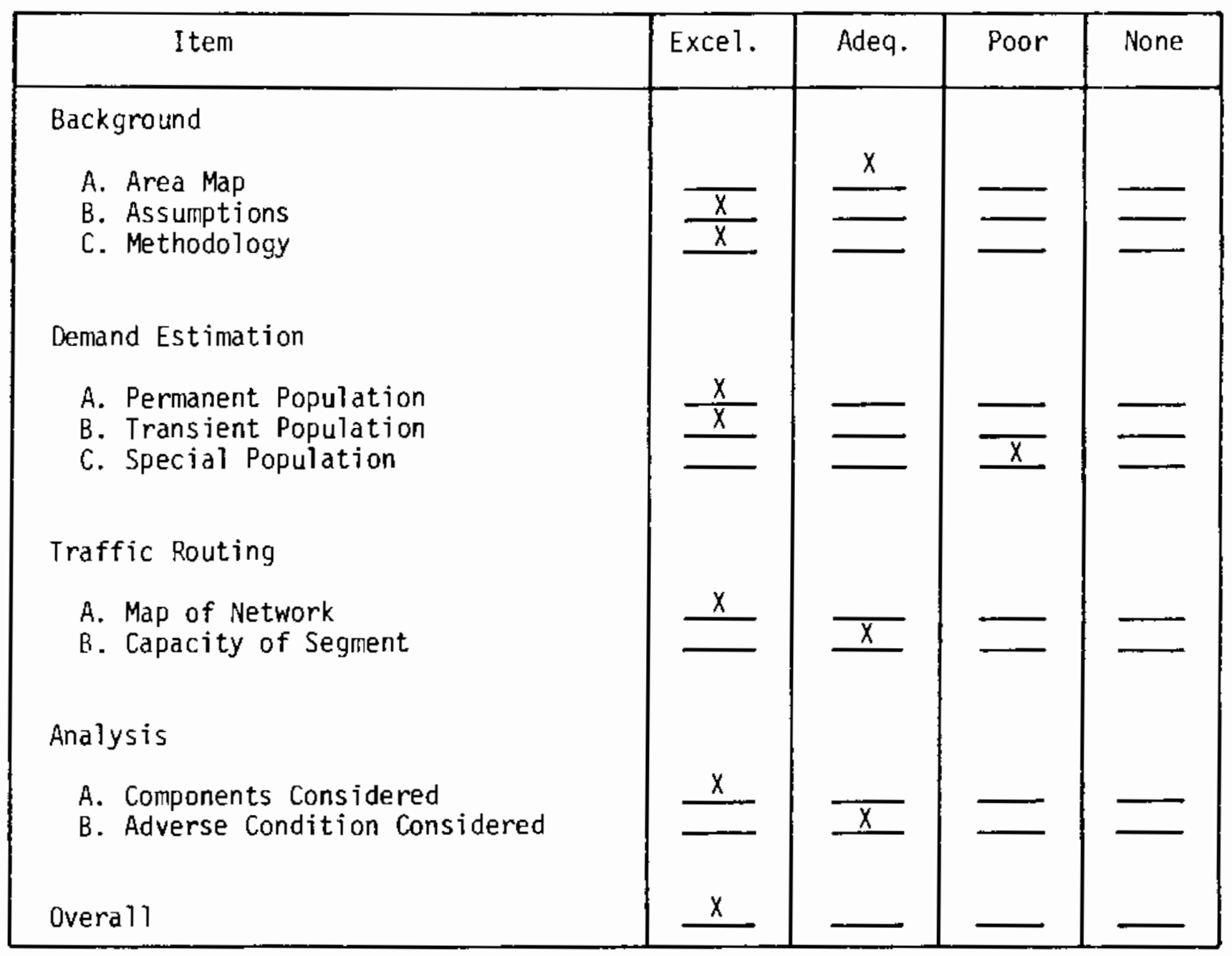

«Estimate prepared by FEMA contractor. 
EVALUATION OF EVACUATION TIME ESTIMATE

Three Mile Island*

\begin{tabular}{|c|c|c|c|c|}
\hline I tem: & Excel. & Adeq. & Poor & None \\
\hline $\begin{array}{l}\text { Background } \\
\text { A. Area Map } \\
\text { B. Assumptions } \\
\text { C. Methodology }\end{array}$ & $\begin{array}{l}x \\
x \\
\end{array}$ & $\underline{x}$ & & 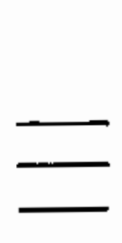 \\
\hline $\begin{array}{l}\text { Demand Estimation } \\
\text { A. Permanent Population } \\
\text { B. Transient Population } \\
\text { C. Special Population }\end{array}$ & $\underline{x}$ & $\underline{\square}$ & $\bar{x}$ & $\bar{x}$ \\
\hline $\begin{array}{l}\text { Traffic Routing } \\
\text { A. Map of Network } \\
\text { B. Capacity of Segment }\end{array}$ & $x$ & $X$ & & \\
\hline $\begin{array}{l}\text { Analysis } \\
\text { A. Components Considered } \\
\text { B. Adverse Condition Considered }\end{array}$ & $x$ & $x$ & & ( \\
\hline OveraIl & $x$ & . & & \\
\hline
\end{tabular}

*Estimate prepared by FEMA contractor. 


\begin{tabular}{|c|c|c|c|c|}
\hline Item & Exce 1. & Adeq. & Poor & None \\
\hline Background & & & & \\
\hline $\begin{array}{l}\text { A. Area Map } \\
\text { B. Assumptions } \\
\text { C. Methodology }\end{array}$ & $\bar{x}$ & $\frac{x}{x}$ & $\underline{z}$ & $\underline{\square}$ \\
\hline Demand Estimation & & & & \\
\hline $\begin{array}{l}\text { A. Permanent Population } \\
\text { B. Transient Population } \\
\text { C. Special Population }\end{array}$ & $\bar{x}$ & $\underline{x}$ & 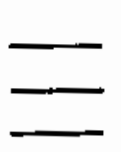 & $\underline{x}$ \\
\hline Traffic Routing & & & & \\
\hline $\begin{array}{l}\text { A. Map of Network } \\
\text { B. Capacity of Segment }\end{array}$ & - & $\frac{x}{x}$ & & $\square$ \\
\hline Analysis & & & & \\
\hline $\begin{array}{l}\text { A. Components Considered } \\
\text { B. Adverse Condition Considered }\end{array}$ & $x$ & $\bar{x}$ & $\longrightarrow$ & $\square$ \\
\hline Overall & _ & $x$ & - & - \\
\hline
\end{tabular}

*Estimate prepared by FEMA contractor. 


\section{APPENDIX E \\ SUMMARY OF EVACUATION TIME ESTIMATES \\ FOR FEMA ASSESSMENTS}

E-1 

EVACUATION TIME ESTIMATES

Beaver Valley*

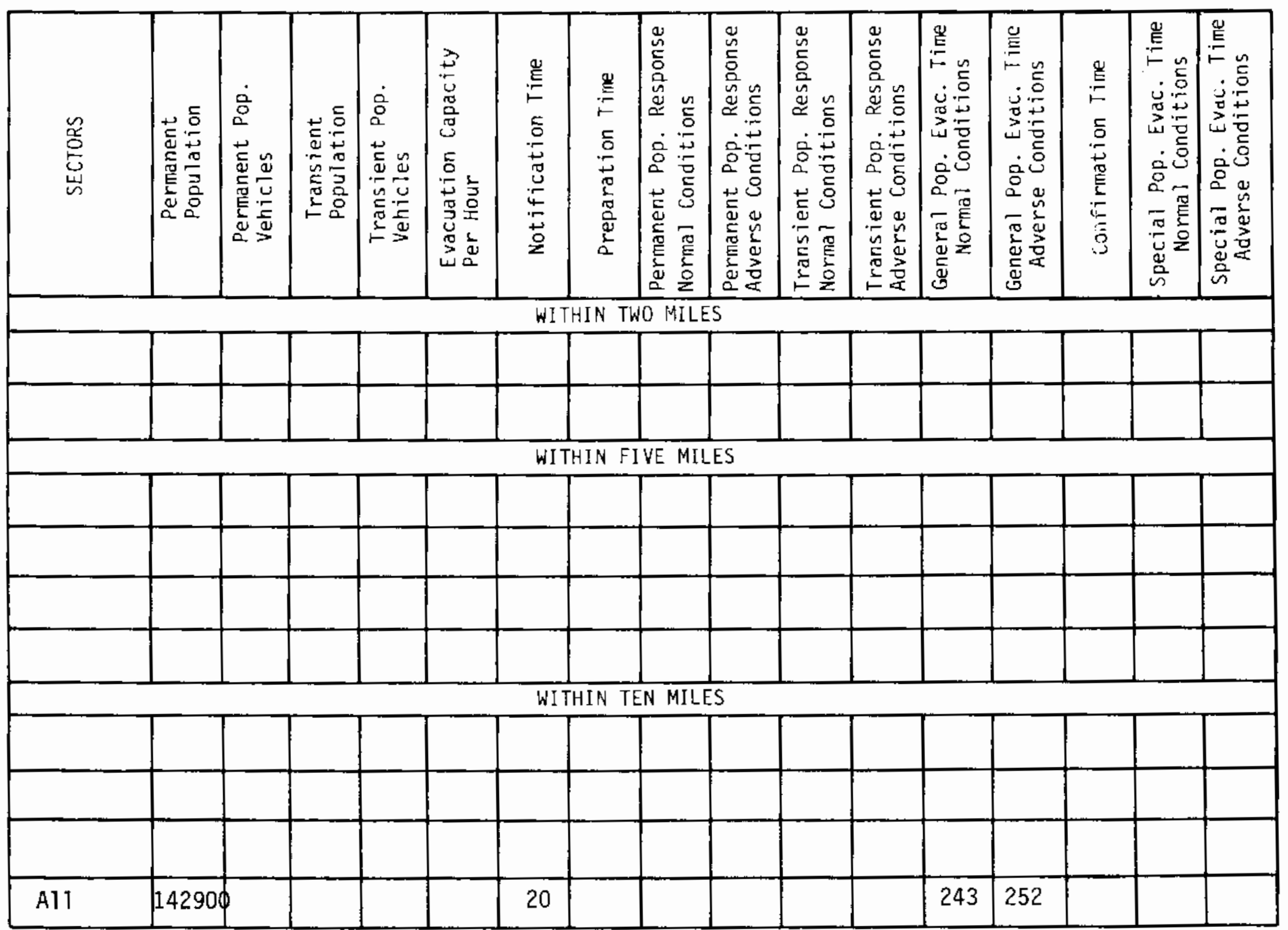

*Estimate prepared by FEMA contractor. 


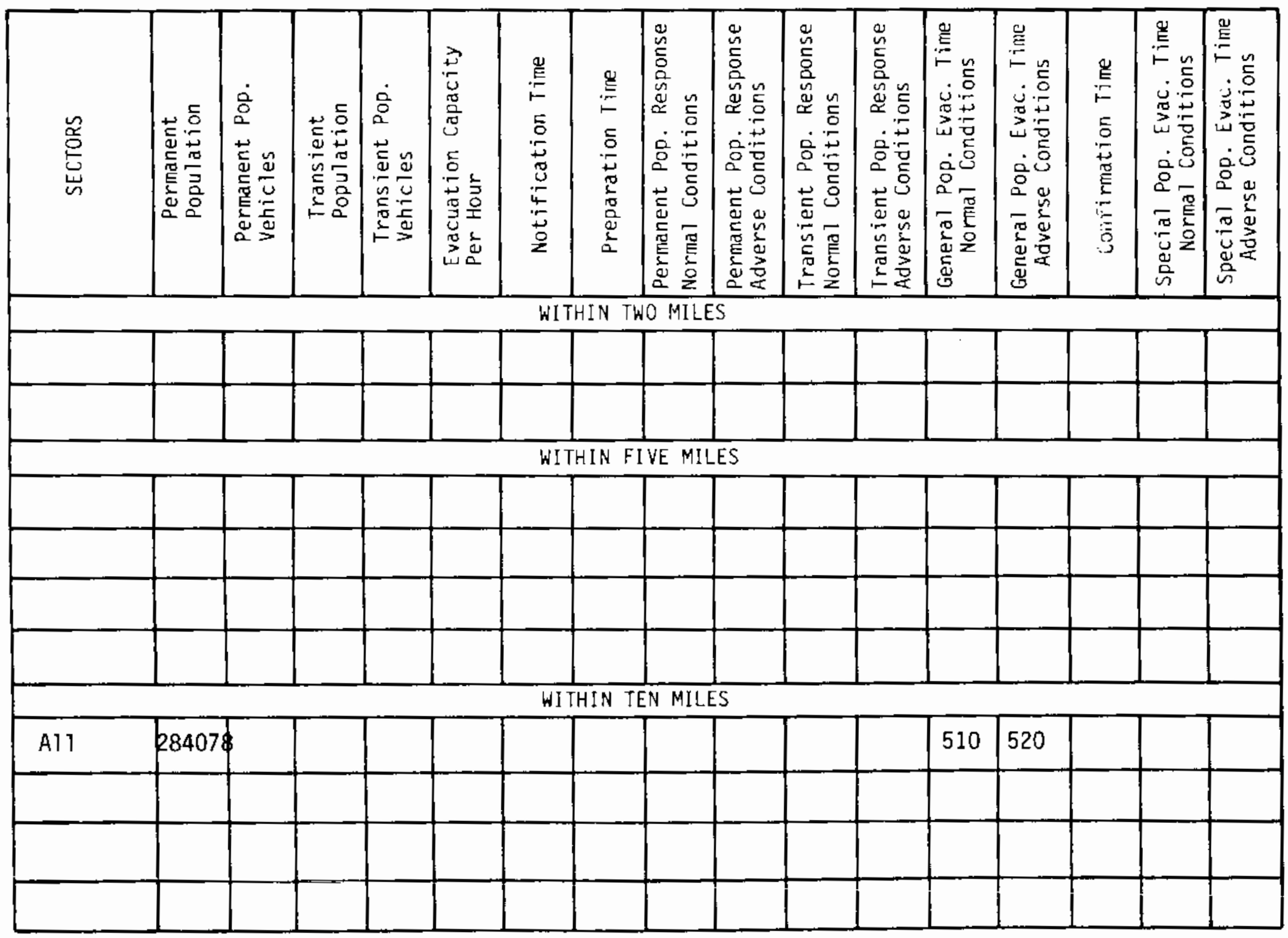

*Estimate prepared by FEMA contractor. 
EVACUATION TIME ESTIMATES

Maine Yankee*

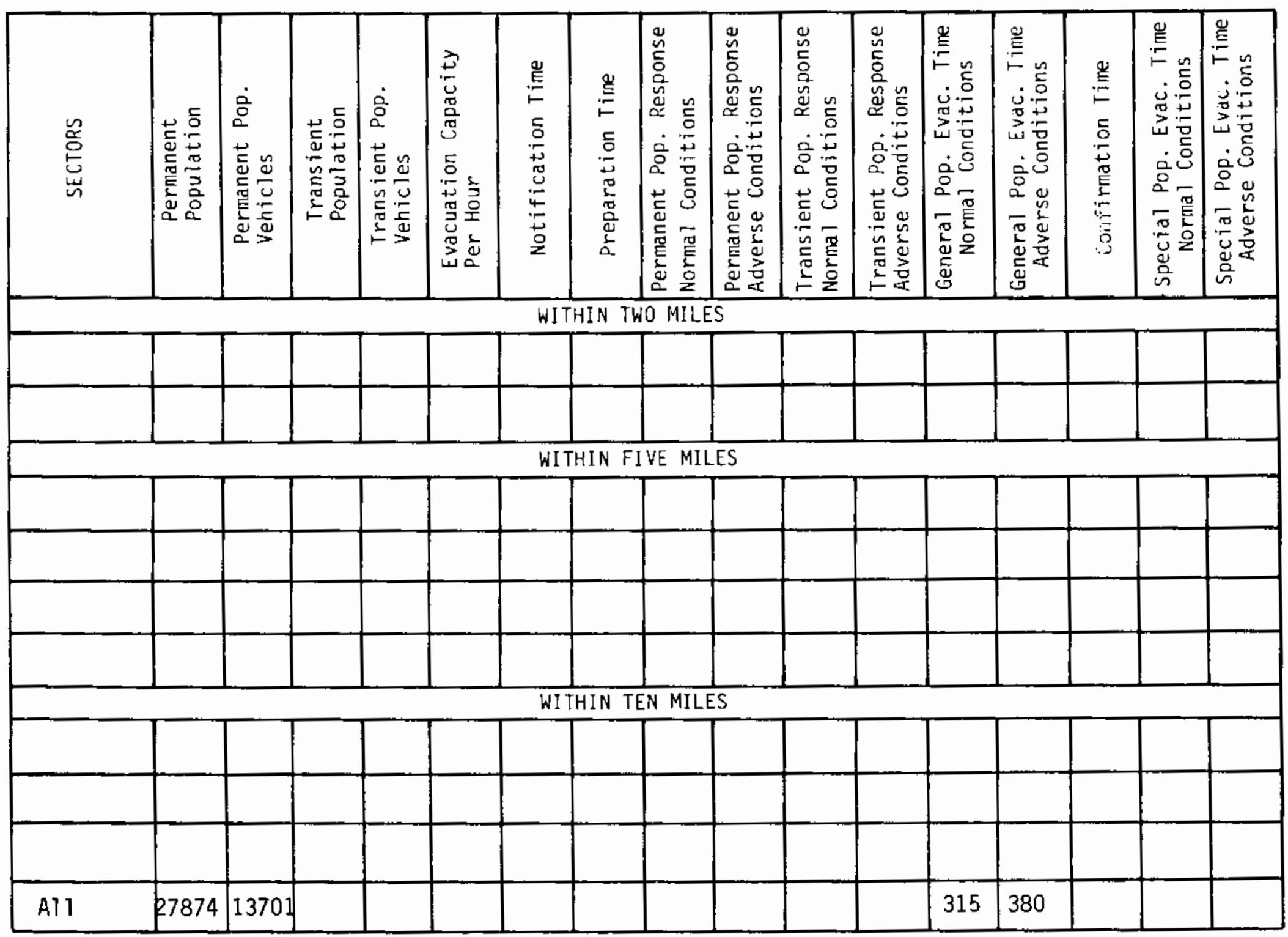

*Estimate prepared by FEMA contractor. 
EVACUATION TIME ESTIMATES

Millstone*

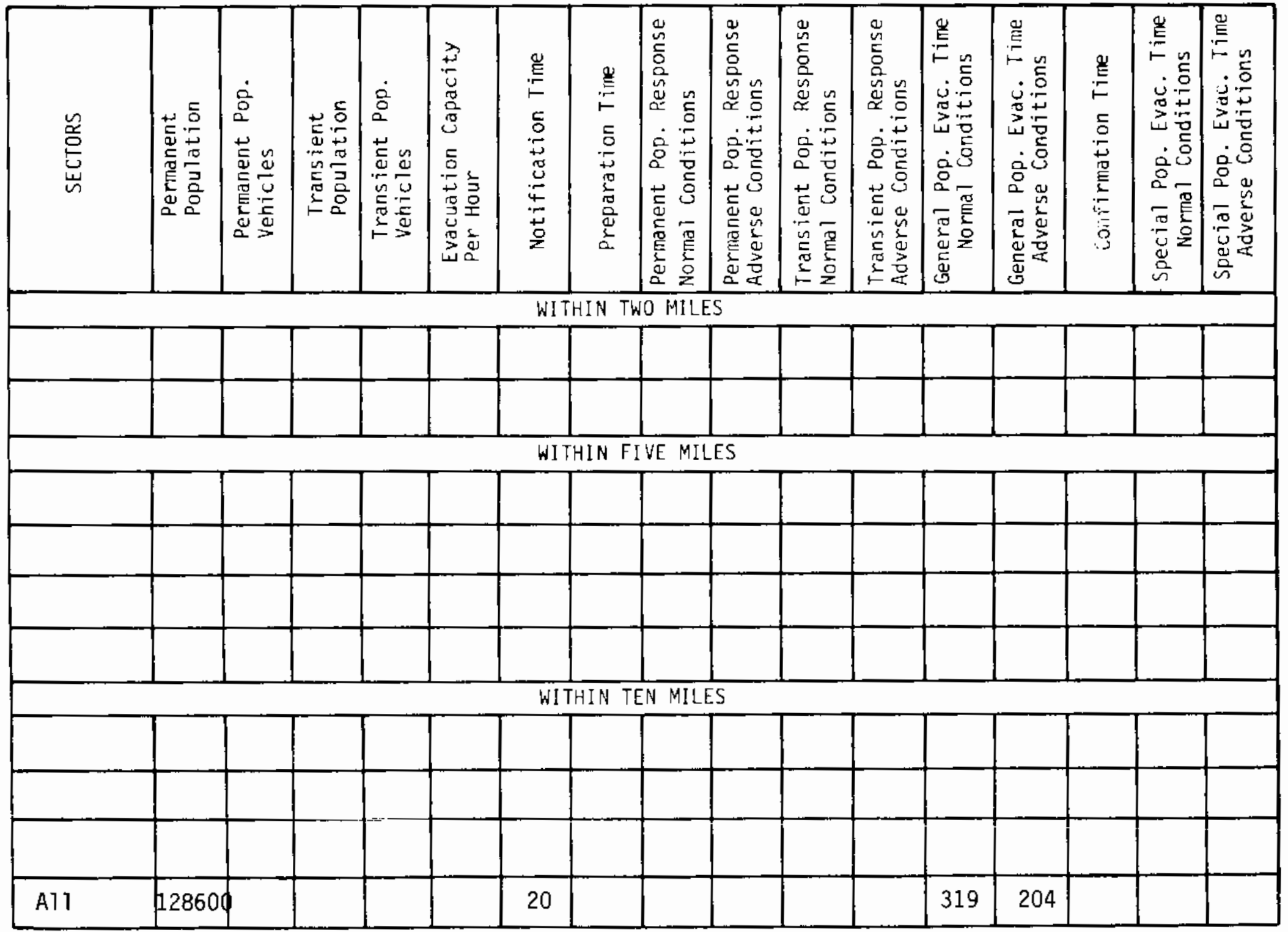

*Estimate prepared by FEMA contractor. 
EVACUATION TIME ESTIMATES

Three Mile Is Tand*

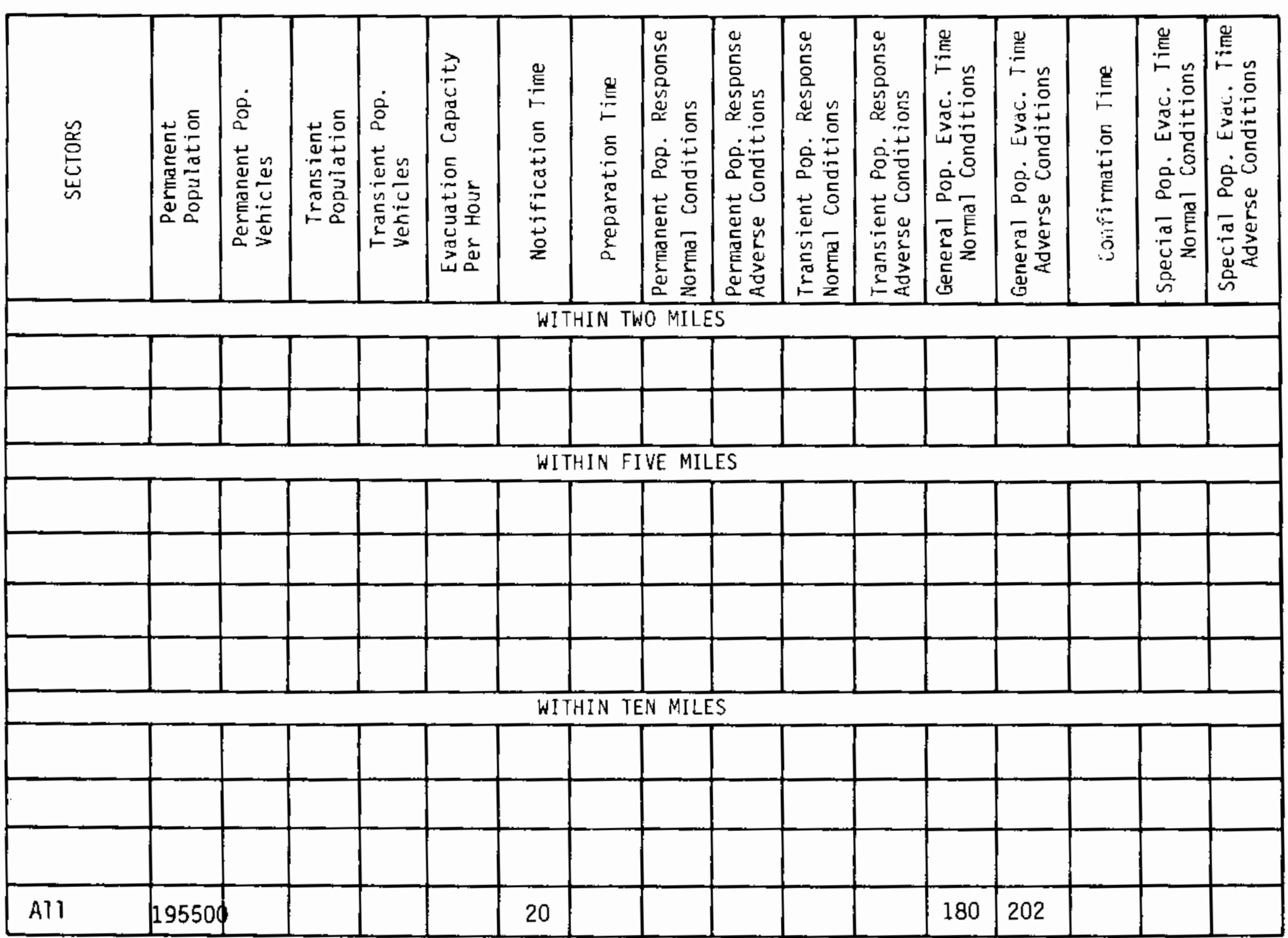

*Estimate prepared by FEMA contractor. 


\begin{tabular}{|c|c|c|c|c|c|c|c|c|c|c|c|c|c|c|c|c|}
\hline $\begin{array}{l}\text { 号 } \\
\text { 总 } \\
\text { 出 }\end{array}$ & 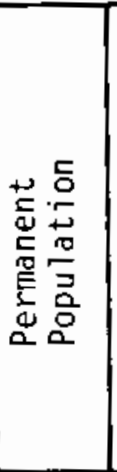 & 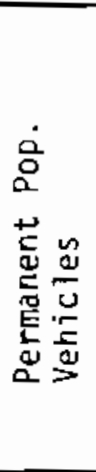 & 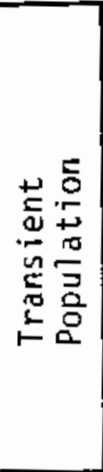 & 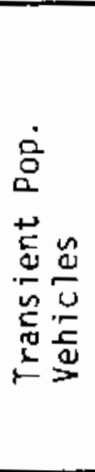 & 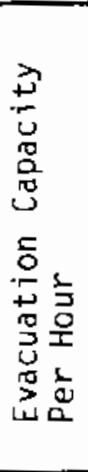 & 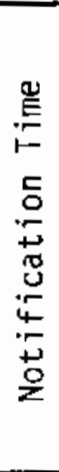 & 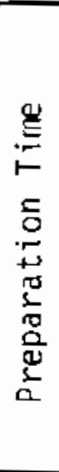 & 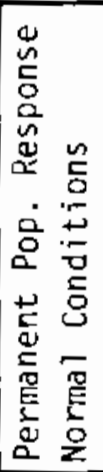 & 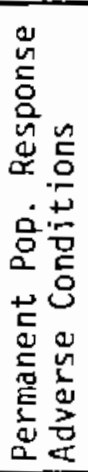 & 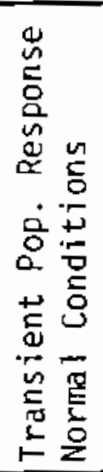 & 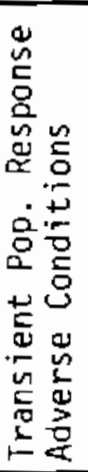 & 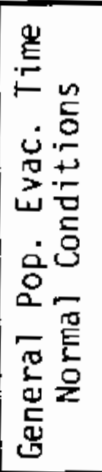 & 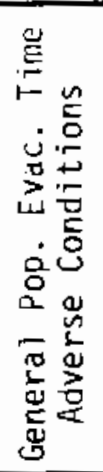 & 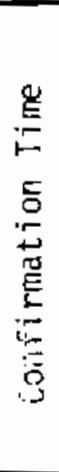 & 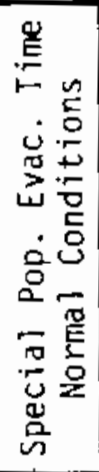 & 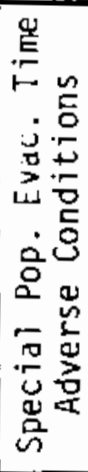 \\
\hline \multicolumn{17}{|c|}{ WITHIN TWO MILES } \\
\hline A11 & & & & & & & & & & & & 220 & & & & \\
\hline \multicolumn{17}{|c|}{ WITHIN FIVE MILES } \\
\hline A]1 & & & & & & & & & & & & 220 & & & & \\
\hline & & & & & & & & & & & & & & & & \\
\hline & & & & & & & & & & & & & & & & \\
\hline & & & & & & & & & & & & & & & & \\
\hline \multicolumn{17}{|c|}{ WITHIN TEN MILES } \\
\hline A11 & 240000 & & & & & & & & & & & 285 & 350 & & & \\
\hline & & & & & & & & & & & & & & & & \\
\hline & & & & & & & & & & & & & & & & \\
\hline & & & & & & & & & & & & & & & & \\
\hline
\end{tabular}

*Estimate prepared by FEMA contractor. 


\section{OISTRIBUTION}

OFFSITE

(1) A. A. Churm

D.0.E. Patent Division

98005 Cass Avenue

Aryonne, I11. 60439

(300) U.S. Nuclear Regulatory Commission

Division of Technical Information and Document Control

7920 Norfolk Avenue

Bethesda, MD. 20014

(2) D.0.E. Technical Information Center

(10) S. L. Ramos, Chief Emergency Preparedness Development Branch

U.S. Nuclear Regulatory Comission

7920 Norfolk Avenue

Bethesda, MD. 20014

ONSITE

(30) Pacific Northwest Laboratory

W. A. Glass (1)

3. M. Selby (1)

T. H. Essig (1)

A. E. Desrosier (20)

Publishing Coordination (2)

Technical Infornation (5) 



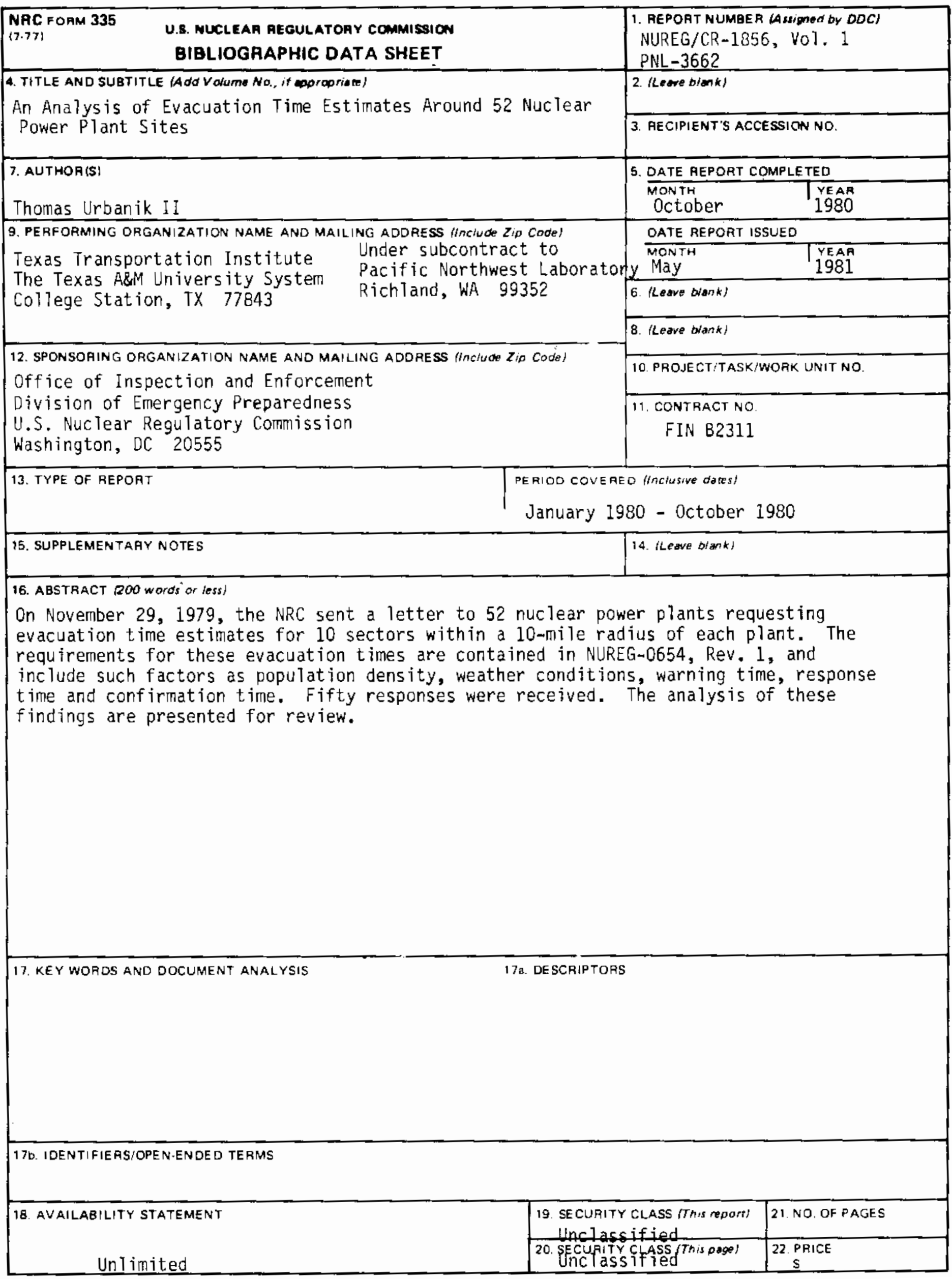


